\title{
Vibrotactile Feedback for Human Balance Improvement: Experimental Investigation of Optimal Feedback Location
}

\author{
by \\ Christiane Courtemanche, BSC(Eng) \\ A thesis submitted to the \\ Faculty of Graduate and Postdoctoral Affairs \\ in partial fulfillment of the requirements for the degree of
}

Master of Applied Science in Mechanical Engineering
Ottawa-Carleton Institute for Mechanical and Aerospace and Computer Engineering Department of Mechanical and Aerospace Engineering Carleton University Ottawa, Ontario January, 2017
(C)Copyright

Christiane Courtemanche, 2017 
The undersigned hereby recommends to the

Faculty of Graduate and Postdoctoral Affairs acceptance of the thesis

\section{Vibrotactile Feedback for Human Balance Improvement: Experimental Investigation of Optimal Feedback Location}

submitted by Christiane Courtemanche, BSC(Eng)

in partial fulfillment of the requirements for the degree of

Master of Applied Science in Mechanical Engineering

R. Miller, Department Chair

Ottawa-Carleton Institute for Mechanical and Aerospace and Computer Engineering Department of Mechanical and Aerospace Engineering

Carleton University

January, 2017 


\section{Abstract}

This thesis characterizes the use of vibrotactile feedback and evaluates the effect of feedback location on human balance via three main experiments. The first experiment characterizes common tactor types and compares their performance based on participants' perception. The better performing tactor is used in the second experiment, which investigates the optimal body location for feedback through participants' performance in sensing tactor array feedback. This study compares neck, waist, wrist, and ankle feedback weighted scores of reaction time, ability to detect feedback, and ability to discern the activated tactor and its vibration intensity. The best-performing locations, wrist and ankle, are used in the third study to show vibrotactile feedbacks positive effect on participants' balance and to identify the optimal body location for

feedback. Feedback, independent of location, significantly improved percentage time spent in the deadzone, and in some tests also significantly improved AP and ML trunk tilt. 


\section{Acknowledgments}

First and foremost, I would like to thank my thesis supervisor, Professor Mojtaba Ahmadi, for his overwhelming support and for always taking the time to discuss matters at hand without making meetings feel rushed, even though he was incredibly busy. But mostly, I want to thank him for enabling me to further my knowledge more than I would have thought possible in two years. I would also like to thank my co-supervisor, Dr. Allen Huang, for being so invested in this project that he drove to Carleton for 8 am meetings and took the time to read my thesis, even while traveling. I feel very fortunate to have had the chance to work with him and learn more about the physician's point of view on assistive devices.

I am also very appreciative of my colleagues in the ABL lab and the good discussions we had; some of those conversations often turned a bad day into a great one. A special thanks to Stephanie Eng for her involvement in the first phase of this work and to Omar Masaud for teaching me most of what I know about circuit design.

Finally, my deep and sincere gratitude to my family for their continuous love, help and support. I am grateful to my sister for always being there for me and believing in me. I am forever indebted to my parents for giving me the opportunities and experiences that have made me who I am. This journey would not have been possible without them, and I dedicate this milestone to them. Lastly, I am truly grateful for the undying love and support of my fiance who pushes me everyday to be the best version of myself and who was always the best influence when I needed to work. 


\section{Table of Contents}

Abstract $\quad$ iii

Acknowledgments $\quad$ iv

Table of Contents $\quad$ v

List of Tables $\quad \mathrm{x}$

List of Figures $\quad$ xiii

Nomenclature $\quad$ xix

1 Introduction 1

1.1 Motivation ......................... 1

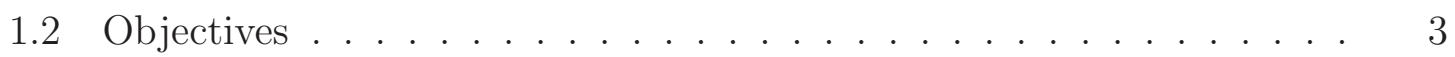

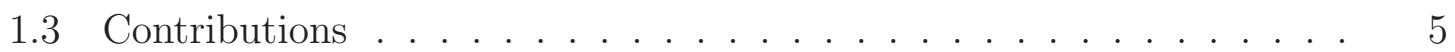

1.4 Overview ............................... 6

2 Literature Review $\quad 8$

2.1 Background ........................... 8

2.2 Methods .............................. 9

2.2.1 Types of Studies . . . . . . . . . . . . . . 9

2.2.2 Types of Participants . . . . . . . . . . . . . . . . . . . 10

2.2.3 Types of Interventions . . . . . . . . . . . . . . . . . . . . . . 10 
2.2.4 Types of Outcome Measures . . . . . . . . . . . . . . . 11

2.2 .5 Meta-Analysis . . . . . . . . . . . . . . . . . 13

2.3 Results . . . . . . . . . . . . . . . . . . . . 15

2.3 .1 Summary of Studies . . . . . . . . . . . . . . . 15

2.3.2 Meta-Analysis of Results . . . . . . . . . . . . . . . . 18

2.3.3 Comparison of Non-Meta-Analyzed Results . . . . . . . . . . 22

2.4 Discussion . . . . . . . . . . . . . . . . . . . . . . . 30

2.4 .1 Meta-Analysis . . . . . . . . . . . . . . 30

2.4 .2 Age effects. . . . . . . . . . . . . . . 30

2.4 .3 Secondary task effects $\ldots \ldots \ldots \ldots$

2.4.4 Balance impairment effects . . . . . . . . . . . . . . . . 32

2.4 .5 Naivety effects. . . . . . . . . . . . . . . . . 32

2.4 .6 System Optimization . . . . . . . . . . . . . . . . 33

2.4.7 Functional Bandwidth and Stimulus Properties . . . . . . 35

2.4 .8 Future Directions . . . . . . . . . . . . . . . . . . 36

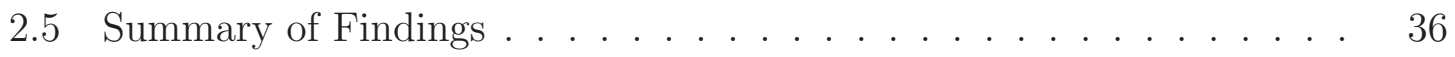

3 Tactor Vibration Characterization $\quad 39$

3.1 Objective . . . . . . . . . . . . . . . . . . . . 39

3.2 Vibration Sensing . . . . . . . . . . . . . . . . . . 40

3.3 Methods . . . . . . . . . . . . . . . . . . . 43

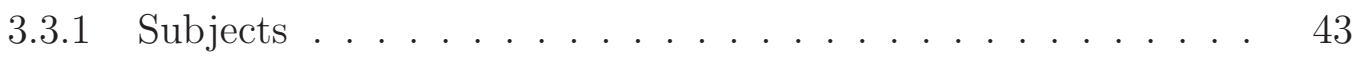

3.3 .2 Procedure . . . . . . . . . . . . . . . . . . . . 43

$3.3 .3 \quad$ Experimental Setup . . . . . . . . . . . . . . . . . . . 44

3.3.4 Measures of Comparison . . . . . . . . . . . . . . 54

3.3.5 Data Analysis . . . . . . . . . . . . . . . . . . 54

3.4 Results and Discussion . . . . . . . . . . . . . 56

3.4 .1 Data Filtering . . . . . . . . . . . . . . . 56 
3.4.2 Acceleration and Displacement Amplitude Calculation . . . . 61

3.4.3 Vibration Characteristics . . . . . . . . . . . . 62

3.4.4 Tactor Selection for Future Studies . . . . . . . . . . . . . . . 69

3.4.5 Vibration Detection Prediction . . . . . . . . . . . 71

3.5 Summary . . . . . . . . . . . . . . . . . . 73

4 Body Location Optimization for Vibrotactile Feedback $\quad 75$

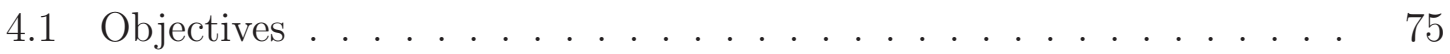

4.2 Methods . . . . . . . . . . . . . . . . . 77

4.2.1 Subjects .......................... 77

4.2.2 Procedure . . . . . . . . . . . . . . . . . 77

4.2.3 Instrumentation . . . . . . . . . . . . . . . . . . . . 80

4.2.4 Arduino Programming . . . . . . . . . . . . . . . . 85

4.2.5 Data Analysis . . . . . . . . . . . . . . 87

4.3 Results and Discussion . . . . . . . . . . . . . . . . . . . . . 92

4.3.1 Identifying Best Body Location for Feedback . . . . . . . . . . 92

4.3.2 BMI Effect on Ability to Sense Feedback . . . . . . . . . . . . 97

4.3.3 Ability to predict if feedback will be detected . . . . . . . . . 99

4.4 Summary . . . . . . . . . . . . . . . . . . . . 102

5 Vibrotactile Feedback Location Effects on Balance 104

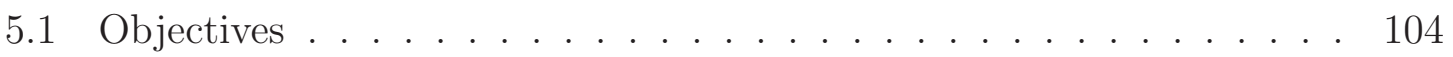

5.2 Methods . . . . . . . . . . . . . . . . . 105

5.2.1 Subjects ......................... 105

5.2.2 Procedure . . . . . . . . . . . . . . . . . . . . . 105

5.2 .3 Experimental Set-Up . . . . . . . . . . . . . . . . 107

5.2 .4 Data Analysis . . . . . . . . . . . . . . 117

5.3 Results and Discussion . . . . . . . . . . . . . . . . . . . 118 
5.3.1 Swaypath Variation with Feedback . . . . . . . . . . . 118

5.3.2 RMS AP and ML trunk tilt . . . . . . . . . . . . . 120

5.3 .3 Dominant Movement Frequency . . . . . . . . . . . . . . . 124

5.3 .4 Reaction Time . . . . . . . . . . . . . . . . . . . . 125

5.3.5 Percentage Time Spent in Deadzone . . . . . . . . . . . 128

5.4 Summary . . . . . . . . . . . . . . . . . . . . 130

6 Conclusions 132

6.1 Type of Tactor for Use in Future Studies . . . . . . . . . . . . 133

6.2 Feedback Detection Predictor . . . . . . . . . . . . . . 133

6.3 Optimal Location for Vibrotactile Feedback . . . . . . . . . . . 134

6.4 Vibrotactile Feedback Effect On Balance . . . . . . . . . . . . . 135

6.5 Future Work . . . . . . . . . . . . . . . . . . . . . . . 135

6.5.1 Improve Balance Measures . . . . . . . . . . . . . . . . . 135

6.5.2 Build Integrated Balance Feedback System . . . . . . . . . 136

6.5.3 Older Adult and Patient Testing _. . . . . . . . . . 137

6.5.4 Improve Testing Protocol . . . . . . . . . . . . . . . . 137

6.5.5 Study Other Representations of Balance . . . . . . . . . 138

$\begin{array}{ll}\text { Appendices } & 139\end{array}$

A Meta-analysis results for the overall biofeedback effect on AP tilt (regardless of tactor/sensor location) . . . . . . . . . . . . . 139

B Meta-analysis results for the overall biofeedback effect on ML tilt (regardless of tactor/sensor location) _ . . . . . . . . . 140

C Minimum, Mean and Maximum Values for the ERM and LRA Measures141

D Schematic Representation of the Test Circuit Connections for the Location Optimization study . . . . . . . . . . . . . . . . 148

E Arduino Code for Location Optimization Testing . . . . . . . . 150 
F Linear Regression of Evaluation Criteria to BMI Results . . . . . . 163

G Linear Regression of Acceleration and Displacement Amplitude to BMI Results . . . . . . . . . . . . . . . . . . . . . . 164

H Arduino Code for Balance Effects Testing . . . . . . . . . . 166

I Swaypaths for Individual Participants . . . . . . . . . . . . . . 181

J Significance of Difference Between Feedback Type Performance on Evaluation Criteria . . . . . . . . . . . . . . . . . . . . . 194

K Means, Standard Deviations and P values for Each of the Tests . . . 197

List of References 


\section{List of Tables}

2.1 Davis 2010 Study Design . . . . . . . . . . . . . . . 16

2.2 Goodworth 2011 Study Design . . . . . . . . . . . . . . . . 16

2.3 Haggerty 2012 Study Design . . . . . . . . . . . . . . . . 17

2.4 Janssen 2010 Study Design . . . . . . . . . . . . . . . . . . . 17

2.5 Lee 2013 Study Design . . . . . . . . . . . . . . . . . . . . . . 18

2.6 Peterka 2006 Study Design . . . . . . . . . . . . . . . . . . 18

2.7 Sienko 2008 Study Design _. . . . . . . . . . . . . . . . . . . 19

2.8 Sienko 2010 Study Design _ . . . . . . . . . . . . . . . . . . 19

2.9 Tino 2011 Study Design . . . . . . . . . . . . . . . 20

2.10 Verhoeff 2009 Study Design . . . . . . . . . . . . . . . . . 20

2.11 Wall 2001 Study Design . . . . . . . . . . . . . . . . 21

2.12 Wall 2005 Study Design . . . . . . . . . . . . . . . . . 21

2.13 Wall 2009 Study Design _. . . . . . . . . . . . . . . 22

2.14 Summary of Review Findings . . . . . . . . . . . . . . . . . 38

3.1 Types of stimulus sensed by each type of mechanoreceptors . . . . . . 41

3.2 Participant Demographics . . . . . . . . . . . . . . . . . . 43

3.3 Arduino settings for each tested voltage and corresponding voltages across the ERM and LRA tactors . . . . . . . . . . . . . . . 51

3.4 Percentage times feedback was felt for each tactor type at each voltage setting ............................. 70

4.1 Participant Demographics . . . . . . . . . . . . . . . 77 
4.2 Meaning of Arduino Program's Location Codes . . . . . . . . . . . . 86

4.3 Evaluation criteria's scores and weights used in total score (TS) calcu-

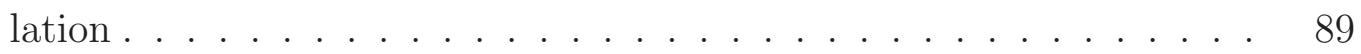

4.4 Practicality scores for each location . . . . . . . . . . . . . . . 90

4.5 Total score excluding practicality $(T S)$ and total score including practicality $T S_{P}$ for each body location . . . . . . . . . . . . . . . 93

4.6 Mean(SD) reaction time and percentage detected feedback, correctly identified tactor and correctly identified intensity . . . . . . . . . . 94

4.7 Significance of differences between measures for each body location (Neck, Wrist, Waist and Ankle) when Greenhouse-Geisser correction is applied . . . . . . . . . . . . . . . . . . . . 95

4.8 p values comparing body location means for each evaluation criterion 95

5.1 Experimental Trials . . . . . . . . . . . . . . . . 107

5.2 Participant Groups . . . . . . . . . . . . . . . . . . . 107

F.1 Correlation coefficients ( $\mathrm{p}$ values) obtained from the linear regression analysis between each evaluation criterion and BMI . . . . . . . . 163

G.1 Parameters related to the linear regression between various acceleration amplitude data and the corresponding participant BMI . . . . . . . . 164

G.2 Parameters related to the linear regression between various displacement amplitude data and the corresponding participant BMI . . . . . 165

J.1 Significance of ML tilt differences found using the Repeated Measures

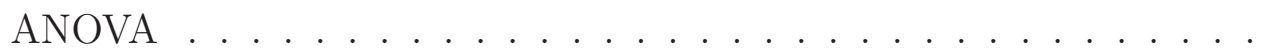

J.2 Significance of AP tilt differences found using the Repeated Measures

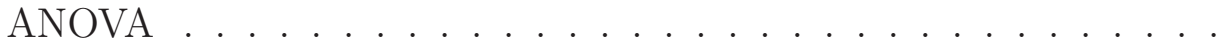

J.3 Significance of reaction time differences found using the Repeated Measures ANOVA . . . . . . . . . . . . . . . . . . . 195 
J.4 Significance of percentage time spent in the deadzone differences found using the Repeated Measures ANOVA _. . . . . . . . . . . 195

J.5 Significance of dominant motion frequency differences found using the Repeated Measures ANOVA . . . . . . . . . . . . . . 196

K.1 RMS AP tilt. . . . . . . . . . . . . . . . . . . . . 197

K.2 RMS ML tilt . . . . . . . . . . . . . . . . . . . . . . 198

K.3 Dominant Movement Frequency . . . . . . . . . . . . . . . . . 199

K.4 Reaction Time . . . . . . . . . . . . . . . . . . . . . . . . . . 199

K.5 Percent Time in the Deadzone . . . . . . . . . . . . . . . 200 


\section{List of Figures}

3.1 Depiction of mechnoreceptor shape, size and location within the skin [25]. 40

3.2 Pacinian corpuscle's minimum vibration displacement for detection as a function of vibration frequency, taken from [28]. . . . . . . . . . . . 42

3.3 Circuit and its components used in this study : A) Arduino Mega 2560 board, B) TexasInstruments DRV 2603 chip, C) tactor powering wires, D) tactor and accelerometer assembly, E) accelerometer communica-

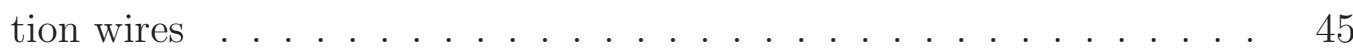

3.4 Part breakdown of the ERM actuator as shown on the Precision Microdrives web page ..................... 46

3.5 310-003 tactor datasheet performance. . . . . . . . . . . . . . . . 46

3.6 LRA vibration amplitude as a function of the $\mathrm{AC}$ voltage frequency powering it $[33] \ldots \ldots \ldots \ldots$. . . . . . . . . . . . . . . . . . . . . 47

3.7 Part breakdown of the LRA actuator Precision Microdrives web page 48

3.8 C10-000 tactor datasheet performance. . . . . . . . . . . . . 48

3.9 A dynamometer is used to ensure constant bandage pressures when attaching tactor/accelerometer setup to participants' bodies. . . . . . 52

3.10 Body locations tested as part of the study, with biomaterial type indi-

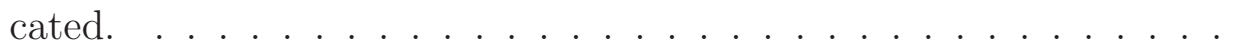

3.11 Meaning of true positive, false positive, true negative, and false negative. 55

3.12 Effects of filtering the acceleration signal when the circuit is powered but no voltage is applied across the tactor. . . . . . . . . . . 58 
3.13 Plots for PSD vs. frequency where (a) is the unfiltered ERM signal's PSD, (b) is the unfiltered LRA signal's PSD, (c) is the filtered ERM signal's PSD and (d) is the filtered LRA signal's PSD for trials where the mounting point of the tactor was moved repeatedly. . . . . . . . .

3.14 Plots of maximum PSD for vibration in the $\mathrm{x}, \mathrm{y}$ and $\mathrm{z}$ directions for each tactor type on each biomaterial tested. . . . . . . . . . . .

3.15 Plots of tactor vibration frequency in the $\mathrm{x}, \mathrm{y}$ and $\mathrm{z}$ directions for each tactor type on each biomaterial tested. . . . . . . . . . . . . .

3.16 Plots of acceleration amplitude in the $\mathrm{x}, \mathrm{y}$ and $\mathrm{z}$ directions for each tactor type on each biomaterial tested. . . . . . . . . . . . .

3.17 Experimental set-up used by Precision Microdrives to determine vibration frequency, acceleration amplitude and efficiency as a function of voltage. . . . . . . . . . . . . . . . . .

3.18 Plots of tactor vibration displacement amplitude in the $\mathrm{x}, \mathrm{y}$ and $\mathrm{z}$ directions for each tactor type on each type of biomaterial tested. . . 68

3.19 Specificity and sensitivity associated with each metric for the LRA. . 71

4.1 The tactor placement for each testing location was as shown above for the (a) neck (b) non-dominant wrist (c) waist and (d) left ankle. . . . 79

4.2 This was the circuit used to conduct this study. The accelerometers/tactors (A), tactor powering circuit (B), Arduino (C) and user interface (D) are depicted. Ribbons 1, 2, 3 and 4 are used to link the accelerometers and tactors to the breadboard. Ribbon 5 provides the PWM input for the tactor powering circuits. Ribbon 6 allows communication between the Arduino and the accelerometers. Ribbon 7 allow the Arduino to read button-press instances from the user interface. This circuit's schematic representation can be found in Appendix D. . . . . 
4.3 This is the circuit used to power one ERM using a PWM signal filtered by the first and second low pass filters and amplified by the MAX4019 buffer. The pin numbers on the operational amplifier correspond to the pin numbers for the first operational amplifier on the SOIC chip for the MAX4019. For the other two operational amplifiers on the chip, the corresponding pin connections are interchangeable as follows: $5 / 10 / 12,6 / 9 / 13$ and $7 / 8 / 14$, all other pin connections to the ground of power source only need to be made once per MAX4019 chip. The resistor and capacitor values for the circuit are: $\mathrm{R} 1=15 \mathrm{k} \Omega, \mathrm{R} 2=33$

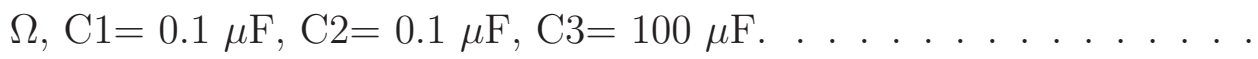

4.4 The total scores for each body location are given as a function of the criteria weight, each inset identifying the effect of one criterion's weight on each location's total score given that all other criteria weights are

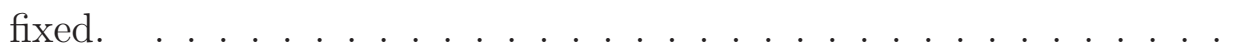

4.5 The above plots depict the relationships between the performance on each evaluation criterion of each subject and the respective subject's

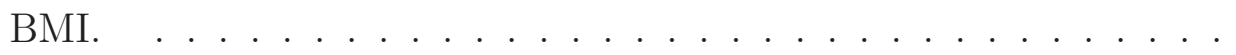

4.6 The variations in sensitivity, specificity and their sum is shown as a function of the cutoff value used to identify a vibration event as being detected or not by a participant for both the (a) acceleration and (b) displacement amplitudes used as predictors. . . . . . . . . . . .

5.1 Experimental setup. The trunk tilt is sensed using the inertial motion unit (A) and recorded by the Arduino (B) for later analysis. The Arduino then identifies if feedback should be provided and sends the appropriate signal to the tactor driving circuits (C) who activate either the tactors attached to the waist (D) or to the ankle (E) depending on the feedback condition (no, ankle or waist feedback). 
5.2 This figure demonstrates how each of the experimental circuit's components are worn by participants in this study through the use of a (a) front view, (b) side view and (c) back view. . . . . . . . . . . . . 109

5.3 Orientation of the MPU6050 measurement axes with respect to participant trunk tilt during experiments. The positive acceleration axes are shown in black and the positive angular acceleration directions are shown in grey. . . . . . . . . . . . . . . . . . . . . . . 110

5.4 Block diagram of filter system used to fuse accelerometer and gyroscope estimates of the tilt angle $\theta \ldots \ldots \ldots \ldots$

5.5 These are the feedback zones used in the experiment, the Deadzone (balanced zone) corresponds to tilt angles in the ML and AP direction of 0 to 1 degree, Zone 2 (slightly unbalanced zone) corresponds to tilt angles ranging from 1 to 3 degrees and Zone 3 (very unbalanced zone) corresponds to tilt angles greater than 3 degrees. . . . . . . . . . . . . 116

5.6 Sample swaypaths showing (a) either poor understanding or ineffective training for ankle feedback on double foam with eyes closed and (b) lack of sensitivity to feedback on the lower back for, left to right, standing eyes closed on a hard surface, eyes open on single-foam and eyes closed on single-foam. . . . . . . . . . . . . . . . . . . . 119

5.7 These are the mean values for the RMS AP and ML tilt for each Trial and each feedback condition. Feedback conditions' differences are indicated as not significant (n.s.) or significant with $\mathrm{p}<0.05\left(^{*}\right)$. The mean, SD and $\mathrm{p}$ values are all reported in Appendix K, Tables K.1 and K.2. . . . . . . . . . . . . . . . . . . . . . . . . . . . . . . 123 
5.8 These are the mean values and the associated error bars for the dominant movement frequency for each Trial with no feedback or feedback on the ankle or waist. Feedback conditions' differences are indicated as not significant (n.s.) or significant with $\mathrm{p}<0.05(*) \ldots . . . . .$.

5.9 These are the mean values and the associated error bars for the reaction time for each Trial with no feedback or feedback on the ankle or waist. Feedback conditions' differences are indicated as not significant (n.s.) or significant with $\mathrm{p}<0.05(*)$. The mean, $\mathrm{SD}$ and $\mathrm{p}$ values are all reported in Appendix K, Table K.4. . . . . . . . . . . . . . . . .

5.10 These are the mean values and the associated error bars for the percentage time spent in the deadzone (balanced) for each Trial with no feedback or feedback on the ankle or waist. Feedback conditions' differences are indicated as not significant (n.s.) or significant with $\mathrm{p}<0.05$ (*). The mean, SD and p values are all reported in Appendix K, Table

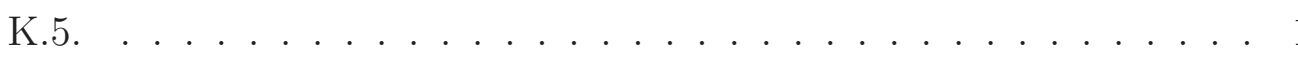

D.1 This shows the schematic connections for each of the ribbons shown in Figure $4.2 \ldots \ldots \ldots \ldots$

I.1 Participant 1's swaypath for all tests performed. . . . . . . . . . . 182

I.2 Participant 2's swaypath for all tests performed. . . . . . . . . . . 183

I.3 Participant 3's swaypath for all tests performed. . . . . . . . . . . . 184

I.4 Participant 4's swaypath for all tests performed. . . . . . . . . . 185

I.5 Participant 5's swaypath for all tests performed. . . . . . . . . . 186

I.6 Participant 6's swaypath for all tests performed. . . . . . . . . . . 187

I.7 Participant 7's swaypath for all tests performed. . . . . . . . . . . 188

I.8 Participant 8's swaypath for all tests performed. . . . . . . . . . . . 189

I.9 Participant 9's swaypath for all tests performed. . . . . . . . . . . 190

I.10 Participant 10's swaypath for all tests performed. . . . . . . . . . . . 191 
I.11 Participant 11's swaypath for all tests performed. . . . . . . . . . . . 192

I.12 Participant 12's swaypath for all tests performed. . . . . . . . . . . 193 


\section{Nomenclature}

\section{Abbreviations}

AP tilt: anterior-posterior tilt

AVBF system: Abulatory Vibrotactile Biofeedback device used in some studies

BMI: Body Mass Index

CMP: Centroidal Moment Pivot

COM: Center of Mass

COP: Center of Pressure

CPD score: Equitest(R)computerized dynamic posturography test score

ERM: Eccentric Rotating Mass actuator

IMU: Inertial Motion Unit

LRA: Linear Resonant Actuator

ML tilt: medial-lateral tilt

PWM: Pulse Width Modulated

RMS: Root Mean Square

SOT: Sensory Organization Tests, part of computerized dynamic posturography test developed by NeuroCom(REquitest_R 
SPI: Serial Peripheral Interface

VTTF: Vibrotactile Tilt Feedback device used in some studies

ZMP: Zero Moment Point

\section{Definitions}

deadzone: range of tilt magnitudes for which no feedback is provided to the user

force place: force plate on a stationary surface

motion platform: platforms whose motions are controlled by actuators

passive platform: platforms whose motions are reactions to the participant's COM position and tilt angle

tactor: small motor/actuator that vibrates and is used in user feedback applications 


\section{Chapter 1}

\section{Introduction}

\subsection{Motivation}

The world population is aging, in fact, according to Statistics Canada, even though the population growth rate is slowing, the growth rate of older adults has been accelerating. Today, $16.1 \%$ of Canadians are aged 65 and over, which is expected to reach $20.1 \%$ by 2025 and $25 \%$ by 2036 . This increase in proportion brings to the forefront the issues that plague older adult populations. Falls represent one of the leading issues that affect seniors and are defined by the World Health Organization as "an event which results in a person coming to rest inadvertently on the ground or floor or other lower level" [1]. Because of normal physiological changes that accompany the ageing human and the accumulation of multiple medical conditions in some people, falls occur more frequently in this population.

One third of seniors will experience a fall [2] and the rate of falls per 1000 people is consistently increasing [3]. These falls can have devastating impacts on the affected older adults' lives by either resulting in disability, chronic pain or loss of indepen-

dence thereby reducing their quality of life [2], and in an increasing number of cases (increased by $65 \%$ from 2003 to 2008) [3], death.

Apart from how it affects an older adult's life, falls are an expensive preventable 
injury, with an associated total cost of over 3.3 billion dollars in Canada alone in 2010 [4]. For example, fall-related hospitalizations account for about 85\% of all injury hospitalizations for seniors and also demonstrate a much longer mean length of stay (22 days) than other hospitalizations which last on average 12 days [3]. For these reasons, fall prevention strategies and systems are needed, which explains the recent push in research pertaining to fall properties as well as fall prevention devices.

One of the methods commonly used to improve patient balance and thereby reduce falls is biofeedback. There are different types of feedback: auditory, visual and vibrotactile. However, the only type of feedback that can benefit all users is vibrotactile, since some patients lose the ability to balance themselves due to vision loss or with age their hearing abilities have been compromised and can therefore not be relied on to improve balance. Previous studies have shown that vibrotactile feedback related to the wearer's balance state enhances both static and dynamic balance in healthy adults and patients with balance and gait disorders [5].

Though multiple studies have demonstrated the clear effectiveness of vibrotactile feedback to support balance improvement, the development process of the devices used in these studies has been inadequate. Although some research exists to determine the best type of information coding method to be use in balance feedback, no study has compared in depth various feedback locations on the body to determine the feedback location that lends itself to improved balance measures. Instead, the choice of location for feedback in most studies was often not supported or justified [6] [7]. Very little research has been conducted to compare different body locations' ability to sense and parse feedback communicating location and intensity information, thereby potentially reducing the efficacy of vibrotactile feedback devices in existence. Due to the lack of research comparing the effectiveness of vibrotactile feedback depending on the feedback location, and the inability to compare results across studies because of the large inter-study protocol variation, there is a need to conduct a comprehensive 
study on the effects of feedback location on the body.

The Advanced Biomechatronics and Locomotion (ABL) Lab has for some time now investigated and developed multiple systems aiming to allow for patient rehabilitation and retraining in the hopes of providing patients with the independence they one had (in the case of stroke patients) or hope to have (in the case of patients with disabilities). These goals are directly in line with helping older adults remain in independent living situations while also maintaining or improving their quality of life. As previously outlined, fall prevention plays a great role in maintaining quality of life in older adults, and for this reason, the ABL lab has for some time now investigated the possibility of assistive technology in balance improvement under the umbrella of the Balance-Aid Project.

Previous work done by the Balance-Aid group includes the development of a proofof-concept shoe system able to identify a user's balance state using a motion tracking algorithm and pressure sensing insoles to determine if the $\mathrm{CoP}$ of the wearer was within a preset "stable" zone in the polygon of contact with the ground of the wearer. This system, once combined with an adequate feedback device, could allow for balance feedback during ambulatory activities as well as stationary instances. Therefore a feedback device that would provide balance feedback in an easy to understand and sense manner needed to be created for integration with the existing system.

\subsection{Objectives}

Although there is an extensive amount of research conducted on the effectiveness of vibrotactile feedback in improving balance, as aforementioned, there is very little research conducted on the optimization of feedback location, and what research there is only ever compares two body sites. Due to the lack of standardized measures on 
a system's effectiveness in improving balance, results across studies cannot be accurately compared thereby preventing the identification of the best feedback location for balance applications. This work aims to provide a comparison of body locations commonly used in balance feedback applications to determine the best location to be used when communicating balance feedback. In order to do so, the following objectives were set:

1. Determining best tactor type to use in further research conducted in the lab.

The tactor type that is more easily sensed by the user needs to be identified along with the voltage ranges powering it that can be easily sensed.

2. Investigating possible predictors of a person's ability to sense feedback.

Both the participant's Body Mass Index (BMI) and the acceleration data for the tactors were assessed for their ability to accurately predict a person's ability to sense feedback.

3. Comparing 4 body locations based on participant's ability to sense feedback.

This comparison between neck, wrist, waist and ankle is done by analyzing 4 metrics: ability to sense differences in feedback intensity, ability to detect which tactor is activated, ability to detect feedback and reaction time. A weighted sum of each of these parameters' scores was calculated for each body location to allow for comparison.

4. Comparing balance improvement effects for previous test's top scoring locations.

This comparison is completed through analysis of : reaction time, Root Mean Square (RMS) tilt, dominant movement frequency and percentage time spent in the deadzone. Statistical differences between location performance are assessed to determine if there is a meaningful difference between location performance. 
5. Identify protocol changes to improve future studies' quality and effectiveness.

Through designing, conducting and analyzing clinical studies on biofeedback effectiveness, areas in need of improvement or removal from future studies were recorded to improve the quality of future studies conducted in this and other labs.

\subsection{Contributions}

1. Designed three human subjects studies.

Developed study protocols for each study based on a comprehensive review of literature to determine procedures used in similar studies and ensure comparability of results found in this thesis and existing studies. Through study design and implementation, identified changes in protocol and balance measures are recommended for future studies.

2. Determined optimal body site for vibrotactile feedback.

Two studies with 10 and 12 human subjects were conducted to evaluate participants' ability to sense and react to feedback provided on various body sites. A set of performance measures was established based on existing literature to evaluate and compare participant performance across body site. The collected data was processed using SPSS to statistically compare results among body sites and identify the best performing location and the level of significance in differences observed.

3. Experimental equipment development.

To conduct the human subject studies, 3 customized experimental systems were developed. Each one included a microcontroller, two or more tactors, tactor driving circuits, and either an accelerometer or an inertial motion unit (IMU). To collect data using the experimental systems, three Arduino codes were developed 
to communicate with sensors or read from other inputs and activate the tactors accordingly. One of the systems provided real-time feedback on the wearer's trunk tilt.

4. Meta-analyzed and critically reviewed existing studies' results.

Conducted a search to identify articles of relevance, screened articles based on inclusion criteria and extracted results of relevance. Conducted a meta-analysis on results which were comparable across studies and qualitatively analyzed the results that were either not reported consistently across studies or that were reported in too few studies.

Preliminary results from Chapter 3 were presented as part of an abstract presentation titled "Pilot Data on the Performance of Vibrotactile Actuators for Use with Assistive Devices" authored by C. Courtemanche and M. Ahmadi at the 3rd International Conference on Control and Dynamic Systems, and Robotics (CDSR) 2016, on May 11 in Ottawa, Ontario.

\subsection{Overview}

This chapter provides an overview of the work done as part of this thesis as well as the important contributions found in this work.

Chapter 2 is a comprehensive review of the work done in the vibrotactile feedback for balance improvement field, it provides a meta-analysis and qualitative overview of the results found in studies conducted in the field.

Chapter 3 offers a characterization of the two types of tactors considered for use in feedback applications; it helps determine which tactor type should be used in future studies based on ease of sensation and the tactor behavior depending on the body tissue type over which it is mounted. This study also investigates the possibility to 
use accelerometer measurements in predicting the wearer's ability to sense vibrotactile feedback.

Chapter 4 involves the testing of 4 different body sites (wrist, neck, waist and ankle) for performance comparison based on reaction time, ability to detect feedback, ability to identify which tactor was activated as well as its activation intensity. Each tactor location was ranked based on the mean weighted score given to it. Once again the effectiveness of a predictor value based on accelerometer readings of the tactor's vibration characteristics in determining the participants' ability to detect feedback was investigated, this time with a greater sample size than with the previous study. The influence of BMI on a participant's ability to sense feedback was also considered.

Chapter 5 further compares the two best body locations from the previous chapter (waist and ankle) through testing of their effectiveness during balance experiments, which included standing on a hard surface or foam with eyes open or closed to emulate sensory effects experienced by patients with balance impairments. The AnteriorPosterior (AP) and Medial-Lateral (ML) tilt were measured for all trials, along with the reaction time and the percentage time in the deadzone in order to determine if one body location is a significantly better location for balance feedback.

Chapter 6 presents an overview of the main findings associated with this work as well as potential avenues for further research and the plans for future works in the Advanced Biomechatronics and Locomotion Lab. 


\section{Chapter 2}

\section{Literature Review}

\section{$2.1 \quad$ Background}

A person's balance state is dependent on 3 systems in the body: the vestibular, visual and proprioceptive systems. Once one of these systems loses functionality, the person affected will be at an increased risk of falling. Older adults are more likely to experience falls due to known age-related declines in visual acuity, vestibular function and proprioceptive sensitivity [8]. Due to the rapidly increasing older adult population, this increased likelihood of falls has become an imminent problem. These declines in sensory sensitivity mean that there is a lack of information being received by people who are affected, and a method developed to partly replace the decrease in body orientation information is biofeedback. Biofeedback provides sensory information via modalities not usually used to control balance [9]. Vibrotactile biofeedback, feedback provided by vibrating electromechanical actuators (tactors), has been used regularly for balance control and has shown positive effects on balance measures [6] [10]. However, an optimal biofeedback system has yet to be developed since all existing systems have their own limitations.

This review aims to compile the results of previous studies regarding the overall biofeedback effects on anterior-posterior (AP) and medial-lateral (ML) trunk tilt as 
well as the effects that different participant characteristics may have on their response to biofeedback. These results will be compiled using a meta-analysis and qualitative comparisons. Finally this review compares results from the previous studies to determine methods for optimizing biofeedback system design and areas where research is still needed.

\subsection{Methods}

This comprehensive review was conducted on articles found through a search of the databases available through the Carleton University Library (EngineeringVillage, IEEEXPloreDigitalLibrary, JournalofBiologicalEngineering, and BiomedicalEngineeringOnLine). A Boolean search was conducted using various combinations of the words and phrases biofeedback, balance, tactor, vibrotactile, tilt and sway. All articles found under the aforementioned searches were then screened for inclusion in the review, first by title, then by abstract.

\subsubsection{Types of Studies}

All studies found reporting the effects of vibrotactile biofeedback on balance were considered for inclusion. Studies only reporting on biofeedback system development or balance measurement optimization were excluded since they did not report on actual participant balance measures. Studies not including participants, and only considering computer models were excluded from this review; however, studies modeling biofeedback as a control system using measurements recorded for participants receiving biofeedback were included in this review. 


\subsubsection{Types of Participants}

Studies including healthy participants as well as balance impaired patients were included in this review. Healthy participant studies were included seeing as few studies have unhealthy participants and unhealthy participant studies were included since they represent the target population for balance-based vibrotactile biofeedback. Balance impaired patients were people who either had severe vestibular loss, severe balance problems or were weakly compensated vestibulopathic subjects. Studies including amputees were not included in this review due to the large differences in balance caused by prostheses.

\subsubsection{Types of Interventions}

All included studies provided vibrotactile feedback as a means of improving balance. Some studies offered training to their participants with the biofeedback system while others studied the natural responses to biofeedback when no training was provided. Some studies even considered the effects of training using biofeedback by measuring the differences in balance measures pre- and post- training with biofeedback.

Most studies considered the effects of biofeedback in reduced balance situations (as mentioned in the following paragraph) and the remainder of the studies used secondary tasks to affect balance.

Balance measures were taken for a panoply of activities across the studies, including standing normally, standing in a semi-tandem Romberg position, walking normally, walking with a narrowed base, standing on foam, and standing on a tilting platform. To further affect balance, participants were often asked to close their eyes (thereby losing the ability to balance using vision) and/or stand on foam (thereby affecting their proprioceptive sensing abilities) and were therefore assumed to rely more heavily on vibrotactile biofeedback to maintain their balance. 
Two different methods were used to determine biofeedback's effects on balance. The first consisted of determining a group of participants' balance measures without feedback and comparing these values to the ones obtained when these same participants received biofeedback. Another method was to recruit two groups, one that would receive biofeedback and one that would serve as control and compare both groups' values.

\subsubsection{Types of Outcome Measures}

\subsubsection{Primary Outcomes:}

The primary outcomes for this review were the efficacy of biofeedback in improving participant balance and biofeedback system optimization. The efficacy of biofeedback in improving balance was calculated by comparing measures recorded when biofeedback was provided to those recorded when no feedback was provided. Many parameters were used to measure the effect of biofeedback on balance including the trunk tilt angle and velocity, swaypath, center of pressure, linear/angular head displacements and response time. Tilt angle and velocity were measured in both the anterior/posterior and medial/lateral directions, or both measurements were combined to provide a RMS value.

With regards to biofeedback system optimization, most studies incorporated some type of optimization by aiming to determine either the ideal tactor location, sensor location, tactor type or information coding method. All of these parameters are of interest since they dictate the best way of implementing vibrotactile biofeedback. Tactor location optimization is of interest since very few studies have considered this aspect and many studies chose a tactor location based on ease of use of application instead of most accurate means of communication. Sensor location is of interest because the body location for which its motion best describes the likelihood of falling 
has also not been determined as of yet. Many studies also considered various information coding effects, including the methods used to communicate the magnitude and direction of trunk tilt. Balance measures can be communicated using different tactor locations or varying vibration intensity and frequency. For instance the direction of an imminent fall can be communicated using one of two possible types of feedback: attractive (vibrating in the direction towards which the body needs to move to stabilize itself) or repulsive (vibrating in the direction of imminent fall). Some studies even used control models to describe the biofeedback system and would find theoretical solutions to improving the wearer's response to biofeedback.

\subsubsection{Secondary Outcomes:}

The secondary outcomes for this review were not always addressed by all studies, but include age, vestibulopathic, secondary task and naivety effects (in the cases where subjects were not instructed prior to each trial regarding whether feedback would be on or off for the trial, the period of time before feedback was provided in a feedback test was considered the naive period) on biofeedback efficacy in improving balance. The age effects are of interest since the majority of the population in need of a balance aid is comprised of older adults, therefore understanding how the response to biofeedback changes with age allows for the development of balance aids more suited to their target population. The vestibulopathic population response to biofeedback is of interest for the same reasons as the age effects. Most studies only consider the effects of biofeedback on balance when performing activities that allow the user to focus on maintaining their balance; however, in real life, users would be performing a multitude of secondary tasks which would no longer allow them to focus as much on staying upright. For this reason, studies considering secondary tasks effects on biofeedback efficacy are of interest. Naivety is another outcome reported and will be included for completeness, regardless of its impact on practical balance applications. 


\subsubsection{Meta-Analysis}

A meta-analysis was performed using the open-source software OpenMetaAnalyst for any outcome that had 3 or more studies offering comparable data (either a mean difference and associated variance or standard deviation, or both a no feedback and a feedback mean accompanied by their respective variance or standard deviation).

A meta-analysis is used to analytically summarize multiple studies and provide an overall result for the entire sample size that encompasses all studies included thereby increasing the power to identify effects of interest [11]. A meta-analysis can also help resolve controversies introduced by conflicting studies by providing an overall estimate of the effect of an intervention or treatment [12].

The overall mean for each outcome meta-analyzed is found by taking the weighted mean of the results found in each study. To decrease the effect of differences in study interventions on the results of the meta analysis, the differences observed between control (no feedback) and biofeedback cases were taken for all the studies. By taking the difference, the intervention effects on the measures taken should be diminished or removed since they would be present in both groups and only the improvement due to feedback should be present in the differential data. Of course, this is not a perfect method, but it is the only feasible means of comparing data available. The procedure used to calculate the overall mean and its $95 \%$ confidence interval (CI) is outlined below.

The overall mean for $\mathrm{n}$ studies, mean $_{s}$, which takes into account each study mean is calculated as [13]:

$$
\text { mean }_{s}=\frac{\sum_{i=1}^{i=n}\left(\text { weight }_{i} \cdot \text { mean }_{i}\right)}{\sum_{i=1}^{i=n}\left(\text { weight }_{i}\right)}
$$


where mean $_{i}$ is the mean difference observed for study $i$ found as:

$$
\text { mean }_{i}=\text { mean }_{\text {feedback }}-\text { mean }_{\text {nofeedback }}
$$

and weight $t_{i}$ is the weight associated with the study based on its variance, calculated as:

$$
\text { weight }_{i}=\frac{1}{\text { variance }_{i}}=\frac{1}{S D_{i}^{2}}
$$

Using the pooled variance, variance , the $95 \%$ confidence interval for the combined data can be found as:

$$
95 \% C I=\text { mean }_{s} \pm\left(1.96 \cdot \text { variance }_{s}^{0.5}\right)
$$

where the pooled variance is calculated as:

$$
\text { variance }_{s}=\frac{1}{\sum_{i=1}^{i=n}\left(\text { weight }_{i}\right)}
$$

The data presented by the meta-analysis provides $95 \%$ confidence intervals and means. To allow for determination of significance of results' differences, the standard deviations were found using the conversion [14] in Equation 2.6.

$$
S D=\frac{U L-L L}{3.92}
$$

Where SD is the standard deviation, UL is the upper limit of the $95 \%$ confidence interval and LL is the lower limit. 


\section{$2.3 \quad$ Results}

\subsubsection{Summary of Studies}

A summary of the key characteristics of each of the studies reviewed is provided in Tables 2.1 to 2.13 to allow a greater understanding of each study's biofeedback system (including tactor and sensor placements) and intervention. Studies are ordered by study ID, which consists of the main author's last name and year of publication.

It is important to note that two biofeedback systems are used in multiple studies. The first system, named the Vibrotactile Tilt Feedback Device (VTTF), has 16 columns and 3 rows of tactors equally spaced around the wearer's torso vibrating at a frequency of $250 \mathrm{~Hz}$ with a 3 axis IMU placed on the lower back as a trunk tilt sensor. The system is designed to work on the principle of repulsive biofeedback and communicates an increasing tilt magnitude by activating a tactor in a higher row.

The second system consists of 2 subsystems. The SwayStar is the sensing component of the system, which is comprised of a 2 axis IMU placed on the participants' lower backs. The BalanceFreedom is the system component that provides biofeedback. It has 3 intensities, for the lowest intensity of trunk tilt, one of the 8 tactors positioned around the head is activated, for the second level of intensity one of 2 boneconducting acoustic transducers on the mastoids is activated, and for the greatest tilt intensity one of 2 LEDs is activated. There is no mention of the tactor vibration frequency but the acoustic frequencies range between 250 and $1370 \mathrm{~Hz}$. The vibrotactile biofeedback is repulsive for this system. 
Table 2.1: Davis 2010 Study Design

\begin{tabular}{|c|c|}
\hline Methods & Randomized to intervention or control \\
\hline Participants & $\begin{array}{l}32 \text { healthy young adults aged } 30 \text { to } 33 \text { years }+32 \text { healthy } \\
\text { community-dwelling older adults aged } 58 \text { to } 77 \text { years }\end{array}$ \\
\hline System & SwayStar and BalanceFreedom \\
\hline Intervention & $\begin{array}{l}\text { Both groups had their balance performance initially assessed for } 12 \\
\text { differences stances without biofeedback } \\
\text { Intervention: } 1 \text { week later, received biofeedback during balance } \\
\text { training and post-test } \\
\text { Control: } 1 \text { week later, received balance training and performed } \\
\text { post-test without biofeedback }\end{array}$ \\
\hline Outcomes & $\begin{array}{l}\text { Balance performance differences by age, differences between pre and } \\
\text { post intervention ML and AP trunk tilt angle }\end{array}$ \\
\hline Measurement & ML and AP tilt angles measured using IMU \\
\hline
\end{tabular}

Table 2.2: Goodworth 2011 Study Design

\begin{tabular}{ll}
\hline Methods & $\begin{array}{l}\text { Only one group; all subjects performed tests with and without the } \\
\text { system active } \\
\text { patients with severe vestibular loss aged } 36 \text { to } 63 \text { years }\end{array}$ \\
Participants & $\begin{array}{l}\text { VTTF device limited to } 1 \text { direction of motion (AP motion } \\
\text { communicated with tactor columns on stomach and back) }\end{array}$ \\
Intervention & $\begin{array}{l}\text { Subjects received no training and stood on AP pseudorandom tilting } \\
\text { platforms with eyes closed for tactors on and tactors off conditions }\end{array}$ \\
Outcomes & $\begin{array}{l}\text { COM (center of mass) sway, system effectiveness in reducing sway as a } \\
\text { function of stimulus frequency and amplitude }\end{array}$ \\
Measurement & $\begin{array}{l}\text { COM measured using platform force plate, sway measured using } \\
\text { accelerometers / gyroscopes of sensor }\end{array}$ \\
\hline
\end{tabular}


Table 2.3: Haggerty 2012 Study Design

\begin{tabular}{ll}
\hline Methods & $\begin{array}{l}\text { Only one group; all participants performed } 2 \text { trials for each experimental } \\
\text { procedure combination }\end{array}$ \\
Participants & 10 healthy community-dwelling older adults aged 68 to 80 years \\
System & $\begin{array}{l}4 \text { tactors on a belt (on stomach, back and sides) (signal proportional to } \\
\text { trunk tilt angle, tactor closest to direction of tilt vibrated); } 2 \text { axis IMU } \\
\text { placed on the lower back measuring AP and ML trunk tilt; Vibration } \\
\text { beat of } 20 \mathrm{~Hz} \text { (obtained with } 2 \text { square waves of } 250 \text { and } 270 \mathrm{~Hz} \text { ); } \\
\text { Repulsive biofeedback }\end{array}$ \\
Intervention & $\begin{array}{l}\text { Subjects trained with system, experimental conditions included: stance } \\
\text { (normal vs. semi-tandem Romberg), visual condition (eyes open vs. }\end{array}$ \\
& $\begin{array}{l}\text { closed), feedback (on, off) and secondary task (none, verbal or } \\
\text { push-button identification of tone pitch played) }\end{array}$ \\
Outcomes & $\begin{array}{l}\text { Response time, \% time in deadzone, effects of naivety on response time } \\
\text { IMU measured tilt, response time measured by computer which recorded } \\
\text { push-button voltage and voice responses }\end{array}$ \\
\hline
\end{tabular}

Table 2.4: Janssen 2010 Study Design

\begin{tabular}{|c|c|}
\hline Methods & $\begin{array}{l}\text { Randomized to Ambulatory Vibrotactile Biofeedback (AVBF) or } \\
\text { reference group }\end{array}$ \\
\hline Participants & $\begin{array}{l}10 \text { patients aged } 45 \text { to } 76 \text { years in } \mathrm{AVBF} \text { group }+10 \text { patients aged } 40-65 \\
\text { years in reference group, all having severe balance problems with } \\
\text { frequent falls }\end{array}$ \\
\hline System & $\begin{array}{l}\text { AVBF system: Tactors on belt (normal mode- only } 1 \text { tactor activated, } \\
\text { full mode- all tactors activated if head/trunk tilt exceeds threshold; } \\
\text { random mode- } 1 \text { tactor activated regardless of tilt direction/magnitude); } \\
3 \text { IMU on head or trunk measure tilt; vibrates at } 300 \mathrm{~Hz} \text {; Repulsive } \\
\text { biofeedback }\end{array}$ \\
\hline Intervention & $\begin{array}{l}\text { AVBF group: system familiarization, stood with feet at hip width on } \\
\text { foam covered force platform without biofeedback; with sensor on head } \\
\text { and tactors in normal, full and random modes; and with sensor on trunk } \\
\text { and tactors in normal, full and random modes (eyes were closed) } \\
\text { Reference group: performed } 4 \text { trials as above, but with no feedback }\end{array}$ \\
\hline Outcomes & Body sway angle, swaypath, swayarea and mean sway (AP and ML) \\
\hline Measurement & Body sway angle measured using force platform (CoP and body length) \\
\hline
\end{tabular}


Table 2.5: Lee 2013 Study Design

\begin{tabular}{ll}
\hline Methods & Only one group; all participants performed the same tests \\
Participants & 11 healthy young adults aged 20 to 29 years \\
System & 6 tactors placed on belt at L4/L5 height (on left/right internal oblique, \\
& external oblique and erector spinae), 2 types of tactors: Tactaid \\
& (electromagnetic inertial transducer) and C2 (voice-coil type linear \\
& actuator) (no method to distinguish trunk tilt magnitude); 3axis IMU \\
& on lower back measured angular position/velocity/acceleration in AP \\
& and ML directions; Vibrates at 250 Hz, peak to-peak displacements of \\
& 200 um (C2) and 50 um (Tactaid); measures natural body responses to \\
& biofeedback; No training \\
& Subjects stood eyes closed and feet hip-width apart on firm surface, no \\
& training, single location feedback (only 1 tactor vibrates at a time) vs. \\
& all locations feedback (all tactors vibrate at same time), 6 single \\
Intervention & locations and 1 all locations trials per tactor type per subject \\
& AP and ML RMS sway, postural shift vector, PSD of AP and ML sway \\
Outcomes & Sway measured as angular displacements in AP and ML directions using \\
Measurement & IMU
\end{tabular}

Table 2.6: Peterka 2006 Study Design

\begin{tabular}{ll}
\hline Methods & Allocated to healthy or vestibular loss group based on diagnosis \\
Participants & $\begin{array}{l}6 \text { healthy subjects with mean age of } 35 \pm 8 \text { years }+5 \text { severe vestibular } \\
\text { loss subjects with mean age of } 52 \pm 11 \text { years }\end{array}$ \\
System & $\begin{array}{l}4 \text { vertical columns of } 3 \text { tactors (2 on anterior and } 2 \text { on posterior lower } \\
\text { torso) (each pair showed a different trunk tilt range, tilt magnitude } \\
\text { proportional to tactor height); } 1 \text { axis IMU attached to backboard forcing } \\
\text { movement in AP direction measured tilt and tilt velocity; Vibration } \\
\text { intensity set to highest current rating (200 mA); Repulsive biofeedback }\end{array}$ \\
Intervention & $\begin{array}{l}\text { Subjects stood on a motion platform and were held by a backboard on } \\
\text { hinges so they could only move in AP direction, pseudo-random motion } \\
\text { platform was activated }\end{array}$ \\
Rutcomes & RMS body sway, transfer function gains \\
Measurement & Sway measured by IMU mounted on backboard \\
\hline
\end{tabular}


Table 2.7: Sienko 2008 Study Design

\begin{tabular}{|c|c|}
\hline Methods & Only one group; all participants performed the same tests \\
\hline Participants & $\begin{array}{l}8 \text { weakly compensated vestibulopathic subjects (failed the } \\
\text { NeuroCom(REquitest@ (R) computerized dynamic posturography Sensory } \\
\text { Organization Tests (SOT) } 5 \text { and } 6 \text { ) aged } 32 \text { to } 67 \text { years }\end{array}$ \\
\hline System & $\begin{array}{l}\text { VTTF device: looked at } 4,8 \text { and } 16 \text { column configurations with AP } \\
\text { and ML presented as a vector (only } 1 \text { tactor on at a time) and at } 4 \\
\text { column configuration with AP and ML trunk tilt presented } \\
\text { independently ( } 2 \text { tactors can be on at same time) }\end{array}$ \\
\hline Intervention & $\begin{array}{l}\text { Subjects stood on tilting pseudo-randomly translated platform with } \\
\text { embedded force plate, subjects trained prior to study, eyes closed for all } \\
\text { trials, pre/immediately-post / } 20 \text {-minutes post feedback tests for no } \\
\text { feedback conditions, erroneous feedback provided to subjects (placebo } \\
\text { effect), } 1 \text { day to } 2 \text { months later subjects performed no feedback and } 16 \\
\text { column configuration tests }\end{array}$ \\
\hline Outcomes & $\begin{array}{l}\text { RMS trunk tilt, elliptical fits to sway trajectory areas, percentage of } \\
\text { time outside deadzone, RMS CoP }\end{array}$ \\
\hline Measurement & $\begin{array}{l}\text { Tilt measured by IMU, tilt magnitude (square root of the squared sums } \\
\text { of roll and pitch) }\end{array}$ \\
\hline
\end{tabular}

Table 2.8: Sienko 2010 Study Design

\begin{tabular}{ll}
\hline Methods & Only one group; all participants performed the same tests \\
Participants & 8 weakly compensated vestibulopathic subjects aged 32 to 67 years \\
\hline System & VTTF device: looked at 4,8 and 16 column configurations with AP \\
& $\begin{array}{l}\text { and ML presented as a vector (only } 1 \text { tactor on at a time) and at } 4 \\
\text { column configuration with AP and ML trunk tilt presented } \\
\text { independently (2 tactors can be on at same time-1 for AP tilt and } 1 \text { for } \\
\text { ML tilt) }\end{array}$ \\
\hline Intervention & $\begin{array}{l}\text { Stood on a motion platform able to move in } 2 \text { orthogonal directions, } 6 \\
\text { trials with eyes closed (trials with tactors off, with } 4 \text { tactor } \\
\text { configurations [4 (ML and AP presented together), } 4 \text { (ML and AP }\end{array}$ \\
& $\begin{array}{l}\text { presented independently), } 8 \text { columns, } 16 \text { columns], second trial with } \\
\text { tactors off) }\end{array}$ \\
& $\begin{array}{l}\text { PSD functions of body sway in AP and ML directions, transfer function } \\
\text { between platform motion and body sway }\end{array}$ \\
Measurement & $\begin{array}{l}\text { PSD functions of body sway measures and platform velocity, transfer } \\
\text { functions and coherence functions relating platform motion and trunk } \\
\text { tilt }\end{array}$ \\
\hline
\end{tabular}

\subsubsection{Meta-Analysis of Results}

There are few meta-analysis results due to the lack of comparability of data from different studies. Only the overall biofeedback effect on AP and ML tilt could be metaanalyzed, where the overall biofeedback effect on tilt refers to the difference in tilt 
Table 2.9: Tino 2011 Study Design

\begin{tabular}{|c|c|}
\hline Methods & Only one group; all participants performed the same tests \\
\hline Participants & 3 healthy subjects aged 40 to 70 years \\
\hline System & $\begin{array}{l}3 \text { tactors in each glove on palms and forearms (increasing degree of trunk } \\
\text { tilt indicated by number of tactors activated, when tilting sideways, only } \\
\text { one side will vibrate, when tilting in AP direction both forearms } \\
\text { vibrate); } 3 \text {-axis IMU on the subject's back measured linear and angular } \\
\text { torso accelerations; Tactors vibrate at } 200 \mathrm{~Hz} \text {; Repulsive biofeedback }\end{array}$ \\
\hline Intervention & $\begin{array}{l}\text { AP smooth or jerking accelerations provided to waist and torso, tests } \\
\text { were done with and without biofeedback and with eyes closed }\end{array}$ \\
\hline Outcomes & Tilt recovery, response time \\
\hline Measurement & Tilt and response time measured using the accelerometers \\
\hline
\end{tabular}

Table 2.10: Verhoeff 2009 Study Design

\begin{tabular}{ll}
\hline Methods & Only one group; all participants performed the same tests \\
Participants & $\begin{array}{l}13 \text { healthy elderly (older adults) mean age of } 70.8 \pm 2 \text { years }+16 \\
\text { healthy young subjects mean age of } 21.5 \pm 0.7 \text { years }\end{array}$ \\
System & $\begin{array}{l}\text { SwayStar and BalanceFreedom devices } \\
\text { Intervention }\end{array}$ \\
$\begin{array}{l}\text { Performed } 3 \text { tasks with and without one of } 2 \text { secondary tasks (carrying } \\
\text { tray with filled plastic glasses or subtracting sevens), performed initial } \\
\text { assessment to establish biofeedback thresholds, trained to use } \\
\text { biofeedback, performed } 3 \text { tasks with biofeedback this time }\end{array}$ \\
Outcomes & $\begin{array}{l}\text { Trunk sway angle and velocity, effects of feedback/age/cognitive } \\
\text { task/motor task on performance }\end{array}$ \\
Measurement & $\begin{array}{l}\text { Motor task performance measured by amount of water spilled, trunk tilt } \\
\text { measured using IMU }\end{array}$ \\
\hline
\end{tabular}

experienced by subjects when receiving biofeedback and when performing the control intervention. This lack of meta-analyzed data is due to the fact that only 4 studies published enough information to determine the biofeedback effect on AP tilt [15] [16] [17] [18] and similarly only 3 studies provided enough information to determine biofeedback effects on ML tilt [10] [16] [15]. Because the remaining parameters (e.g. the effect of tactor location, sensor location,...) were only investigated by 2 or less studies or were reported in different manners across studies, a meta-analysis of the data pertaining to these parameters could not be conducted and qualitative analysis and discussion of these results was performed. This lack of data to compare using 
Table 2.11: Wall 2001 Study Design

\begin{tabular}{ll}
\hline Methods & Only one group; all participants performed the same tests \\
Participants & 6 healthy participants aged 22 to 29 years \\
System & $\begin{array}{l}\text { One tactor on each shoulder (head tilt magnitude indicated by pulse } \\
\text { repetition rate) or } 2 \text { vertical strips of } 7 \text { tactors on lateral torso } \\
\text { (increasing tilt magnitude indicated by higher tactor activated); } 3 \text { axis }\end{array}$ \\
& $\begin{array}{l}\text { IMU head-mounted IMU senses tilt; } 250 \mathrm{~Hz} \text { vibration frequency; } \\
\text { Repulsive biofeedback }\end{array}$ \\
Intervention & $\begin{array}{l}\text { Familiarization with the balance aid systems, patients stood in } \\
\text { semi-tandem Romberg position with eyes closed and tried to maintain } \\
\text { balance with no aid (control), shoulder tactors active, torso tactors }\end{array}$ \\
& $\begin{array}{l}\text { active and light touch (lightly touching a stable object in front of them), } \\
\text { No training }\end{array}$ \\
RMS head-tilt angle, RMS CoP displacement (RMS CPD) and fraction \\
of time over threshold (FOT)
\end{tabular}

Table 2.12: Wall 2005 Study Design

\begin{tabular}{ll}
\hline Methods & $\begin{array}{l}\text { Allocated to one of two groups depending on the Computerized } \\
\text { Dynamic Posturography (CDP) score }\end{array}$ \\
Participants & $\begin{array}{l}9 \text { patients aged } 27 \text { to } 68 \text { years }+8 \text { patients aged } 23 \text { to } 61 \text { years with } \\
\text { CPD scores greater than } 45 \text { and less than } 45, \text { respectively (varying } \\
\text { balance dysfunction between groups) }\end{array}$ \\
System & $\begin{array}{l}\text { VTTF device limited to } 1 \text { direction of motion (AP motion } \\
\text { communicated with tactor columns on stomach and back) }\end{array}$ \\
Intervention & $\begin{array}{l}\text { Subjects stood feet shoulder-width apart on platform providing AP } \\
\text { perturbations with movable visual surround, subjects trained to vary }\end{array}$ \\
& $\begin{array}{l}\text { CoP using VT feedback, Sensory Organization Test (SOT) } 5 \text { and } 6, \\
\text { Equitest MCT medium rear and large rear perturbations }\end{array}$ \\
RMS trunk tilt, RMS CoP, \% falls, learning effects, biofeedback effects, \\
age effects
\end{tabular}

a meta-analysis indicates that there is a need for further research into the effect of these parameters on tilt, but also that there is a lack in standardization of reporting methods and measures in the biofeedback field.

When considering the effectiveness of vibrotactile feedback in reducing tilt, and improving balance, it was found that biofeedback caused a decrease in AP and ML 
Table 2.13: Wall 2009 Study Design

\begin{tabular}{|c|c|}
\hline Methods & $\begin{array}{l}\text { Only one group; participants performed tests with and without the } \\
\text { system active }\end{array}$ \\
\hline Participants & 12 healthy patients aged 65 to 90 years with perceived balance problems \\
\hline System & VTTF device \\
\hline Intervention & $\begin{array}{l}\text { Dynamic Gait Index (DGI) test, walking trials (eyes open normal } \\
\text { stance, eyes closed normal stance, eyes open narrow stance, eyes closed } \\
\text { narrow stance) }\end{array}$ \\
\hline Outcomes & $\begin{array}{l}\text { Scores on the DGI, differences in temporal and spatial characteristics of } \\
\text { gait, and amount of ML trunk tilt obtained with and without the VTTF }\end{array}$ \\
\hline Measurement & $\begin{array}{l}\text { DGI performed by physiotherapist board, kinematic analysis done with } \\
\text { motion capture system }\end{array}$ \\
\hline
\end{tabular}

tilt in all studies. The mean (lower, upper 95\% CI) meta-analyzed decreases in AP and ML tilt are $-0.815(-1.285,-0.418)$ degrees and $-0.332(-0.581,-0.083)$ degrees, respectively, where the negative values indicate a decrease in tilt when feedback is provided as opposed to when no feedback is provided. The mean decrease in AP tilt is significantly larger $(\mathrm{p}<0.01)$ than the mean decrease in ML tilt due to biofeedback, showing that biofeedback not only effectively reduces the tilt measured in participants, but also has a significantly greater effect on AP tilt than on ML tilt.

\subsubsection{Comparison of Non-Meta-Analyzed Results}

\subsubsection{General Biofeedback Effects}

Of all the studies included in this review, no study showed evidence of an overall significant increase in tilt caused by biofeedback and therefore no study showed statistically significant adverse effects associated with biofeedback. In addition to the meta-analyzed studies which showed decreases in tilt using feedback, Janssen 2010 showed that only some of the patients affected with severe balance problems experienced a significant decrease in tilt with biofeedback; however, the entire study's population still exhibited a significant mean decrease in tilt. As mentioned in the 
meta-analysis of results, many studies observed significant decreases in the AP direction but much smaller or non-significant decreases in the ML direction [6] [16]. It was also observed that participants who would have initially larger ranges of angular displacements before the use of biofeedback would show the largest tilt reductions with the use of biofeedback [15].

One study examined the effects of erroneous feedback (tactors activated in directions unrelated to the tilt direction) to ensure that the sheer presence of vibrations did not cause an improvement in tilt, but that directional feedback needed to be provided in order to improve tilt [6]. This study showed that erroneous feedback gave significantly higher tilt values than when no biofeedback and directional biofeedback were provided and that there needs to be a direction associated with the vibrations in order to improve tilt.

\subsubsection{Age Effects}

Only 3 studies reported the effects of age on the user's response to vibrotactile biofeedback and on balance measures in general. Regarding balance deficits, Davis 2010 found that older adults, in the age range of 58-77 years old, exhibited greater amounts of ML and AP trunk tilt in one-legged stance, tandem walking and walking while rotating head than the younger adults in the study. These results were supported by Wall 2005, who found that AP tilt and peak tilt increase with age both with and without biofeedback. However, Wall 2005 did find ameliorations in CoP and recovery time with age regardless of the biofeedback state.

No other study explicitly reported on the differences in balance between ages; they instead focused on the improvement observed due to biofeedback. Regarding ML tilt, Davis 2010 found that ML trunk tilt decreases due to biofeedback for the tandem stances and normal walking were greater for the younger subjects than the older ones and there were no other significant differences observed. This observation 
is supported by Wall 2005 and in part by Verhoeff 2009. Wall 2005 noted that the VTTF helped the younger subjects reduce their body motion better than it helps the older subjects. Similarly, Verhoeff 2009 remarked greater reductions in AP trunk tilt for younger subjects during dual tasking, however, the older adults were the only ones experiencing a decrease in tilt during normal walking. Although the direction of tilt in which there is a larger improvement varies between studies, which can be attributed to the fact that studies do not all look at the same directions of tilt, it can be observed that the older adults benefit less from biofeedback than do their younger counterparts when performing secondary tasks, but they sometimes are the only ones showing balance improvements in simple tasks when biofeedback is provided.

\subsubsection{Secondary Task Effects}

When looking at the effects of secondary tasks on balance and response to biofeedback, two major parameters were considered: the response time and the effect on tilt. All of the studies determining the effects of dual tasking on response time found an increase in response time allocated to the presence of a secondary task, regardless of the task. For example, Haggerty 2012 found an increase in response time when biofeedback was provided while trying to identify the pitch (high or low) of a noise being played; this was thought to be due to the de-prioritization of the secondary task (identifying the pitch). Verhoeff 2009 found that the duration of trials for which secondary tasks (walking a given distance while counting backwards or balancing water-filled cups on a tray) were performed in conjunction with biofeedback was greater than when no biofeedback was provided. This increase in task duration might also be linked to an increase in response time, however, the study's authors seemed to think it had more to do with the decreased angular velocity which would in turn decrease the walking speed, but no justification for this decrease in angular velocity was given.

Both of the above studies also found that the performance of a secondary task 
causes balance measures to improve. For instance, Verhoeff 2009 found that when walking while balancing a tray with water-filled cups, participants' trunk sway significantly decreased. Haggerty 2012 found agreeing results stating that the trunk tilt significantly decreased with biofeedback; in addition, it was found that the percentage of time spent in the deadzone (range of tilt for which no feedback is provided and the participant is considered balanced) increased when secondary tasks were performed whether or not biofeedback was provided. From the studies reviewed, the performance of secondary tasks brings an increase in the percentage time spent in the deadzone, a decreased trunk tilt and an increased reaction or response time.

\subsubsection{Balance Impairment Effects}

It was found that biofeedback helped significantly reduce trunk tilt in all studies considering the effects on balance-impaired participants. Wall 2005 found that the no-fall AP tilt was significantly reduced with biofeedback for participants with low Computerized Dynamic Posturography scores, which are identifiers of balance problems. Wall 2009 is in agreement, finding a significant decrease in overall trunk tilt. Peterka 2006 also found significant decreases in trunk tilt with biofeedback, but by comparing the body tilt values to those of healthy participants, it was determined that biofeedback could not restore a normal level of body tilt in vestibulopathic participants.

Biofeedback could also have negative impacts on the user if they had vestibulopathic problems. Wall 2005 found that for participants with very bad CPD scores (worse balance dysfunctions) biofeedback significantly decreased the percentage of falls and for participants with a slightly better CPD score, biofeedback significantly increased the percentage of falls. This study also found that biofeedback had no significant effect on CoP. Although Wall 2009 showed significant and positive effects of biofeedback on support and tilt velocity for normal stance, the results for a narrow 
stance with the eyes closed showed an increase in tilt velocity and a decrease in support. It is evident that although trunk tilt is significantly decreased when participants with balance dysfunctions receive biofeedback, other parameters are worsened by the use of biofeedback. It is therefore very important to conduct thorough testing of a balance aid system before subjecting patients with balance dysfunctions to its use. It should also be noted that, depending on the system, the negative impacts could have a varying range of severity associated with them.

\subsubsection{Naivety Effects}

Only Haggerty 2012 reported on the effects of naivety on balance measures. The only significant effect observed is an increase in response time once the participant loses his/her naivety and becomes aware that the device can provide biofeedback. This is believed to be due to an increased cognitive load caused by the presence of biofeedback.

\subsubsection{System Optimization}

There is a panoply of biofeedback system aspects that could be improved, however, very little research has be conducted on an in-depth analysis of system optimization methods. Some studies consider parts of an aspect, but never explore it fully. Some of the most important design parameters that can be altered include the sensor location, the tactor location, the tactor type and the method for coding tilt direction (attractive vs. repulsive) and magnitude.

Sensor location: Only one study considered the effects of sensor location on biofeedback efficacy [19]. It was found that more patients showed significant improvements on swaypath during biofeedback when the sensor was placed on the head as opposed to the trunk. No other possible sensor locations have been compared or considered. 
Tactor location: Only one study has looked at the effects of tactor location on biofeedback efficacy, unfortunately the study decided to also change the type of information coding for tilt with each location; meaning that the magnitude of tilt was communicated by the pulse rate for the shoulder location and by the height of the tactor activated for the waist location [10]. In doing so, the study prevents the accurate comparison of locations or of tilt coding methods since there is no way to distinguish the effects that the location and the coding method each have on the tilt magnitude.

Information Coding: Some studies did however correctly compare different tilt coding methods. Sienko 2008 compared 4 configurations made up of 4, 8 or 16 columns with 3 rows for intensity. Although no significant tilt differences were observed for the 16-column configuration, many participants felt that they were receiving too much information using this configuration and were feeling overwhelmed. Arguably, although no measurable effects were found to this effect, the 16 column configuration should probably not be used since ease of use is an important aspect when designing technology and even more so if the target population contains older adults. In 2010, Sienko conducted another study which represented the body's responses to biofeedback using a control system and found that there was a lack of consistent differences between PSD plot for different configurations which indicated that tactor spatial resolution has no effects on sway reduction or frequency bandwidth for which sway is reduced. Although both studies employed very different methodologies, they came to the same conclusion that tactor spatial configuration bears no effect on the efficacy of the device. It must be noted that both studies used tactors on the trunk only, and thus other locations might behave differently.

In Sienko 2008, there were also 2 types of configurations for the 4 column set-up, one combined AP and ML tilt so that only 1 tactor at a time was vibrating in the direction closest to the tilting direction whereas the other configuration presented 
AP and ML tilt independently which meant that 2 tactors could vibrate at the same time. No optimal tactor configuration emerged from this study, however the 4-column configuration which presented AP and ML tilt independently produced the greatest tilt out of all the tactor configurations. It can therefore be understood that the combination of AP and ML measurements when communicating tilt direction is the optimal method of presenting both AP and ML tilt.

With regards to tactor type, one study compared C2 (voice-coil type linear actuators) and Tactaid (electromagnetic inertial transducer) tactors' effects on natural body sway [7]. It was found that the $\mathrm{C} 2$ tactors created greater postural shift vector magnitudes, possibly because of its greater stimulus amplitude.

Lee 2013 also investigated the natural body reactions to biofeedback when no training was provided. It was found that, depending on the vibration location, movement in the direction of the vibration could be observed. This study only looked at tactor locations on the torso; for the tactors on the internal obliques and erector spinae, movements in the direction of vibration were observed, however for the external obliques, no significant movement was observed. This might be because the latter requires purely ML movement whereas movement in the internal oblique and erector spinae direction would be AP and are known to have a greater magnitude and range of motion as aforementioned. Unfortunately, there is too large a latency in postural shift with respect to vibration stimulus and so the reaction observed may have nothing to do with reflexes, but instead may be dictated by some other, slower means, which is unknown.

\subsubsection{Functional Bandwidth and Stimulus Properties}

Although making use of balance measures like trunk tilt is a commonly accepted method to determine the effects of biofeedback on the balance state, some studies analyzed the effects of biofeedback as if it were part of a control system. This control 
system had a platform stimulus as its input and the participants' trunk tilt as its output, which meant the gains were the ratio of body tilt to platform tilt. In doing this analysis, some of the studies determined the ranges of functionality of the biofeedback systems as well as methods for improving said systems. For instance, the critical platform stimulus frequency was determined as being the frequency for which any frequency smaller than the critical frequency would be linked to a decrease in gains and for stimulus frequencies greater than the critical frequency, an increase in gain would be observed. A decrease in gain represents a decrease in body tilt for the same stimulus amplitude or a constant amount of tilt even with increasing stimulus amplitude, which is desirable.

Goodworth 2011 found gain reductions due to biofeedback at frequencies up to $0.4 \mathrm{~Hz}$ in its participants who all had severe vestibular loss. Similarly, Peterka 2006 found that for its participants with severe vestibular loss, biofeedback only provided a decrease in gains for stimulus frequencies up to $0.5 \mathrm{~Hz}$. Peterka 2006 compared these results to those of healthy participants and found that biofeedback can be effective over a larger range of frequencies for healthy subjects than for subjects with vestibular loss since the critical frequency for healthy subjects was $0.8 \mathrm{~Hz}$. Contrary to the lower frequency ranges observed in Peterka 2006 and Goodworth 2011, Sienko 2010 observed gain reductions in AP trunk tilt for vestibulopathic subjects for stimulus frequencies up to $2 \mathrm{~Hz}$. This is a large difference from the other 2 studies since all 3 studies looked at AP trunk tilt in vestibulopathic subjects and there were very little differences between the biofeedback systems employed in all 3 studies, but Peterka obtained values more than twice as large as the other two studies. From these results it is possible to denote that although biofeedback is effective in reducing tilt at low stimulus frequencies, it can actually have a detrimental effect at higher stimulus frequencies.

Sienko 2010 also found that the stimulus bandwidth for which biofeedback is 
useful and not detrimental is dependent on the stimulus direction. For AP trunk tilt, a greater usable bandwidth has been found (up to $2 \mathrm{~Hz}$ ) whereas for ML tilt the bandwidth is considerably smaller (up to $0.6 \mathrm{HZ}$ ). This study also found that the usable bandwidth is independent of tactor configuration.

The other major factor explored in these control models of biofeedback is the effect of stimulus amplitude on gain. Goodworth 2011 showed that as stimulus amplitude increased, the vibrotactile feedback was less effective in reducing postural sway for vestibulopathic patients whereas another study demonstrated an increase in gain reduction with an increase in stimulus amplitude for healthy subjects [20]. Peterka 2006 found this same discrepancy between healthy and vestibulopathic participants. Through modeling responses to biofeedback as a control system it was found that, although tilt may decrease in vestibulopathic participants when biofeedback is provided, biofeedback was shown to not fully restore balance for vestibulopathic patients.

\subsection{Discussion}

\subsubsection{Meta-Analysis}

The meta-analysis showed statistically significant decreases in AP and ML tilt with biofeedback, thereby demonstrating that biofeedback is effective in improving balance. The ML decreases were significantly smaller than the AP decreases; this is likely due to the fact that people are more stable in the ML direction than the AP direction and there is thus more improvement to be seen in the AP direction.

\subsubsection{Age effects}

The reason why younger subjects performed better than the older adults when given biofeedback during dual tasks might be due to the decrease in residual processing 
capacity with age. As for the older adults being the only ones benefiting from biofeedback in normal walking, this might be due to the fact that the older adults have much higher amounts of tilt normally than their younger counterparts and would therefore show the potential for more improvement in tilt using biofeedback.

\subsubsection{Secondary task effects}

When looking at secondary task effects, it is not surprising that a task focused on balancing objects should lead to a decrease in trunk sway because the task requires an added focus on balance which requires not only that the tray be balanced but that the person holding it have a more balanced state. Thus, the participants are actively focusing on their balance, but also obtaining a form of visual feedback since the cups' orientation which is analogous to their body orientation can be easily observed when looking at the angle made between the water level and the cup's surface. Interestingly enough, even when a dual task is not aimed at balancing an object (i.e. identifying the pitch of a sound) the participants' tilt would also decrease, for which no logical explanation can be derived. The fact that the reaction time increases with the presence of secondary tasks is expected and shown in the two studies regarding secondary tasks, however the increased amount of time in the deadzone is an unexpected result for a standing trial test. One would think that when focusing on a secondary task, the participants would be focusing less on balancing themselves and would therefore have a decreased balance state, which is not actually the case. Some studies have suggested that the increased time in the deadzone due to biofeedback might be due to participants tensing up when receiving biofeedback, which decreases their tilt and places them in the deadzone [6]. 


\subsubsection{Balance impairment effects}

It was observed that for participants with balance dysfunctions a varying range of effects could be observed, although in all cases the amount of tilt was significantly decreased. These results put into question whether or not tilt is the best measure of balance because even with the significantly reduced tilt, some participants were more likely to fall with biofeedback or experienced greater tilt velocities, which could subject them to greater risks of falling [17]. A combination of measures might be a better method to predict the likelihood of falls and the efficacy of a system in improving balance. Interestingly enough, many studies used trunk tilt as the measure to drive the biofeedback, but reported the effects of biofeedback on other measures. As would be expected, the effects on the other measures are often not as desired due to this lack of connection between the measure used to provide feedback and the measure recorded as an estimate of balance. For instance, it was found that for Wall 2005 the trunk tilt decreased significantly with biofeedback, but there was no significant change in $\mathrm{CoP}$ which could in part be due to the fact that $\mathrm{CoP}$ was not used as an input for the biofeedback. Alternatively, this could be due to the fact that CoP motions could be oscillating at a rapid frequency thereby exhibiting weakness and preventing significant differences from being observed across trials because of the greater intra-trial variation, a behavior that would be filtered out of the tilt signal (integrating the angular velocity to obtain the tilt angle effectively low-pass filters the signal).

\subsubsection{Naivety effects}

There is very little research on the effect of naivety, which is arguably the less important parameter to be discussed when considering biofeedback effects on balance since participants using biofeedback to balance themselves would not be naive to the 
presence of biofeedback. Interestingly enough, Haggerty 2012 came to a very similar conclusion as Verhoeff 2009 although both studies made use of different systems and found different results. Both studies came to the conclusion that the main issues arising from the use of biofeedback stemmed from an increased cognitive load due to biofeedback met by a reduced processing capacity due to age effects. This assumed increase in cognitive load due to biofeedback is supported by Goodworth 2011 who found that participants maintained the same reliance on proprioceptive information with and without biofeedback and used the vibrotactile information as additive and not substitutive information. Therefore, when biofeedback is provided, more information is provided leading to an increase in cognitive load. This goes to support why healthy participants considered to have an ideal balance can also benefit from biofeedback use; the information provided through biofeedback is added to that of their own sensory system thereby improving their balance perception since they have the cognitive ability to interpret the entirety of the information provided to them.

\subsubsection{System Optimization}

There are still many questions that remain unanswered by the existing literature regarding vibrotactile biofeedback effects on balance. There is no research on the effects of long-term biofeedback training on balance and whether or not the biofeedback devices are only useful for a certain amount of time before users become desensitized or if their efficacy increases continuously as the users become more accustomed to them. A major optimization aspect that has been very poorly researched so far is the tactor location; there is a need for a study that could compare the effects of multiple (more than 2) tactor locations on balance to find the ideal tactor location.

There is also very little research and conflicting results between existing studies regarding whether or not repulsive biofeedback is a more ideal means of conveying tilt information than attractive biofeedback. Only one study reported on the natural 
body responses to vibrotactile biofeedback, however there was a very limited range of locations tested. It would be interesting to know if the body naturally tilts towards the direction of vibrations regardless of the tactor location. It would also be useful to explore if the component (muscle, bone or fat) on which tactors are placed have an effect on natural body sway responses; if some cause greater magnitudes of sway or if the sway directions vary based on the biomaterial subjected to vibration. Lastly, vibrotactile biofeedback also inadvertently provides auditory biofeedback since the vibrations are often audible; it would be interesting to quantify the effects on tilt associated with auditory as opposed to vibrotactile biofeedback.

Interestingly enough, Janssen 2010's study showed that the optimal sensor location would be on the head; however, only one out of the 12 other studies actually had the sensor on the head. This seems to be the trend in this field, since much of the research published goes unused by groups in the same field. This may be largely attributed to the large number of variations between designs, but also to the fact that a gold measurement standard has yet to be set. This means each group has determined their preferred measures of balance and methods for obtaining these measures which prevents the translation and comparison of results from one group to another. There is a real need for more consistency in reporting and measuring balance for this emerging field of research.

Furthermore, additional research into determining the optimal tactor location and information coding method needs to be conducted since no study has completed a thorough comparison of all possible body locations. It seems that most studies chose body locations and tilt coding methodologies based on intuition rather than factual information. Although no spatial configuration effect was found for tactors on the torso, it does not mean that this is the case for all body locations and so, this aspect too should be considered for various body locations. 
Once again, although an optimal tactor type was determined, there is very unlikely going to be a change in tactors used across the board because of the lack of communication between groups. It is also impossible to compare this result to the ones of other studies because the tactors for the other studies are not described in the same way.

Although one study researched the natural body sway in response to biofeedback, it was only concerned with the reaction to biofeedback on the torso, it would be interesting to see if all locations behave the same way. Interestingly enough, this study showed that the natural body response to vibration is to move in the direction of the vibration (which is analogous to attractive biofeedback); however, all other systems reviewed made use of repulsive biofeedback. There should definitely be some thought put into the creation of a system that works with the body's natural response as opposed to trying to override the body's natural impulses.

\subsubsection{Functional Bandwidth and Stimulus Properties}

When considering the control model of a biofeedback system it became very apparent that biofeedback was only useful for lower stimulus frequencies, especially in patients with vestibular loss. This is an expected result since the vestibular system operates over a greater bandwidth than the vibrotactile biofeedback can. Therefore when trying to convey tilt information using biofeedback (which is low pass filtered by the receptors in the body) only the low frequency information can be communicated, whereas the body requires the high frequency information to effectively replace the vestibular information loss. Thus, when stimulus frequencies increase past a critical point, some of the information is no longer communicated to the user who is unaware of this change and more tilt can result due to biofeedback. Although the qualitative behavior is known, more research has to be conducted to determine the exact stimulus bandwidth for which biofeedback creates decreases in gains, since the current studies 
on the matter have similar qualitative data, but varying quantitative data.

\subsubsection{Future Directions}

A major question raised through the use of control systems to model biofeedback is whether or not the measures currently used to determine the efficacy of biofeedback systems are truly representative of the wearer's balance state. For instance, should gains or tilt differences be used universally as balance improvement measures?

When considering the effects of biofeedback on balance measures, is it more beneficial for all participants to see the same decrease in tilt or should the target decrease be scaled to the initial amount of tilt experienced by the wearer thereby having a larger effect on people with more severe balance problems than those with lesser balance dysfunctions? Although using a ratio to ensure that those with a greater need for reduction in tilt experience the greater improvements with biofeedback would seem like the better way to represent the effectiveness of the biofeedback system, very few studies made use of this measure, perhaps this should become a standard evaluation metric for future biofeedback systems' effectiveness, especially when comparing patient to healthy participant effects.

\subsection{Summary of Findings}

Biofeedback effectively decreases body tilt for lower stimulus frequencies and although it can be helpful for patients with vestibular loss it in no way fully compensates for the loss of vestibular information. Although the current systems show improvements in tilt magnitude in both the ML and AP directions, there are many aspects of these systems that could be optimized including the sensor location, feedback location, the use of attractive tilt coding and tilt magnitude coding; a summary of the main findings associated with each biofeedback optimization and balance effects parameter 
can be found in Table 2.14.

Since this is an emerging field, there are still no best practices for reporting and no measurement methods established which significantly affects the ability to compare results from one study to another and to allow for the application of one group's research discoveries to another group's. A consistent way for measuring the effectiveness of a biofeedback system in decreasing tilt should be developed and a set of testing procedures should be performed by all of the research groups in this field. 
Table 2.14: Summary of Review Findings

\begin{tabular}{|c|c|c|}
\hline Parameter & Studies & Findings \\
\hline $\begin{array}{l}\text { Balance } \\
\text { Impairment }\end{array}$ & [17] [21] [18] & $\begin{array}{l}\text { - Biofeedback significantly reduces tilt in all studies } \\
\text { - Biofeedback does not always restore patient tilt to healthy } \\
\text { participant level } \\
\text { - Feedback causes fall percentage to decrease for poor CPD } \\
\text { score patients and to increase for better CPD score patients }\end{array}$ \\
\hline $\begin{array}{l}\text { Feedback } \\
\text { Location }\end{array}$ & {$[10]$} & $\begin{array}{l}\text { - Compared waist and shoulder using different information } \\
\text { coding methods per location } \\
\text { - No results could be associated with feedback location alone }\end{array}$ \\
\hline $\begin{array}{l}\text { Sensor } \\
\text { Location }\end{array}$ & [19] & $\begin{array}{l}\text { - Significant improvements in swaypath using biofeedback } \\
\text { were more common when sensor was on the head as } \\
\text { opposed to the trunk }\end{array}$ \\
\hline $\begin{array}{l}\text { Information } \\
\text { Coding }\end{array}$ & {$[10][6][22][7]$} & $\begin{array}{l}\text { - Varying number of tactor columns on the waist showed no } \\
\text { significant performance difference } \\
\text { - Presenting ML and AP tilt in conjunction reduces tilt more } \\
\text { than providing feedback on ML and AP tilt independently } \\
\text { - Tactor spatial resolution bears no effect on sway reduction } \\
\text { - Linear actuators produced greater postural shift than } \\
\text { electromagnetic inertial transducers }\end{array}$ \\
\hline $\begin{array}{l}\text { Functional } \\
\text { Bandwidth }\end{array}$ & [23] [21] [22] & $\begin{array}{l}\text { - A stimulus frequency for which feedback no longer } \\
\text { improves tilt exists, but varied across studies } \\
\text { - Healthy participants benefited from feedback over wider } \\
\text { stimulus bandwidth than patients } \\
\text { - The useful feedback bandwidth is greater in AP than ML } \\
\text { direction }\end{array}$ \\
\hline Age & {$[15][16][17]$} & $\begin{array}{l}\text { - No clear effect of age on overall performance } \\
\text { - Elderly benefit less from biofeedback when performing } \\
\text { secondary tasks than younger participants } \\
\text { - Elderly are sometimes the only ones exhibiting balance } \\
\text { improvements using biofeedback in simple tasks }\end{array}$ \\
\hline $\begin{array}{l}\text { Secondary } \\
\text { Task }\end{array}$ & {$[24][16]$} & $\begin{array}{l}\text { - Increase in response time and percentage time spent in the } \\
\text { deadzone as well as a decrease in tilt when secondary task } \\
\text { is present, regardless of biofeedback state }\end{array}$ \\
\hline
\end{tabular}




\section{Chapter 3}

\section{Tactor Vibration Characterization}

\section{$3.1 \quad$ Objective}

In order to eventually compare the effectiveness of providing feedback on various body locations, it is important to first understand the mechanical properties associated with the feedback to be provided and to determine which type of actuator should be used in feedback applications. There is very little in the way of research on which type of tactor is best sensed by the wearer; the only study involved in this type of research found that voice-coil type actuators were more easily sensed than electromagnetic inertial transducers [7]. There is also no research conducted on the ability to predict

whether or not participants will be able to sense feedback, an ability which could prove useful when first setting-up a feedback device for a user; it could allow for customization of the device to ensure that all vibrations provided to the user could be felt.

To address these shortcomings in current research, this study was designed with three main goals:

1. Characterize and develop a fuller understanding of each tactor type's vibration properties 
2. Further research the ideal tactor type and its operational range for use in feedback applications by comparing the performance of a voice-coil actuator to a different kind of tactor (an eccentric rotating mass actuator)

3. Establish a quantitative predictor of participant's ability to sense biofeedback

Furthermore, by researching the ideal tactor type in an independent study, later studies conducted with greater participant groups will have less tests, making the research process more efficient. In addition, characterizing the vibrations for each tactor could be a useful tool in ensuring that tactors are performing as expected during future studies.

\subsection{Vibration Sensing}

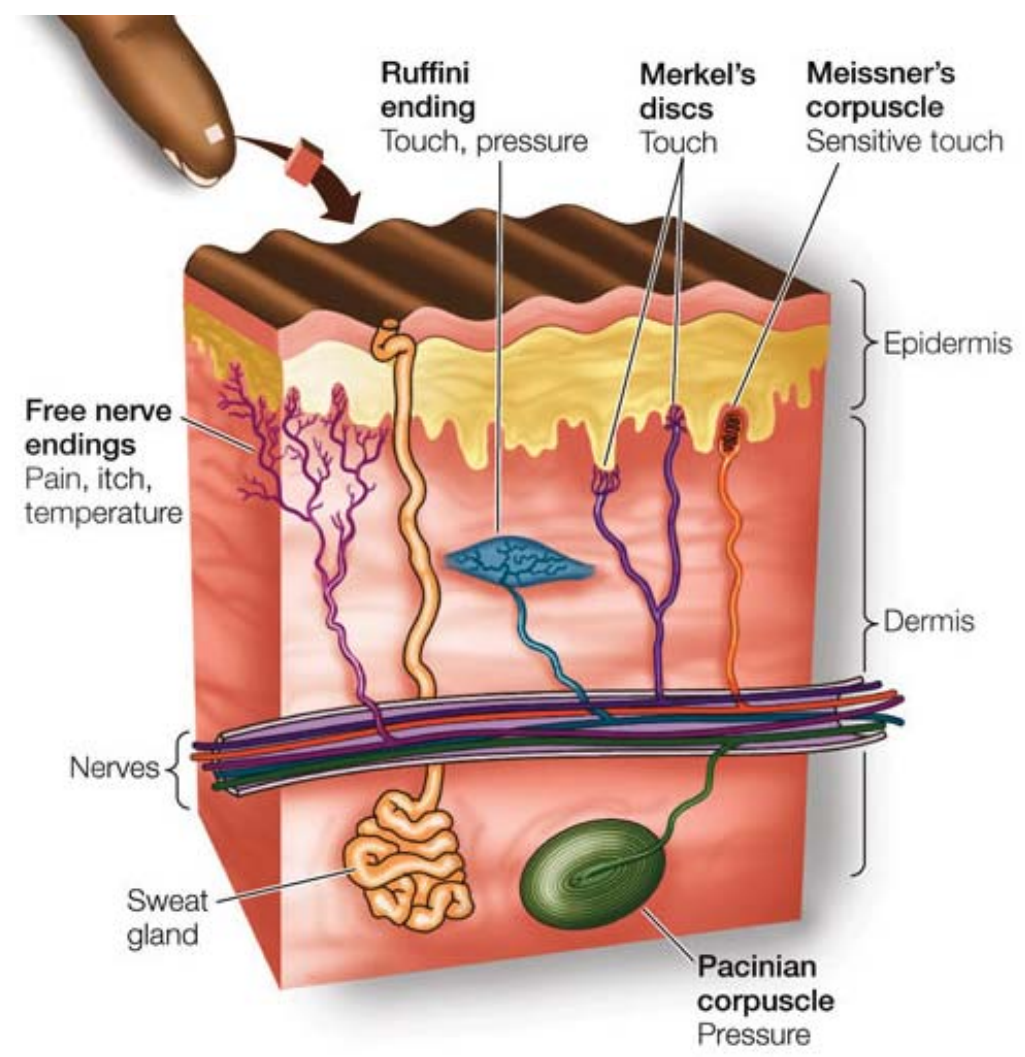

Figure 3.1: Depiction of mechnoreceptor shape, size and location within the skin [25]. 
To understand participants' ability to sense feedback and the effects that different types of feedback mechanisms can have on sensation, some background knowledge pertaining to the sensing elements of the skin is necessary. There are 4 main sensing elements (shown in Figure 3.1) in the skin that provide information to the central nervous system with regards to pressure, touch, vibration and skin tension [26]. Each sensing element senses a different mechanical stimulation type and frequency as outlined in Table 3.1.

Table 3.1: Types of stimulus sensed by each type of mechanoreceptors

\begin{tabular}{|c|c|c|c|}
\hline $\begin{array}{c}\text { Mechanoreceptor } \\
\text { Type }\end{array}$ & $\begin{array}{l}\text { Stimulation } \\
\text { Frequency } \\
\text { Sensed }\end{array}$ & $\begin{array}{c}\text { Type of } \\
\text { Mechanical } \\
\text { Stimulus Sensed }\end{array}$ & $\begin{array}{l}\text { Adaptation } \\
\text { Rate }\end{array}$ \\
\hline $\begin{array}{l}\text { Pacinian } \\
\text { Corpuscles }\end{array}$ & $10-800 \mathrm{~Hz}[27]$ & Vibrations [28] & $\begin{array}{c}\text { Rapidly } \\
\text { adapting [26] }\end{array}$ \\
\hline $\begin{array}{l}\text { Meissner's } \\
\text { Corpuscles }\end{array}$ & $10-200 \mathrm{~Hz}[27]$ & $\begin{array}{l}\text { Slip/low frequency } \\
\text { vibrations }[28]\end{array}$ & $\begin{array}{c}\text { Rapidly } \\
\text { adapting [26] [28] }\end{array}$ \\
\hline Merkel's Discs & $0.4-100 \mathrm{~Hz}[27]$ & $\begin{array}{c}\text { Pressure/ } \\
\text { indentation [28] }\end{array}$ & $\begin{array}{c}\text { Slowly } \\
\text { adapting [28] }\end{array}$ \\
\hline Ruffini Endings & $7 \mathrm{~Hz}[27]$ & Skin stretch [28] & $\begin{array}{c}\text { Slowly } \\
\text { adapting [26] }\end{array}$ \\
\hline
\end{tabular}

Table 3.1 defines the Pacinian corpuscles as the main sensing element for vibrations on the skin. Although, they can sense vibrations over a wide range of frequencies (10-800 Hz), at frequencies outside the optimal sensitivity range of $150-250 \mathrm{~Hz}$ much larger vibration magnitudes are needed to allow vibration detection, as shown in Figure 3.2 which depicts the current opinion in neurobiology with respect to sensation to vibrations. For instance, when considering feedback within the optimal frequency range, vibrations of magnitudes as small as $0.02 \mathrm{~mm}$ can be felt whereas for vibrations of $10 \mathrm{~Hz}$ in frequency, a minimum vibration amplitude of over $10 \mathrm{~mm}$ needs to be provided to the user in order to be felt by the Pacinian corpuscles. Furthermore, the 
threshold for Pacinian corpuscle sensitivity to vibrations on the skin varies depending on the vibration's orientation with respect to the skin; Pacinian corpuscles have lower sensation thresholds for vibrations parallel to the skin as opposed to those perpendicular to the skin [28].

The Meissner's corpuscles sense slip and low frequency vibrations which are often not detected by the Pacinian corpuscles. Although they have been shown to be two orders of magnitude less sensitive to tactile feedback than Pacinian corpuscles [28], they are the most common receptor of the glaborous skin [26] and could therefore greatly impact a person's ability to sense vibrotactile feedback, especially for low vibration frequencies nearer their optimal sensitivity range of 30-50 $\mathrm{Hz}$ [26].

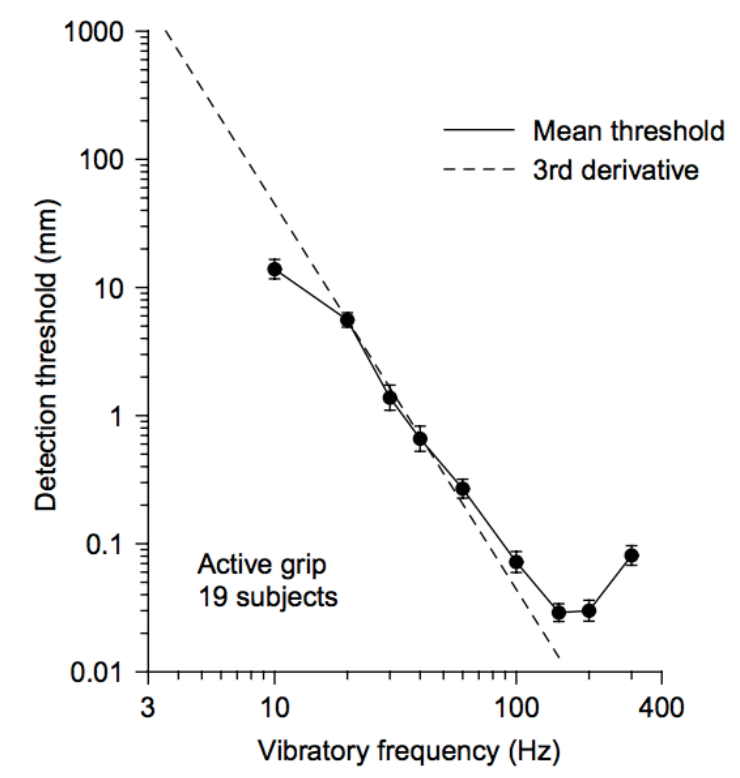

Figure 3.2: Pacinian corpuscle's minimum vibration displacement for detection as a function of vibration frequency, taken from [28].

Lastly, the Merkel's discs and the Ruffini endings, due to their sensitivity primarily to low frequency mechanical stimulations as well as their sensitivities to stimulations other than vibrations, are less likely to play a role in a participant's ability to sense vibrotactile feedback, but are included in this review for completeness [29]. 


\subsection{Methods}

\subsubsection{Subjects}

The participant population in this study was a sample of convenience made up of subjects between 23 and 25 years of age, two participants were female and one was male. Participant demographics are outlined in Table 3.2. The primary inclusion criterion was that subjects were healthy with no reported neurologic conditions and thereby able to sense vibrotactile feedback on various body locations.

Table 3.2: Participant Demographics

\begin{tabular}{lc}
\hline Variable & Value \\
\hline Gender & \\
Male & 1 \\
Female & 2 \\
Age Range & $23-25$ \\
Age Mean (STD) & $24(1)$ \\
BMI Range & $19.5-28.2$ \\
BMI Mean (STD) & $24.0(4.4)$ \\
\hline
\end{tabular}

\subsubsection{Procedure}

Participants underwent 6 trials (testing for tactor vibration over lipid, muscular and bony tissue for two tactor types). For each trial, participants underwent 5 test series; for each test series, the tactor of interest was powered with 7 different voltages and the participants verbally identified their ability to detect the tactor vibrations. Five test series were conducted for each tactor/body tissue combination in order to provide a mean behavior for each of the measures of interest. The testing order for each participant was randomly assigned to prevent testing order effects on results. 
For each test, the tactor of interest was powered at the required voltage, during which time the acceleration profile of the tactor in the $\mathrm{x}, \mathrm{y}$ and $\mathrm{z}$ directions was recorded. For each voltage, participants stated whether or not they felt vibration feedback; these comments were recorded in binary form (1 if they sensed the vibration feedback and 0 if they did not) for use in identifying the tactor type to use in future studies. The measures of interest for tactor characterization and feedback detection prediction were calculated offline following the tests based on the acceleration profile recorded.

\subsubsection{Experimental Setup}

The equipment used in this study included two types of tactors onto which a three dimensional accelerometer (the ADXL 345) was attached using PeelnStick, a permanent adhesive. The accelerometers were powered by an Arduino Mega 2560 board. This Arduino board also controlled the vibration intensity of the tactors by sending a Pulse Width Modulated (PWM) signal to a Texas Instruments DRV 2603 chip that would output either a DC or AC voltage proportional to the PWM signal's duty cycle in order to power the tactors.

\subsubsection{Tactor Selection}

Two different types of tactors were tested in this study in order to determine which type should be used in further research stages. The two tactor types in question were the Eccentric Rotating Mass (ERM) actuator and the a Linear Resonant Actuator (LRA). Both tactors chosen were purchased from Precision Microdrives, a supplier that creates vibration actuators designed for haptic feedback.

The ERM, more specifically the 310-003 tactor, was chosen for its larger vibration amplitude than competing ERMs for any given voltage while maintaining a current draw small enough (max. start current of $120 \mathrm{~mA}$ ) to be supplied by the Arduino 


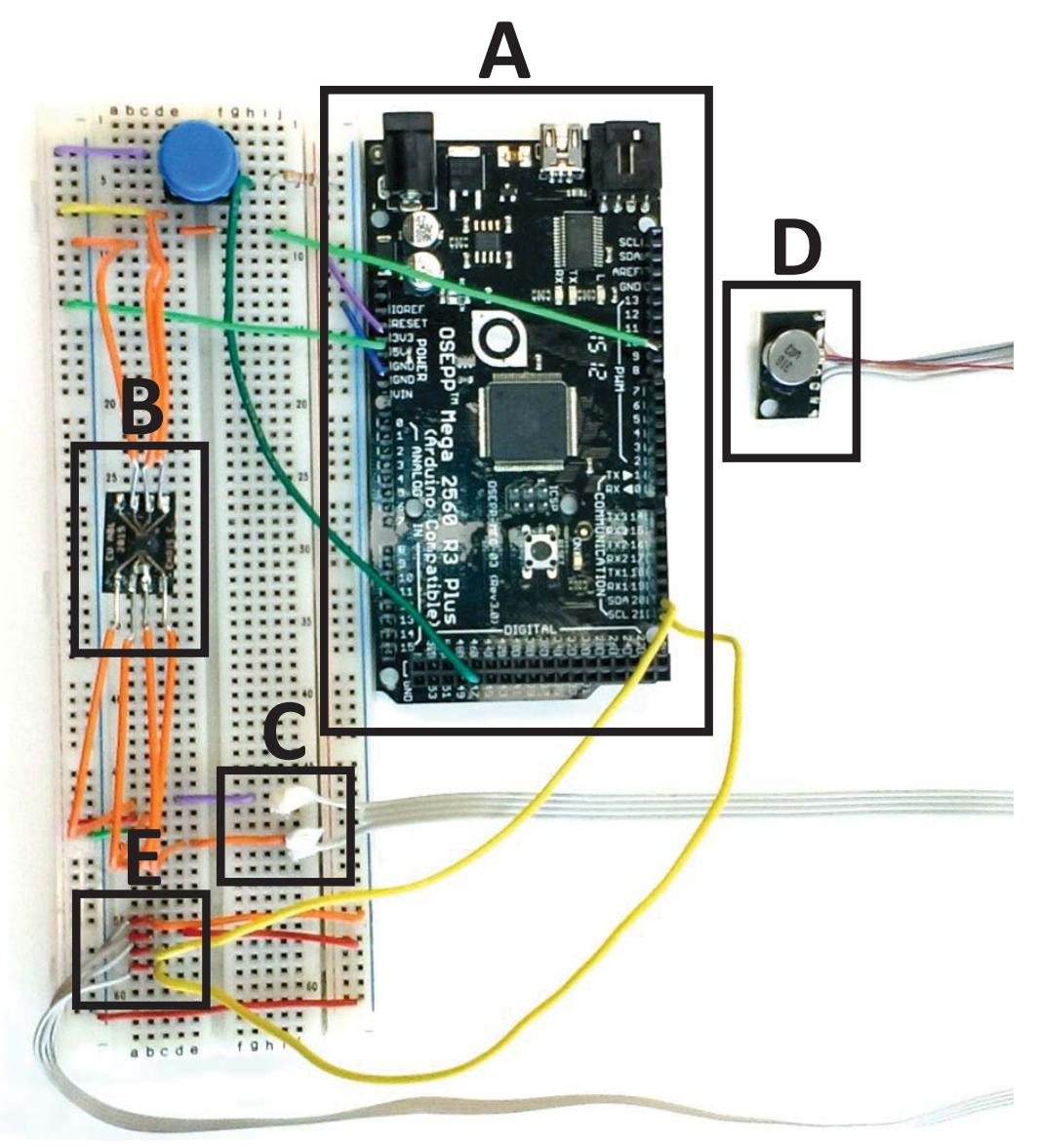

Figure 3.3: Circuit and its components used in this study : A) Arduino Mega 2560 board, B) TexasInstruments DRV 2603 chip, C) tactor powering wires, D) tactor and accelerometer assembly, E) accelerometer communication wires

board and Texas Instruments DRV 2603 chip. The ERM is a DC motor with an offset mass attached to its shaft, which causes an imbalance in the centripetal forces leading to the tactor movement parallel to the plane on which the tactor is attached as can be seen in Figure 3.4 [30] [31].

Figure 3.5 shows the datasheet performance curves for the ERM used in this study, and it can be seen that both the vibration acceleration amplitude and the vibration frequency vary as a function of voltage, both of which will be monitored to ascertain if they have any effect on participant's ability to sense feedback.

The second tactor type was the C10-000 tactor; a LRA with the same surface area as the chosen ERM, so as to have the possibility to innervate the same number of 


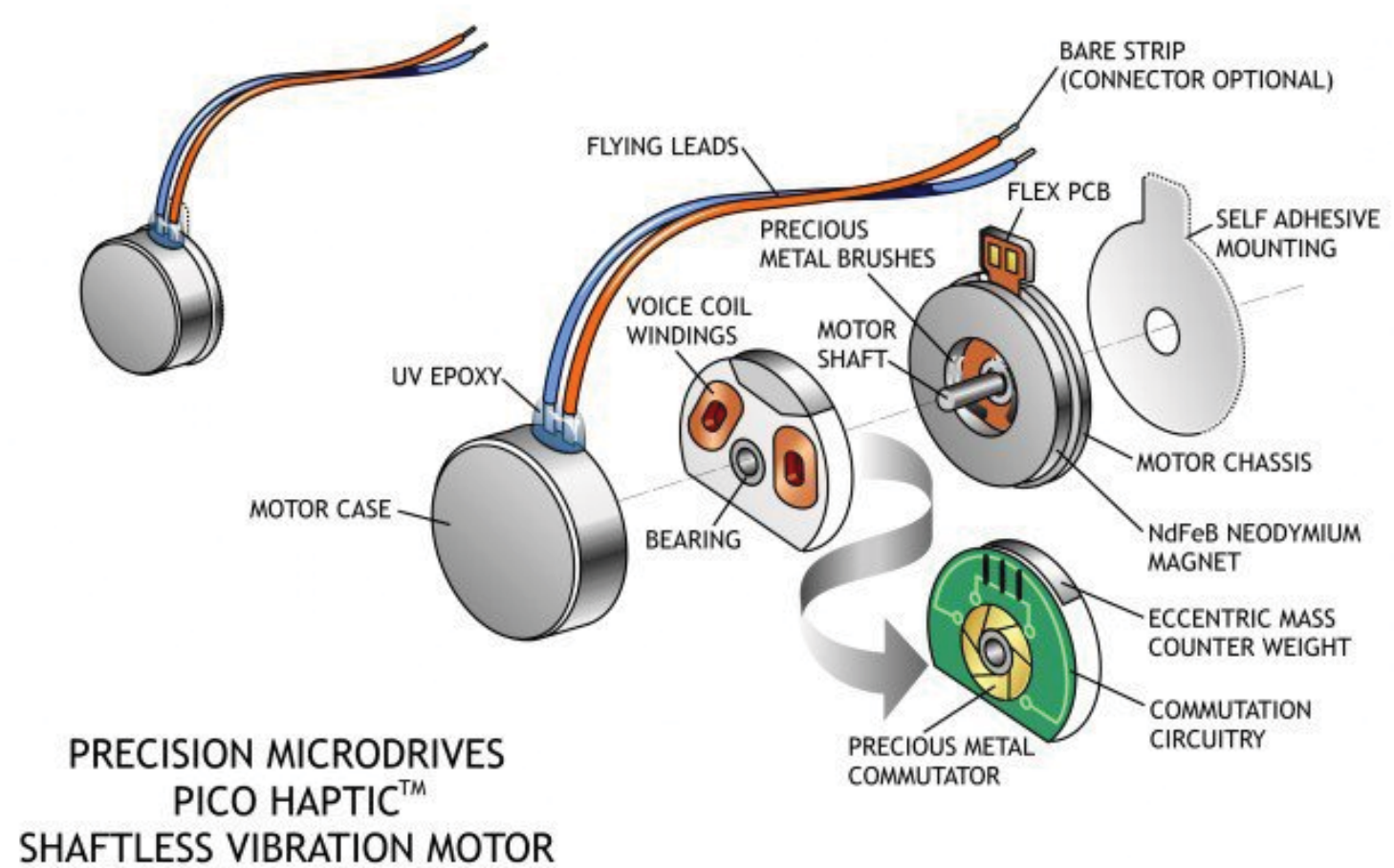

Figure 3.4: Part breakdown of the ERM actuator as shown on the Precision Microdrives web page

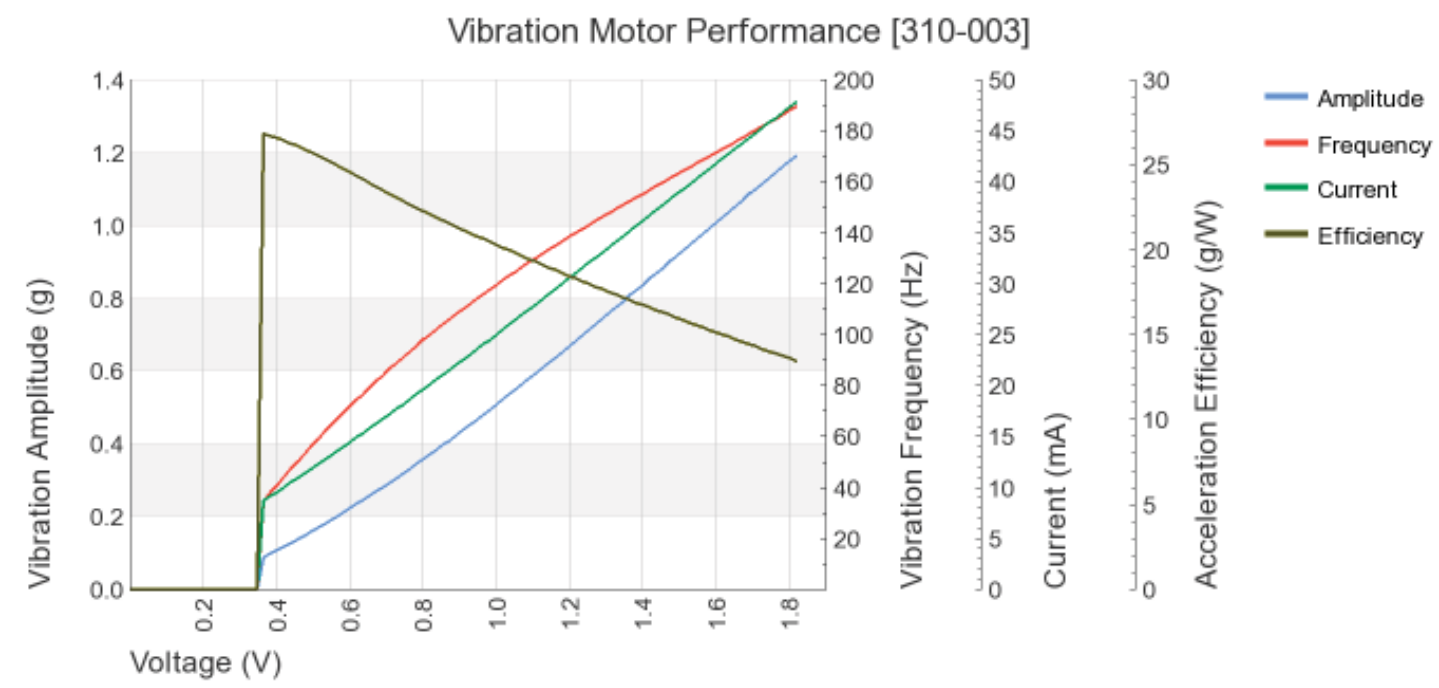

Figure 3.5: 310-003 tactor datasheet performance.

mechanoreceptors. The LRA actuator creates vibrations through the use of a voice coil drive which generates a magnetic field causing a magnet and mass suspended on a spring to oscillate. The mass in this case moves up and down, causing vibrations 
perpendicular to the surface on which the tactor lies [32]. The construction of the LRA can be observed in Figure 3.7. Furthermore, LRAs must be powered by an AC signal whose frequency matches within a few Hertz the resonant frequency of the LRA, otherwise the vibration amplitude of the LRA is drastically reduced as shown in Figure 3.6. To complicate matters, the LRA's resonant frequency is dependent on the surface onto which the LRA is mounted [33].

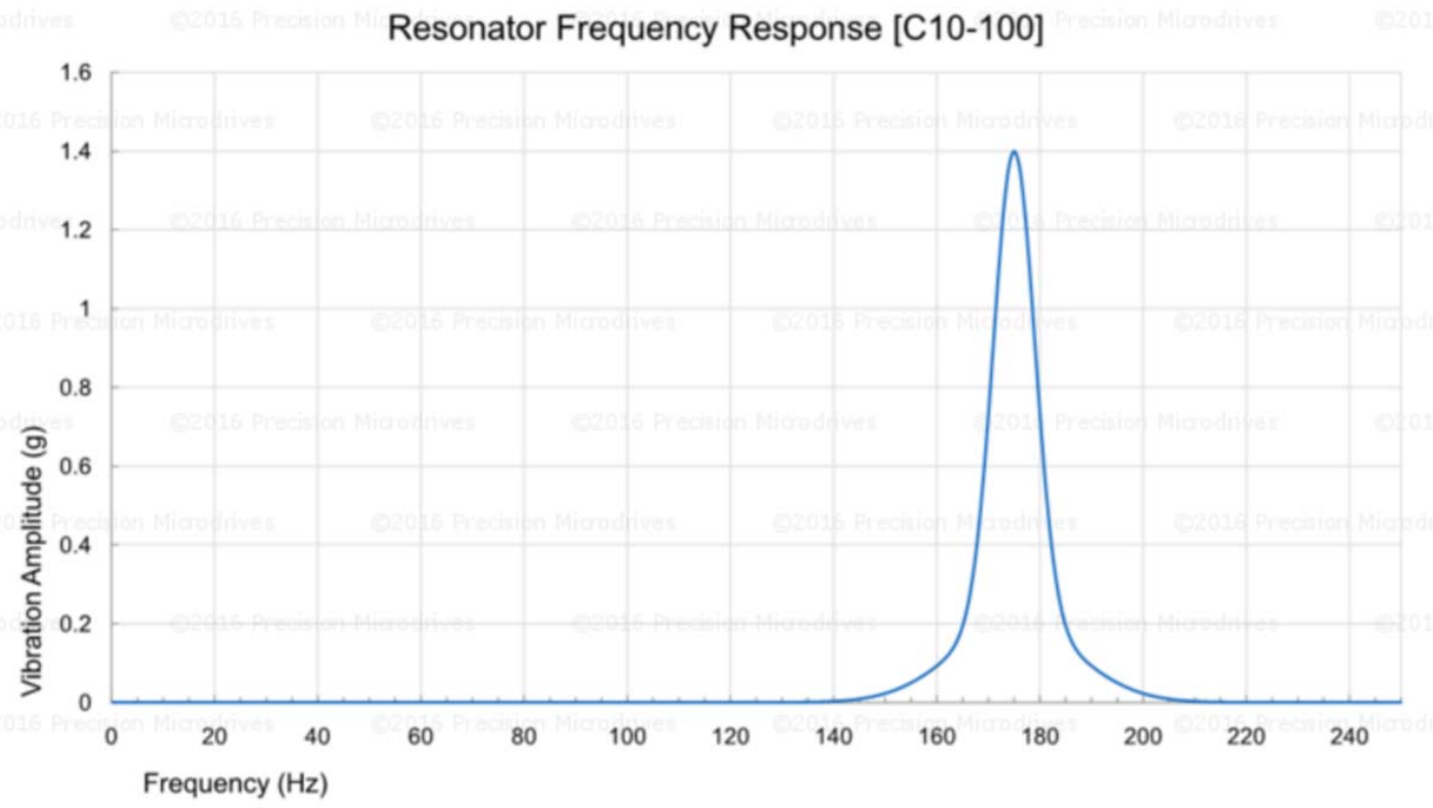

Figure 3.6: LRA vibration amplitude as a function of the AC voltage frequency powering it [33].

This particular LRA was chosen because it had the greatest vibration acceleration amplitude for any given voltage, while drawing a current small enough (max. operating curent of $90 \mathrm{~mA}$ ) to be powered by the Arduino and Texas Instruments DRV 2603 chip. Figure 3.8 shows the datasheet performance curves for the LRA used in this study.

Based on Figures 3.5 and 3.8, the C10-000 (LRA) tactor would be expected to yield more noticeable vibrations due to its greater acceleration amplitude for any given voltage, than the ERM. 


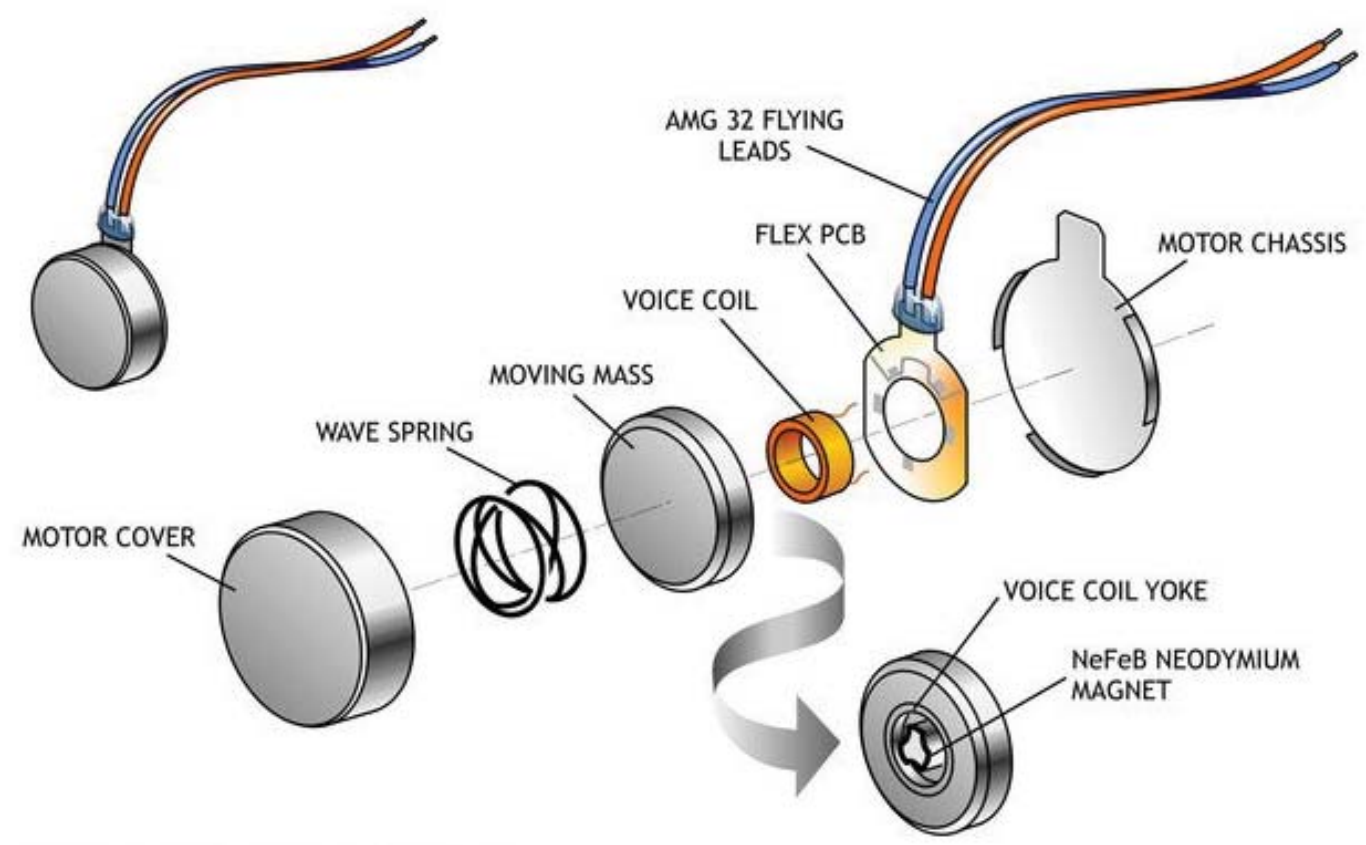

\section{PRECISION MICRODRIVES \\ PRECISION HAPTIC ${ }^{\text {TM }}$ Y-AXIS LINEAR RESONANT ACTUATOR}

Figure 3.7: Part breakdown of the LRA actuator Precision Microdrives web page

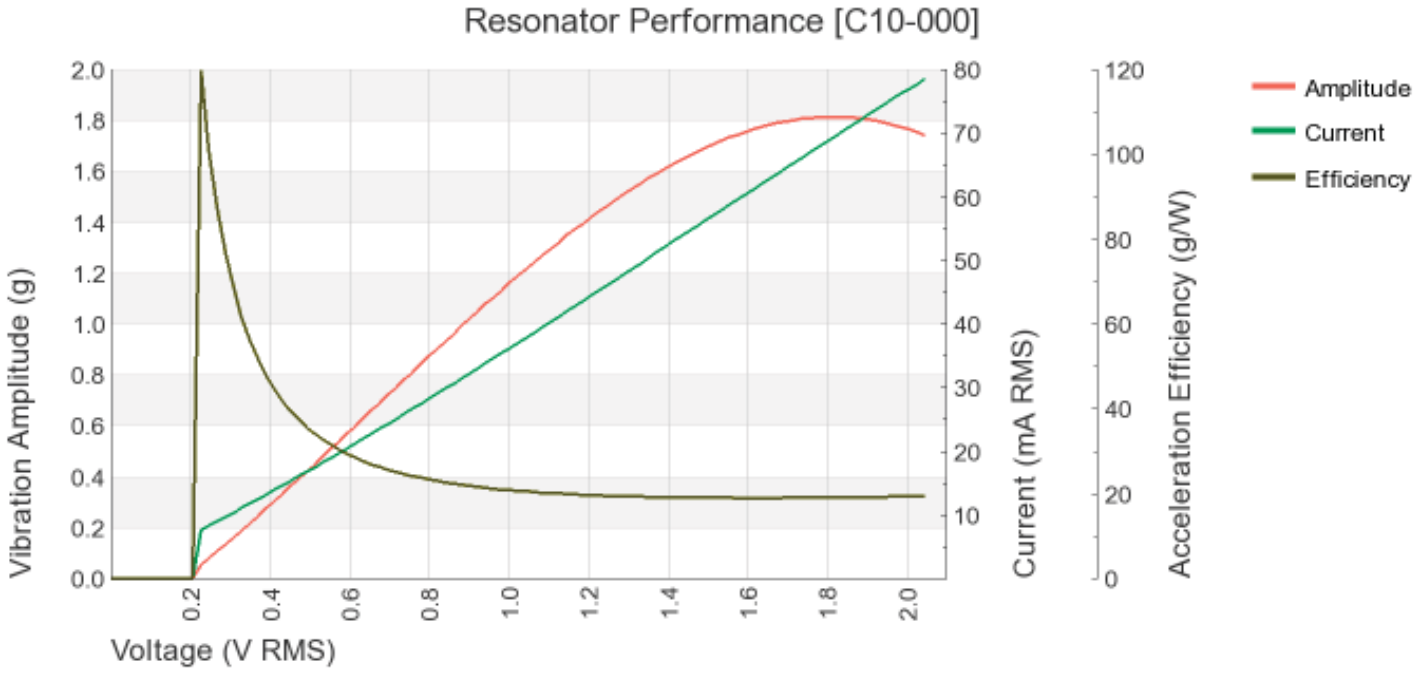

Figure 3.8: C10-000 tactor datasheet performance.

\subsubsection{Accelerometer (ADXL 345) Properties}

The ADXL 345, a tri-axial accelerometer was used in this study to measure the tactors' accelerations in the $\mathrm{x}, \mathrm{y}$ and $\mathrm{z}$. The ADXL 345, communicating with a MacBook 
Pro computer using the $\mathrm{I} 2 \mathrm{C}$ protocol, can sample at a frequency of approximately $1.6 \mathrm{kHz}$ (7 to 10 times that of both tactors' vibration frequencies), thereby removing aliasing artefacts and allowing for proper representation of the tactors' acceleration profiles.

\subsubsection{Tactor Powering}

PrecisionMicrodrives recommends using the TexasInstruments DRV 2603 chip to power LRAs since its auto-resonance tracking feature ensures that the LRA will always be driven by a signal matching its resonant frequency, regardless of the mounting surface [33]. In addition, the DRV 2603 can also be used to power DC motors like the ERM, thereby allowing for minimal changes in instrumentation between tests.

The DRV 2603 takes as input a PWM signal whose duty cycle controls the output voltage's amplitude and therefore the vibration amplitude for both the ERM and LRA [34]. The DRV 2603 has two modes; one to power an ERM for which it generates a DC voltage output, and one to power the LRA whereby it generates an AC voltage output with the same resonant frequency as that of the LRA. The DRV 2603's output voltage's amplitude or RMS value for each of the two modes can be calculated as shown in Equations 3.1 and 3.2.

$$
\begin{gathered}
V_{E R M}=3.6 \mathrm{~V} \cdot\left(\frac{P W M \text { Signal'sDutyCycle\% }}{50}-1\right) \\
V_{L R A(R M S)}=2.2 \mathrm{~V} \cdot\left(\frac{P W M \text { Signal'sDutyCycle\% }}{50}-1\right)
\end{gathered}
$$

In the above equations, the output voltage relationship shown for the ERM is true for all duty cycles, whereas the PWM signal duty cycles ranging from $0 \%$ to $50 \%$ all result in no vibrations for the LRA. Therefore a PWM signal duty cycle of $0 \%$ would cause ERMs to vibrate at the same maximum intensity observed for a duty cycle of 
$100 \%$, they would simply be vibrating in the opposite direction. In addition, a duty cycle of $50 \%$ would cause no ERM vibrations.

By using the DRV 2603 chip to power the tactors and the Arduino board to provide the PWM signal controlling the tactor's vibration amplitude, the current draw on the GPIO for the Arduino board (6 mA) is well within the $40 \mathrm{~mA}$ limit. In addition, the Arduino's Vcc pin can be used to power the DRV2603 pin which requires an operational current of up to $150 \mathrm{~mA}$ and voltage between 2.5 and $5.2 \mathrm{~V}$, well within the Arduino board's Vcc pin supply capabilities of $200 \mathrm{~mA}$ and $5 \mathrm{~V}$.

In order to provide a PWM signal, the analogWrite() function was used for the digital pins of interested which had a clock frequency of $31,372.55 \mathrm{~Hz}$, well above the DRV 2603's minimum required PWM frequency. The analogWrite() function can take in integer values ranging from 0 and 255, where 0 corresponds to a perpetually low PWM signal (duty cycle=0\%) and 255 corresponds to a consistently high PWM signal (duty cycle=100\%). The duty cycle of the PWM signal is then a ratio of 255 . For this reason a true $50 \%$ duty cycle cannot be achieved using the analogWrite() function; however, it was found that 127 and 128 could be used interchangeably to produce voltages of similar magnitudes that produce a PWM signal whose duty cycle is close enough to $50 \%$ to cause no vibrations in both the ERM and LRA. For this study, a $50 \%$ duty cycle was therefore provided using the analogWrite() function with a value of 128 .

For this experiment, the PWM signals provided for both tactor types had duty cycles within the same range $(128 / 255=50 \%$ to $248 / 255=97 \%)$ to allow for relative comparison but also to determine which tactor could be more effectively used with the power constraints associated with using the Arduino and DRV 2603 to power the motors. The voltages for the ERM and LRA are as shown in Table 3.3.

It can be seen from Table 3.3 that for the same PWM signal, the voltage across the ERM is much larger than across the LRA due to the differences in the DRV 2603's 
full scale output voltage for the ERM and LRA settings. To mitigate the effect of this difference in voltage on observed results, the plots are all with respect to the voltage across the tactor and not the voltage setting. Although this may increase the likelihood that ERM vibrations will be more noticeable, the LRA's vibration amplitude is shown to peak at $1.8 \mathrm{~V}$, and only further decreases as the voltage increases past this point. Therefore providing the LRA with a voltage range similar to that of the ERM would not increase the LRA's vibration amplitude or participants' ability to sense the LRA's vibrations (as seen in Figure 3.8).

Table 3.3: Arduino settings for each tested voltage and corresponding voltages across the ERM and LRA tactors

\begin{tabular}{cccc}
\hline Voltage Setting & $\begin{array}{c}\text { Arduino Duty } \\
\text { Cycle }\end{array}$ & $\begin{array}{c}\text { ERM Voltage } \\
\text { Across Tactor (V) }\end{array}$ & $\begin{array}{c}\text { LRA Voltage } \\
\text { Across Tactor (V) }\end{array}$ \\
\hline Voltage1 & $128 / 255=50 \%$ & 0 & 0 \\
Voltage2 & $148 / 255=58 \%$ & 0.53 & 0.35 \\
Voltage3 & $168 / 255=66 \%$ & 1.06 & 0.70 \\
Voltage4 & $188 / 255=74 \%$ & 1.58 & 1.04 \\
Voltage5 & $208 / 255=82 \%$ & 2.11 & 1.39 \\
Voltage6 & $228 / 255=89 \%$ & 2.57 & 1.72 \\
Voltage7 & $248 / 255=97 \%$ & 3.10 & 2.07 \\
\hline
\end{tabular}

\subsubsection{Attachment to Body Sites}

In order to attach the tactors to the various body locations, MediCare Heavy Fabric Adhesive Bandages were used since their non-adhesive pads decreased the damping effect on the vibration amplitude and allowed more side-to-side motion than would be seen if the tactor were connected directly to an adhesive. This is of great importance for the ERM since its main motion direction is parallel to the bandage and would be more greatly restrained by an adhesive on the restraining bandage than by the 
bandage's pressure exerted. Inversely, having an adhesive surface would have a smaller effect on the vibration intensity of the LRA which vibrates perpendicularly to the skin and receives more resistance to motion from the tension in the bandage than it would from being attached to the bandage.

A new bandage was used for each test and fixed to the participant's skin with an inherent tension of 2N, measured using a dynamometer (Equation 3.3 shows how the $2 \mathrm{~N}$ tension can be converted to the mass units shown on the dynamometer) as illustrated in Figure 3.9. By maintaining the same bandage tension between tests, sub-bandage pressures are consistent across all tests [35]. This is important, since this pressure offers resistance to tactor motion thereby decreasing vibration amplitude; a tension of $2 \mathrm{~N}$ was found trough trial and error to keep the tactor/accelerometer setup securely in place while also allowing for highly noticeable tactor motion.

$$
\text { mass }=\frac{2 N}{9.81 \frac{\mathrm{m}}{\mathrm{s}^{2}}}=0.204 \mathrm{~kg}
$$

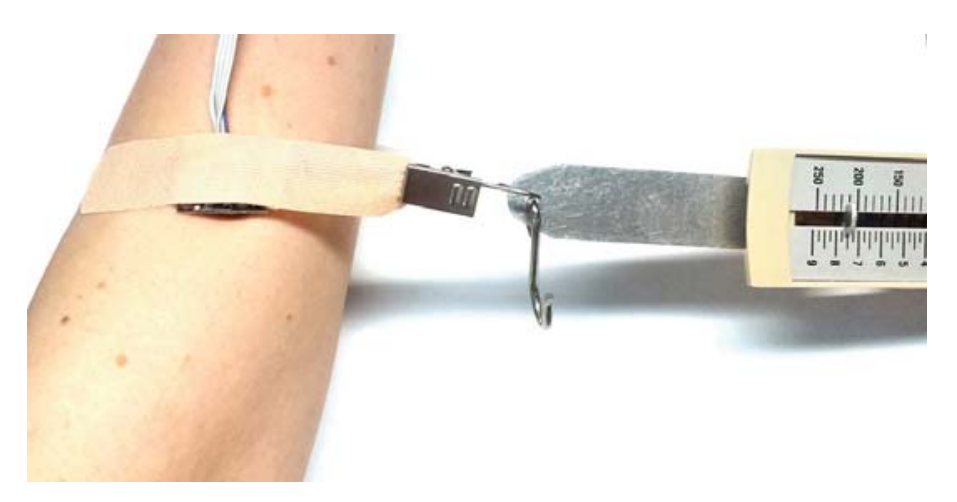

Figure 3.9: A dynamometer is used to ensure constant bandage pressures when attaching tactor/accelerometer setup to participants' bodies. 


\subsubsection{Body Locations Tested}

Three body tissue types (muscular, bony and lipid) representative of the range of body tissue types onto which tactors will be mounted in future studies were tested in this study (Figure 3.10 generally depicts the body locations tested as part of this study). This was done since the tactor type chosen for future feedback location optimization studies should be easily sensed over all body locations tested.

Muscle tissue testing was conducted over the flexor carpi ulnaris (forearm) chosen for its ease of accessibility and low levels of adipose tissue across participants.

Bony tissue testing was conducted over the patella since it is a large flat bony section which allows for proper tactor attachment and because of its ease of accessibility.

Lastly, lipid tissue testing was conducted 1 inch distally to the umbilicus (abdomen) because it is the most easily accessible location often high in adipose tissue and tactor attachment could easily be achieved on this site.

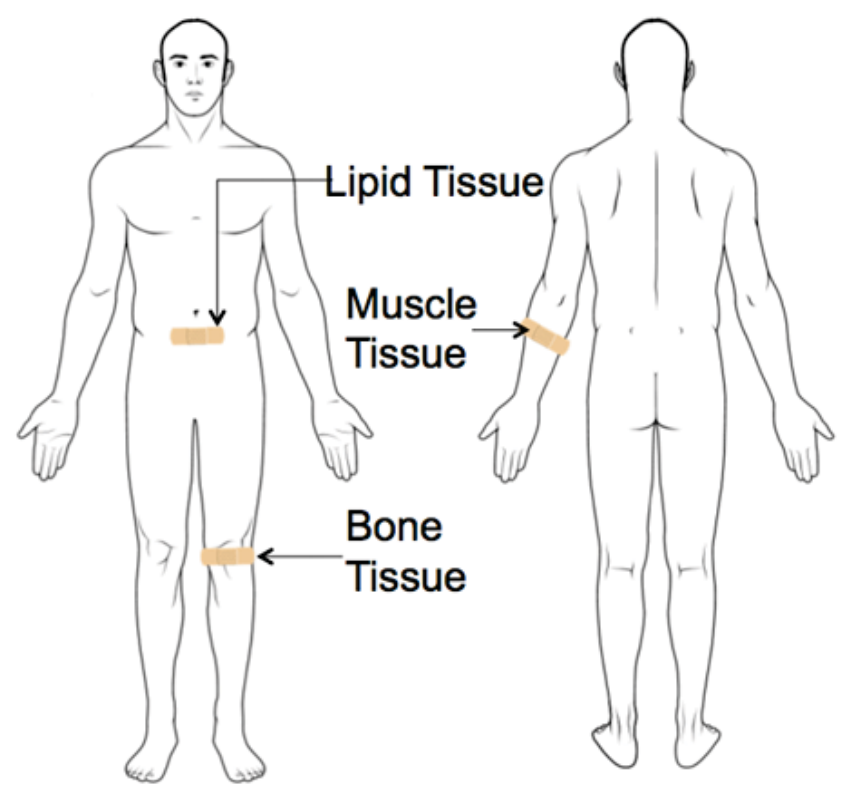

Figure 3.10: Body locations tested as part of the study, with biomaterial type indicated. 


\subsubsection{Measures of Comparison}

To characterize tactor vibrations and act as predictor measures, the maximum Power Spectral Density (PSD), vibration frequency, acceleration amplitude and displacement amplitude were calculated. The PSD and vibration frequency are found through PSD analysis of the tactors' acceleration data, where the signal's dominant frequency (frequency that exhibits the maximum PSD when spectral analysis is performed on the signal data) is identified as the vibration frequency. By determining the Fourier equation representative of the acceleration profile of each tactor in each direction, the acceleration amplitude can be found. Furthermore, through double integration of this Fourier equation, the displacement amplitude can be calculated, as will be shown later in this chapter. Moreover, to allow for the determination of which tactor type should be included in future studies, the percentage of cases where participants detected feedback for each voltage tested was calculated for each tactor type.

\subsubsection{Data Analysis}

Data analysis was performed through post-processing using MATLAB software (Release 2014b). Using this software, the Fourier representations for both the tactor acceleration and displacement were developed based on the measured tactor accelerations. MATLAB was also used to calculate the max PSD, vibration frequency, acceleration amplitude and displacement using the recorded acceleration data for each tactor, at each voltage tested.

To determine which tactor type should be used in further studies, the percentage times feedback was detected for each voltage, regardless of the tactor location, was calculated for each tactor type.

In order to determine the effectiveness of each measure in predicting participants' ability to detect feedback, the sensitivity and specificity of each predictor measure 
were calculated. This was done by comparing the outcomes predicted using each predictor measure to participants' verbal identification of their ability to detect feedback. In order to calculate these, the number of true positive, false positive, true negative and false negative instances had to be calculated based on the definitions outlined in Figure 3.11.

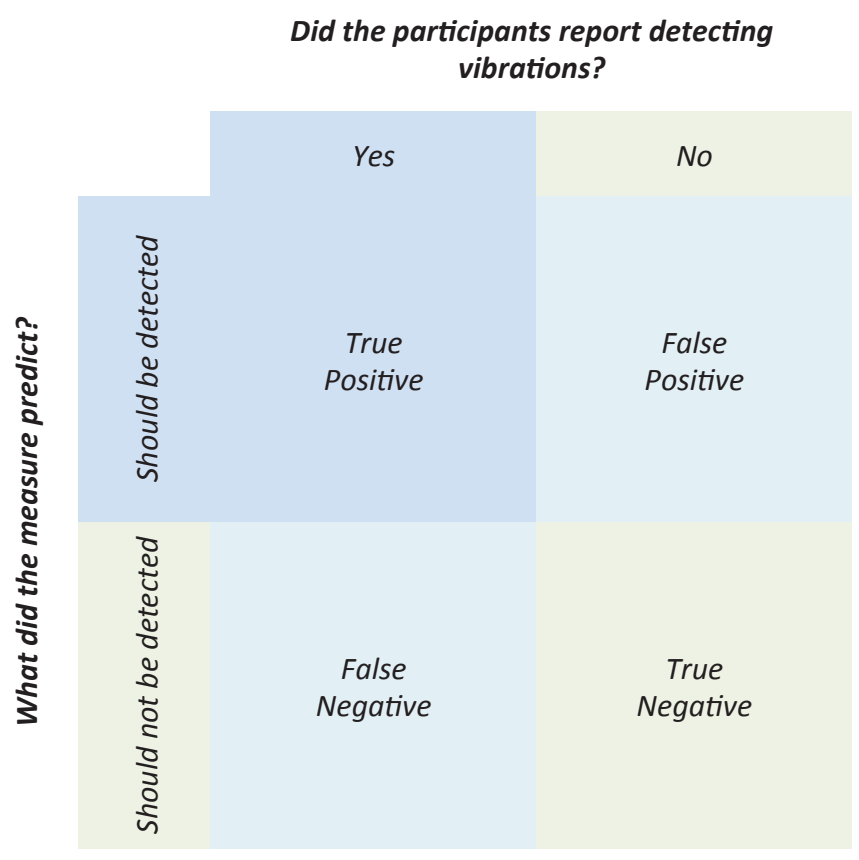

Figure 3.11: Meaning of true positive, false positive, true negative, and false negative.

The sensitivity is the probability, ranging from 0 to 1 , that the predictor value would indicate a participant having detected feedback when in actuality the participant reported detecting feedback, calculated as shown in Equation 3.4 [36]. This in effect is the percentage of instances where participants reported detecting feedback that were correctly predicted using the predictor measure.

$$
\text { Sensitivity }=\frac{\text { TruePositive }}{\text { TruePositive }+ \text { FalseNegative }}
$$

And specificity is the probability, ranging from 0 to 1 , that the predictor value 
would indicate no feedback detected when the participant in actuality did not detect feedback, calculated as shown in Equation 3.5 [36]. This in effect is the percentage of instances where participants reported not detecting feedback that were correctly predicted using the predictor measure.

$$
\text { Specificity }=\frac{\text { TrueNegative }}{\text { TrueNegative }+ \text { FalsePositive }}
$$

The sum of the sensitivity and specificity corresponds to the percentage of correct predictions made on a participant's ability to detect feedback. The maximization of this sum is an effective method in classifying the usefulness of a predictor measure in cases like this one, where the consequence associated with incorrectly predicting a no-detection event and of incorrectly predicting a detection event are the same [37].

\subsection{Results and Discussion}

\subsubsection{Data Filtering}

In order to remove the noise effects associated not only with the circuit used to power the tactors, but also with natural human motions from the analyzed data, the acceleration data was filtered. Filtering the data also smoothed the acceleration signal considerably since the sampling frequency, although 7-10 times that of the tactors' vibration frequency, caused some jagged trends in the measured data.

A 7th order Butterworth bandpass filter was used to filter the data. The 7th order filter was chosen because it offered the sharpest roll-ff without presenting any instability near the cutoff frequency.

Different high-pass cutoff frequencies were used to filter the ERM and LRA data since, according to their datasheet, their vibration frequencies are vastly different. According to its datasheet, the ERM vibrates at frequencies ranging from 50 to 180 
$\mathrm{Hz}$, however experimentally these frequencies ranged only up to $130 \mathrm{~Hz}$. Therefore a high-pass cutoff frequency of $180 \mathrm{~Hz}$ was chosen for the ERM signal, thereby removing unwanted high-frequency noise while ensuring that none of the vibration's higher frequency behavior would be filtered out.

The LRA's vibration frequency, although dependent on the mounting surface, was found through preliminary experiments to range up to about $250 \mathrm{~Hz}$. Therefore the LRA's high-pass cutoff frequency was chosen as $310 \mathrm{~Hz}$ to account for interparticipant variations in vibration frequency. This ensured that even if the vibration frequency was to increase for this study's participants, the higher frequency content corresponding to the tactor's vibrations would not be filtered out.

The low-pass cutoff frequency was the same for both tactor types and was set as $15 \mathrm{~Hz}$ to remove human motion and gravity effects. Since voluntary human motions have lower frequencies than that of low-frequency tremors which occur at a frequency of $4 \mathrm{~Hz}$ [38] [39], a cutoff value more than 3 times this value is considered adequate at removing human motion effects from the analyzed data.

\subsubsection{Filter Effect on Circuit's Inherent Noise}

Figures $3.12 \mathrm{a}$ and $3.12 \mathrm{~b}$ show the PSD values obtained when analyzing the acceleration profile for both an ERM and LRA tactor connected to the powered circuit but not undergoing vibrations. This illustrates the noise associated with the tactor powering and accelerometer communication circuit alone. It can be seen from both plots that the circuit causes considerable high frequency noise, especially in the ERM signal which exhibits noise magnitudes of over 500 times that of the LRA signal. Although this high frequency noise is removed using the filter, if the ERM is selected for use in future tests, another tactor driving circuit should be developed.

The circuit connection noise is of interest since, even though it is predominantly present in the high frequency range near $400 \mathrm{~Hz}$, there is still noise resulting from 
connecting the tactors to the circuit present within the bandpass frequency range. In the case of the ERM, the maximum PDS observed for the filtered signal is of less than 5, which is much smaller than PSD peaks observed for instances where tactors are vibrating which typically exhibit magnitudes ranging from 20 to 3000 for all voltage ranges tested (see Appendix C). Therefore this noise would bear no effect on the identification of the ERM's vibration frequency.

In contrast, Figure 3.12b shows that even for the LRA's filtered signal, noise is found in the frequency range of interest $(15-310 \mathrm{~Hz})$. In fact, the noise's PSD magnitude in the frequency range of interest can reach up to 0.005, which is still at least 10 times smaller than typical LRA PSD peaks which range from 0.05 to 300 for all voltages tested (see Appendix C).

It is however possible that the circuit's noise effects could impact the results observed; however, this is only likely to occur in instances where the voltage across the tactor is very low, or there is almost no motion in the direction of interest.
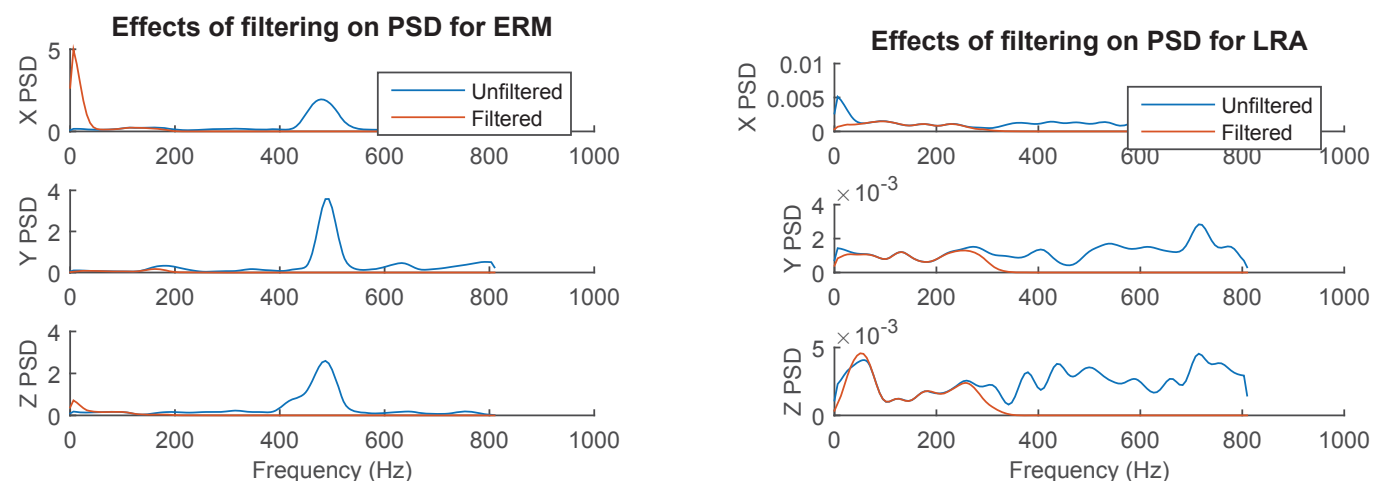

(a)

(b)

Figure 3.12: Effects of filtering the acceleration signal when the circuit is powered but no voltage is applied across the tactor.

\subsubsection{Filter Effect on Motion Artifacts}

In order to test the efficacy of the bandpass filtering in removing noise caused by human motion, two tests (one with the LRA and one with the ERM) were conducted 
with a tactor mounted on a test subject's forearm. The subject would swing his/her arms as quickly as possible while the tactor vibrated at each of the 7 voltages tested as part of this study were used to power the tactor. The acceleration measured by the accelerometer placed on the tactor was recorded for each of voltages and analyzed to determine the effects of human motion on the recorded and filtered data.

Figure 3.13 displays this test's unfiltered and filtered (using the filters mentioned in the previous section) acceleration signals for both the ERM and LRA. As can be seen in Figure 3.13a, the majority of the signal's power is associated with the ERM's vibration for Voltage 4 and upwards. However, at lower voltage levels, the unfiltered signal's PSD spectrum's peak shifts from the tactor's vibration frequency to much lower frequencies corresponding to the arm swing's frequency. Figure 3.13c shows this noise's effect on the the ERM's PSD peak location is removed when bandpass filtering the signal data.

In contrast, Figure $3.13 \mathrm{~b}$ shows that in the unfiltered signal the arm motion's PSD and influence on the recorded signal is much greater than that associated with the LRA's vibration in the $\mathrm{x}$ and $\mathrm{y}$ directions, but not in the tactor's main vibration direction (z direction). Although filtering the signal attenuates the PSD associated with the swinging arm motion, it only reduces it to a magnitude similar to the PSD associated with the LRA's vibration, as seen in Figure 3.13b. This however should not cause large discrepancies in the study's data analysis since no motions of this magnitude will be executed by participants who are asked to remain as still as possible for this study's tests. 


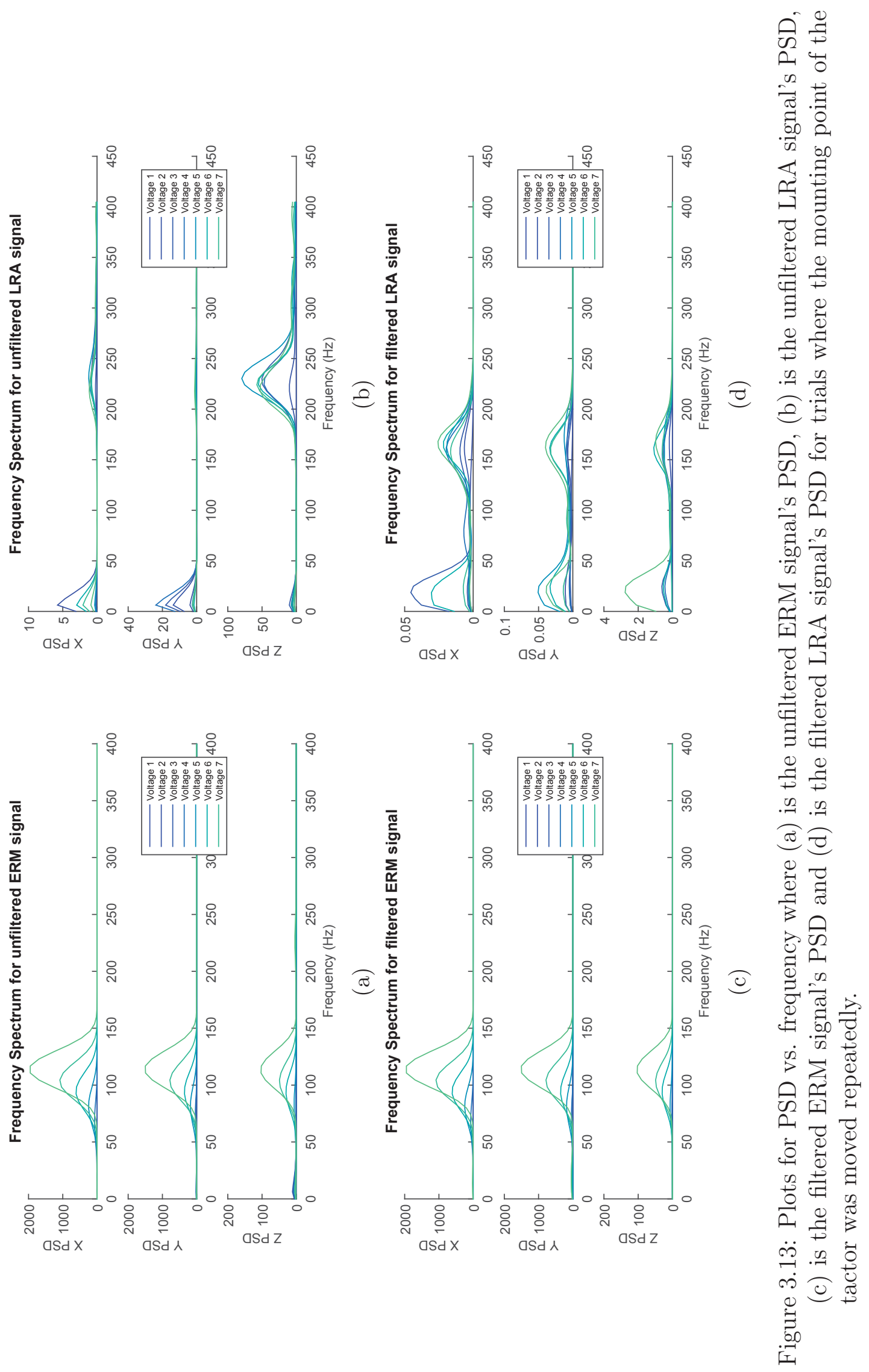




\subsubsection{Acceleration and Displacement Amplitude Calculation}

According to both tactors' datasheets, each tactor type is supposed to vibrate at a fixed frequency for each voltage at which it is powered, which would allow for easy modeling of the system dynamics and calculation of the acceleration and displacement amplitudes. However, through experimentation (and as as shown Figure 3.13), it was found that the peak in the PSD spectrum does not only span a single frequency, but rather a narrow band of frequencies that exhibit PSDs much greater than other frequencies outside the band. This indicates that the tactors' vibration waveforms can best be represented using a combination of sinusoidal waveforms rather than a single sinusoidal waveform.

In order to determine how many terms should be used to represent the measured accelerations, Fourier equations with 1, 2, 4, 6 and 8 sinusoidal terms were used to represent the data and their accuracy in reconstructing the acceleration signal was compared. It was found that the mean residuals (differences at each time point between the recorded and reconstructed acceleration) did not vary meaningfully with the number of terms used in the Fourier equation. In fact, on average, a single sinusoidal term accounted for about $90 \%$ of the reconstructed waveform's amplitude whereas the other additional terms only cumulatively accounted for $10 \%$ of the waveform amplitude. For these reasons, the assumption that the tactor's acceleration profile could be represented using a single sinusoidal term was considered valid. Therefore the acceleration can be reconstructed using an equation the form:

$$
a=A \sin (\omega t)
$$

Where $a$ is the acceleration, $A$ is the acceleration amplitude and $\omega$ is the vibration frequency found through PSD analysis (the frequency that corresponds to the maximum PSD is considered to the be the tactor's vibration frequency). We know 
that the acceleration is the derivative of the velocity, thus the velocity can be found as:

$$
v=-\frac{A}{\omega} \cos (\omega t)
$$

Where $v$ is the tactor velocity. Similarly, the displacement can be found by integrating once again to be:

$$
d=-\frac{A}{\omega^{2}} \sin (\omega t)=-D \sin (\omega t)=-\frac{a}{\omega^{2}}
$$

Therefore the displacement amplitude $D$ can be related to the acceleration amplitude $A$ using the vibration frequency as being:

$$
D=\frac{A}{\omega^{2}}
$$

As aforementioned, the dominant vibration frequency can be found using the power spectral density analysis results. The acceleration amplitude can be calculated using the acceleration data's RMS value, because the following relationship exists:

$$
a_{R M S}=\frac{A}{\sqrt{2}}
$$

Therefore the displacement amplitude can be found as:

$$
D=\frac{a_{R M S} \sqrt{2}}{\omega^{2}}
$$

\subsubsection{Vibration Characteristics}

This section helps provide a more complete understanding of the ways in which the ERM and LRA operate when placed on various body sites. 


\subsubsection{Maximum PSD}

Maximum PSD for ERM on Lipid, Muscle and Bone (Left to Right)

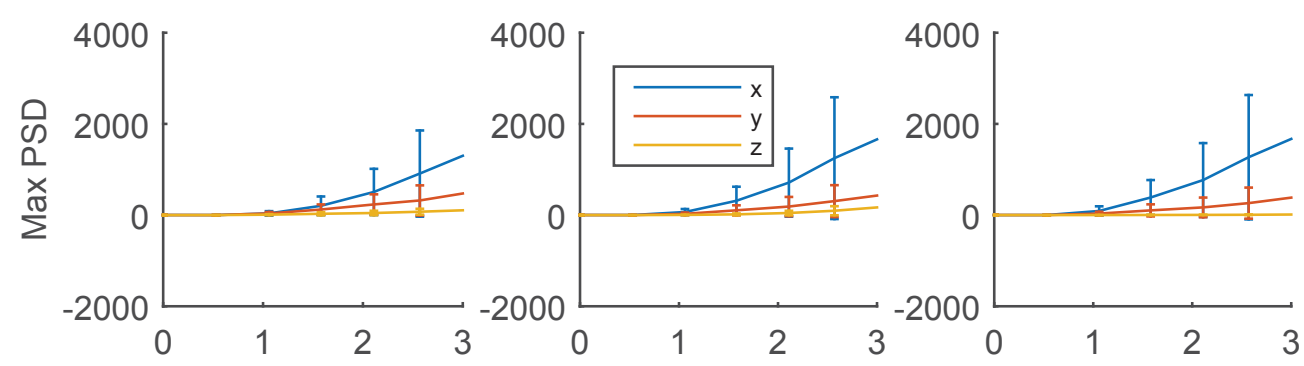

Maximum PSD for LRA on Lipid, Muscle and Bone (Left to Right)
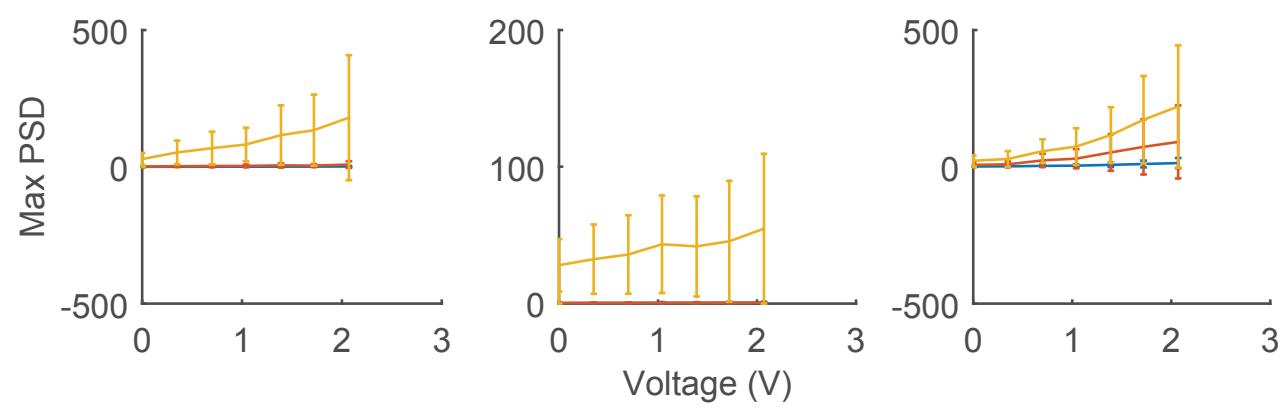

Figure 3.14: Plots of maximum PSD for vibration in the $\mathrm{x}, \mathrm{y}$ and $\mathrm{z}$ directions for each tactor type on each biomaterial tested.

In Figure 3.14 it can be observed for both tactor types that the maximum PSD increased as a function of the voltage supplied to the tactor and that PSDs were greater for the tactors' predominant vibration direction(s) (x and y directions for the ERM and $\mathrm{z}$ direction for the LRA). This is due to the fact that the max PSD is actually the PSD associated with the tactors' vibration frequency; for greater vibration amplitudes (which are observed at greater supply voltages or in the dominant vibration directions) the proportion of the signal corresponding to these vibrations increases, causing the PSD at the vibration frequency, and therefore the max PSD, to increase. Similarly, the ERM's PSD is much greater than the LRA's, which is also likely due to the fact that the vibration amplitude of the ERM is greater than the LRA's. Lastly, there are no noticeable inter-tissue type differences in the max PSD 
recorded.

With regards to using the maximum PSD as a predictor for the wearer's ability to sense biofeedback, the inter-participant and inter-test variations are so large that this data would prove useless as a predictor.

\subsubsection{Vibration Frequency}

Frequency for ERM on Lipid, Muscle and Bone (Left to Right)
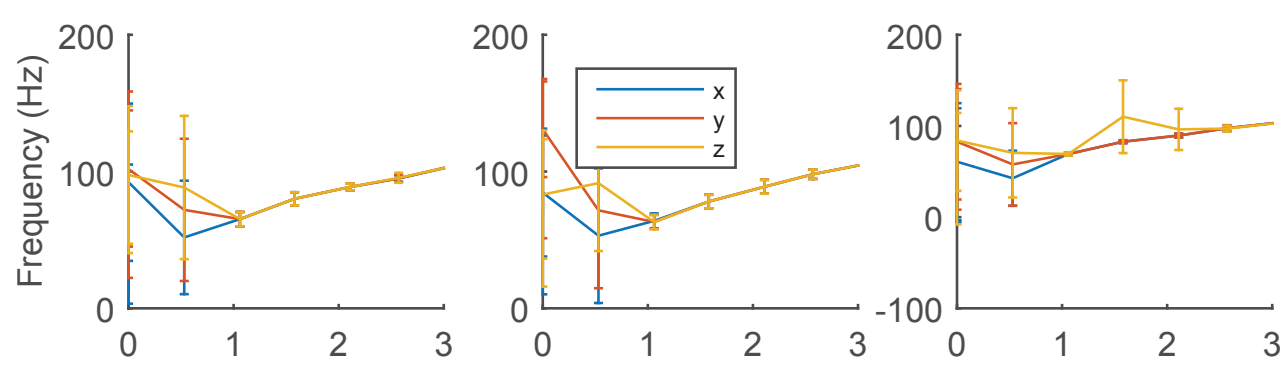

Frequency for LRA on Lipid, Muscle and Bone (Left to Right)
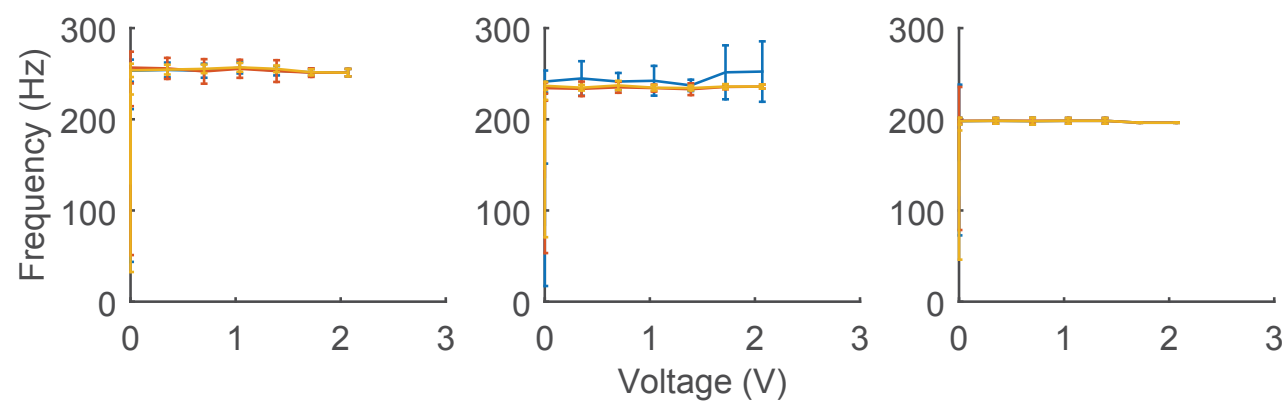

Figure 3.15: Plots of tactor vibration frequency in the $\mathrm{x}, \mathrm{y}$ and $\mathrm{z}$ directions for each tactor type on each biomaterial tested.

Figure 3.15 demonstrates, as outlined in the datasheets, that the ERM's vibration frequency varies with the supply voltage and that the LRA's vibration frequency depends on the tissue type rather than the supply voltage. Furthermore, the vibration frequency of the LRA (200-250 Hz) is much larger than the ERM's vibration frequency (75-125 Hz), behaving as depicted in the datasheets. This could have implications on the participants' ability to sense feedback since both tactor types would trigger different mechanoreceptors as outlined in a later section. 
It can be observed that the vibration frequency of the LRA decreases slightly as the mounting tissue stiffness increases. However, these differences prove small when considering the vast differences in stiffness between lipid, muscle and bone tissue; this could indicate that the epithelial layers between the LRA and the lipid, muscle or bone have a more significant impact on the associated resonant frequency of the LRA-tissue setup than the underlying tissue.

Figure 3.15 also shows large variations in vibration frequency for the ERM at low voltages. This is likely due to the fact that these lower voltages ( 0 and $0.53 \mathrm{~V})$ are either below or very close to the ERM's start-up voltage; this could cause the tactor to sporadically vary between turning on and off which would lead to random non-sinusoidal acceleration profiles and large variations in vibration frequencies.

\subsubsection{Acceleration Amplitude}

\section{Peak Acceleration for ERM on Lipid, Muscle and Bone (Left to Right)}
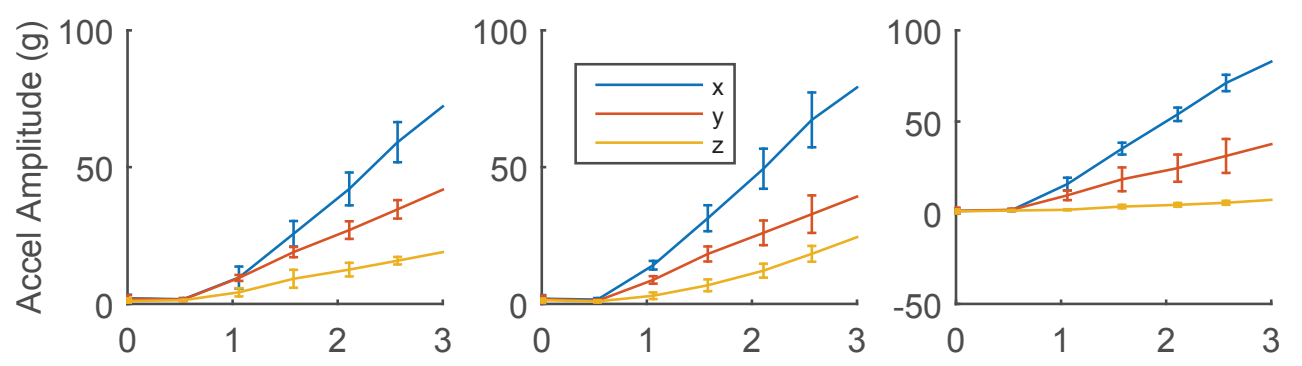

Peak Acceleration for LRA on Lipid, Muscle and Bone (Left to Right)
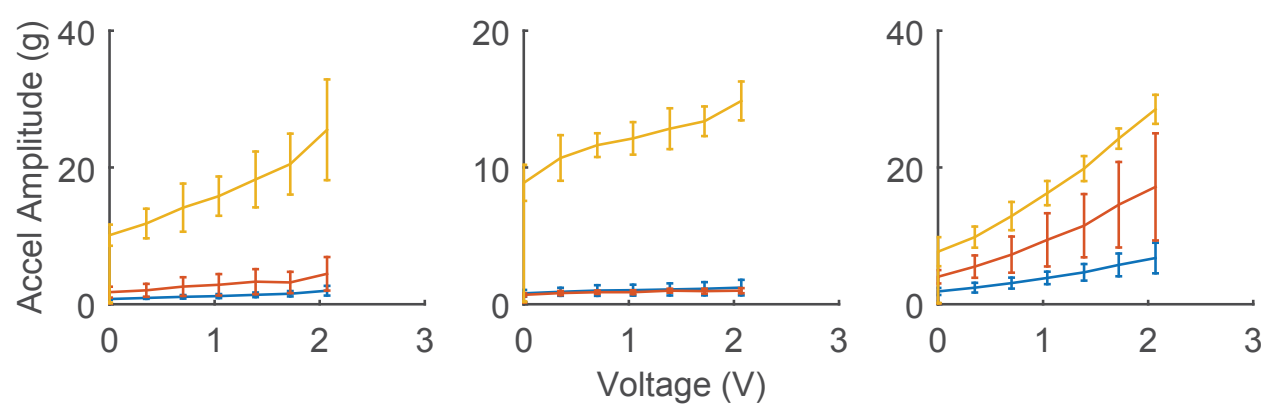

Figure 3.16: Plots of acceleration amplitude in the $\mathrm{x}, \mathrm{y}$ and $\mathrm{z}$ directions for each tactor type on each biomaterial tested. 
As shown in Figure 3.16 both the ERM and LRA experience increasing acceleration amplitudes as the voltage increases and the predominant vibration directions are in accordance with the actuation system used for each tactor. For instance, the $\mathrm{x}$ and $\mathrm{y}$ acceleration amplitudes are greater than the $\mathrm{z}$ amplitude for the ERM indicating primary motion parallel to the user's skin surface. Inversely, the z acceleration amplitude is greater than its $\mathrm{x}$ and $\mathrm{y}$ counterparts for the LRA, which indicates that its motion is primarily perpendicular to the skin's surface.

What is somewhat perplexing is that the ERM's acceleration amplitude in the $\mathrm{x}$ direction is greater than in the y direction, which is unexpected since the ERM should be providing the same acceleration amplitude in the $\mathrm{x}$ and $\mathrm{y}$ directions. This could be due to the fact that the accelerometer chip was not aligned with the ERM's vibration axis and would mean that only the measured acceleration profile is skewed, but that in reality the $\mathrm{x}$ and $\mathrm{y}$ acceleration amplitudes are the same. The accelerometer chip's axis was offset from the tactor's axis in both the $\mathrm{x}$ and $\mathrm{y}$ directions; the offset was greater in the $\mathrm{x}$ direction than it was in the $\mathrm{y}$ direction. Since the ERM's vibrations are about its axis, this causes tangential and centripetal accelerations to be recorded by the accelerometer in the $\mathrm{x}$ and $\mathrm{y}$ directions. Since these two accelerations produce linear accelerations proportional to the radius from the center of rotation, it holds that the accelerometer would measure greater accelerations in the $\mathrm{x}$ directions because of the increased distance.

Also unexpected was the fact that, for equivalent voltages, the ERM vibrated with a greater vibration amplitude than the LRA, which contradicts the relationship outlined in the tactors' datasheets. The difference between the results observed in this study and the datasheet values may be due to differences between the setups used by Precision Microdrives and this study. Precision Microdrives's experimental set-up, shown in Figure 3.17, consisted of a tactor attached rigidly to a $100 \mathrm{~g}$ freemoving mass made of a uniform metal suspended by silicon bands onto which an 
accelerometer and PCB are rigidly mounted to measure the vibration strength and frequency as well as supply the motor with power [40]. However in haptic applications like this study, tactors are typically attached elastically to a fixed mass with nonuniform mechanical properties (e.g. skin). The resonant frequency for the LRA on a uniform metal would be more consistent and easier to identify than when the LRA is on a complex surface like skin-covered lipid/muscle/bone. Therefore the powering frequency of the LRA would be much closer to the resonant frequency and therefore in the optimal powering range for the Precision Microdrives setup which would cause greater vibration amplitudes than for this experiment's setup. This seems to indicate that the LRAs are not well suited to providing vibrotactile feedback in haptic applications.

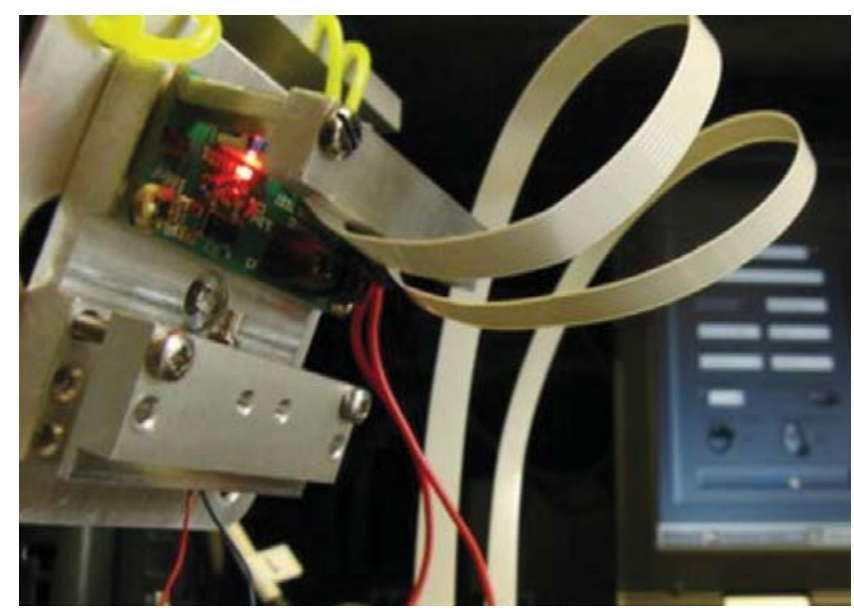

Figure 3.17: Experimental set-up used by Precision Microdrives to determine vibration frequency, acceleration amplitude and efficiency as a function of voltage.

\subsubsection{Displacement Amplitude}

In Figure 3.18 we see behaviors very similar to those observed in Figure 3.16. Once again, the displacement amplitudes are greater for the ERM than the LRA, and are also greater in the predominant vibration direction as previously explained. Although no new trend or phenomenon is shown using this data, it may still act as a useful predictor of peoples' ability to sense feedback. 


\section{Displacement for ERM on Lipid, Muscle and Bone (Left to Right)}
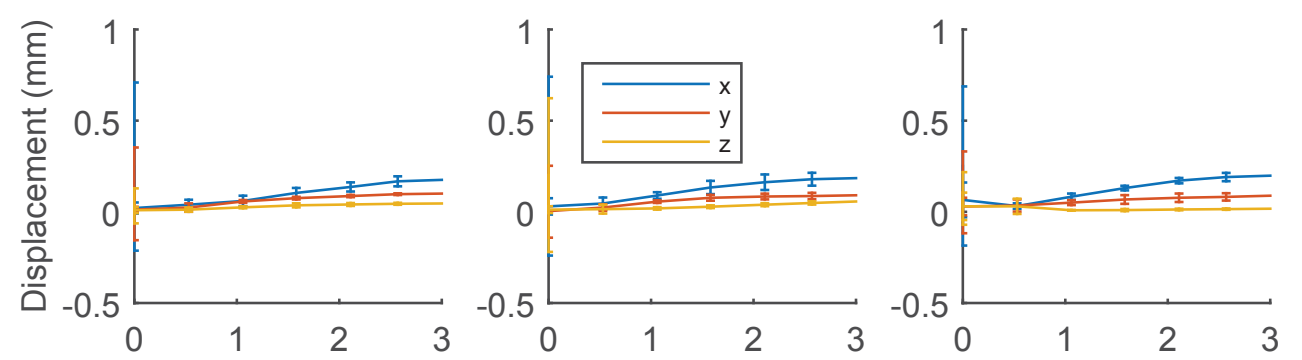

Displacement for LRA on Lipid, Muscle and Bone (Left to Right)
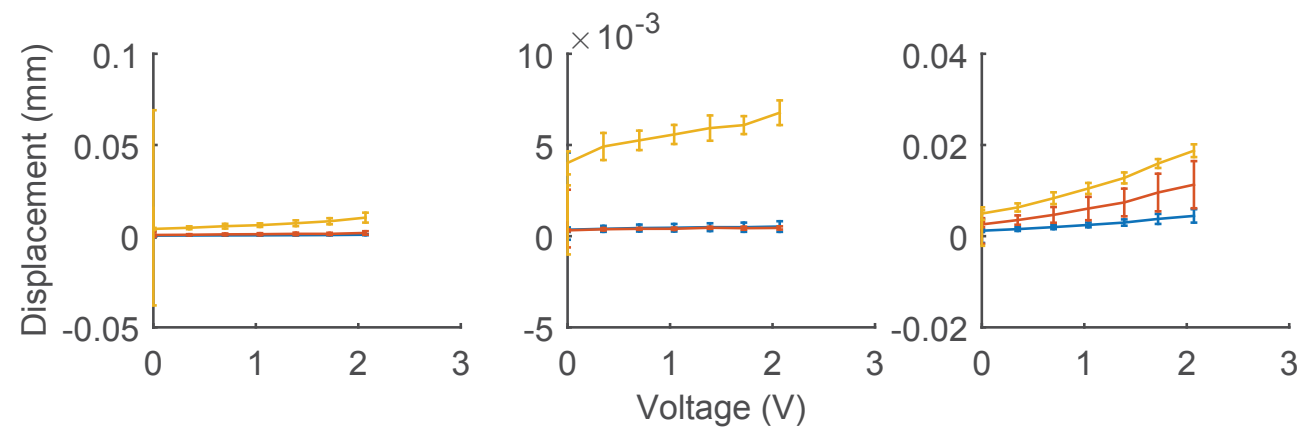

Figure 3.18: Plots of tactor vibration displacement amplitude in the $\mathrm{x}, \mathrm{y}$ and $\mathrm{z}$ directions for each tactor type on each type of biomaterial tested.

\subsubsection{Mechanisms of Vibration Sensing for Both Tactor Types}

The LRA, with its vibration frequency range of $200-250 \mathrm{~Hz}$, would only trigger the Pacinian corpuscles since its vibration frequency is outside the operational frequency range of other mechanoreceptors [27]. Although the LRA's vibration frequency is within the optimal range of frequencies for detection using the Pacinian corpuscles, the displacement amplitude of the LRA must be greater than $0.02 \mathrm{~mm}$ in order to trigger the Pacinian corpuscles [28]. It can be seen from Figure 3.18 that the LRA's displacement amplitudes in most cases fall short of $0.02 \mathrm{~mm}$ and therefore are likely not to be sensed by the Pacinian corpuscles, which would explain why the LRA vibrations were often not felt by participants.

The ERM, with its vibration frequency range of 75-125 Hz, would trigger both the Pacinian corpuscle and the Meissner's corpuscles since its vibration frequency 
is within the operational range of both mechanoreceptors. Although the ERM's vibration frequency is not in the Pacinian corpuscles' optimal range of operation, the Pacinian corpuscles can still sense ERM vibrations provided the displacement amplitude is large enough [28]. In fact, Figure 3.2 shows that for vibration frequencies of $75 \mathrm{~Hz}$, a vibration displacement of $0.1 \mathrm{~mm}$ or greater would be detected by the Pacinian corpuscles; and for vibration frequencies of $125 \mathrm{~Hz}$, a vibration displacement of $0.05 \mathrm{~mm}$ or greater would be detected. These values are the sensing thresholds for vibrations perpendicular to the skin, but in the case of vibrations parallel to the skin, as is the case with the ERM, these thresholds are effectively lowered [28]. The ERM's displacement amplitudes as shown in Figure 3.18 for the most part exceed the thresholds associated with vibrations perpendicular to the skin, which would explain why ERM vibrations were always sensed by participants. Furthermore, the Meissner's corpuscles would also sense the ERM vibrations [27], and since they are the most common mechanoreceptor found in glaborous skin [26], the ERM's vibrations are liable to trigger more mechanoreceptors than the LRA's vibrations further supporting the fact that ERM vibrations were more easily sensed than LRA vibrations.

\subsubsection{Tactor Selection for Future Studies}

Overall qualitative feedback from participants expressed a clear and unanimous increase in ability to detect the feedback provided by the ERM. This opinion is reflected in the instances of detected feedback for both tactor types reported in Table 3.4 where feedback provided using the ERM was felt at all instances where a non-zero voltage was applied to the ERM. In comparison, at the lowest non-zero voltage, the LRA was only sensed $56 \%$ of the time by participants, and even at higher voltages, it was only felt $89 \%$ of the time.

This increased performance in a tactor that vibrates at a frequency ranging from 
75-125 Hz, more than two times smaller than the commonly used vibrotactile feedback frequency of $250 \mathrm{~Hz}$ [18] [41] [42] was in fact very unexpected. It goes to show that existing studies that only consider a tactor's vibration frequency when selecting tactors are potentially making poor decisions, because the type of tactor actuation matters much more than the vibration frequency when trying to improve user vibration detection. In fact this study supports another study's statement that vibrations parallel to the skin's surface are more easily sensed than vibrations perpendicular to it $[28]$.

Based on user feedback and the recorded likelihood of sensing feedback using both types of tactors, the ERMs were chosen as the best tactor type for feedback, a result that mirrors the observations found when characterizing tactor vibration properties as well. In order to provide useful feedback in future studies, the ERMs should be powered using a voltage of $0.53 \mathrm{~V}$ or greater to ensure that they are felt by participants at all times.

Table 3.4: Percentage times feedback was felt for each tactor type at each voltage setting

\begin{tabular}{cccc}
\hline & ERM & \multicolumn{2}{c}{ LRA } \\
Voltage $(\mathrm{V})$ & $\begin{array}{c}\text { \% detected } \\
\text { feedback }\end{array}$ & Voltage $(\mathrm{V})$ & $\begin{array}{c}\text { \% detected } \\
\text { feedback }\end{array}$ \\
\hline 0 & 0 & 0 & 0 \\
0.53 & 100 & 0.35 & 56 \\
1.06 & 100 & 0.70 & 89 \\
1.58 & 100 & 1.04 & 89 \\
2.11 & 100 & 1.39 & 89 \\
2.57 & 100 & 1.72 & 89 \\
3.10 & 100 & 2.07 & 89 \\
\hline
\end{tabular}




\subsubsection{Vibration Detection Prediction}

\subsubsection{Specificity and Sensitivity}

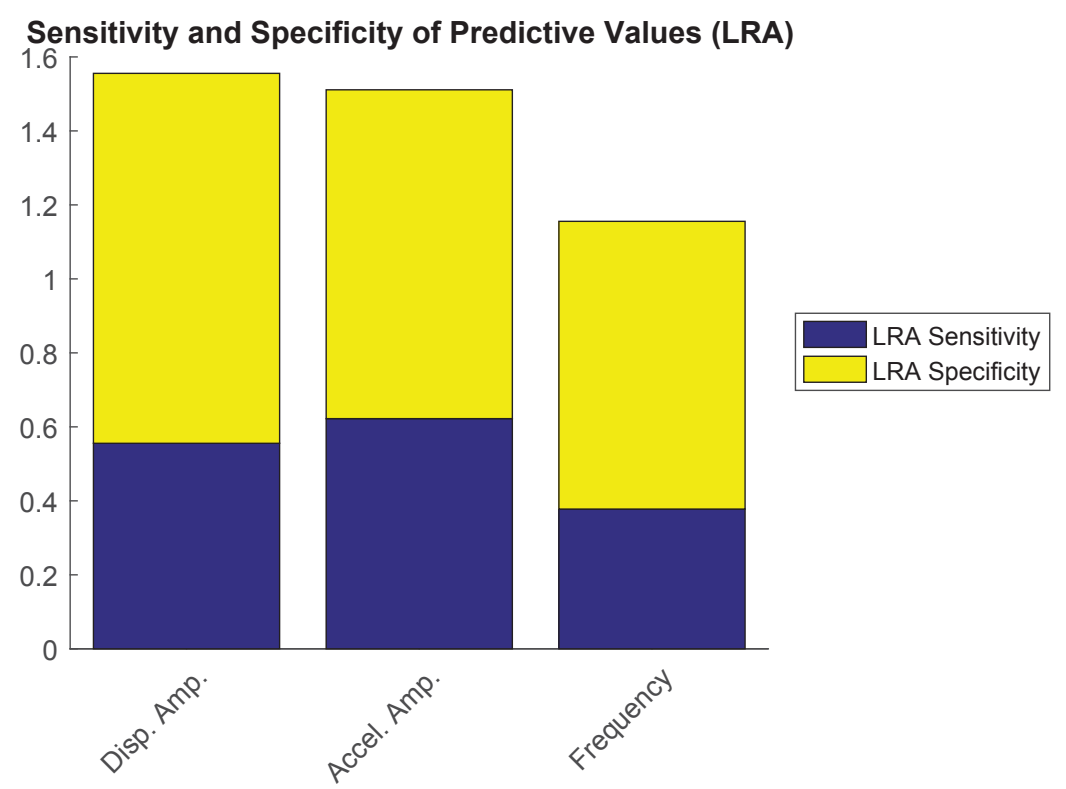

Figure 3.19: Specificity and sensitivity associated with each metric for the LRA.

Results for LRA Data: Figure 3.19 demonstrates the ability to predict whether or not the wearer will perceive the feedback provided by the tactor. The nominal displacement amplitude, acceleration amplitude and frequency in the predominant motion direction were used as potential predictors to be evaluated. By taking the height of each bar and dividing it by 2 we can find the percentage of correct predictions made using each predictor; therefore a predictor capacity to correctly identify the wearer's ability to sense feedback is proportional to the height of its data column. The greater the sum of the sensitivity and specificity (and therefore the column), the more useful a predictor measure [37].

The vibration frequency is the worst predictor of a person's ability to sense feedback, predicting correctly if participants would detect feedback less than $58 \%$ of the time. This is likely due to the fact that the LRA's vibration frequency is consistent 
for all voltages, and therefore cannot be used to identify differences in feedback intensities provided to the user. Furthermore, LRA feedback cannot be detected for a wide range of frequencies since its vibration frequency is highly dependent on the mounting tissue-type and there exist instances where LRA vibrations are undetected for each tissue-type.

In contrast the displacement amplitude and acceleration amplitude are the best and second-best measures, respectively, for predicting participants' ability to detect feedback. Both measures perform similarly, correctly predicting if vibrations were detected $77.8 \%$ and $75.5 \%$ of the time when using the displacement and acceleration amplitudes, respectively. Slight differences in performance although interesting are not necessarily significant considering testing was conducted with only 3 participants. Further testing would be needed to more definitively establish which measure acts as a better predictor.

The displacement and acceleration amplitude are likely better predictors than the vibration frequency because they increase linearly with the voltage across the LRA. Therefore these measures account for the intensity of feedback which would affect whether or not feedback is detected by participants. It may be that the displacement is slightly more meaningful because it directly related to the amount of deflection applied onto the skin and therefore to the mechanoreceptors which detect the vibrations.

Results for ERM Data: Since only the ERMs will be used in future studies, it would be useful to evaluate the effect of different measures on specificity and sensitivity. However, since vibrations provided by the ERM were detected $100 \%$ of the time, it is impossible to effectively do so. The lack of no detection events means that the same arbitrarily low cutoff value (i.e. 0) could be used for all the predictor measures considered and yield specificities and sensitivities of 1 . However this would in no way be an adequate indicator of predictor's effectiveness. Instead this analysis should be conducted in the next study which will have more participants and would hopefully 
have some instances where feedback is not detected.

\subsection{Summary}

This chapter conducted tests with two types of tactors (ERM and LRA) on the three types of tissue over which these tactors would be mounted in future studies. In doing so, a deeper understanding of the differences in vibration characteristics observed across different tissue types was developed, two predictors of participants' ability to detect feedback were identified and the tactor type to use in future studies was selected.

The ERM and LRA behave according to their respective datasheets for the majority of parameters, but the recorded acceleration amplitudes are greater for the ERM than the LRA in this experimental setup when they are in fact supposed to be smaller according to their datasheets. This is due to differences between Precision Microdrives' set-up and the set-up used in this study. As was expected, the LRA vibration frequency does not vary with the voltage supplied and is much greater than the ERM vibration frequency, which, in accordance with its datasheet, increases as the supply voltage increases. These differences in vibration frequency may be one of the reasons why the ERM vibrations are better felt than the LRA vibrations which theoretically would only sometimes be sensed by mechanoreceptors in the skin.

Based on the observed results, the ERM has been selected as the tactor to be used in future studies; this is due to its greater acceleration and displacement amplitudes, increased percentage of vibrations detected when provided by the ERM, and reduced effective inter-participant and inter-body site variations. Feedback with voltages across the ERM of $0.53 \mathrm{~V}$ or greater should be used in future studies to ensure that feedback is always felt by participants.

With regards to predictor values for the participants' ability to sense biofeedback, 
the displacement and acceleration amplitude were considered the two best predictors because they had the greatest percentage correct predictions (sum of sensitivity and specificity). However, more tests need to be conducted to definitively determine whether the displacement or acceleration amplitude is most effective in predicting feedback detection. As it stands, this study only comprised of 3 participants, thus a study with a greater participant population would allow for proper comparison between the considered predictors, as will be done in future studies. 


\section{Chapter 4}

\section{Body Location Optimization for Vibrotactile Feedback}

\subsection{Objectives}

Although some research exists to determine the best type of information coding method to be use in balance feedback, no study has compared in depth various feedback locations on the body to determine the feedback location that lends itself to improved balance measures (e.g. reduced tilt and reaction time, or increased time spent in the deadzone). Instead, the choice of location for feedback in most studies was often not supported or justified [6] [7]. One study did compare the effects of placing the feedback system on the shoulder to that of the waist; however, the feedback intensity was coded in two different ways for both locations (a greater feedback intensity on the shoulder was communicated through a higher vibration duty cycle, whereas greater feedback intensity was communicated by activating a tactor placed higher on the waist) [10]. By varying both the type of information coding and the feedback location, the effects of the information coding methods and body location become confounded, thereby preventing the adequate ability to discern which location lends itself to better balance feedback.

This chapter investigates the general ability of humans to sense and interpret 
vibrotactile feedback using a four-tactor system placed on areas commonly used for balance enhancement communication; namely the wrist, neck and shoulders area, as well as the most predominant feedback location, the waist [6] [10] [43] [44]. The ankle was also included as a location of interest in this study not only because its performance on balance improvement has yet to be compared to other locations' performance, but also since it is easy to implement, offers low-level obtrusiveness and a relatively constant orientation when walking allowing for easier mapping of feedback to balance. Comparisons of performance on parameters related to balance improvement applications over such a wide range of feedback locations have not been done before.

The best location for vibrotactile feedback will be determined by comparing the following criteria: reaction time, ability to detect feedback, and ability to discern which tactor was activated at which intensity. The effect of Body-Mass Index (BMI) on the ability to sense feedback will also be evaluated by assessing the correlation between the subjects' BMI and their respective performance on each of the evaluation criteria aforementioned. Finally, the ability to predict if feedback will be felt by the subject based on the recorded vibration displacement and acceleration amplitudes will also be investigated.

Using the outcomes of the research presented in this chapter, two optimum feedback sites will be selected for further investigation. Two wearable tactor arrays will be built to offer feedback on these body sites to subjects for balance enhancement studies. The ensuing balance enhancement experiments will be presented in Chapter 5. 


\subsection{Methods}

\subsubsection{Subjects}

Ten healthy subjects ( 5 males and 5 females) with no reported neurologic conditions, with ages ranging from 21 to 28 years (average 24.3 years), volunteered to participate in the experiment. All subjects but one were right handed. Participant demographics are outlined in Table 4.1. All subjects read and signed a consent form, stating that they were well informed of the types of activities this study entailed and of the data's use in furthering this research, in accordance with ethics clearance provided by Carleton University's Research Ethics Board (protocol clearance number 101290).

Table 4.1: Participant Demographics

\begin{tabular}{lc}
\hline Variable & Value \\
\hline Gender & \\
Male & 5 \\
Female & 5 \\
Age Range & $21-28$ \\
Age Mean (STD) & $24.3(1.9)$ \\
BMI Range & $20.4-29.6$ \\
BMI Mean (STD) & $24.6(3.2)$ \\
\hline
\end{tabular}

\subsubsection{Procedure}

This study aims to compare the effectiveness on feedback sensing of every feedback location presented in existing vibrotactile feedback studies. The only location previously used in vibrotactile feedback applications that is not included in this study is the head since, following preliminary testing, it was considered too disruptive and uncomfortable. It is important to note that the existing device which makes use of 
vibrotactile feedback on the head only provides vibrotactile feedback for a specific tilt range; for other tilt ranges, feedback was communicated through visual or auditory means thereby not complying with the tests at hand and greatly reducing the discomfort [15].

Therefore, as part of this study, 4 body locations (neck, wrist, waist and ankle) were tested to compare the performance of existing feedback locations used in balance improvement systems with the addition of the ankle as a novel site of investigation. Feedback on the ankle and wrist was unilateral, and therefore only provided to one ankle and one wrist.

Four tactile vibration actuators (tactors) were used to provide feedback on each body location, placed as shown in Figure 4.1. Each tactor was an eccentric rotating mass actuator (ERM) (Precision Microdrives, 310-003), and therefore exhibited greater movement along the skin surface. ERMs were used in this and future studies since it was found in the previous chapter that ERMs are more easily sensed than linear resonant actuators (LRA).

Each tactor was held in place using a bandage (MediCare Heavy Fabric Adhesive Bandage) with a non-adhesive pad to decrease the effect of vibration damping provided by the attachment method, thereby allowing for greater tactor motion parallel and perpendicular to the surface of the skin. To ensure similar sub-bandage pressures, and thereby ensuring comparable resistance to tactor motion for each location, a tension of 2 Newtons, measured using a dynamometer, was applied to each bandage (it has been shown that even when using different bandage application methods, the sub-bandage pressures have very small relative differences for a given bandage tension [35]). The tactor attachment used in this study is the same as the one outlined in greater depth in the previous chapter.

Once the tactors were attached to the body location to be tested, subjects were given a practice round to get accustomed with identifying the tactor activated and its 


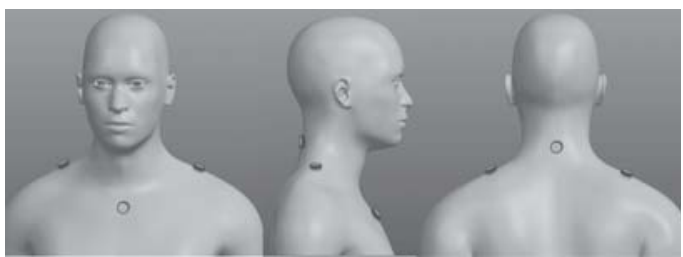

(a)

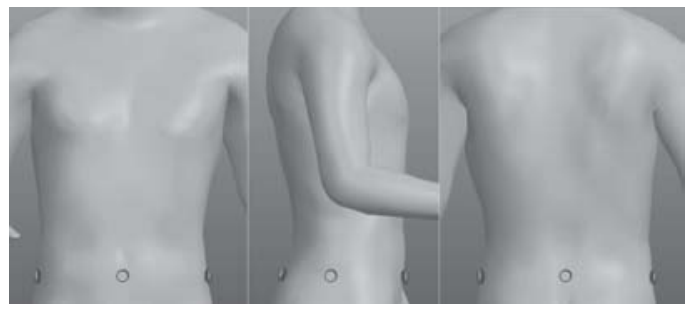

(c)

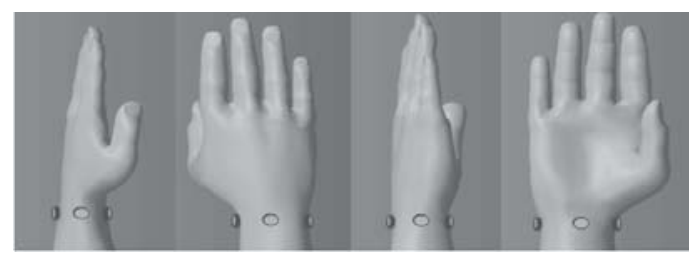

(b)

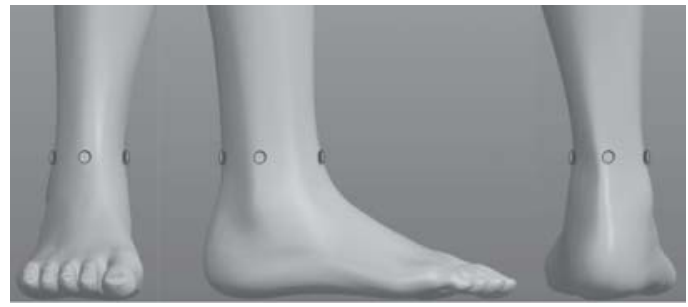

(d)

Figure 4.1: The tactor placement for each testing location was as shown above for the (a) neck (b) non-dominant wrist (c) waist and (d) left ankle.

vibration intensity. This practice round activated each of the 4 tactors at their low and high intensities in a preset order known to the subject. This preset activation order was repeated 3 times, thereby allowing subjects to feel the low and high vibration intensities on each tactor 3 times each before beginning the test.

As part of the test, subjects were invited to indicate which tactor was activated and at which intensity it vibrated, by pressing the corresponding button in the user interface (shown in Part D of Figure 4.2). The user interface has 8 buttons (a set of two buttons per tactor) arranged in a formation similar to the one in which the tactors are attached to participants' bodies. The blue button should be pressed when a low vibration intensity is felt and the red button should be pressed when high feedback intensities are felt. Subjects were instructed to press the button as quickly as possible when feedback was detected for reaction speed identification. Subjects were also encouraged to return their hand to the same position between tactor activation events to reduce variations in results between body locations.

The test for each body location consisted of 24 tactor activation events; there were 3 activation events for each tactor (1, 2, 3 or 4) at each intensity (low or high). 
The order of activation of each tactor/intensity combination was randomized for each body location, and was different across subjects to prevent testing order effects on the results. Furthermore, to prevent subjects from being able to predict when the next vibration would occur, delays between tactor activation events varied in length, were randomly ordered for each body location, and differed between participants. To further prevent testing bias, the order in which each body location was tested was randomly varied from one participant to another.

As part of this study, each participant underwent 24 tactor vibration activation events per location tested, for a total of 4 locations. Therefore each participant was provided with 96 tactor activation events to identify, and the measures for 960 tactor activation events were used in this study's results analysis.

\subsubsection{Instrumentation}

This study made use of 4 tactors that vibrated with a frequency proportional to the input DC voltage (varying from $50-100 \mathrm{~Hz}$ over the voltage range used in this study) to provide subjects with vibrotactile feedback. In order to provide a range of DC voltages using a digital pin to power the tactor, a PWM signal can be used; this signal would then be low pass filtered thereby providing an output DC voltage of magnitude equal to the PWM signal's RMS value. This PWM signal was provided by the Arduino Mega (shown in Figure 4.2) and had a frequency of $31,372.55 \mathrm{~Hz}$ which allowed for effective low pass filtering of the signal. The PWM signal supplied by the Arduino was low pass filtered using Low Pass Filter 1, shown in Figure 4.3, with cutoff frequency of $106 \mathrm{~Hz}$ thereby converting the PWM output to a DC voltage needed to power the tactor. Although a low-enough cutoff frequency was required, this particular cutoff frequency was incidental; the capacitor value of $0.1 \mu F$ was chosen because it has an impedance of $50.7 \Omega$ similar to the recommended terminator resistance of $49.9 \Omega$ and the resistance value of $15 k \Omega$ was chosen to ensure that the 


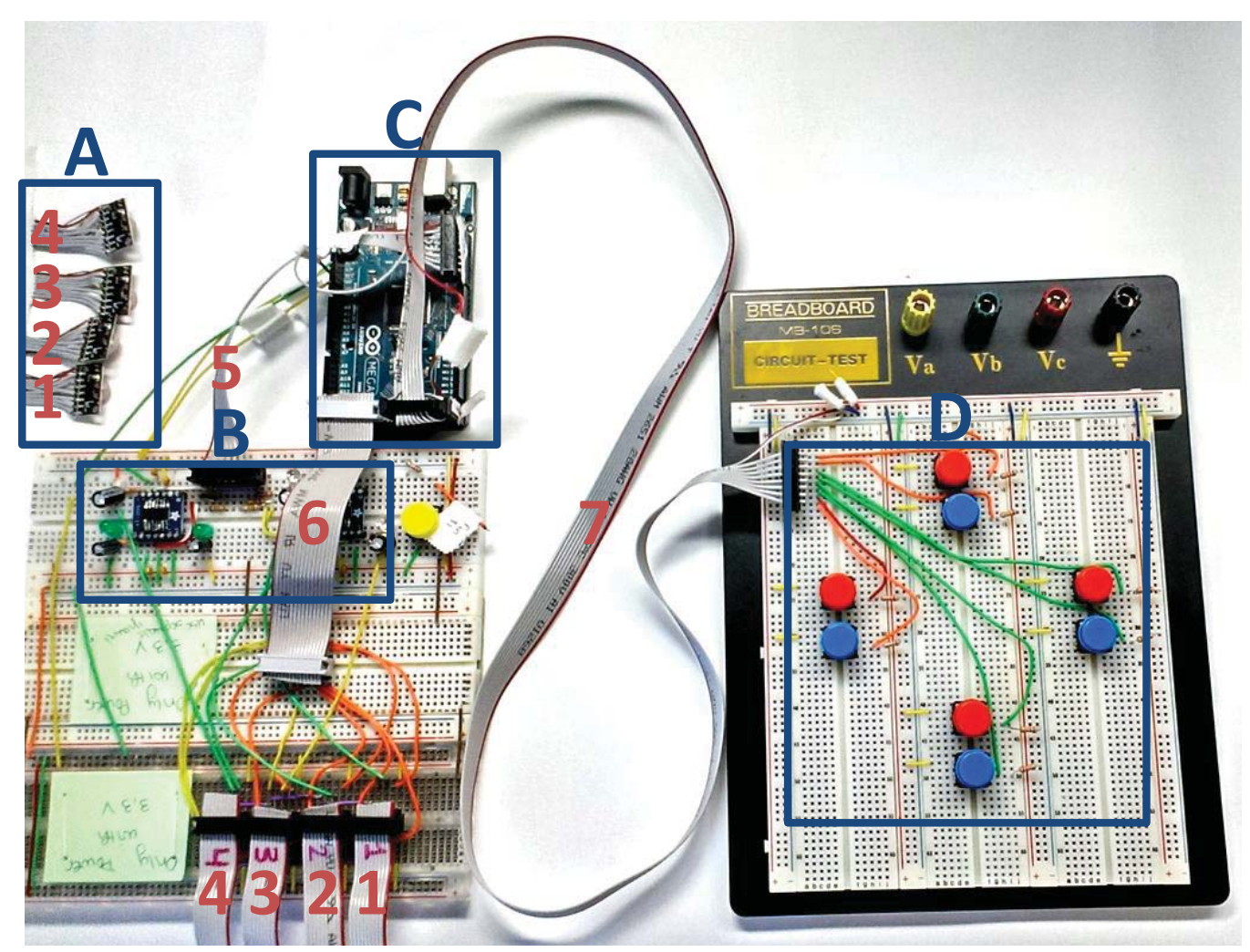

Figure 4.2: This was the circuit used to conduct this study. The accelerometers/tactors (A), tactor powering circuit (B), Arduino (C) and user interface (D) are depicted. Ribbons 1, 2, 3 and 4 are used to link the accelerometers and tactors to the breadboard. Ribbon 5 provides the PWM input for the tactor powering circuits. Ribbon 6 allows communication between the Arduino and the accelerometers. Ribbon 7 allow the Arduino to read button-press instances from the user interface. This circuit's schematic representation can be found in Appendix D.

current draw from the Arduino pin was far below the $40 \mathrm{~mA}$ limit and to ensure that the rise time was in order of a few milliseconds (much smaller than typical human reaction times).

Low Pass Filter 1's output signal was then passed to the MAX4019 buffer which ensured that the current draw on the Arduino digital I/O pins was lower than the allowed limit of $40 \mathrm{~mA}$ while still ensuring that the current requirements for the tactor activation could be met, since the Arduino Mega's Vcc pin's current limit of $200 \mathrm{~mA}$ used to power the MAX4019 is well above that of the tactors' (up to $120 \mathrm{~mA}$ ) [45] [46]. The MAX4019 was chosen as amplifier for the circuit because it 


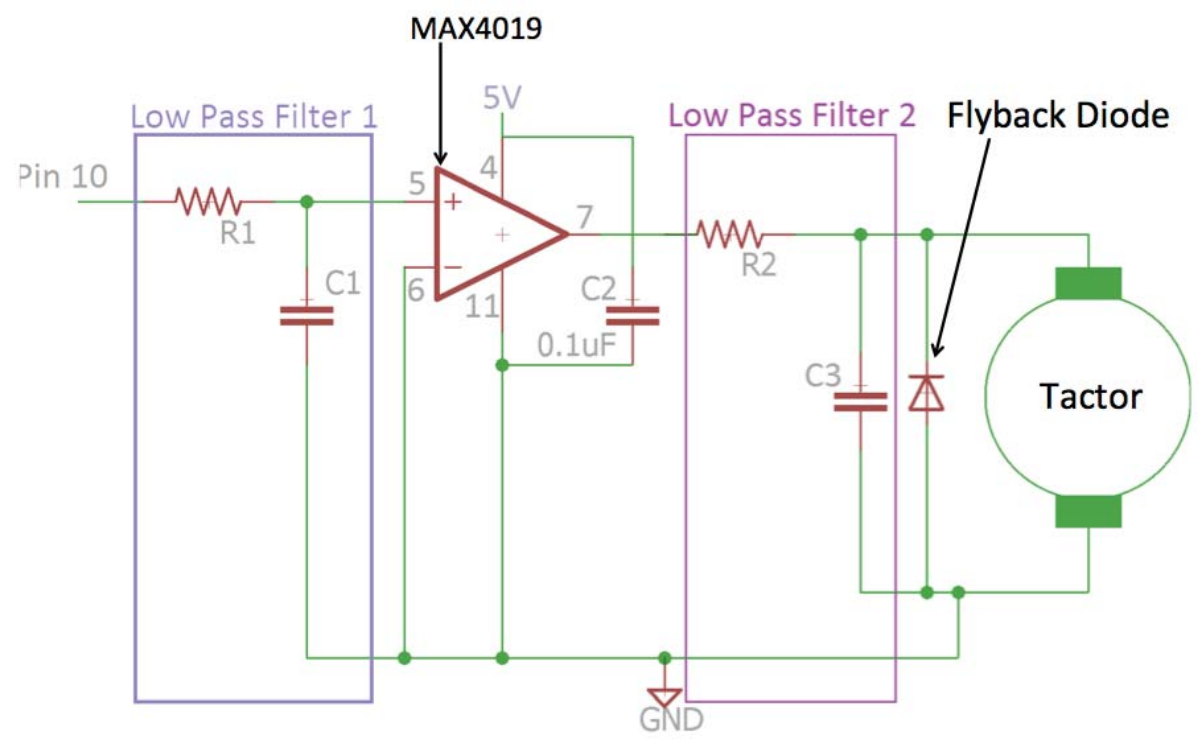

Figure 4.3: This is the circuit used to power one ERM using a PWM signal filtered by the first and second low pass filters and amplified by the MAX4019 buffer. The pin numbers on the operational amplifier correspond to the pin numbers for the first operational amplifier on the SOIC chip for the MAX4019. For the other two operational amplifiers on the chip, the corresponding pin connections are interchangeable as follows: 5/10/12,6/9/13 and 7/8/14, all other pin connections to the ground of power source only need to be made once per MAX4019 chip. The resistor and capacitor values for the circuit are: $\mathrm{R} 1=15 \mathrm{k} \Omega, \mathrm{R} 2=33$ $\Omega, \mathrm{C} 1=0.1 \mu \mathrm{F}, \mathrm{C} 2=0.1 \mu \mathrm{F}, \mathrm{C} 3=100 \mu \mathrm{F}$.

can be powered by the Arduino's 5V Vcc pin whereas other amplifiers that provided currents large enough to power the tactors often required greater powering voltages. Furthermore, the MAX4019's output voltage for a load resistance of $50 \Omega$ (similar to the load resistance (tactor resistance) in this circuit) can range from 0.04 to $3.4 \mathrm{~V}$, therefore the MAX4019 can easily power the tactors since they should not be powered with voltages in excess of $1.8 \mathrm{~V}$ [46]. The Vcc pin for the MAX4019 is bypassed to ground using a $0.1 \mu \mathrm{F}$ capacitor to reduce the amount of circuit ripple.

The MAX4019 buffer's output was then filtered using a second low pass filter (Low Pass Filter 2 in Figure 4.3) with cutoff frequency of $48 \mathrm{~Hz}$ to further attenuate 
the effects of the PWM signal but also to remove the operational amplifier's noise artefact, thereby providing a smoother DC voltage across the tactors. A lower cutoff frequency than for Low Pass Filter 1 was used to further restrict the high frequency behavior of the system. In addition, the chosen $\mathrm{R} 2$ and $\mathrm{C} 3$ values used in the low pass filter ensured a rise-time once again only a few milliseconds in length. To ensure that all tactors were powered similarly, the unused operational amplifiers on the second MAX4019 circuit were connected as if they were powering tactors, as seen in Figure 4.2 .

It is important to note that the input range of the MAX4019 is limited by its output range capabilities; for a $5 \mathrm{~V}$ supply voltage, the output voltage can range from $0.04 \mathrm{mV}$ to $3.4 \mathrm{~V}$, therefore for a gain of 2, the input $\mathrm{DC}$ voltage can only range from 0 to $1.7 \mathrm{~V}$. This means that the PWM signal can have a duty of $0 \%$ to $\frac{1.7 V}{5 V} \cdot 255=86.7$. Therefore in the analogWrite function, the PWM numerator should not exceed 86, because for greater DC voltages than this, the output voltage is a non-linear function of the input.

An accelerometer (ADXL 345) was attached to each tactor (according to the circuit shown in Figure 4.2) to monitor if the tactors were in fact activated for each test and communicated with the Arduino using the SPI communication protocol. For each tactor activation instance when the tactor did not vibrate, the data pertaining to that instance was removed from the overall performance of the subject. Therefore, if the subject did not feel the feedback (because none was effectively given) and did not press a button to signal having felt the feedback, this did not get recorded as a "no feedback felt" instance, but rather was excluded from the dataset analyzed.

The accelerometer data was also used to investigate the feasibility of predicting whether or not a subject should have felt the feedback given by the system through the use of the tactor's acceleration and displacement amplitudes. These have been shown to be effective predictors of a subject's ability to sense feedback in the previous 
chapter. To ensure that the subject's pressing of a button on the user interface was not missed while recording the tactor acceleration, the acceleration profile for the tactor activated was obtained only once the subject had pressed a button thereby indicating that feedback was felt.

To evaluate subject performance in sensing feedback and identifying which tactor was activated at which intensity, the subjects had to press one of the eight buttons on the user interface (shown in Figure 4.2) which sent to the Arduino information regarding whether or not feedback was detected (button pressed or not), the perceived tactor activated and its perceived vibration intensity. To reduce reaction time delays, the buttons were placed in a similar orientation to that of the tactors on the body to enable easier mapping between both interfaces. For each tactor, there were two buttons on the user interface: the blue button corresponded to low vibration intensity and the red button corresponded to high vibration intensity.

During the test, the subject would have to press the button corresponding to the tactor activated and its vibration intensity. This perceived information was then recorded by the Arduino and later compared to the actual data regarding the tactor activated and its vibration intensity. This provided the percent detected feedback, the percent of correct tactor identifications and the percent of correct vibration intensity identifications. Reaction times were measured by the time difference between when the tactor was activated and when a button was pressed. Although this measure was not a perfect representation of the user's response because there was an added delay associated with mechanically displacing the button enough for the movement to be recognized, it was adequate in the case where one user's performance for one body location was compared to that same user's performance for another body location, because the delays would be similar from one location to another. This measure did however prevent the nominal comparison of one subject's reaction time to another subject's. 


\subsubsection{Arduino Programming}

The entirety of this test is conducted using the Arduino code shown in Appendix E. This code allows the researcher to change the order in which tactors are activated along with their activation intensity and the time delay between tactor activations, so that the testing order across body locations and participants can be randomized to reduce testing order effects on the results. In order to run the test, the researcher must upload the code to the Arduino, then he or she must open the serial monitor. This will allow the code to begin. A number will appear at the top of the window's script. This number indicates which body location will be tested first; the codes along with their corresponding meaning are found in Table 4.2. Once the tactors are attached and the participant has been told how the tactors relate to the buttons shown in Part D of Figure 4.2, the researcher presses the yellow button shown as part of the circuit in Figure 4.2 to allow the code to move on to testing the participant's response to vibrotactile feedback on this location.

After a randomly-allocated time delay, one of the tactors will be activated and the Arduino will wait for 4 seconds to see if the participant presses any of the button in Part D. If the participant presses one of the buttons in Part D, then the microcontroller (Arduino) will record the reaction time and proceed to read the acceleration profile of that activated tactor before stopping tactor vibrations. If the participant does not press any of the button in Part D of the circuit, then it is assumed that the vibrotactile feedback was not sensed and this lack of reaction is recorded then the acceleration profile of the tactor is recorded before the tactor is turned off. Once the tactor is turned off, the information pertaining to the test is printed; this includes the information pertaining to actual activation (the index of the tactor activated and the intensity of activation), perceived activation (the index of the tactor the participant thought was activated and the perceived vibration intensity), the reaction time and 
Table 4.2: Meaning of Arduino Program's Location Codes

\begin{tabular}{|c|c|c|}
\hline & $\begin{array}{l}\text { Body } \\
\text { Location }\end{array}$ & Attachment Points \\
\hline 11111111 & Neck & $\begin{array}{l}\text { Front: Inferior to manubrium's jugular notch } \\
\text { Right: Right trapezius muscle's distal end } \\
\text { Back: Superior to vertebra prominent } \\
\text { Left: Left trapezius muscle's distal end }\end{array}$ \\
\hline 33333333 & Wrist & $\begin{array}{l}\text { Front: Posterior distal end of the forearm } \\
\text { Right: Styloid process of the radius } \\
\text { Back: Anterior distal end of the forearm } \\
\text { Left: Styloid process of the ulna }\end{array}$ \\
\hline 44444444 & Waist & $\begin{array}{l}\text { Front: Inferior to the umbilicus } \\
\text { Right: Right iliac crest } \\
\text { Back:L4 vertebra } \\
\text { Left: Left iliac crest }\end{array}$ \\
\hline 55555555 & Ankle & $\begin{array}{l}\text { Front: Extensor digitorium longus meeting point } \\
\text { with talocrural region } \\
\text { Right: Proximal to medial malleolus of the fibula } \\
\text { Back: calcaneal tendon } \\
\text { Left: Proximal to lateral malleolus of the fibula }\end{array}$ \\
\hline
\end{tabular}

the acceleration profile. To represent each tactor's activation profile, 200 data points were recorded at a sampling frequency of $1,776 \mathrm{~Hz}$, which allowed for approximately 10 tactor vibration cycles to be captured per test. Then, after another randomly pre-selected time delay, another tactor is activated, until all the tests for one location are completed and the location code for the next location appears.

In order to allow participants to test how low and high vibration intensities feel and how the buttons relate to the tactors on their bodies, the code is run independently for each tactor location once with a preset activation order and intensity known to the participant. The tactor activation orders and intensities are then altered for 
the actual test, to ensure the participants' inability to predict which tactor will be activated at which intensity.

\subsubsection{Data Analysis}

Data analysis was performed through post-processing using MATLAB software (Release 2014b) and IBM SPSS Statistics for Windows (Version 24.0). Using MATLAB, the data for all the locations was filtered to remove the data recorded for events when the tactor did not actually vibrate. From this data, for each subject and each body location tested, 4 measures were calculated: mean reaction time, percentage detected feedback, percentage correctly identified tactor and percentage correctly identified intensity. The percentage detected feedback is the ratio of the number of events for which a button was pressed by the participant (and therefore feedback was detected) to the number of events where a tactor was activated. The percentage correctly identified tactor is the ratio of the number of events where the participant correctly identified the tactor (front, right, back or left) activated to the number of events where a tactor was activated. Finally, the percentage correctly identified intensity is the ratio of the number of events where the participant correctly identified the activation intensity (low or high) to the number of events where a tactor was activated.

The average and standard deviation of all subjects' data for each evaluation criteria were taken as the overall evaluation criteria values for each body location. To allow for a weighted total score to be allocated to each body location, these evaluation criteria values were converted to a common-scale. Therefore each location was awarded a score from 0 to 1 for each evaluation criteria based on its mean performance across subjects. For each evaluation criteria presented as a percentage (percentage detected feedback, correctly identified tactor and correctly identified intensity), a score of 1 was given to the location with the greatest percentage, and all other body locations' scores were assigned the ratio of their corresponding percentage to the highest percentage 
as shown in Equation 4.1.

$$
\left[\begin{array}{ll}
P_{\min } & P_{\max }
\end{array}\right] \longmapsto\left[\begin{array}{ll}
\frac{P_{\min }}{P_{\max }} & 1
\end{array}\right]
$$

For the reaction time, the location with the lowest reaction time received a score of 1 and the location with the highest reaction time received a score of 0 ; other locations' scores were linearly interpolated based on their respective reaction times as outlined by the mapping relationship shown in Equation 4.2 .

$$
\left[\begin{array}{ll}
t_{r_{\max }} & t_{r_{\min }}
\end{array}\right] \longmapsto\left[\begin{array}{ll}
0 & 1
\end{array}\right]
$$

By giving a score between 0 and 1 for each of the evaluation criteria, it was possible to attribute a weight to each evaluation criteria based on its importance in the final design, and find each body location's total score $(T S)$ as the weighted sum of each evaluation criteria's score for that location, as shown in Equation 4.4.

$$
\begin{gathered}
T S=\sum_{i=1}^{4} W_{i} \cdot S_{i}=W_{D} \cdot S_{D}+W_{T} \cdot S_{T}+W_{I} \cdot S_{I}+W_{R} \cdot S_{R} \\
T S=\sum_{i=1}^{4} W_{i} \cdot S_{i}=1 \cdot S_{D}+3 \cdot S_{T}+0.8 \cdot S_{I}+1 \cdot S_{R},
\end{gathered}
$$

where, as outlined in Table $4.3, W_{D}$ was the percentage detected feedback weight, $S_{D}$ was the percentage detected feedback score for that location, $W_{T}$ was the percentage correctly identified tactor weight, $S_{T}$ was percentage correctly identified tactor score for that location, $W_{I}$ was the percentage correctly identified intensity weight, $S_{I}$ was the percentage correctly identified intensity score for that location, $W_{R}$ was the reaction time weight, and $S_{R}$ was the reaction time score for that location.

Another total score $\left(T S_{P}\right)$ was calculated as shown in Equation 4.6 to take into account the implementation practicality for each of these proposed systems, another 
Table 4.3: Evaluation criteria's scores and weights used in total score (TS) calculation

\begin{tabular}{lcccc}
\hline Evaluation Criteria & $\begin{array}{c}\text { Score } \\
\text { Variable }\end{array}$ & $\begin{array}{c}\text { Score } \\
\text { Range }\end{array}$ & $\begin{array}{c}\text { Weight } \\
\text { Variable }\end{array}$ & $\begin{array}{c}\text { Weight } \\
\text { Value }\end{array}$ \\
\hline \% detected feedback & $S_{D}$ & $0-1$ & $W_{D}$ & 1 \\
\% correctly identified tactor & $S_{T}$ & $0-1$ & $W_{T}$ & 3 \\
$\%$ correctly identified intensity & $S_{I}$ & $0-1$ & $W_{I}$ & 0.8 \\
Reaction time & $S_{R}$ & $0-1$ & $W_{R}$ & 1 \\
Practicality/ ease of use & $S_{P}$ & $0-1$ & $W_{P}$ & 2 \\
\hline
\end{tabular}

important criteria in the development of a feedback system.

$$
\begin{gathered}
T S_{P}=\sum_{i=1}^{5} W_{i} \cdot S_{i}=W_{D} \cdot S_{D}+W_{T} \cdot S_{T}+W_{I} \cdot S_{I}+W_{R} \cdot S_{R}+W_{P} \cdot S_{P} \\
T S_{P}=\sum_{i=1}^{5} W_{i} \cdot S_{i}=1 \cdot S_{D}+3 \cdot S_{T}+0.8 \cdot S_{I}+1 \cdot S_{R}+2 \cdot S_{P}
\end{gathered}
$$

where $W_{P}$ was the implementation practicality weight and $S_{P}$ was the implementation practicality score for that location.

In allocating the weights to each evaluation criteria (summarized in Table 4.3), considerations for the system's final application, balance improvement, played a key role. The criteria corresponding to correctly identifying the tactor activated was deemed the most important by far and given a weight $\left(W_{T}\right)$ of 3 , because it is the only one that, if perceived poorly in the case of a balance application, could exacerbate the balance issues of the wearer. For instance, if the person was falling towards their left, and they perceive feedback as telling them they were falling towards the right, they would compensate by leaning further left, therefore worsening their already unbalanced state. The correct intensity criteria had the lowest weight $\left(W_{I}\right)$ of 0.8 , since so long as the user recognizes the presence of feedback, they will continue to 
adjust their posture, if at a slower rate, until the vibrations end. This criteria does not cause balance issue exacerbation: at worst it increases the amount of time needed to reach a balanced state. Both the reaction time and feedback detection criteria are equally important in improving balance, but still remain much less critical than correctly identifying the activated tactor since their failure to perform poses no added detriment to the user's balance state and therefore received weights $\left(W_{D}\right.$ and $\left.W_{R}\right)$ of 1 . However, it is important for the user to be able to react to feedback quickly, because it is easier for users to reorient their body when they are closer to their target balanced posture and it is important for the users to be able to detect the feedback provided to them, otherwise the device was of no added use. The weight associated with the implementation practicality $\left(W_{P}\right)$ of 2 was greater than for the non-critical balance criteria since it affects not only if the device can be made to suit a large range of individuals and if the device will function as expected, but it also takes into account user likelihood to wear the device. The simple and more easily concealed the device is, the more people are likely to wear and use it daily.

Table 4.4: Practicality scores for each location

\begin{tabular}{lc}
\hline Location & Practicality Score, $S_{P}$ \\
\hline Neck & 0.2 \\
Waist & 1 \\
Wrist & 0.83 \\
Ankle & 0.66 \\
\hline
\end{tabular}

Unlike other scores included in the total score calculation, the implementation practicality scores for each location, summarized in Table 4.4, were determined based on the research team's judgment. The neck was allocated the lowest score of 0.2 since it is the location least simple to implement because it does not consist of simply wrapping a device around a body part like the wrist, waist and ankle. It requires that the device be heavy enough to hold the tactors in place during daily use without being 
too heavy to cause discomfort, or requires a harness of sorts around the wearer's chest. Despite the difficulty to implement this device, it was tested in order to compare all body locations previously investigated in balance enhancement research. The waist is the most easily implemented system, in part due to the extensive amount of work done on biofeedback provided on the waist, and in part because it is very similar to a belt and could therefore be easily integrated with regular clothing; it received a score of 1 . The wrists and ankle are less easy to implement than the waist, because the need for a power source large enough to power the tactors throughout the daily use requires either a power source mounted at the feedback location or wires linking the tactors to the power source placed in a more stable body location (e.g. the waist), which is complicated by the large range of motion of both the wrist and ankle during daily activities. The wrist would be more accepted by the population as a feedback location since it is customary to see devices placed on the wrist (like the fitbit or other health monitoring wrist-based devices) and therefore received a score of 0.83 . The ankle received a slightly lower score of 0.66 since it is likely to be less accepted as a feedback location than the wrist or waist due to the fact that very few devices on the market are currently ankle based. Furthermore, it makes wearing shoes that come higher on the ankle uncomfortable or impossible which can become an issue, especially in the winter.

Once the total scores for each body location were tabulated, a repeated measures ANOVA with a Greenhouse-Geisser correction was performed for the set of body location scores for all evaluation criteria to determine if there was a significant difference between the four body locations' performances. Through the use of post hoc paired-samples t-tests using the Sidak correction, it was possible to identify which pairwise comparisons exhibited significant differences.

Furthermore, the change in total score for each body location and its effect on the overall best location was evaluated for varying weights associated to each evaluation 
criteria. This was done to ascertain that, given the relative ordering of the weights (e.g. the weight associated with the correct tactor identification was greater than the weight associated with the reaction time), should the value of these weights change without altering the relationships between them, the best body location for feedback would remain the same. This would therefore negate the potential bias associated with choosing nominal values for the weights.

\subsection{Results and Discussion}

\subsubsection{Identifying Best Body Location for Feedback}

\subsubsection{Total Scores}

The mean values for each evaluation criteria at each body location were found by averaging the results for all participants, and then scores were allocated to each body location based on the conversions outlined in Equations 4.1 and 4.2. From these scores on each evaluation criteria, the total score $(T S)$ which does not take into account the implementation practicality associated with creating feedback devices for each location and the total score which considers implementation practicality $\left(T S_{P}\right)$ we calculated using Equations 4.4 and 4.6, respectively. These total scores help identify which body locations allow participants to better sense feedback and should therefore be further investigated in future studies.

Table 4.5 reports the total scores for each body location for easier identification of differences in location overall performance. It can be seen that the overall best and second best locations for feedback were the waist and ankle, respectively; this was true both when only considering feedback sensing performance and also when considering implementation feasibility. This supports the common decision in the balance feedback field to use the waist as a feedback location. The waist was likely 
the best location because it was the most intuitive and because of the large distance between tactors which makes discerning between activated tactors easier than on the wrist and ankle. The neck and wrist alternate between the third and fourth best options, thereby making them poor alternatives to the waist and ankle.

Table 4.5: Total score excluding practicality $(T S)$ and total score including practicality $T S_{P}$ for each body location

\begin{tabular}{c|cc|c|cc}
\hline Body Location & $T S$ & Ranking & Body Location & $T S_{P}$ & Ranking \\
\hline Waist & 5.64 & 1 & Waist & 7.64 & 1 \\
Ankle & 5.55 & 2 & Ankle & 6.88 & 2 \\
Neck & 5.44 & 3 & Wrist & 6.20 & 3 \\
Wrist & 4.53 & 4 & Neck & 5.84 & 4 \\
\hline
\end{tabular}

Interestingly enough, Table 4.6 shows that though the waist had the greatest total score, there were other locations that performed better when considering individual criteria. For instance, the reaction time was lowest for the waist, but the neck had the greatest percent detected feedback and allowed for greater tactor identification accuracy, and the ankle offered the best intensity identification accuracy. The waist had the lowest reaction time due to the fact that it was easier to map the feedback information to the user interface on this orientation (often the time delay in reaction time was not due to subjects not feeling the feedback, but rather to their delay in deciding which tactor was activated or which vibration intensity was felt). The neck offered similar spacing between tactors as the waist but had less lipid tissue to attenuate the vibration feedback, thereby explaining it having the best performance detecting feedback and discerning the activated tactor. The ankle possibly had the greatest intensity detection because the tactors were placed on a very thin skin layer over a bone, which could involve vibration pickup by bone sensory receptors provided vibration propagation depth through the skin is large enough. Vibration pickup by 
bone sensory systems could amplify the feedback and cause greater felt differences in feedback intensity. More research is needed to identify whether bone sensation is a factor when discussing sensation of small vibrations over skin-covered bone, since no study has studied these effects.

Lastly, it can be seen in Table 4.6 that subjects had a greater difficulty identifying the vibration intensity than the tactor activated, as seen by the lower percentages for correct intensity identification across all body locations. This could be because it is difficult to recall with accuracy the difference between low and high intensity feedback; participants found it easier to identify differences in intensity when two sequential vibration instances of different intensities were provided and they could compare the second vibration instance's intensity to the first. In future studies, to facilitate the detection of low and high vibration intensities, it might be beneficial to have a low vibration intensity triggered as a first warning of dangerous balance state and have the vibration intensity increase if the balance state worsens; having the two feedback intensities given sequentially could make it easier to discern the vibration intensity.

\subsubsection{Location Performance on Evaluation Criteria}

Table 4.6: Mean(SD) reaction time and percentage detected feedback, correctly identified tactor and correctly identified intensity

\begin{tabular}{lccrc}
\hline Evaluation & \multicolumn{3}{c}{ Body Location } \\
Criteria & Neck & Wrist & Waist & Ankle \\
\hline Reaction Time (s) & $1.4(0.4)$ & $1.8(0.4)$ & $1.3(0.3)$ & $1.4(0.3)$ \\
\% Detected & $100(0)$ & $97.5(5.3)$ & $97.9(3.0)$ & $99.2(2.6)$ \\
\% Tactor & $98.3(2.2)$ & $92.8(6.0)$ & $96.3(4.1)$ & $95.8(3.9)$ \\
\% Intensity & $84.9(9.0)$ & $84.7(5.5)$ & $85.0(9.9)$ & $93.3(4.5)$ \\
\hline
\end{tabular}

In order to compare and identify statistical differences between the sample means for each body location, a repeated measures ANOVA with a Greenhouse-Geisser 
Table 4.7: Significance of differences between measures for each body location (Neck,

Wrist, Waist and Ankle) when Greenhouse-Geisser correction is applied

\begin{tabular}{lll}
\hline Measure & $\mathbf{F}$ & $\mathbf{P}$ value \\
\hline Reaction Time & 11.029 & $\mathbf{. 0 0 0}$ \\
\%Detected & 1.725 & .215 \\
\%Tactor & 3.804 & $\mathbf{. 0 2 9}$ \\
\%Intensity & 2.587 & .098 \\
\hline
\end{tabular}

Table 4.8: p values comparing body location means for each evaluation criterion

\begin{tabular}{lllllll}
\hline \multirow{2}{*}{ Evaluation Criteria } & \multicolumn{7}{c}{ P values } \\
& $p_{N-W r}$ & $p_{N-W a}$ & $p_{N-A}$ & $p_{W r-W a}$ & $p_{W r-A}$ & $p_{W a-A}$ \\
\hline Reaction Time & .060 & .296 & .994 & .002 & .038 & .807 \\
\% Detected & .622 & .275 & .920 & 1.000 & .477 & .859 \\
\% Tactor & .095 & .814 & .260 & .481 & .478 & 1.000 \\
\% Intensity & 1.000 & 1.000 & .226 & 1.000 & .009 & .183 \\
\hline
\end{tabular}

correction was performed which produced the p values shown in Table 4.7, where values smaller than 0.05 identify the existence of a significant difference between two or more body locations. Table 4.8 shows the $\mathrm{p}$ values for pairwise comparisons obtained through post hoc testing using the Sidak correction for multiple comparisons, where a $\mathrm{p}$ value smaller than 0.05 identifies a significant difference. The two subscripts for each $\mathrm{p}$ value indicate which two body locations were compared, where $N$ refers to the neck, $W r$ refers to the wrist, $W a$ refers to the waist and $A$ refers to the ankle.

All locations performed statistically similarly when it came to detecting feedback and identifying the vibration intensity as outlined in Table 4.7. Only two measures, reaction time $(\mathrm{p}=.000)$ and the ability to detect which tactor was activated $(\mathrm{p}=.029)$, merit further analysis through post hoc testing to determine which body locations perform statistically significantly differently. From Table 4.8 , it can be found that the reaction times for both the waist and ankle were significantly lower than for the wrist ( $\mathrm{p}=.002$ and .038 , respectively). This was possibly due to the fact that the 
mapping of feedback information to the user interface was less intuitive for the arm since the tactors were not oriented in the same plane as the user interface, and because wrist rotation caused confusion in subjects. Interestingly, no significant differences in performance between the neck, waist and ankle when considering their reaction time performance were observed.

Although the repeated measures ANOVA initially found a significant difference between location performance on the percentage correctly identified tactor measure, through the post hoc test's correction for multiple comparisons, this difference was no longer found to be significant ( $\mathrm{p}$ values of .095 and above). Lastly, although there is a p value smaller than 0.05 for comparison between the wrist and ankle performance on percentage correctly identified intensity seen in Table 4.8, because there was no significant difference $(\mathrm{p}=.098)$ in performance identified in the repeated measures ANOVA whose results are shown in Table 4.7, this result is in fact not significant. This is likely due to a lack of statistical power with regards to the percentage correctly identified intensity. Perhaps conducting testing on a greater sample size would have been needed, but these results are not part of commonly tested metrics in existing studies, therefore an effect size estimate could not be made and the minimal sample size to ensure clinical significance in this study could not be calculated prior to the study.

\subsubsection{Effect of Weights on Total Score}

In this study, the weights were selected heuristically, therefore it is important to determine whether slight changes in the weights can change the feedback scores and rankings significantly. To determine the effect of variations in weights allocated to each evaluation criterion, the weights were varied by \pm 75 percent. Figure 4.4 shows the total score for each body location and how it changed when we varied the criteria weights. It can be seen that within the range of weights for all criteria, the locations' 
order based on their total score remained unchanged (the waist always ranked as the top location, followed by the ankle then the neck). This shows that moderate changes in the weights does not affect the optimal feedback location. However, larger changes in the weights can lead to the changes in locations' order based on their total score; for instance, for a reaction time weight of about 0.4 , the ankle exhibits a greater total score than the waist, whereas for greater reaction time weights, the ankle has a lower total score than the waist. Furthermore, for correct intensity weights it can be seen that for some weight smaller than 0.2 the neck would become the second ranking location and the ankle the third, whereas for a weight greater than 1.4, the ankle would become the top ranking location and the waist the second.

\subsubsection{BMI Effect on Ability to Sense Feedback}

As a secondary outcome, the impact of participants' BMI on their ability to sense feedback was investigated. This was done to identify whether differences in future patient BMI and therefore body composition could have an impact on their ability to use the feedback system. To do this, a linear regression analysis was conducted and correlation coefficients were found between participants' BMI and their measured values for each evaluation criterion. Correlation coefficients of magnitudes nearing 1 demonstrate a strong linear relationship between the two measures compared whereas correlation coefficients of magnitudes near 0 show very weak or non-existent linear relationships between the measures compared.

To illustrate the relationships between BMI and each evaluation criteria, Figure 4.5 shows the plots of each individual participant's measured value for each evaluation criteria as a function of BMI. It would seem from this figure that BMI had no effect on the subjects' ability to sense feedback regardless of the body location considered or the evaluation criteria of interest. In fact, there was a poor correlation between BMI and any of the evaluation criteria of interest, with correlation coefficients smaller 

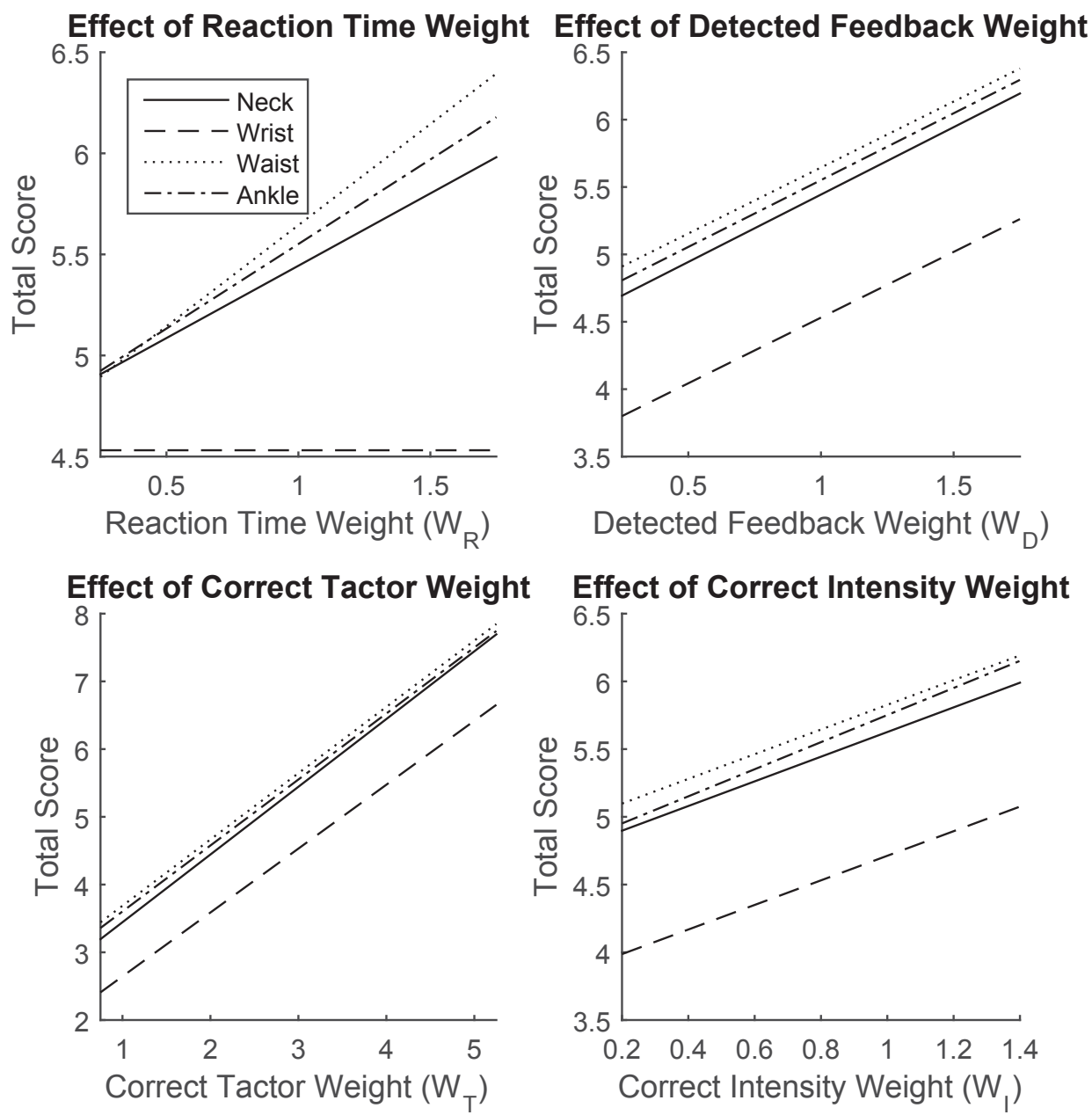

Figure 4.4: The total scores for each body location are given as a function of the criteria weight, each inset identifying the effect of one criterion's weight on each location's total score given that all other criteria weights are fixed.

than 0.485 and as small as 0.015 , as shown in Appendix F. Furthermore, there is no significant linear relationship between any evaluation criterion and BMI, regardless of the body location considered, since no p value obtained through the linear regression analysis is smaller than 0.05, once again shown in Appendix F.

Therefore participant BMI may not be an adequate indicator of participants' sensitivity to biofeedback. However, tests with participants exhibiting a greater range in BMI would need to be conducted to ensure that BMI bears in no way an effect on participants' ability to detect feedback. 

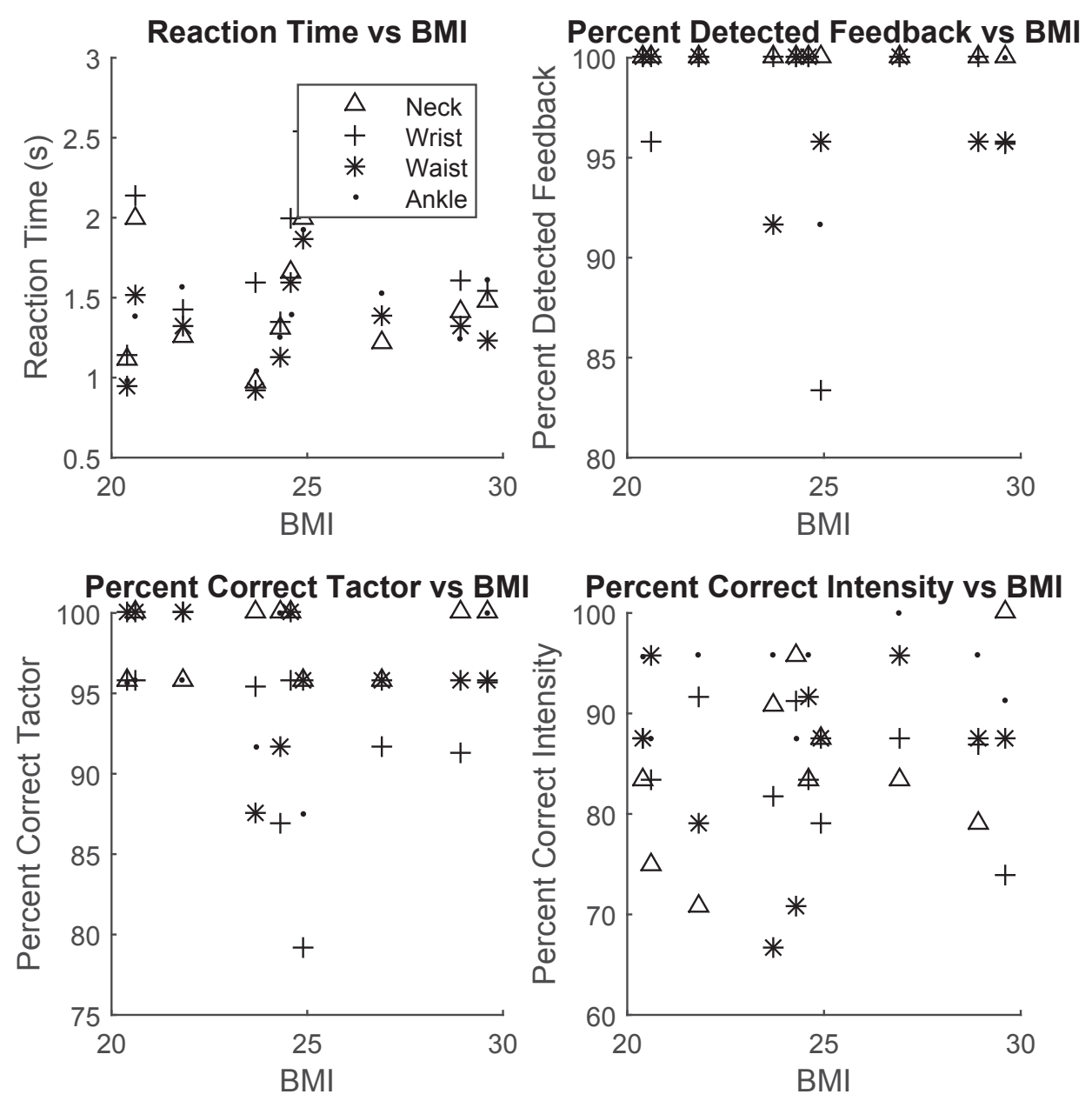

Figure 4.5: The above plots depict the relationships between the performance on each evaluation criterion of each subject and the respective subject's BMI.

\subsubsection{Ability to predict if feedback will be detected}

Since the previous chapter found the acceleration and displacement amplitudes to be effective predictors of a participant's ability to detect feedback in a group of 3 participants, this study investigated these predictors' effectiveness in a larger group. In addition, the acceleration and displacement amplitudes adjusted for participants' BMI were initially considered as predictors of interest. In order to adjust the acceleration and displacement amplitudes with respect to BMI, a strong linear relationship between each predictor and BMI needed to exist. Through a linear regression analysis 
conducted using SPSS, it was found that no consistent significant linear relationship existed between predictors and BMI (see Appendix G for details). Therefore, acceleration and displacement amplitudes adjusted for participants' BMI could not be considered as predictors.

To evaluate the effectiveness of acceleration and displacement amplitudes as predictors of participants' ability to detect feedback, a sensitivity and specificity analysis was conducted in the method outlined in the previous chapter. The variation of sensitivity and specificity as a function of the cutoff acceleration amplitude value is shown in Figure 4.6a. In addition, the variation of sensitivity and specificity as a function of the cutoff displacement amplitude value is shown in Figure 4.6b.

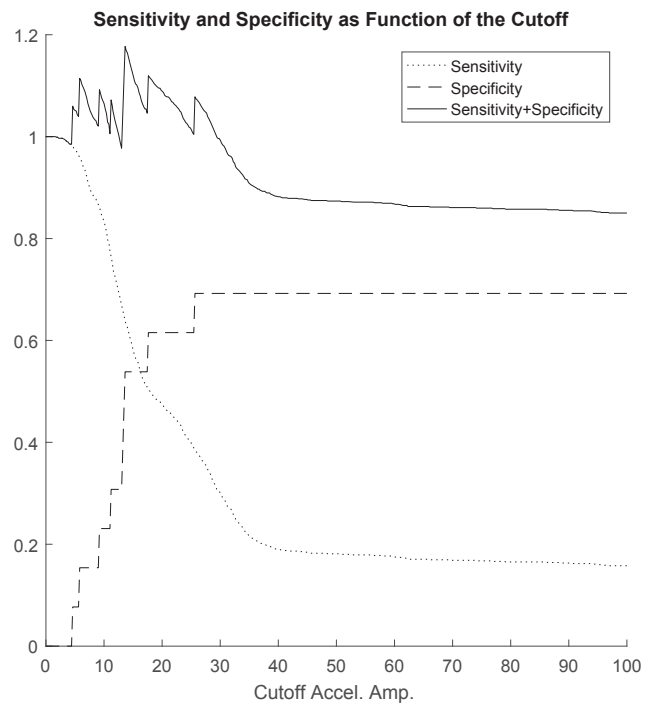

(a)

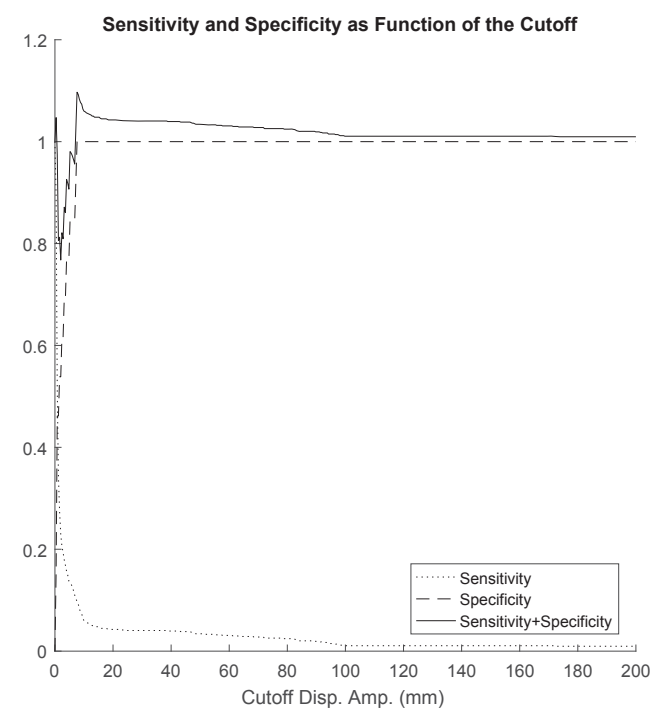

(b)

Figure 4.6: The variations in sensitivity, specificity and their sum is shown as a function of the cutoff value used to identify a vibration event as being detected or not by a participant for both the (a) acceleration and (b) displacement amplitudes used as predictors.

It was found that the vibration acceleration and displacement amplitudes, contrary to the previous chapter's results, did not accurately predict if the feedback would be detected or not. For both predictors, depending on the cutoff value used to decide if feedback will be felt or not, either high sensitivity or high specificity could be 
obtained, but never a reasonable combination of both. This meant the cutoff value for detecting feedback could be low, causing all feedback to be predicted as felt and obtaining a high sensitivity and null specificity, or the cutoff could be high predicting all feedback as unfelt thus obtaining a high specificity and null sensitivity.

As previously mentioned, the sum of the sensitivity and specificity is an effective method in classifying the usefulness of a predictor; the closer the sum of the sensitivity and specificity is to 2 , the better the criteria is at predicting the ability to detect feedback. However, as seen in Figure 4.6, the sum in this case was only just above 1 regardless of the cutoff value or the predictor measure, making this a poor predictor value. In fact using acceleration amplitude, participants' ability to detect feedback was detected correctly at most $58.8 \%$ of the time, a much lower prediction accuracy than observed in the previous study $(75.5 \%)$. Similarly, displacement amplitude exhibited a prediction accuracy of at most $54.9 \%$, much lower than the previously reported value of $77.8 \%$. In addition, the range of cutoff values for which these upper range prediction accuracies are observed are very small, which could mean that these cutoff values dependent on the sample population and with the addition of a new participant could easily change. If this were the case, then these measures would be of little use in predicting the ability to detect feedback in people who were not part of this study's sample population.

Perhaps the poor performance in predicting participants' ability to sense feedback was due to the very low number of instances across all participants where feedback was given but not felt, thereby making it difficult to identify the cutoff. It could also have more to do with environmental distractions, like keeping a conversation. It may be worthwhile to investigate which body locations perform best while the subjects are performing secondary tasks, which has been done in other studies [24]. 


\subsection{Summary}

Several tests were performed on participants to identify the ideal feedback location based on their performance on 4 evaluation criteria: mean reaction time, percentage detected feedback, percentage correctly identified tactor and percentage correctly identified intensity. After converting the mean performance of a given location on each individual evaluation criterion to a common scale, the weighted average of these evaluation criteria was found as the total score. In doing so, body locations could be compared for overall performance in order to identify the best locations for feedback, to be further investigated. Additionally, the effects of BMI on participants' performance on each evaluation criterion was determined. Lastly, the usefulness of acceleration and displacement amplitudes in predicting participants' ability to sense feedback, measures found useful in the previous chapter of this thesis, were further researched to see if in a larger sample group this would still be the case.

As expected based on its prevalence in balance feedback systems, the waist was identified as the best overall body location for feedback dues to its overall total score regardless of if implementation practicality was taken into account. Unexpectedly, the ankle, a location to date unused in the balance feedback field, was seen as the second best performing body location due to its overall second highest total score and the significantly greater ability of people in detecting feedback intensity on the ankle as opposed to any other location tested. As for predicting a person's overall performance in each of the evaluation criteria using their BMI, there was no correlation between any of the evaluation criteria and the BMI. Similarly, it was not possible to predict accurately a subject's ability to detect feedback using the vibration acceleration or displacement amplitudes, contrary to what was previously found.

Further tests need to be conducted to determine which of the waist and ankle allows for better mapping to balance improvement applications. The neck location 
will not be included in future balance improvement tests, because it did not perform statistically better than the alternatives, but is much more difficult to implement. 


\section{Chapter 5}

\section{Vibrotactile Feedback Location Effects on Balance}

\subsection{Objectives}

This chapter presents the experiments on the effect of location of vibrotactile feedback on measures of balance using the two body locations shown to perform best in Chapter 4, namely the ankle and waist. The following experiments aim to determine which body location lends itself to easier interpretation and more natural reactions to correct posture, demonstrated through better performance on balance measures. This set of experiments not only compare the performance of two biofeedback locations, but it also aim to ascertain that vibrotactile feedback does in fact improve balancerelated measures. In this study, balance is defined as it is in multiple other balance improvement studies [47] [48] [49] [10] [21] as the ability of participants to remain within a preset tilt threshold where no feedback is provided to the user (deadzone).

As part of this study, participants stood on various surfaces (hard surface, single foam-covered surface and double foam-covered surface) with their eyes open or closed to simulate various types and degrees of balance dysfunction and determine the usefulness of vibrotactile feedback in patients with similar dysfunctions. For each combination of standing surface and eyes open or closed condition, a series of three 
tests were conducted; one with no feedback, one with feedback on the waist and one with feedback on the ankle. For each test, participants' trunk tilts in the AnteriorPosterior (AP) and Medial-Lateral (ML) directions with respect to a target position set at the beginning of each test were recorded and, in instances where feedback was provided, used to control the feedback provided to participants.

Using the data recorded during these tests, the best location for vibrotactile feedback was determined by comparing the ankle and waist performance on the following evaluation criteria: RMS AP trunk tilt, RMS ML trunk tilt, percentage time spent in the deadzone, reaction time and dominant motion frequency. In addition, the overall effectiveness of vibrotactile feedback in improving balance measures was investigated using the same measures.

\subsection{Methods}

\subsubsection{Subjects}

12 healthy subjects (6 males and 6 females) with no identified neural damage, with ages ranging from 21 to 27 years (average 24.4 years, standard deviation 1.6 years), volunteered to participate in the experiment. All subjects read and signed a consent form, stating that they were well informed of the types of activities this study entailed and of the data's use in furthering this research. This study was conducted in

accordance with ethics clearance provided by Carleton University's Research Ethics Board (protocol clearance number 101290).

\subsubsection{Procedure}

Each participant received 12 minutes of training using feedback. During this training period, participants spent 4 minutes each standing on 3 surface types: hard surface, 
single-foam covered surface and double foam covered surface. Participants received 2 minutes of feedback on the ankle and 2 minutes of feedback on the waist for each standing surface. Participants were first instructed to get accustomed to the sensation of vibrotactile feedback and to practice discerning which tactor (within the 4 tactor array circling either the ankle or waist) was activated by repeatedly tilting out of the deadzone to activate tactors. Once participants were accustomed to the feedback, they were instructed to practice maintaining their balance using the feedback provided to them on the ankle and waist.

Once the training period was completed, each participant took part in the 6 Trials outlined in Table 5.1; each of these trials is based on experiments conducted in previously published articles [50] [47] [15] [43] [19] [41] [51]. Participants performed each of the Trials with no shoes and their feet together (to provide the same support base width in both the AP and ML directions thus making participants equally unstable in both directions). For each Trial, participants performed 3 tests, one for each feedback state (no, ankle and waist feedback), each 2 minutes in duration. During each test, participants tried to maintain the same balanced position and when feedback was provided, they tried to minimize the number of feedback activation occurrences. Between each Trial, participants were typically given 1 minute to rest, but additional resting time was given when requested. Therefore, there were 6 minutes of data recorded for each of the Trials, and a total of 36 minutes of recorded data per participant for all 6 trials combined.

Due to the length of the testing process, muscle fatigue and training effects were a concern [50]. To reduce these effects three mitigation strategies were employed. First, participants were allocated to one of 4 groups, outlined in Table 5.2, thereby preventing these effects from creating erroneous differences in performance across feedback types. Second, all three tests (no feedback, feedback on ankle and feedback on waist) for each Trial were performed in immediate succession so that participants' 
Table 5.1: Experimental Trials

\begin{tabular}{ll}
\hline Trial & Trial Conditions \\
\hline Trial 1 & eyes open on a hard surface \\
Trial 2 & eyes closed on a hard surface \\
Trial 3 & eyes open on a single-foam covered surface \\
Trial 4 & eyes closed on a single-foam covered surface \\
Trial 5 & eyes open on a double-foam covered surface \\
Trial 6 & eyes closed on a double-foam covered surface \\
\hline
\end{tabular}

fatigue and training were similar within Trials. Third, the order in which these Trials were performed was randomized for each participant so that these effects did not create biased differences in performance across Trials.

Table 5.2: Participant Groups

\begin{tabular}{ll}
\hline Group & Testing Order \\
\hline Group 1 & $\begin{array}{l}\text { performed the tests first with no feedback, then with feedback on the } \\
\text { ankle followed by feedback on the waist }\end{array}$ \\
Group 2 & $\begin{array}{l}\text { performed the tests first with no feedback, then with feedback on the } \\
\text { waist followed by feedback on the ankle } \\
\text { proup } 3\end{array}$ \\
performed the tests first with feedback on the ankle followed by \\
feedback on the waist and with no feedback \\
performed the tests first with feedback on the waist followed by \\
feedback on the ankle and with no feedback
\end{tabular}

\subsubsection{Experimental Set-Up}

Figure 5.1 shows the main components of the experimental set-up used in this study. The experimental set-up consists of two sets of 4 tactors (one for the left ankle providing unilateral feedback and one for the waist) shown as parts D and E in Figure 5.1. They are activated by the Arduino board (part B) when the trunk tilt with respect to a target position measured by an inertial motion unit (part A) exceeds a 
preset threshold. How each of these components is mounted on participants is shown in Figure 5.2. Tactors were placed on the ankle and waist as outlined in the previous chapter, only they were held in place using elastic bands as opposed to bandages. To ensure that participants could feel the posterior tilt feedback on the waist, the tactor placed on their lower-back was offset from the center of their back to avoid areas of large curvature and ensure that the tactor was held in contact with the participants' skin.

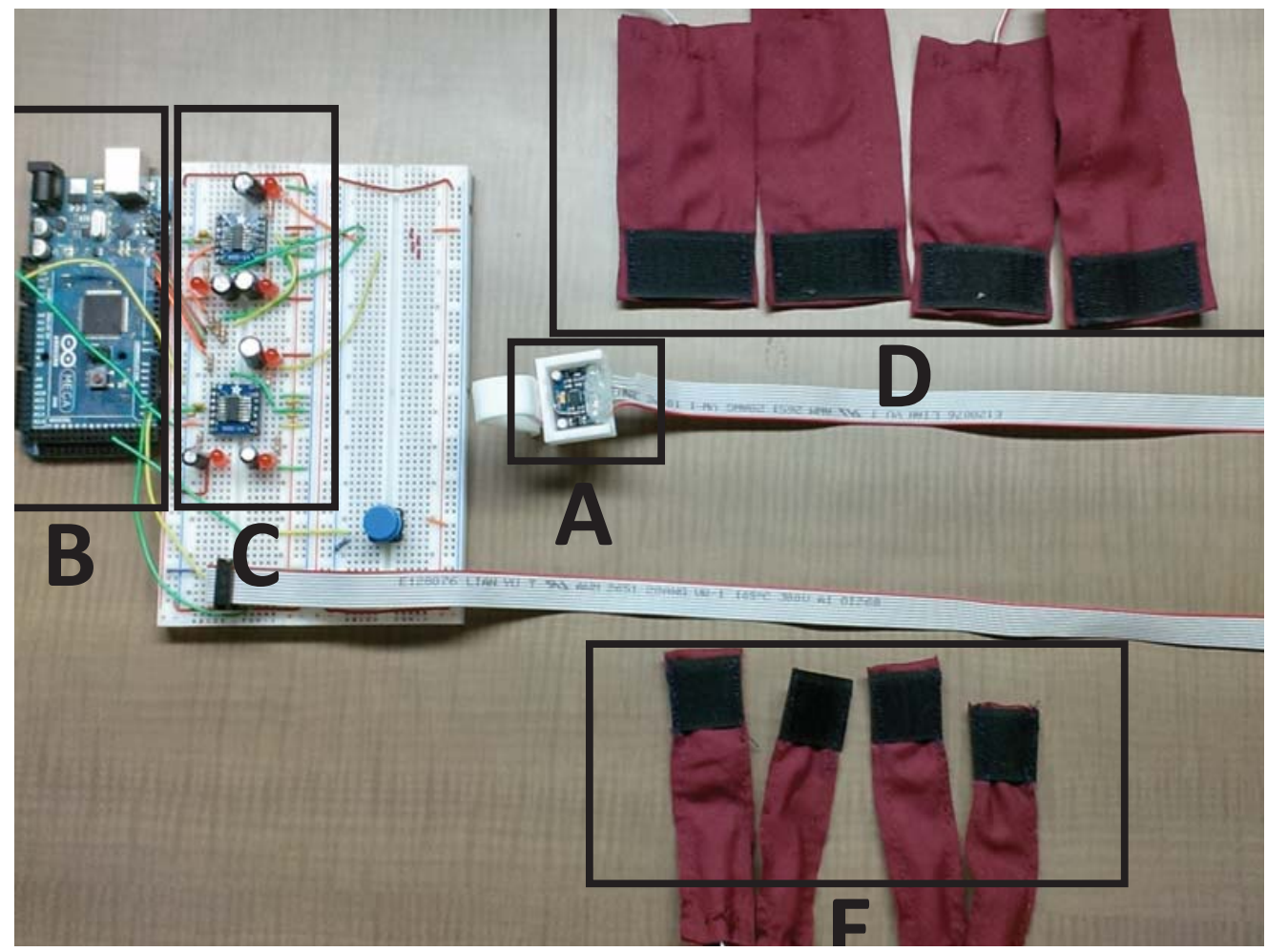

Figure 5.1: Experimental setup. The trunk tilt is sensed using the inertial motion unit (A) and recorded by the Arduino (B) for later analysis. The Arduino then identifies if feedback should be provided and sends the appropriate signal to the tactor driving circuits $(\mathrm{C})$ who activate either the tactors attached to the waist (D) or to the ankle (E) depending on the feedback condition (no, ankle or waist feedback). 


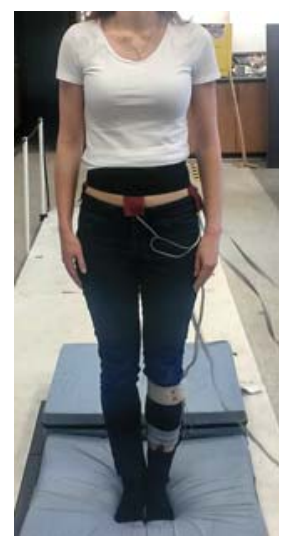

(a)

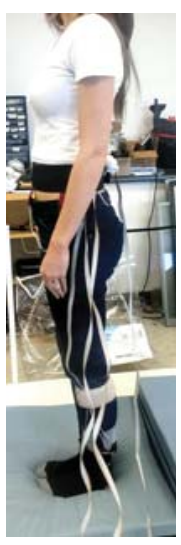

(b)

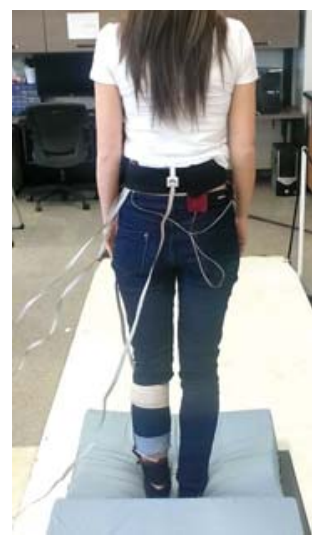

(c)

Figure 5.2: This figure demonstrates how each of the experimental circuit's components are worn by participants in this study through the use of a (a) front view, (b) side view and (c) back view.

\subsubsection{Tactor Attachment Bands}

To provide feedback on the waist, two belts were made to accommodate $90 \%$ of the population (based on American population anthropometric data, ranging from womens' fifth percentile score to mens' ninety-fifth percentile score), which meant they had to range in circumferences from $72 \mathrm{~cm}$ to $128.1 \mathrm{~cm}$ [52]. The smaller belt suited people with waist circumferences ranging from $72 \mathrm{~cm}$ to $96.5 \mathrm{~cm}$. The larger belt suited people with waist circumferences ranging from $96.5 \mathrm{~cm}$ to $130.2 \mathrm{~cm}$. One band was made to provide feedback on the ankle, based on the range of anthropometric measurements found in over $90 \%$ of the population (based on an anthropometric survey of 200 British Royal Air Force and Royal Navy aircrew, ranging from men's first percentile score to men's ninety-ninth percentile score); the circumferences of the lower leg immediately above the ankle bones ranges from $19.5 \mathrm{~cm}$ to $25.2 \mathrm{~cm}$, the dimensions accommodated by the ankle band [53]. 


\subsubsection{Angle Estimation Using Inertial Motion Unit (MPU6050)}

The MPU6050 was the inertial motion unit used in this experiment to estimate the trunk tilt in the anterior-posterior (AP) and medial-lateral (ML) directions. The MPU6050 was mounted on each participant's lower back for the duration of the experiment as shown in Figure 5.3.

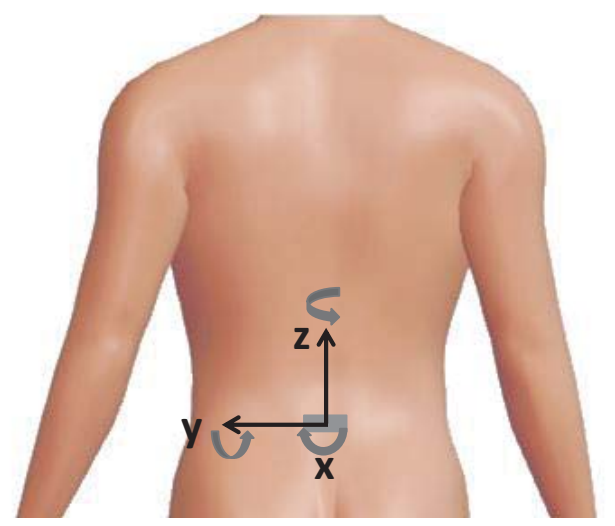

(a)

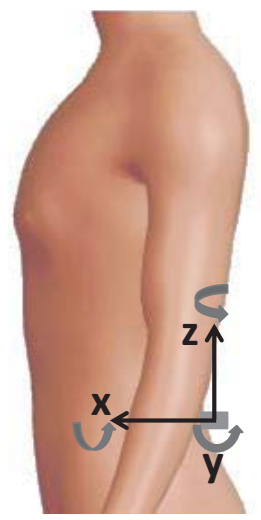

(b)

Figure 5.3: Orientation of the MPU6050 measurement axes with respect to participant trunk tilt during experiments. The positive acceleration axes are shown in black and the positive angular acceleration directions are shown in grey.

With the help of the MPU6050, the trunk tilt can be estimated using either the accelerometer or the gyroscope measurements. The gyroscope measurements can be used to calculate either the ML and AP trunk tilt, about the x or y axis, respectively, at some time $k$ by integrating the gyroscope's angular velocity measurements with respect to time as shown in Equation 5.1 [54].

$$
\theta_{g_{k}}=\theta_{g_{k-1}}+\omega_{g_{k}} * \Delta t
$$

The acceleration measurements from the 3-axis accelerometer can be combined to calculate instantaneous tilt about the $\mathrm{x}$ and $\mathrm{y}$ axes, based on Equations 5.2 and 5.3 [55] [56], respectively. These equations are created through modeling tilt rotations about the $\mathrm{x}, \mathrm{y}$ and $\mathrm{z}$ directions using Euler angles and composite rotation matrices. 
These composite rotation matrices were cross multiplied with the gravity vector to obtain a relationship between the Euler (in this case tilt) angles about a fixed coordinate system and the accelerations measured by the accelerometer in the $\mathrm{x}, \mathrm{y}$ and $\mathrm{z}$ directions [56].

$$
\begin{aligned}
& \theta_{x}=\arctan \left(\frac{a_{y}}{\sqrt{\left(a_{x}\right)^{2}+\left(a_{z}\right)^{2}}}\right) \\
& \theta_{y}=\arctan \left(\frac{a_{x}}{\sqrt{\left(a_{y}\right)^{2}+\left(a_{z}\right)^{2}}}\right)
\end{aligned}
$$

The trunk tilt used to determine if feedback should be provided to participants was the tilt angle away from a reference position. To establish this reference position, before each test, participants were asked to stand straight, feet together and eyes open on the surface on which the next test would be performed. The recorded reference vector then became the target position that participants aimed to maintain; AP and ML tilt angles were zero for this target position and feedback was calibrated based on the trunk tilt with respect to this target position. The target position was recorded on the surface on which testing would be conducted (e.g. if testing was on single foam, the target position was recorded while standing on single foam) because when moving from one surface to another, the trunk tilt corresponding to a balanced position varied significantly enough to affect the feedback provided to the participants and cause them to maintain awkward positions.

To estimate the angle with respect to the target position using the gyroscope, the tilt angle was set to have a start angle of zero degrees once the target position was set, to which the change in angle was added for each time increment, as already shown in Equation 5.1.

To estimate the angle with respect to the target position using the accelerometer, $\theta_{a}$, the tilt angle at each time point was calculated according to Equations 5.2 or 5.3, 
depending on the tilt direction of interest, and the difference between this angle and the corresponding angle measured for the target position represented the relative tilt angle estimate using the accelerometer, as shown in Equations 5.4 and 5.5.

$$
\begin{aligned}
& \theta_{a_{x}}=\theta_{x}-\theta_{\text {target }_{x}} \\
& \theta_{a_{y}}=\theta_{y}-\theta_{\text {target }_{y}}
\end{aligned}
$$

In order to improve on the tilt estimates made using the gyroscope or the accelerometer data, it is possible to combine the accelerometer and gyroscope data, more specifically $\theta_{a}$ and $\omega_{g}$, using a complimentary filter to provide a more accurate tilt angle estimate by retaining only the desired aspects of each measurement [57]. For instance, accelerometer tilt estimates have low drift, but very poor short-term estimation of tilt since they are affected by vibrations and impacts, and have a delayed response. Inversely, gyroscope tilt estimates exhibit considerable amounts of drift, but very accurately estimate tilt in the short-term [58].

The complimentary filter shown in Figure 5.4 fuses both sensors' data to calculate the combined estimate $\theta$. In this filter, the angular velocity measured by the gyroscope, $\omega_{g}$, is integrated using the integrator to obtain the gyroscope's tilt estimate , $\theta_{g}$, which is then filtered using the high pass filter. The tilt angle estimate calculated using the accelerometer data, $\theta_{a}$, is filtered using a complimentary low-pass filter. Low-pass filtering the accelerometer tilt estimate removes the high-frequency noise associated with vibrations or impacts while high-pass filtering the gyroscope's angle estimate removes the low frequency noise causing drift [57]. Therefore, the combined trunk tilt estimate is unaffected by each measurement system's noise [57].

From the block diagram shown in Figure 5.4, the following transfer function can 


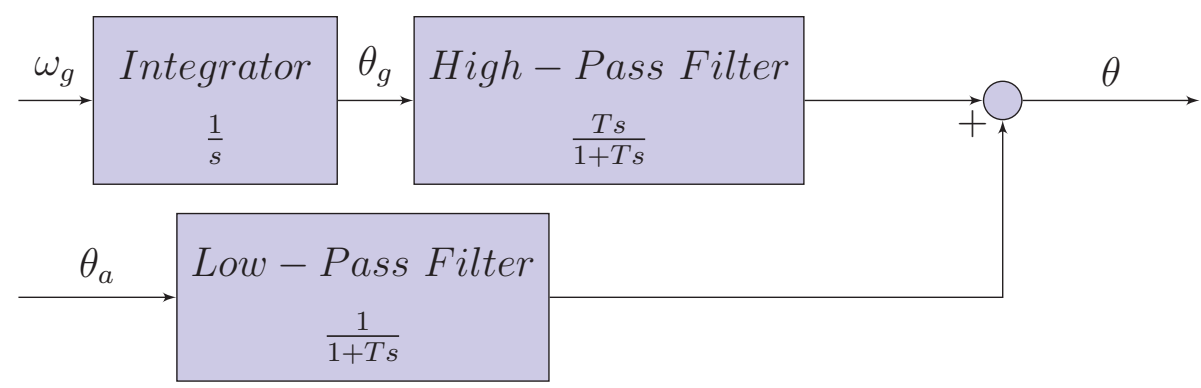

Figure 5.4: Block diagram of filter system used to fuse accelerometer and gyroscope estimates of the tilt angle $\theta$.

be found for the trunk tilt estimate based on the inputs to the system as [59]

$$
\theta=\frac{1}{1+T s} \theta_{a}+\frac{T s}{1+T s} \frac{1}{s} \omega_{g}=\frac{\theta_{a}+T \omega_{g}}{1+T s}
$$

where $\theta$ is the overall estimated trunk tilt angle, $\theta_{a}$ is the trunk tilt angle calculated using the accelerometer measurements, $\omega_{g}$ is the angular velocity measured using the gyroscope, and $\mathrm{T}$ is a constant that determines the filter cut-off frequencies $\left(T=1 / \omega_{c}\right.$, where $\omega_{c}$ is the cut-off frequency of the filters and is equal to $2 \pi f_{c}$, where $f_{c}$ is the cutoff frequency in $\mathrm{Hz}$ ).

As shown in [59], this transfer function can be transformed to an approximate form able to take as input discrete time measurements by using the z-transform and backwards difference method, according to which the following relationship holds:

$$
s=\frac{1-z^{-1}}{\Delta t}
$$

By substitution of Equation 5.7 into Equation 5.6, the following can be obtained:

$$
\theta=\frac{\theta_{a}+T \omega_{g}}{1+T \frac{1-z^{-1}}{\Delta t}}=\frac{\theta_{a} \Delta t+\Delta t T \omega_{g}}{\Delta t+T\left(1-z^{-1}\right)} .
$$

By cross-multiplication it can be found that

$$
\Delta t \theta+T\left(1-z^{-1}\right) \theta=\theta_{a} \Delta t+\Delta t T \omega_{g}
$$


where $\theta$ is in fact $\theta_{k}$ for some calculation of the $k^{\text {th }}$ value of the trunk tilt angle, and $z^{-1} \theta$ is $\theta_{k-1}$, the trunk tilt angle estimated in the previous time step. Therefore, Equation 5.9 can be transformed to include the present and previous angle estimates.

$$
(\Delta t+T) \theta_{k}-T \theta_{k-1}=\theta_{a_{k}} \Delta t+\Delta t T \omega_{g_{k}}
$$

Isolating for $\theta_{k}$, the following can be found:

$$
\theta_{k}=\frac{\Delta t \theta_{a_{k}}+\Delta t T \omega_{g_{k}}+T \theta_{k-1}}{\Delta t+T}=\frac{\Delta t}{\Delta t+T} \theta_{a_{k}}+\frac{T}{\Delta t+T}\left(\Delta t \omega_{g_{k}}+\theta_{k-1}\right) .
$$

Choosing $\alpha=\frac{T}{\Delta t+T}$ and $1-\alpha=\frac{\Delta t}{\Delta t+T}$, Equation 5.11 can be simplified to be: [59]

$$
\theta_{k}=(1-\alpha) \theta_{a_{k}}+\alpha\left(\Delta t \omega_{g_{k}}+\theta_{k-1}\right)
$$

Equation 5.12 was used to generate a program for the Arduino to estimate the trunk tilt that can be found in Appendix H. This approach was used for both the trunk tilt in the ML and AP directions, separately. The alpha value used in this instance was found empirically to perform best when equal to 0.98 , thereby yielding a $T$ value of

$$
T=\frac{\alpha \Delta t}{(1-\alpha)}=\frac{0.98 \Delta t}{0.02}
$$

The time between measurements was found to be experimentally approximately 0.0139 seconds, therefore $T=0.6811$. Using this $T$ value, the filter cutoff frequency for both the low and high pass filters can be found as

$$
f_{c}=\frac{1}{2 \pi T}=\frac{1}{2 \pi 0.6811}=0.2337 \mathrm{~Hz}
$$

It was found experimentally that over the span of a test (2 minutes) where the 
MPU6050 was kept stationary the estimated tilt values in both the AP and ML directions drifted by less than 0.05 degree on average and fluctuated by up to 0.1 degrees from the mean tilt, therefore justifying the use of this inertial motion unit in conjunction with tilt limits of 1 degrees, more than 10 times the error in measurement inherent in the test set-up.

\subsubsection{Tactor Activation}

The same tactor driving circuits and tactors (Precision Microdrives' 310-003) as outlined in the previous chapter were used to provide feedback to participants in this study. Feedback was provided to participants at two intensities, the low intensity corresponding to $0.9 \mathrm{~V}$ (the typical start-up voltage) across the tactor and the high intensity corresponding to $1.7 \mathrm{~V}$ across the tactor (just below the max voltage for the tactors) [46].

As was done in multiple previous studies, a deadzone (zone where no feedback was provided to participants) about the target position was implemented, along with a low-feedback and a high feedback region. Although the deadzone size varies across studies, it is common, especially in studies where the participants are healthy, to provide no feedback for tilt values deviating up to 1 degree from the target position, thus the deadzone in this study was for AP and ML trunk tilts of magnitudes up to 1 degree [18] [21] [6] [60] [17].

Similarly to the deadzone threshold, the threshold separating the low and high feedback zones varied widely across studies; of the studies using a 1 degree tilt deadzone, two ( [18] [61]) provided low feedback from 1 to 3 degrees of tilt, whereas two other studies ( [21] [6]) used limits varying from one patient to another. For simplicity and to reduce test length, the fixed boundary approach was used in this study, hence participants received low intensity feedback for tilt magnitudes greater than 1 and up to 3 degrees away from the target position, and high intensity feedback for tilt 
magnitudes greater than 3 degrees as shown in Figure 5.5 [18] [61].

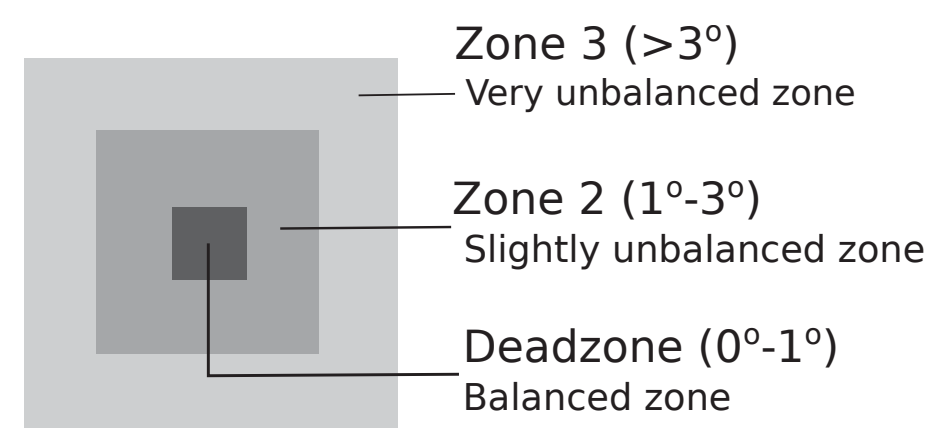

Figure 5.5: These are the feedback zones used in the experiment, the Deadzone (balanced zone) corresponds to tilt angles in the ML and AP direction of 0 to 1 degree, Zone 2 (slightly unbalanced zone) corresponds to tilt angles ranging from 1 to 3 degrees and Zone 3 (very unbalanced zone) corresponds to tilt angles greater than 3 degrees.

To allow users to more easily interpret feedback and reduce confusion effects on reaction time, only one tactor at a time would vibrate and feedback was provided for the direction (AP or ML) whose tilt magnitude was greatest. Furthermore, feedback in this study was repulsive, meaning participants were to move away from the direction in which they received feedback (e.g. if a vibration was sensed on the participant's left side, they should tilt to the right to become more balanced). Repulsive feedback was chosen since it is much more commonly used in balance improvement applications than attractive feedback (moving towards vibrations) and the goal of this study is to compare the effectiveness of feedback locations and types used in most studies. In addition, although participants have shown a natural tendency to move towards vibration feedback, this phenomenon is only observed for certain tactor locations (internal obliques and erector spinae) whereas other locations show no significant movement either towards or away from vibrations [7].

\subsubsection{Foam Surface}

The DREAMZONE Fold-A-Bed from JYSK was selected as the foam surface used for testing since it offers 3 panels 25 inches wide by 27 inches long and 3.6 inches 
thick with a foam density of approximately $23 \mathrm{~kg} / \mathrm{m}^{3}$. This foam was large enough to perform standing tests on, and had similar thickness and density as the foam used in a previous study (4 inches thick, density of $25 \mathrm{~kg} / \mathrm{m}^{3}$ ) [50].

\subsubsection{Data Analysis}

For each participant the RMS AP and ML trunk tilt, percentage time spent in the deadzone, reaction time and dominant motion frequency were calculated. The data for individual participants was then combined to calculate the mean and standard deviations for each of the aforementioned measures for each test. Six repeated measures ANOVAs with a Greenhouse-Geisser correction were conducted (one per Trial) using each measure's data pertaining to the Trial of interest and for which each feedback condition (no, ankle or waist feedback) was considered as the within-subjects factor. Using this test it was possible to identify whether a statistically significant $(\mathrm{p}<0.05)$ difference existed between two or more feedback conditions for each measure but not which two feedback conditions differed significantly. To determine the significance of pairwise comparisons, post hoc testing using the paired-samples t-test Sidak correction for multiple comparisons was conducted, where once again a p value smaller than 0.05 indicated a significant difference. In doing so, it was possible to ascertain whether feedback did improve participant balance and whether the feedback type made a difference in participants' performance.

Differences in participant performance when receiving no feedback versus when receiving feedback have different meanings depending on the Trial and its simulated balance dysfunctions. For instance, differences in performance with and without feedback for Trial 1 will help determine if healthy participants can benefit from biofeedback to improve their balance measures, whereas differences in performance with and without feedback for Trial 2 determine the effect of feedback for visually impaired patients. Trial 3 measure the effectiveness of feedback for patients with proprioceptive 
sensing impairments and Trial 4 determine the effectiveness for patients with both visual and proprioceptive sensing impairments. Trials 5 and 6 are similar to Trials 3 and 4, respectively, but simulate greater proprioceptive sensation loss.

The minimal sample size was calculated by using the $\mathrm{G}^{*}$ Power 3.1.9.2 software [62] based on an effect size of $0.9, \mathrm{p}=0.05$ and statistical power of 0.8 as being 10 . Twelve participants were used in this study to ensure that each of the 4 testing groups had the same number of participants. The effect size was derived from existing studies' mean differences for reaction time, percentage time spent in the deadzone and RMS trunk tilt values [6] [63]. Unfortunately, no preexisting data on dominant motion frequency exists thus it is possible the results found for this variable could lack clinical significance.

\subsection{Results and Discussion}

\subsubsection{Swaypath Variation with Feedback}

The swaypaths for all participants and tests can be found in Appendix I. In future subsections, data for each swaypath is quantitatively analyzed through the use of measures such as RMS trunk tilt, reaction time and percentage time spent in the deadzone. However, there is still some information regarding specific or unanticipated behaviors that can be gained through the visual analysis of swaypaths. Figure 5.6 shows swaypaths that present interesting patterns. For instance Figure 5.6a shows the participant beginning the test in a position anterior and to the left of the deadzone, however instead of moving in a direct trajectory back to the deadzone, the participant moved in a large sweeping manner so as to discern which direction would stop the vibrotactile feedback. Such sweeping motions observed in swaypaths show either a poor understanding of the feedback's meaning and the necessary reactions to this feedback or the ineffectiveness of training, something that cannot be observed using 
the quantitative data. This type of sweeping motion, although only observed in a few tests, only presents itself for ankle feedback and not waist feedback, which may indicate an overall better understanding of waist feedback when little training is provided, thereby making it a more intuitive form of feedback.

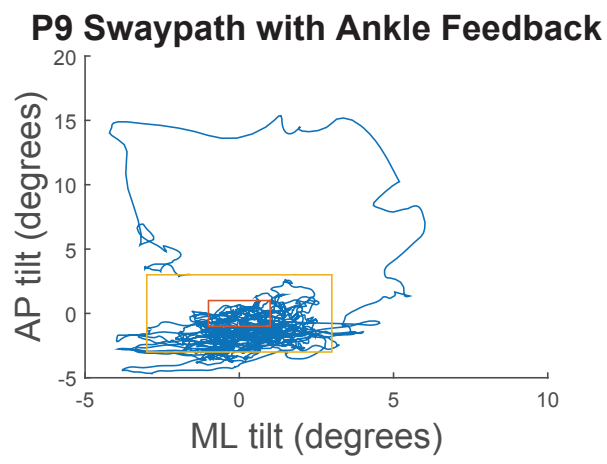

(a)
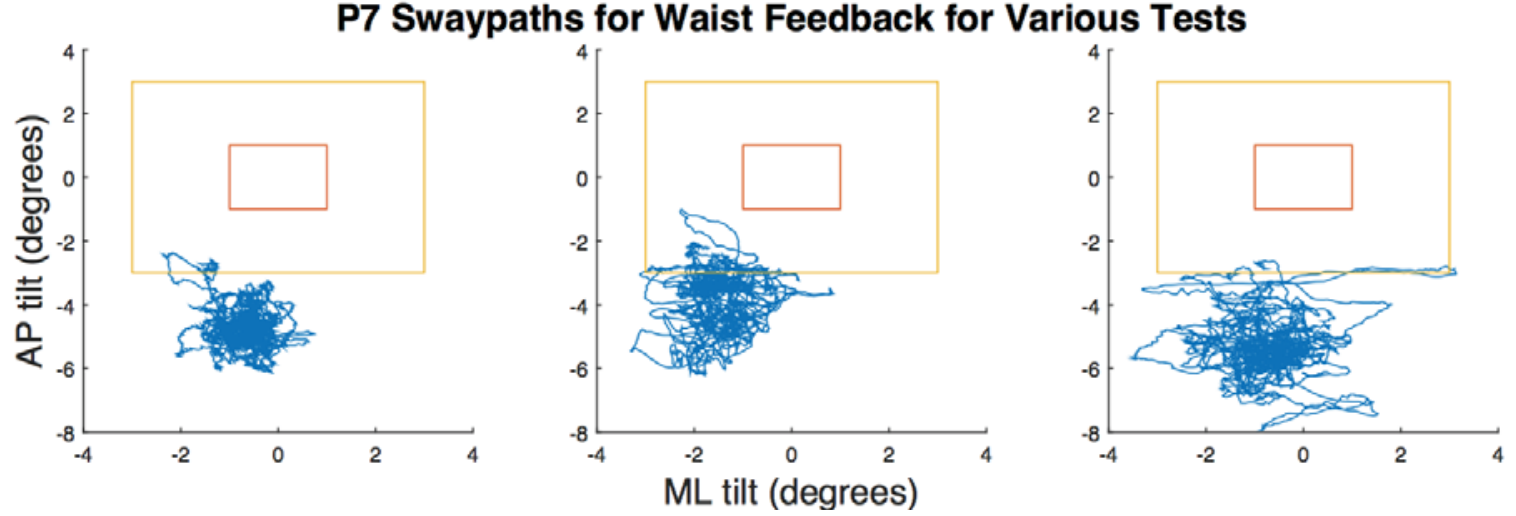

(b)

Figure 5.6: Sample swaypaths showing (a) either poor understanding or ineffective training for ankle feedback on double foam with eyes closed and (b) lack of sensitivity to feedback on the lower back for, left to right, standing eyes closed on a hard surface, eyes open on single-foam and eyes closed on single-foam.

Figure 5.6b shows that participant P7 did not feel or grew desensitized very quickly to feedback provided on his/her lower back, since tilt values oscillating around a point posterior to the deadzone were observed in all but one of this participant's tests where feedback was provided on the waist. This behavior was not present for tests performed with feedback on the ankle or no feedback, which means that this participant did not show a preference or tendency to tilt in the anterior position, thus these results for 
waist feedback were due to lack of sensation on the lower back. Although no other participant exhibited a similar behavior on all of their tests, some participants did mention they found the feedback on their lower back more difficult to sense than in other locations on their waist.

Furthermore, inspection of participants' swaypaths can even demonstrate the participants' natural disposition to have greater or smaller oscillation envelopes. For instance, as can be seen in Appendix I, P2 has a very small oscillation envelope of about 1 degree in radius for most tests, whereas P4 has a much larger oscillation envelope 4 degrees in radius for most tests. Therefore swaypath inspection could potentially be used as a predictor of a person's ability to to remain stable, or might even be used to tailor the boundaries for each feedback intensity to each individual.

\subsubsection{RMS AP and ML trunk tilt}

Overall, as seen in Figure 5.7, there is no sustained significant difference between the RMS trunk tilt in both the AP and ML directions when comparing ankle and waist feedback, inferring that they perform similarly when considering the RMS tilt. However some significant differences between the tests where no feedback is provided and where feedback is provided are present (results of repeated measures ANOVA and post hoc test found in Appendices $\mathrm{J}$ and $\mathrm{K}$ ). As determined through a repeated measures ANOVA using a Greenhouse-Geisser correstion, mean ML tilts differed statistically significantly in Trials 2 and 4 ( $\mathrm{p}=.008$ and .024 , respectively), whereas mean AP tilts differed statistically significantly in Trials 1,3 and $5(\mathrm{p}=.000, .024$ and .045 , respectively).

Post hoc testing using the Sidak correction revealed that although the mean AP tilt values for ankle and waist feedback are lower than for no feedback across all trials, only the differences exhibited in Trial 1 between feedback and no feedback cases are significant (ankle/no feedback $\mathrm{p}$ value $=0.003$ and waist $/$ no feedback $\mathrm{p}$ value $=$ 
0.001). Similarly, post hoc testing using the Sidak correction revealed that although the mean ML tilt values for ankle and waist feedback are lower than for no feedback for most trials, only the differences exhibited in Trial 2 between waist feedback and no feedback are significant $(\mathrm{p}$ value $=0.008)$. The fact that some Trials were identified as having significant differences through the repeated measures ANOVA, but through post hoc analysis were shown to no longer be significant could be due to a lack of statistical power. This should not be the case since trunk tilt effect size values were included in the calculation of the minimal sample size. However, these results still show that, although not consistently significantly, feedback does decrease trunk AP and ML tilt for people of varying abilities (either healthy or blind, no significant improvements were found for simulated deficiencies in proprioceptive feedback).

Contrary to expectations, there are no observable differences in mean tilt magnitude with assumed trial difficulty; the tilt magnitudes for the eyes closed on doublefoam trial (considered the most difficult trial) shows no clearly observable difference from other, believed to be easier, tests, especially in the AP direction.

Surprisingly, the hard surface (Trials 1 and 2) was the only surface for which significant improvements on AP trunk tilt were observed for both types of feedback; this demonstrates that even healthy participants can benefit from vibrotactile feedback to improve their RMS tilt. This sustained level of significant difference in RMS tilt may be due to the smaller inter-participant variations in RMS tilt, which enable smaller differences to be found as significant.

Interestingly, the standard error bars for AP tilt provided using waist feedback in Trials 2, 3, 4 and 5 were very large and overlapped with the horizontal axis. This may be due to the fact that a few people could not feel vibrations on their lower back, which meant they spent the entire test in a very unbalanced state (as seen in Figure 5.6b) and thus had very large RMS tilt magnitudes for trials with feedback on the waist causing these large variations across participants. This may serve as 
an indication that feedback on the waist may not be useful for everyone, and that, at the very least for some people, feedback on the ankle might be preferred since inter-participant variations are smaller for this feedback location.

Large variations in ML tilt are less common, the only critical variation value is observed for no feedback on double foam. Large variations in tilt when no feedback is provided are less concerning, and mostly have to do with the fact that many participants would spend the entire test outside the deadzone because they were unable to sense whether or not they were in it. This is especially true in cases where participant's natural balancing abilities were hindered on purpose, as is the case for the double foam trials. 


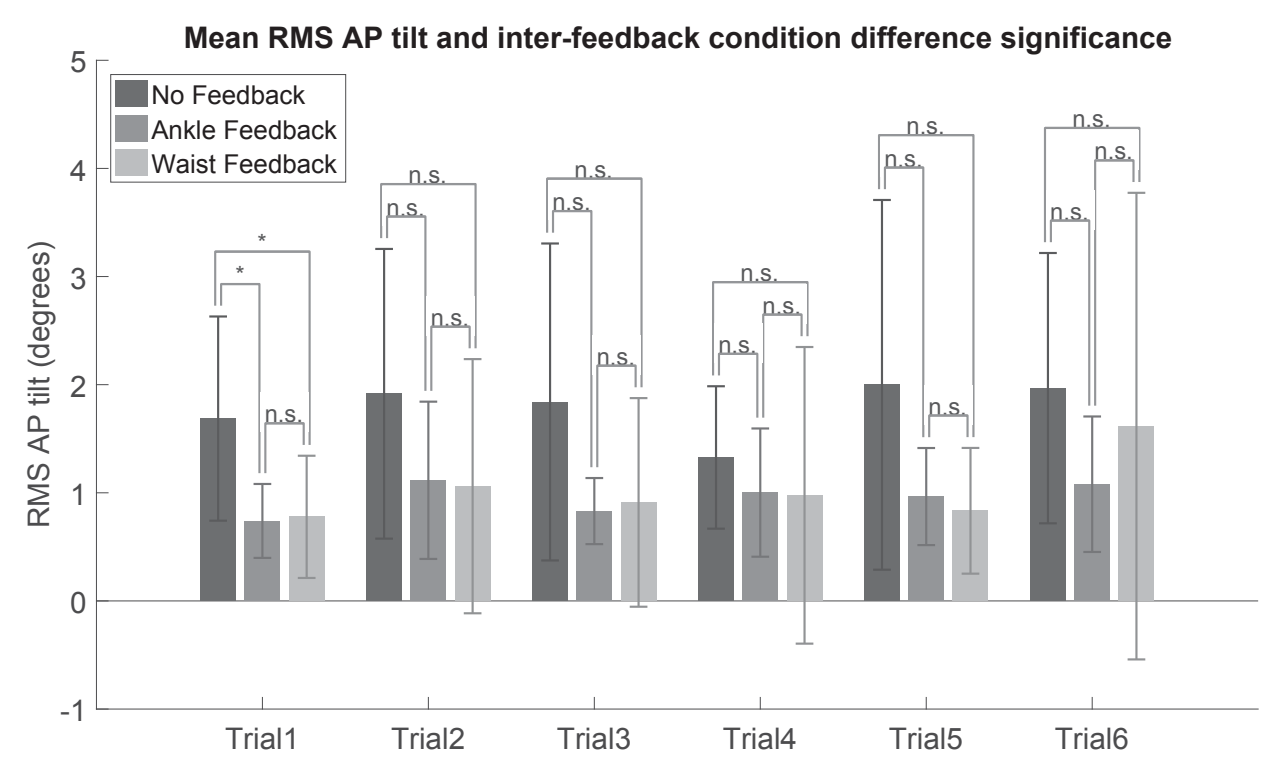

(a) mean and SD RMS AP trunk tilt

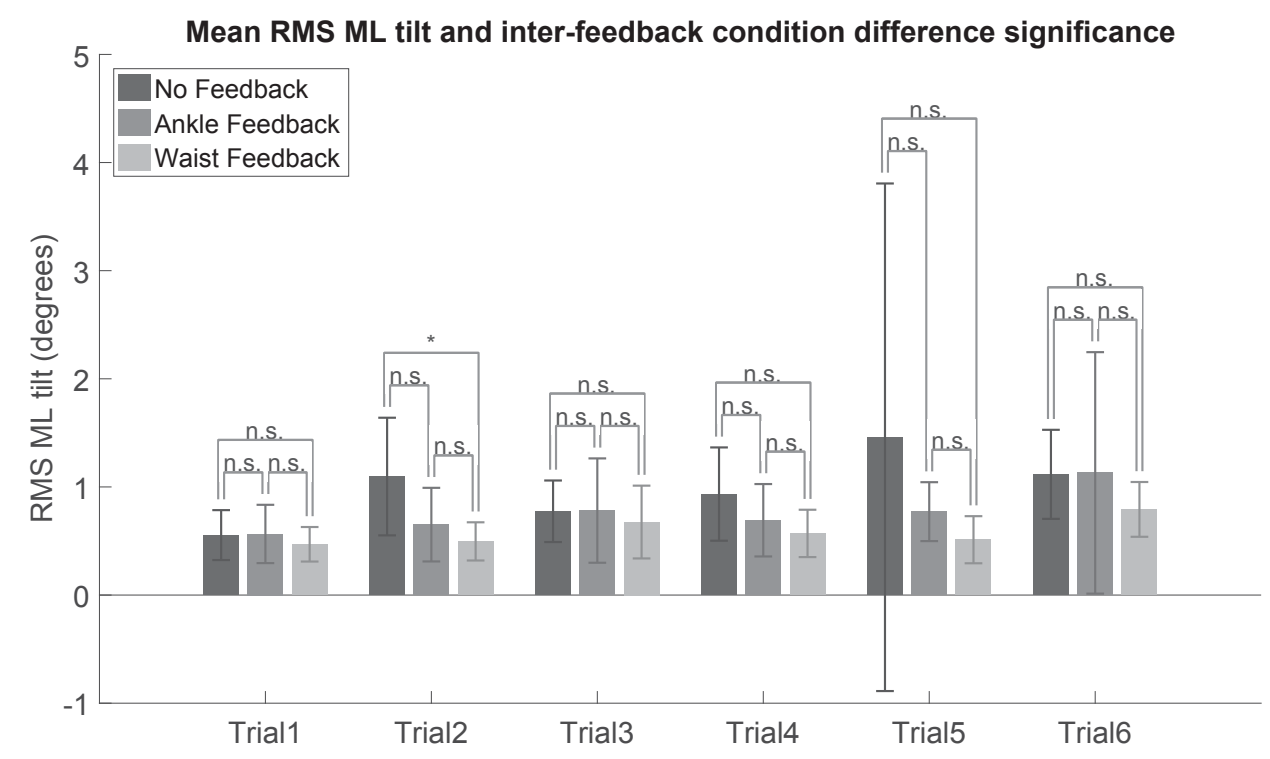

(b) mean and SD RMS ML trunk tilt

Figure 5.7: These are the mean values for the RMS AP and ML tilt for each Trial and each feedback condition. Feedback conditions' differences are indicated as not significant (n.s.) or significant with $\mathrm{p}<0.05\left(^{*}\right)$. The mean, SD and p values are all reported in Appendix K, Tables K.1 and K.2. 


\subsubsection{Dominant Movement Frequency}

The dominant movement oscillation frequency for each trial and each feedback condition (no, ankle or waist feedback) was found to further understand the participants' motion dynamics during each test. Should the movement frequency increase as the RMS tilt magnitude decreases, this could mean that the movement speed when seeking to remain in the deadzone is greater for the ankle and waist feedback conditions than when no feedback is provided. This could imply a different type of balance dysfunction whereby the participants' increases in motion speed within a smaller envelope could lead to instability while still providing improved tilt metrics.

As was observed in Figures 5.7a and 5.7b, the mean RMS sway for the ankle and waist are consistently smaller, although not always significantly so, than the no feedback tilt. Considering Figure 5.8 demonstrates no similar trend and no significant difference between any of the tests' dominant movement frequency, the participants' movement frequency is similar across tests for any of the trials. This would imply that motion should, on average, be of the same if not slower speed for the ankle and waist feedback tests than for the no feedback test. This supports the idea that as the mean RMS tilt decreases, the participants grow increasingly balanced.

There is however a noticeable difference between participants' dominant movement frequency for the double-foam eyes-closed trial (Trial 6) as compared to the other trials; the movement frequency is much greater for Trial 6. Considering Trial 6 also exhibited greater trunk tilt magnitudes than other trials in both the AP and ML directions (shown in Figure 5.7), this means that participant motion speeds are greater for Trial 6 than other trials and would seem to indicate a decrease in participant stability. These decreases in participant stability are present across all three feedback conditions therefore suggesting that they are related to the testing condition (doublefoam eyes-closed). The fact that this trial seems to perform very differently from 
other trials may be an indication that participants struggled to maintain balance not because they lacked the ability to sense their balance state, but because they were physically unable to maintain their balance due to the greater strength and endurance required to do so on double-foam.

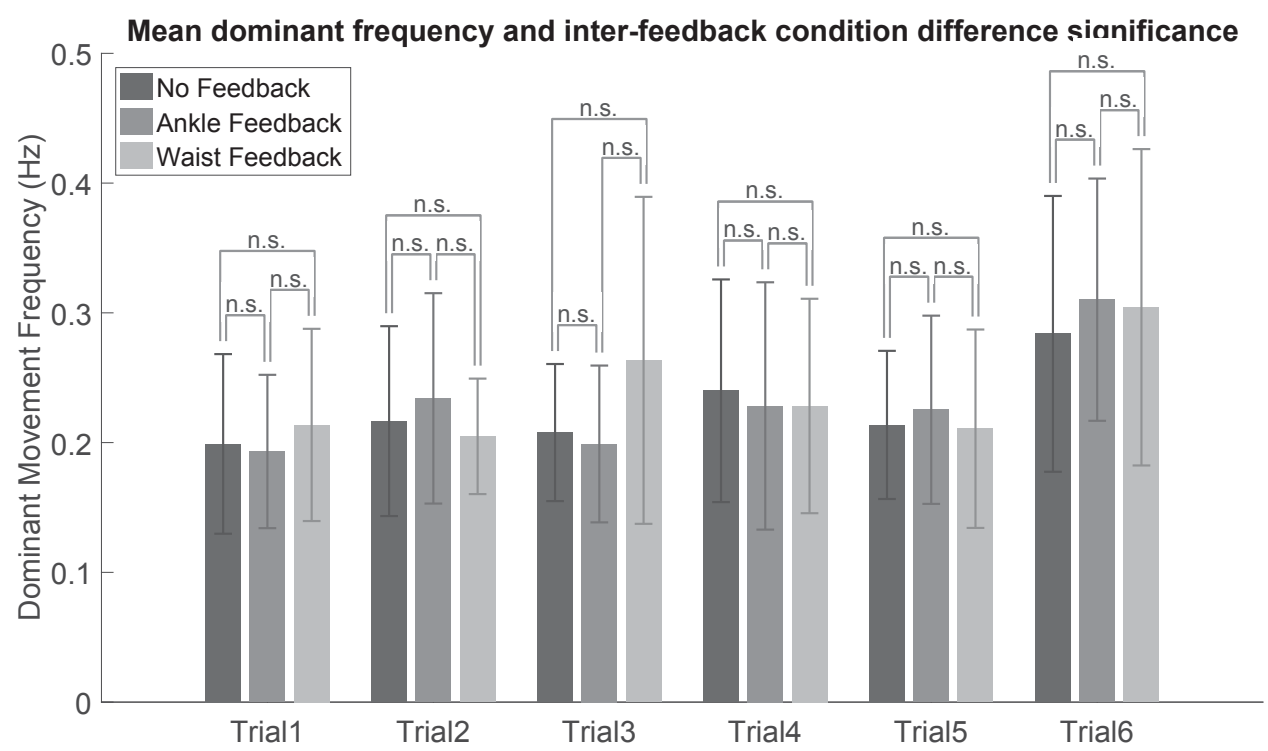

Figure 5.8: These are the mean values and the associated error bars for the dominant movement frequency for each Trial with no feedback or feedback on the ankle or waist. Feedback conditions' differences are indicated as not significant (n.s.) or significant with $\mathrm{p}<0.05(*)$.

\subsubsection{Reaction Time}

Figure 5.9 demonstrates the mean and standard deviations for each feedback condition during each Trial, as well as the level of mean difference significance between each feedback condition. As part of this study, the reaction time was found as the time difference between a departure from the deadzone event (feedback turns on) and a return to the deadzone event (feedback turns off). The number of reaction times recorded per test per participant varied based on the amount of times the participant strayed from the deadzone. The overall mean and standard deviation were found for 
each test by grouping each participant's mean reaction time in an array and finding this array's mean and standard deviation and can be found in Appendix K.

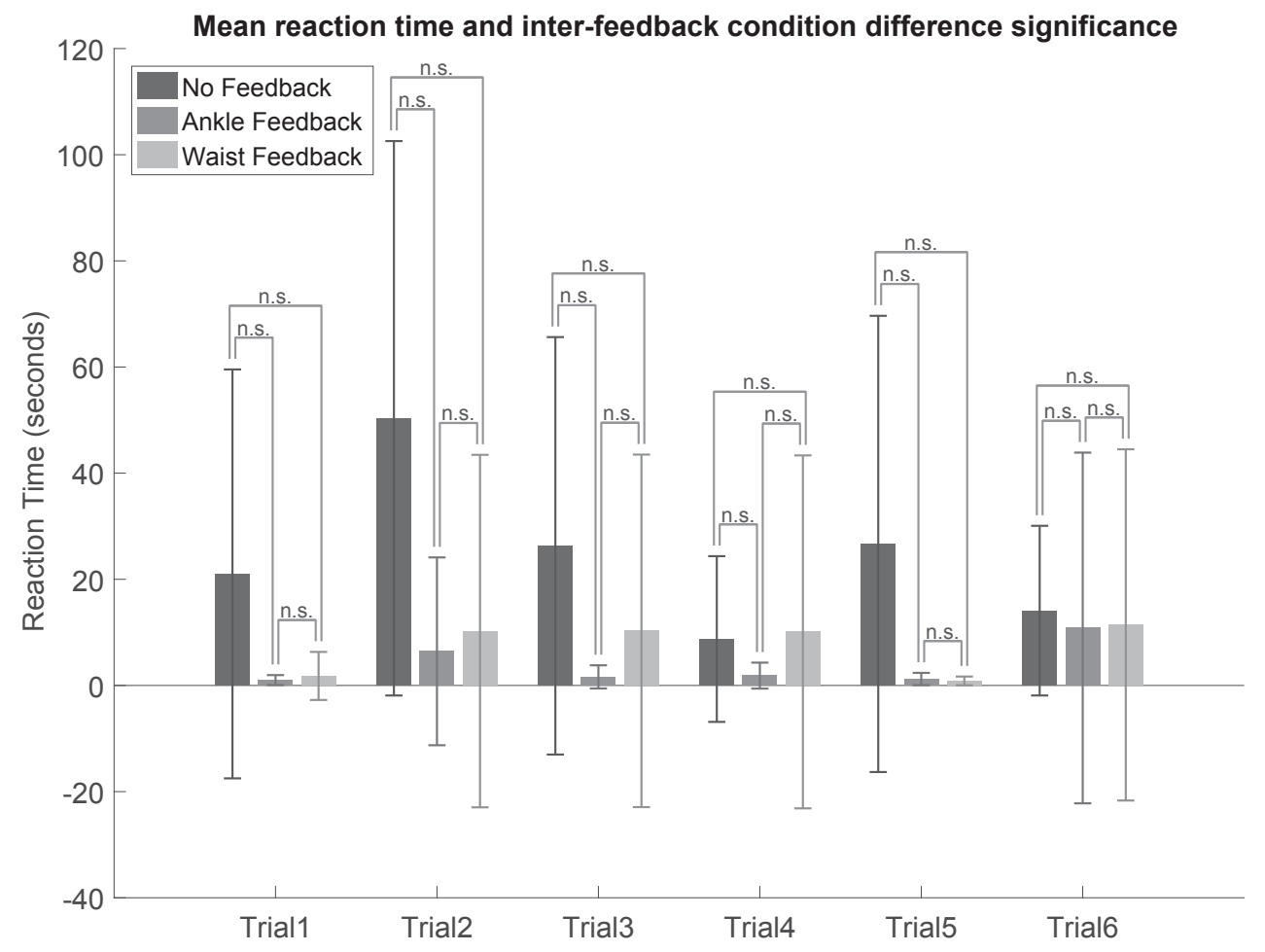

Figure 5.9: These are the mean values and the associated error bars for the reaction time for each Trial with no feedback or feedback on the ankle or waist. Feedback conditions' differences are indicated as not significant (n.s.) or significant with $\left.\mathrm{p}<0.05{ }^{*}\right)$. The mean, SD and $\mathrm{p}$ values are all reported in Appendix K, Table K.4.

The results in Figure 5.9 show large, although not significant, decreases in reaction times when feedback on the ankle or waist is provided as compared to when no feedback is provided for all trials conducted. Even though these decreases are in some cases very large (mean differences up to 43.9 seconds), the large variations between participants prevent these differences from being statistically significantly different. It is still possible that healthy, blind and people affected with diminished proprioceptive sensing could all improve their reaction time by using this type of vibrotactile feedback, however these improvements may not be significant. 
Once again, ankle and waist feedback have statistically similar reaction times, showing that one method is no more effective than the other at providing vibrotactile feedback on balance measures.

As could be expected, for each Trial the standard deviations for the no feedback cases are much larger than for the feedback cases. These large ranges in standard deviations show how participants' natural abilities to remain within the deadzone can vary greatly. However, when feedback is provided on the ankle, the reaction time is not only decreased but in most cases the performance across participants is increasingly similar regardless of participants' natural balancing abilities, thereby reducing standard deviations. This effect is not observed for waist feedback, perhaps because, as aforementioned, some participants did not sense feedback on their back and would spend the majority of the test outside the deadzone thereby exhibiting much larger reaction times than other participants. Nonetheless, the standard deviation ranges are much larger than the mean reaction times, regardless of the feedback condition, once again due to the fact that some people would spend very long periods of time outside the deadzone, sometimes an entire test length, which would make for large variations in reaction times across participants.

The reaction times observed in this study are somewhat comparable to those observed in other vibrotactile balance feedback studies. For instance, one study [17] found mean values ranging from 0.5 to 1.5 seconds, where the reaction times were measured in a similar manner to this study by calculating the time it takes participants to reach a deadzone (up to 1 degree in tilt away from a target position) and turn off the feedback, thus similar values are to be expected. The ankle feedback performed similarly to the feedback used in [17] for all the eyes open conditions (values ranging from 0.08 to 1.215 seconds), however the performance when the eyes were closed was over a greater range of values (1.854 to 10.83 seconds) which was no longer comparable to other studies. This indicates that at least one participant had added 
difficulty in keeping balanced when his or her eyes were closed, which lead to greater reaction times. The waist exhibited much greater, although not significantly different, mean reaction times than the ankle, often hovering around 10 seconds. These greater mean reaction times could once again be the result of one participant not sensing the feedback on their back and not trying to move towards the deadzone, causing a dramatic increase in the mean waist reaction time as opposed to the ankle reaction time.

\subsubsection{Percentage Time Spent in Deadzone}

The percentage time spent in the deadzone is a common measure often used in other studies [24] [60] [6] [64] [65] to demonstrate vibrotactile effectiveness in improving participants' balance state. In effect the percentage time spent in the deadzone calculates how much of the test length participants spend within the deadzone (or within 1 degree of the target position) receiving no feedback and are therefore considered to be balanced. Consequently, a greater percentage time spent in the deadzone indicates that participants spent a greater proportion of the test in a balanced state and is desired.

The repeated measures ANOVAs with Greenhouse-Geisser corrections identified that for every Trial mean percentage time spent in the deadzone differed statistically significantly between feedback conditions $(\mathrm{p}<0.005$ for all Trials). Through post hoc analysis with the Sidak correction, it was found that there are once again no statistical differences in the participants' percentage time spent in the deadzone when receiving ankle or waist feedback, for any of the trials, thus further demonstrating their interchangeability in feedback system design. However, statistically significant increases in percent time in the deadzone were observed through post hoc analysis of the repeated measures ANOVA output for all trials when comparing the performance of both types of feedback to that of the corresponding no feedback test ( $\mathrm{p}$ values are 
reported in Appendix K). This goes to demonstrate, based on the simulated balance dysfunctions, that both types of feedback have the potential to cause significant improvements to anyone's percentage time spent in the deadzone, regardless of their abilities.

The mean percentage time spent in the deadzone for the eyes open on a hard surface trial is nominally greater than for the other trials and the mean values for the eyes closed on a double foam are lower than for the other trials. However, the remainder of the trials show no differences to indicate the effects of the trial difficulty on the measure. The fact that no measure has shown any trend relating it to each trial's difficulty means that the stance task difficulty, analogous to the level of simulated disability, did not affect the results as much as the presence of feedback. In fact it could be said that the presence of feedback transcends the participants' inherent balancing abilities.

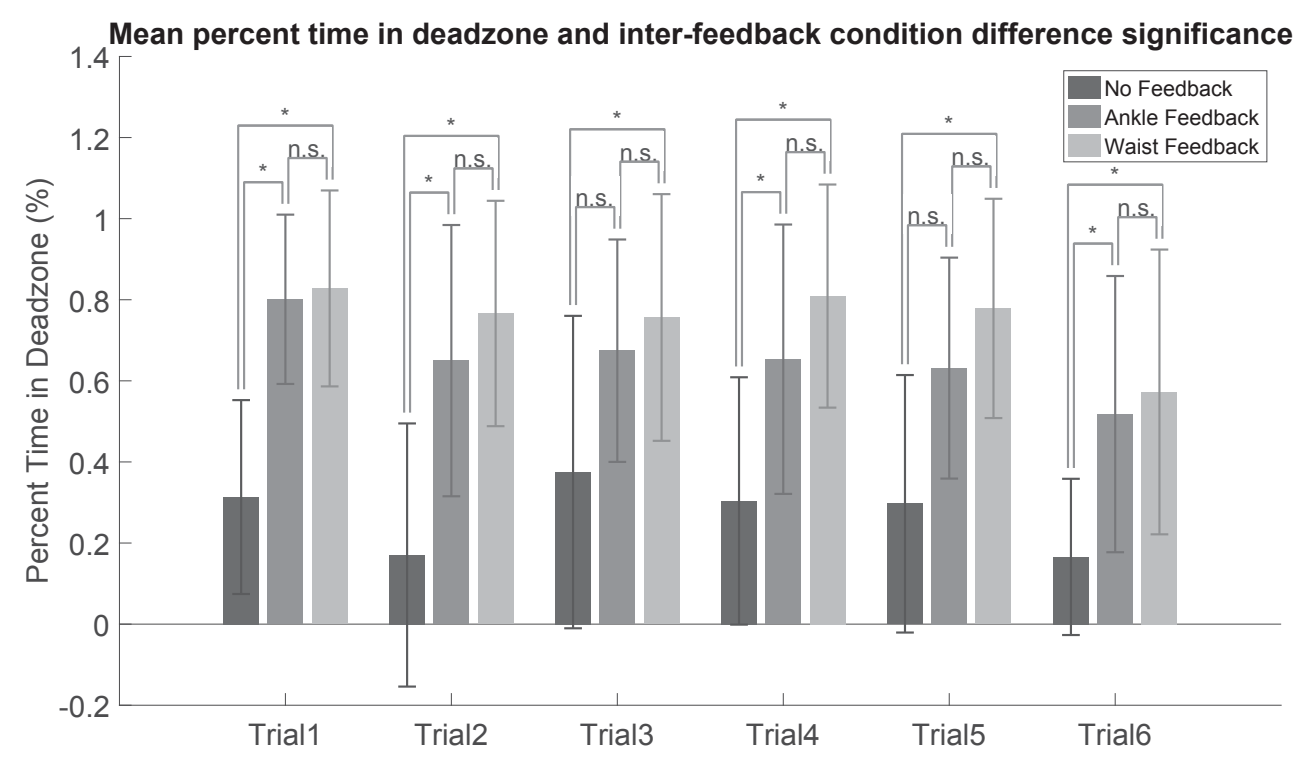

Figure 5.10: These are the mean values and the associated error bars for the percentage time spent in the deadzone (balanced) for each Trial with no feedback or feedback on the ankle or waist. Feedback conditions' differences are indicated as not significant (n.s.) or significant with $\mathrm{p}<0.05\left(^{*}\right)$. The mean, SD and $\mathrm{p}$ values are all reported in Appendix K, Table K.5. 
Lastly, only the no feedback conditions for Trials 2, 5 and 6 exhibit larger standard errors than their means which cause standard error bar overlap with the horizontal axis. This is once again due to the fact that participants would often spend large proportions of a test outside the deadzone when no feedback was provided because they were unable to determine whether or not they were in the deadzone.

\subsection{Summary}

It was found that vibrotactile feedback does improve balance measures; it significantly improved the the percentage time spent in the deadzone for all trials and the RMS trunk tilt in the AP and ML directions for some trials performed as part of this experiment. In addition, feedback on the ankle and waist both decreased, although not significantly, the mean reaction times for all trials, causing decreases in reaction time of up to 43.9 seconds.

Moreover, even though the RMS tilt, thus the tilt envelope, of participants decreased with feedback, the participants' oscillation movement frequency within their tilt envelope remained the same, indicating that movement speeds to maintain balance with feedback were either the same or slower than when no feedback was provided. This leads to the belief that not only are participants spending more time in a balanced state, but they are more stable while maintaining their balance when they receive vibrotactile feedback.

It was also found that the waist and the ankle perform statistically similarly across all balance measures and could therefore be used in balance improvement applications interchangeably. Arguably the ankle might be of greater use than the waist in applications where the balance measurement depends on the foot location instead of the trunk tilt since some participants have difficulty interpreting feedback when the balance measure is unrelated to the feedback location, as evidenced by 
the sweeping swaypath motions observed only for ankle feedback when the feedback information pertained to the waist. Moreover, it was shown that some participants did not sense the feedback on their lower back when waist feedback was provided, which once again makes the case for the use of ankle feedback in balance applications. 


\section{Chapter 6}

\section{Conclusions}

As part of this thesis, a comprehensive review of the literature on balance improvement using vibrotactile feedback was conducted. Through this review, a need for thorough comparison in vibrotactile feedback performance across various body sites was identified. To address this gap in research, three studies were designed, conducted and analyzed. The first study aimed to compare two tactor types used in other studies to further understand their vibration characteristics and determine which tactor type lends itself to improving vibrotactile feedback. The tactor type that best performed was then used in the second and third studies. The second study investigated participants' ability to sense and detect vibrotactile feedback on various body locations (neck, waist, wrist and ankle) and aimed to identify the top two performing locations by comparing weighted scores based on various measures (reaction time, ability to detect feedback, and ability to discern which tactor was activated at which intensity). The two locations that scored the highest were used in the third study. This last study consisted of testing the effect of vibrotactile feedback on balance by comparing participants' performance on balance measures (swaypaths, AP and ML tilt, dominant movement frequency, reaction time and percentage time spent in the deadzone) when feedback was located on the ankle and waist. It aimed to determine whether vibrotactile feedback on the waist and the ankle could significantly improve 
participants' balance and which feedback location produced greater improvements.

The main conclusions and contributions are highlighted below.

\subsection{Type of Tactor for Use in Future Studies}

As previously outlined, two types of tactors were considered in this work; the ERM and LRA. The ERM was found to be the more easily detected tactor type since its vibrations were sensed by participants in $100 \%$ of the cases, as compared to the LRA which was detected for less than $89 \%$ of the cases overall. For this reason, the ERM was used throughout the remainder of the studies. This increased performance in a tactor that vibrates at a frequency ranging from $75-125 \mathrm{~Hz}$, more than two times smaller than the commonly used vibrotactile feedback frequency of $250 \mathrm{~Hz}$ could imply that studies should select tactors based on the type of tactor actuation rather than the tactor's vibration frequency.

\subsection{Feedback Detection Predictor}

In Chapter 3, preliminary testing with 3 participants showed the acceleration and displacement amplitudes to be the effective predictors of participants' ability to sense feedback tested and exhibited specificity and sensitivity sums of 1.510 and 1.556 (correctly predicting $75.5 \%$ and $77.8 \%$ of cases), respectively.

In Chapter 4, further testing with a greater sample size of 10 participants showed that the acceleration and displacement amplitudes were not significant predictors of participant's ability to detect feedback. In fact, the sums of the specificity and sensitivity for these predictors were only slightly greater than 1 , meaning that feedback detection was correctly predicted in just over $50 \%$ of cases.

A metric which could be of interest in determining participant's ability to sense 
feedback, observed in Chapter 5, is the swaypath. Through examination of participants' swaypaths it would seem it is possible to identify whether they can feel certain tactors or even if they would require additional training with the device. Perhaps the use of swaypath analysis in predicting participants' understanding and ability to sense feedback could be formally investigated in future studies.

\subsection{Optimal Location for Vibrotactile Feedback}

In Chapter 4, a set-up was developed for users to communicate the feedback they perceived by pressing the button (within a button array) related to the location and vibration intensity of tactors in a tactor array. Through these tests, it was found that the waist was the best overall location for feedback due to its higher total score taking into account participants' reaction times, percentage detected feedback, percentage correctly identified tactor, and percentage correctly identified intensity. The ankle was a close second and performed statistically similarly to the waist across all evaluation metrics.

To further compare the waist and ankle locations, their performance on balance metrics (trunk tilt, reaction time, dominant movement frequency and percentage time spent in the deadzone) was tested in Chapter 5. This study aimed to determine which location would serve best in a balance improvement application. It was once again found that the ankle and waist performed statistically similarly. However, drawbacks are associated with each body location. For instance, feedback on the back of the waist was not felt by some participants which might mean that it is not a feasible solution for every patient. In contrast, feedback on the ankle was always felt, but for some participants elicited large sweeping motions when they left the deadzone. This was likely due to the fact they did not fully grasp the information the ankle feedback was trying to convey, which may mean that more thorough training is required for 
ankle feedback or that it is less intuitive than waist feedback.

\subsection{Vibrotactile Feedback Effect On Balance}

Lastly, it was shown in Chapter 5 that vibrotactile feedback does significantly improve some balance measures in its users. For instance, significant increases in participants' percentage time spent in the deadzone were observed for all Trials when comparing performance of ankle and waist feedback to no-feedback performance. Although not observed for all Trials, AP and ML tilt showed significant decreases when feedback was provided as opposed to when no feedback was provided for trials performed on a hard surface where participants had their eyes open and closed, respectively. Furthermore it was shown that participants not only reduce their tilt envelope when using vibrotactile feedback, but the movement speeds they exhibit are the same or slower than when they received no feedback, which seems to imply that participants are not only more balanced, but also more stable.

\subsection{Future Work}

\subsubsection{Improve Balance Measures}

Currently in the field of vibrotactile feedback use in balance improvement, one of the most prominent measures used to ascertain feedback effects on balance is trunk tilt. This measure is often determined through the use of an IMU and a reference vector as was done in Chapter 5. Nonetheless, through the use of this measure in Chapter 5's testing, trunk tilt measurements seemed to provide flawed identification of a person's balance state. For instance, changes in shoulder posture would often affect the tilt measured and cause participants to receive feedback even though their balance state did not change, only the curvature of their back did. This would often 
lead to participants maintaining an awkward position, sometimes less balanced than the position which elicited the feedback, for the remainder of the test. Therefore, a better balance measure needs to be developed, and ideally this measurement system would allow for dynamic identification of a participant's balance state to ensure that the feedback system is useful in as many occasions as possible.

For this reason, the Advanced Biomechatronics and Locomotion (ABL) lab is currently developing a system able to discern the wearer's balance state using CMP (Centroidal Moment Pivot) and ZMP (Zero Moment Point) measures, which take into consideration COP and foot placement. It is hoped that this measure of balance would provide a better representation of the user's actual balance state than the existing trunk tilt estimate which arguably acts as more of a posture measurement system.

\subsubsection{Build Integrated Balance Feedback System}

Since there are now two different systems in existence in the ABL lab; a balance detection system and a feedback system, the integration of both systems should be conducted in order to ensure for proper testing of the efficacy of the system in improving balance and preventing falls. The feedback system used in this study could be improved to produce a more commercial product and, as aforementioned, a new balance measure would need to be developed for this system. Since this feedback system will use balance measures unrelated to trunk tilt, it might be useful to once again compare the effectiveness of ankle and waist feedback in improving balance. It may be found that ankle feedback performs better than waist feedback when ankle position and orientation is more closely related to the balance measure. 


\subsubsection{Older Adult and Patient Testing}

Furthermore, all of the tests performed as part of this thesis were performed by healthy young adults, thereby demonstrating that even healthy participants can benefit from feedback. However, now that feedback has been shown to improve balance in the healthy young adult population, testing for the target populations (older adults and balance dysfunction patients) should be conducted. In fact the very tests performed in this study should be conducted once again with participants from these target populations. In addition, conducting the same tests with older adults could be helpful in distinguishing age effects on sensitivity to vibrotactile feedback.

\subsubsection{Improve Testing Protocol}

Through the administration of the tests in Chapter 5 some observations were made regarding potentially beneficial protocol changes for future tests on vibrotactile effectiveness on balance measures. For instance, the double-foam trials (Trials 5 and 6) should no longer be performed because they are believed to lead to greater participant instability due to their inability to physically maintain balance as opposed to their inability to sense their balance state. Regardless of the cause, healthy young adults struggled to maintain their balance during the double-foam trials, hence these tests should not be performed by older adults and/or balance dysfunction patients.

Furthermore, by the end of testing, participants often felt discomfort, and in some cases pain, due to extended standing. This could be mitigated by implementing breaks within trials (between the no, ankle and waist feedback tests) and increasing the length of breaks between trials. In fact, if the double foam trials were removed, participants' standing time would be reduced by 12 minutes. 


\subsubsection{Study Other Representations of Balance}

This study thoroughly investigated the effects of feedback on balance related measures for various types of static stance exercises. Now that the effects on static stance are known, further testing should be conducted to determine the effects of feedback during dynamic and complex exercises, since it is more likely that falls would occur during these types of events as opposed to static stance events. Future tests could therefore include walking trials (either with or without obstructions in the walking path or sitto-stand and stand-to-sit trials. These tests could also consider the effects of feedback

during secondary-task trials where participants are also focusing on another task and therefore have to give balancing themselves divided attention. 


\section{Appendix A}

\section{Meta-analysis results for the overall}

\section{biofeedback effect on AP tilt (regardless}

\section{of tactor/sensor location)}

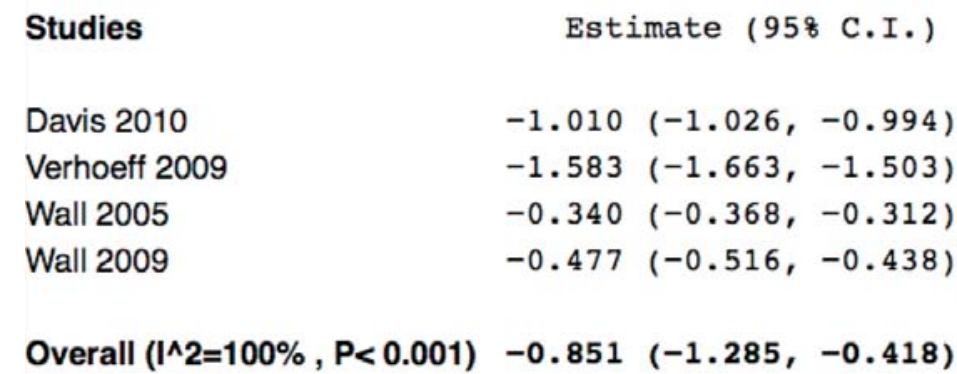

(a) Numerical summary of study results

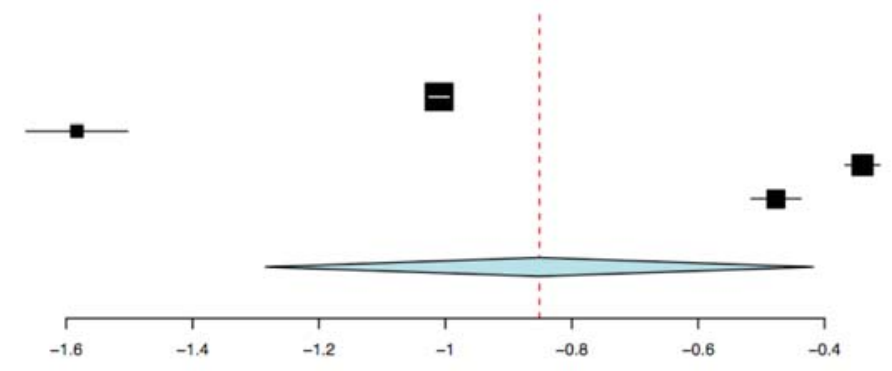

(b) Pictorial summary of study results 


\section{Appendix B}

\section{Meta-analysis results for the overall}

\section{biofeedback effect on ML tilt (regardless of tactor/sensor location)}

$\begin{array}{lrrr}\text { Studies } & \text { Estimate (958 C.I.) } \\ \text { Wall 2001 } & -0.106 & (-0.164,-0.048 \\ \text { Verhoeff 2009 } & -0.378 & (-0.442,-0.314 \\ \text { Davis 2010 } & -0.510 & (-0.529,-0.491 \\ & & & \\ \text { Overall (|^2=99\%, P<0.001) } & -0.332 & (-0.581, & -0.083\end{array}$

(a) Numerical summary of study results

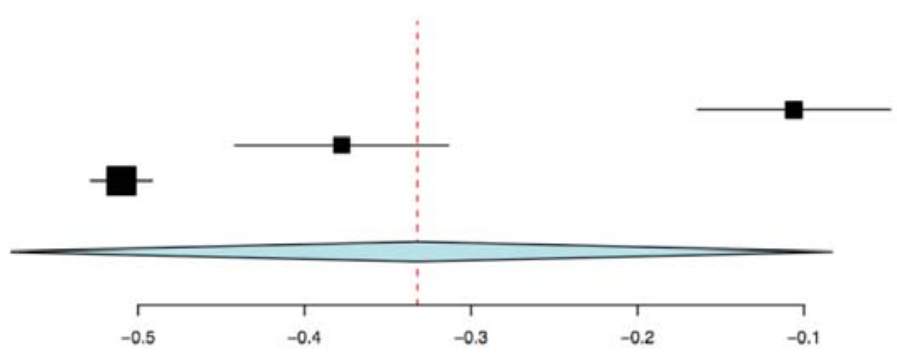

(b) Pictorial summary of study results 


\section{Appendix C}

\section{Minimum, Mean and Maximum Values}

\section{for the ERM and LRA Measures}

\section{C.1 Power Spectral Density as a Function of Fre-} quency for an ERM on Muscle Tissue

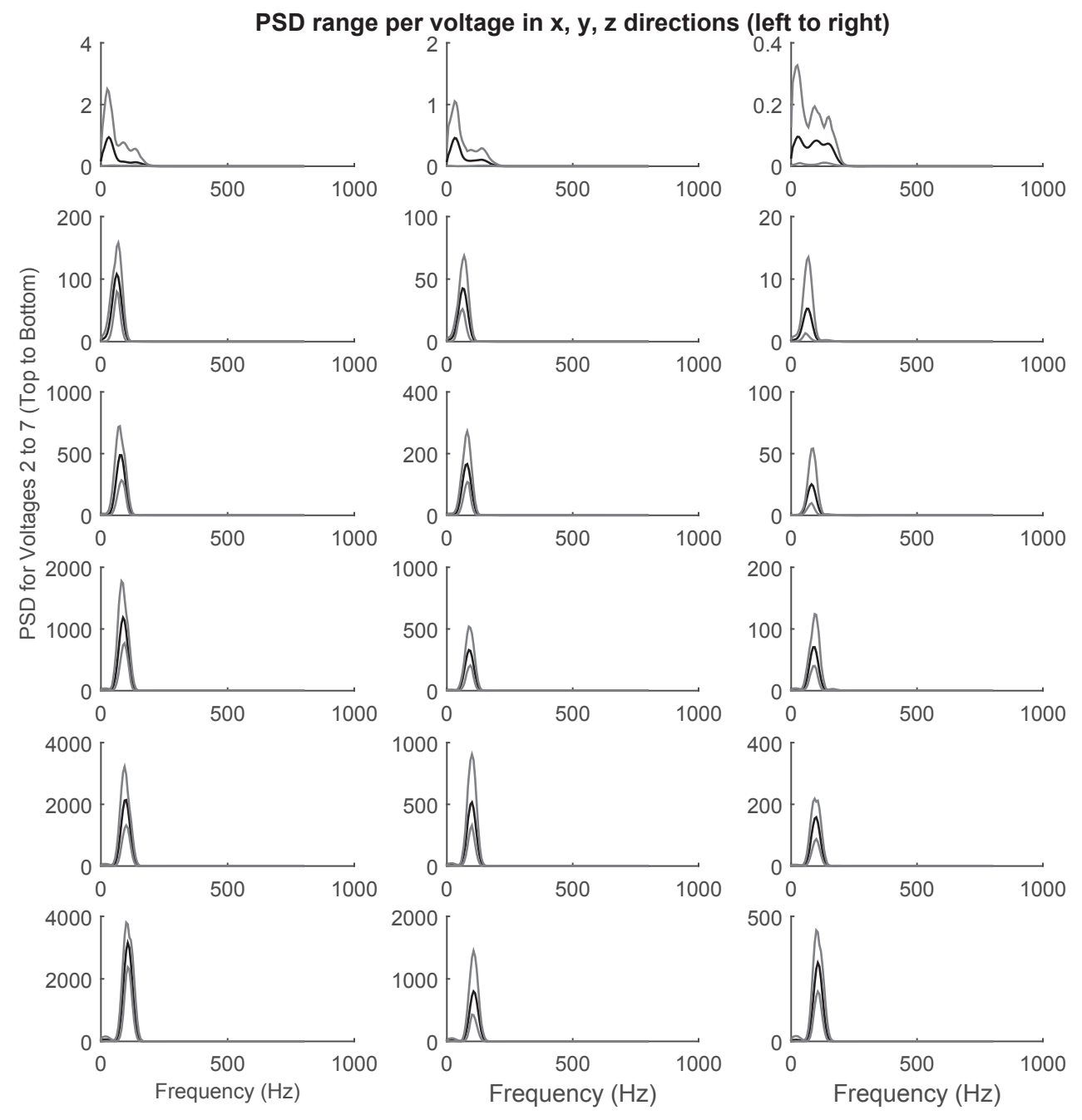




\section{C.2 Power Spectral Density as a Function of Fre- quency for an ERM on Bone Tissue}
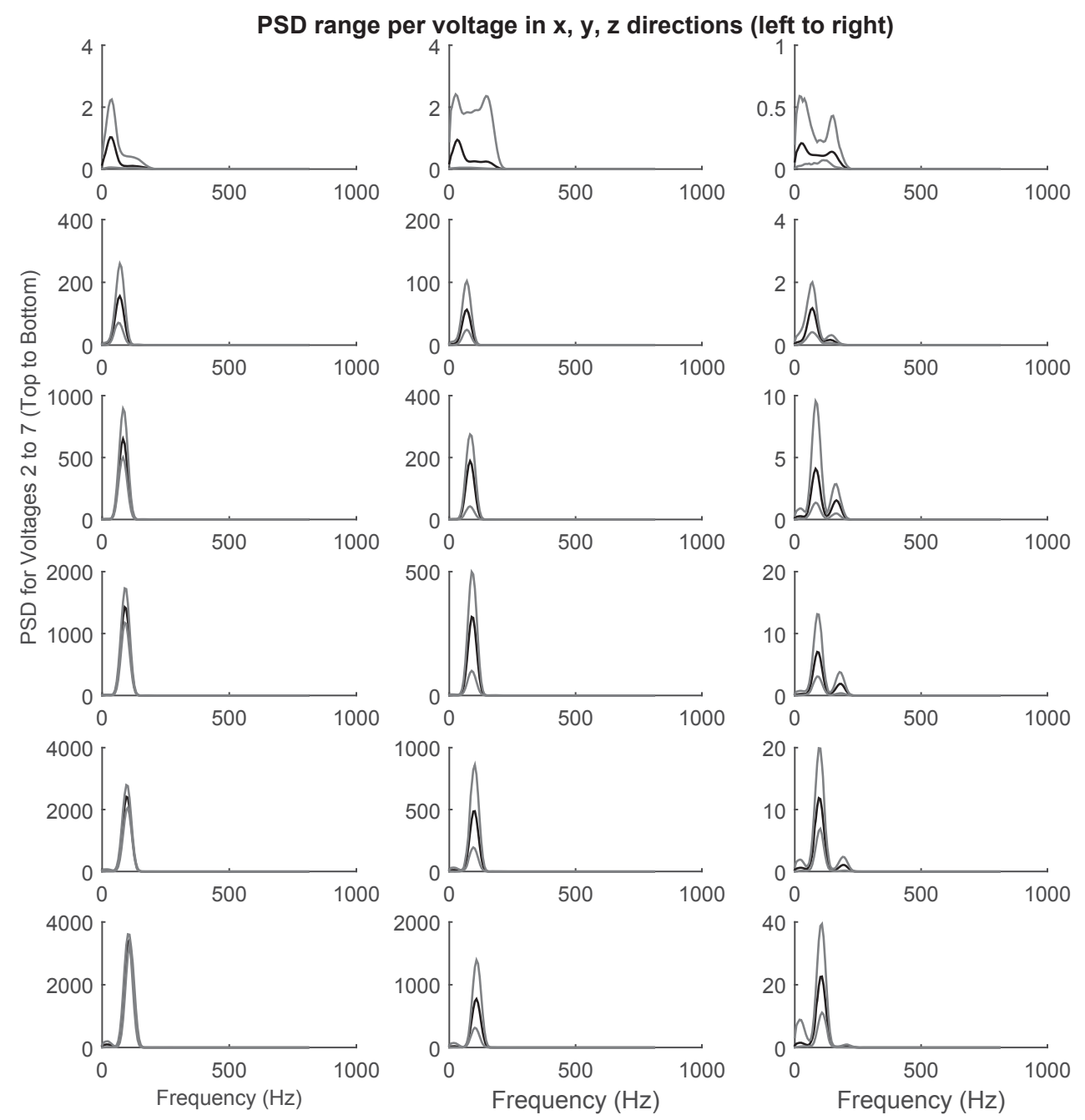


\section{C.3 Power Spectral Density as a Function of Fre- quency for an ERM on Lipid Tissue}
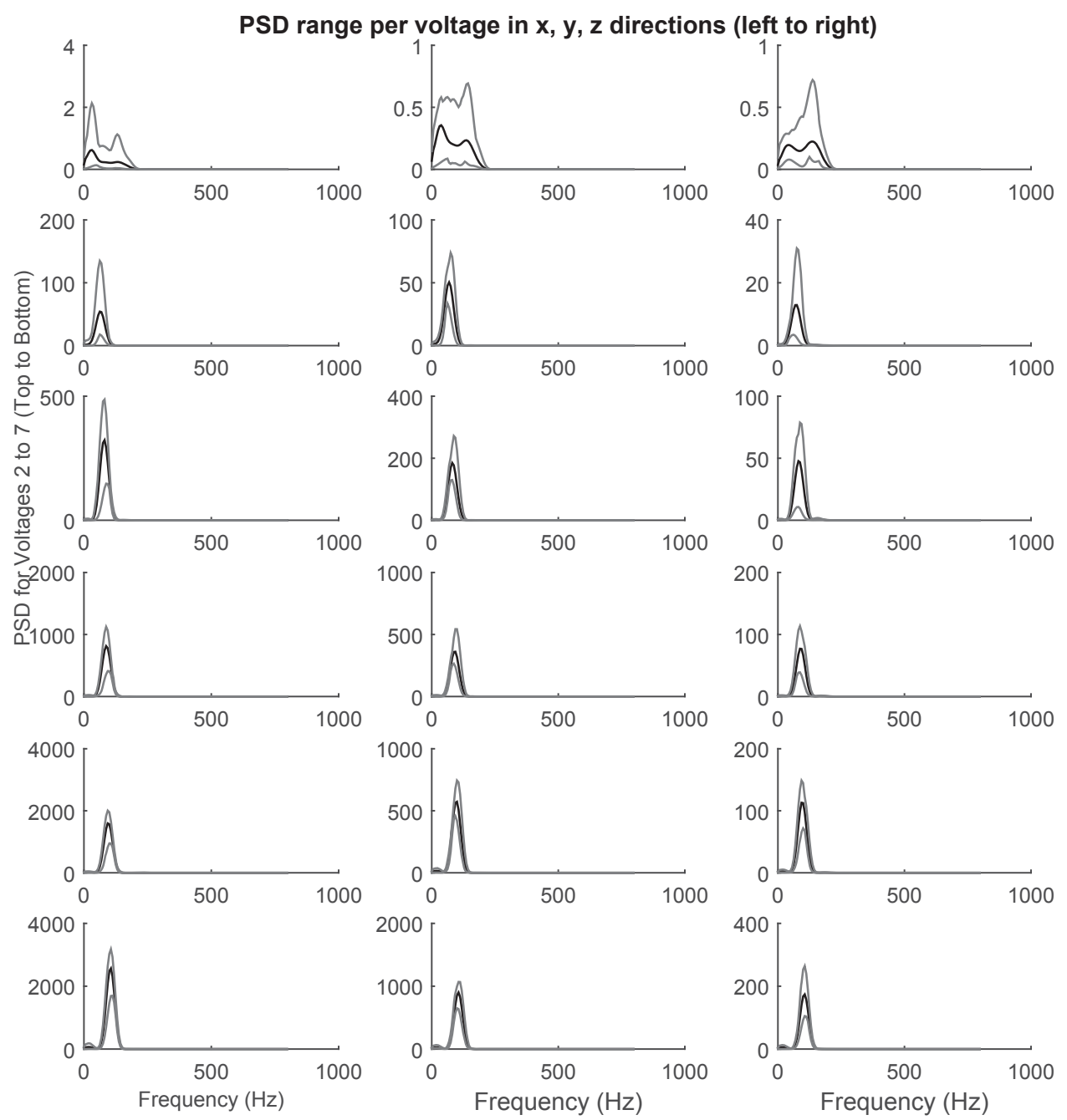


\section{C.4 Power Spectral Density as a Function of Fre- quency for an LRA on Muscle Tissue}
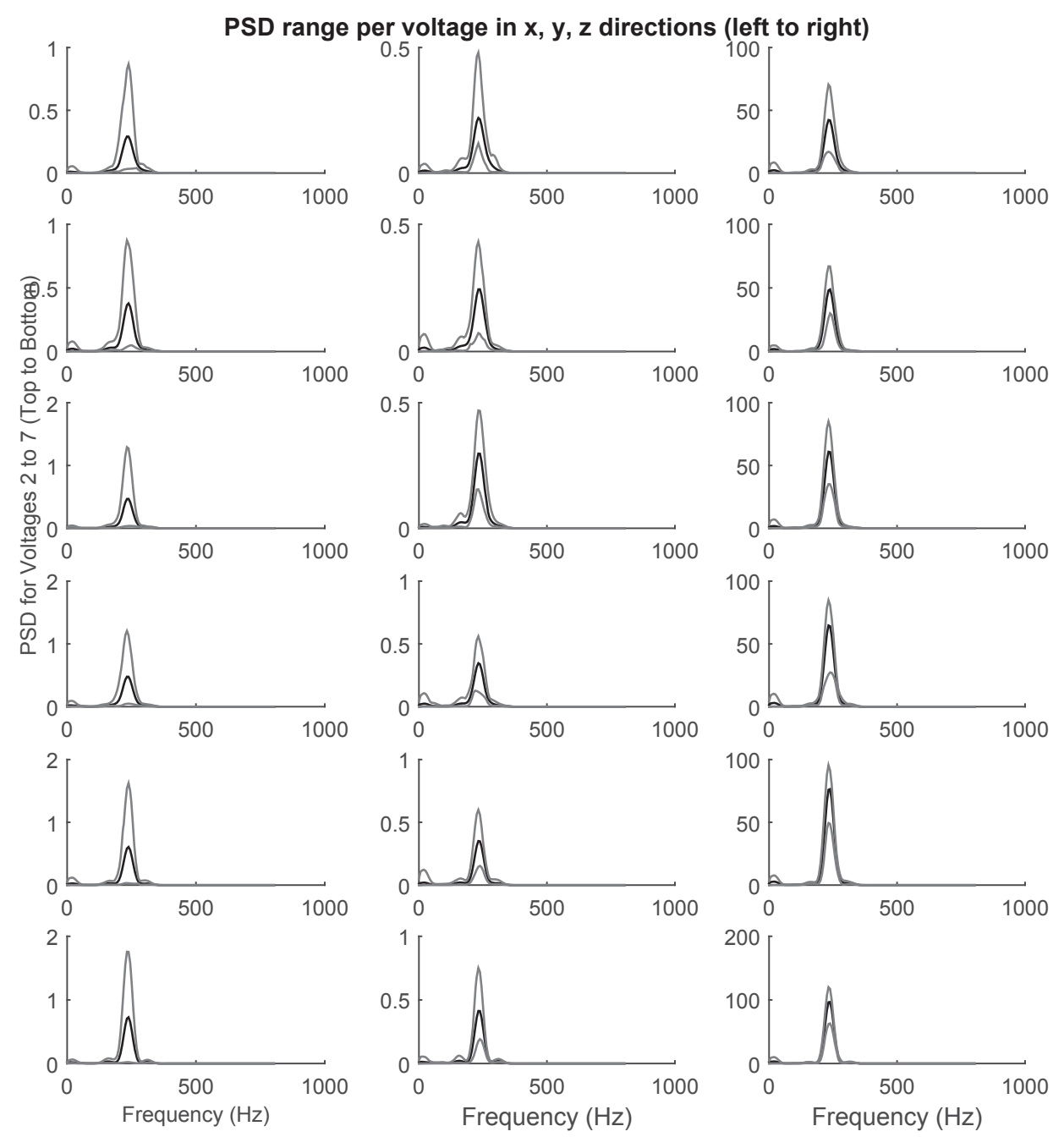


\section{C.5 Power Spectral Density as a Function of Fre- quency for an LRA on Bone Tissue}

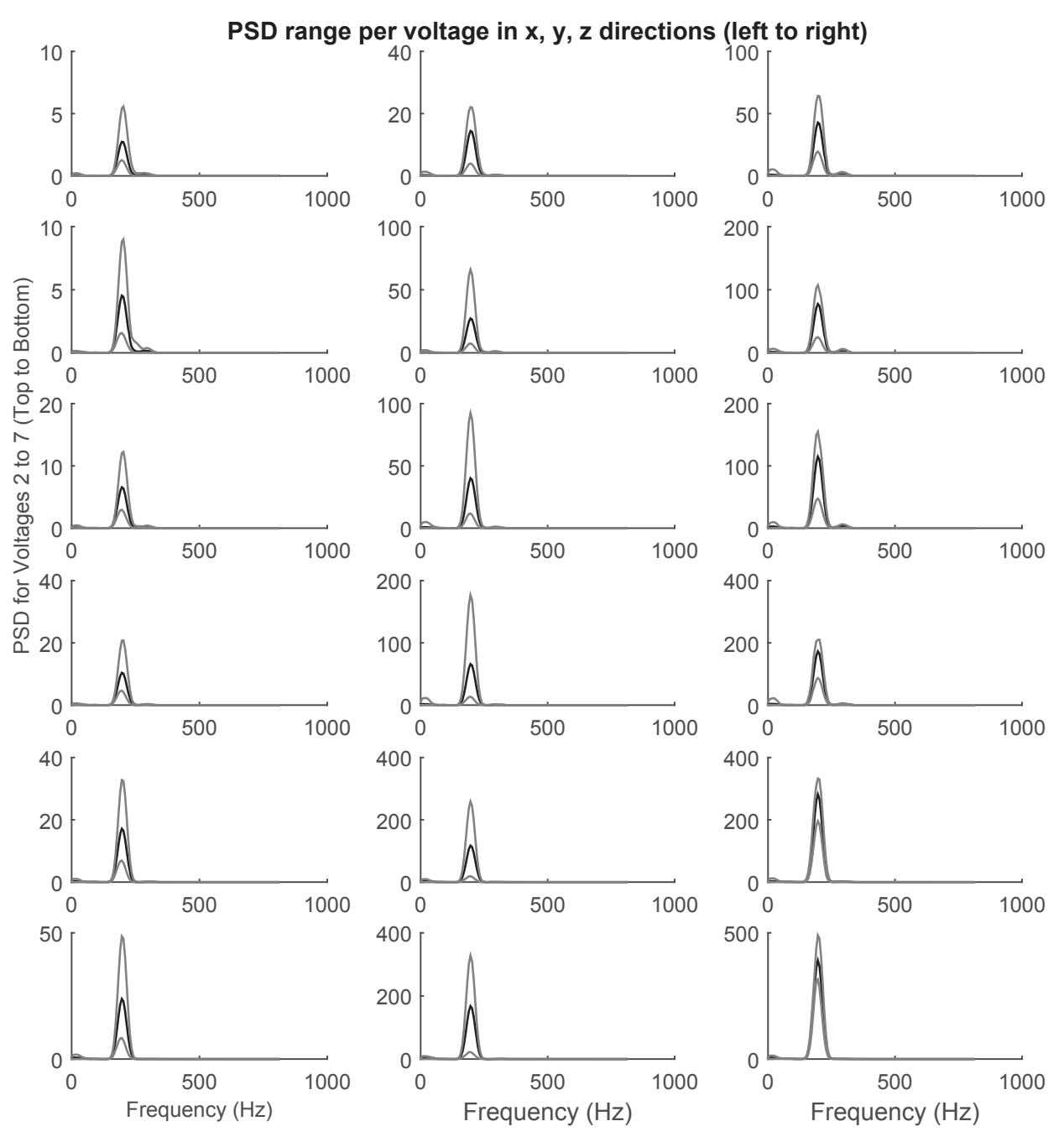




\section{C.6 Power Spectral Density as a Function of Fre- quency for an LRA on Lipid Tissue}
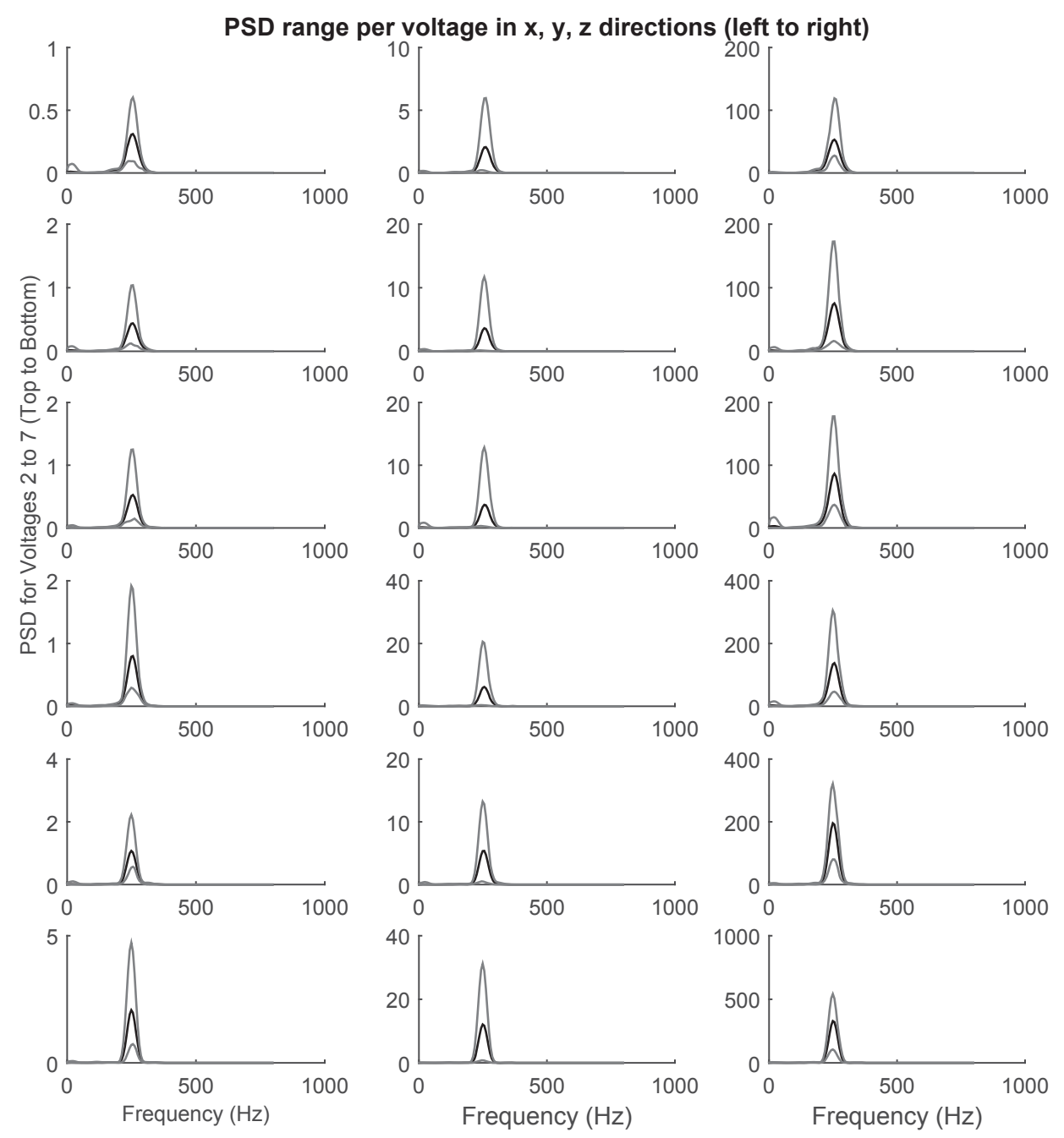


\section{Appendix D}

\section{Schematic Representation of the Test Circuit Connections for the Location Optimization study}

Figure D.1 shows the connections between each of the ribbon cables shown and labeled in Figure 4.2. The tactor driving circuits in Figure D.1 are essentially icons that replace the circuits shown in Figure 4.3 to allow the schematic to be clearer. 


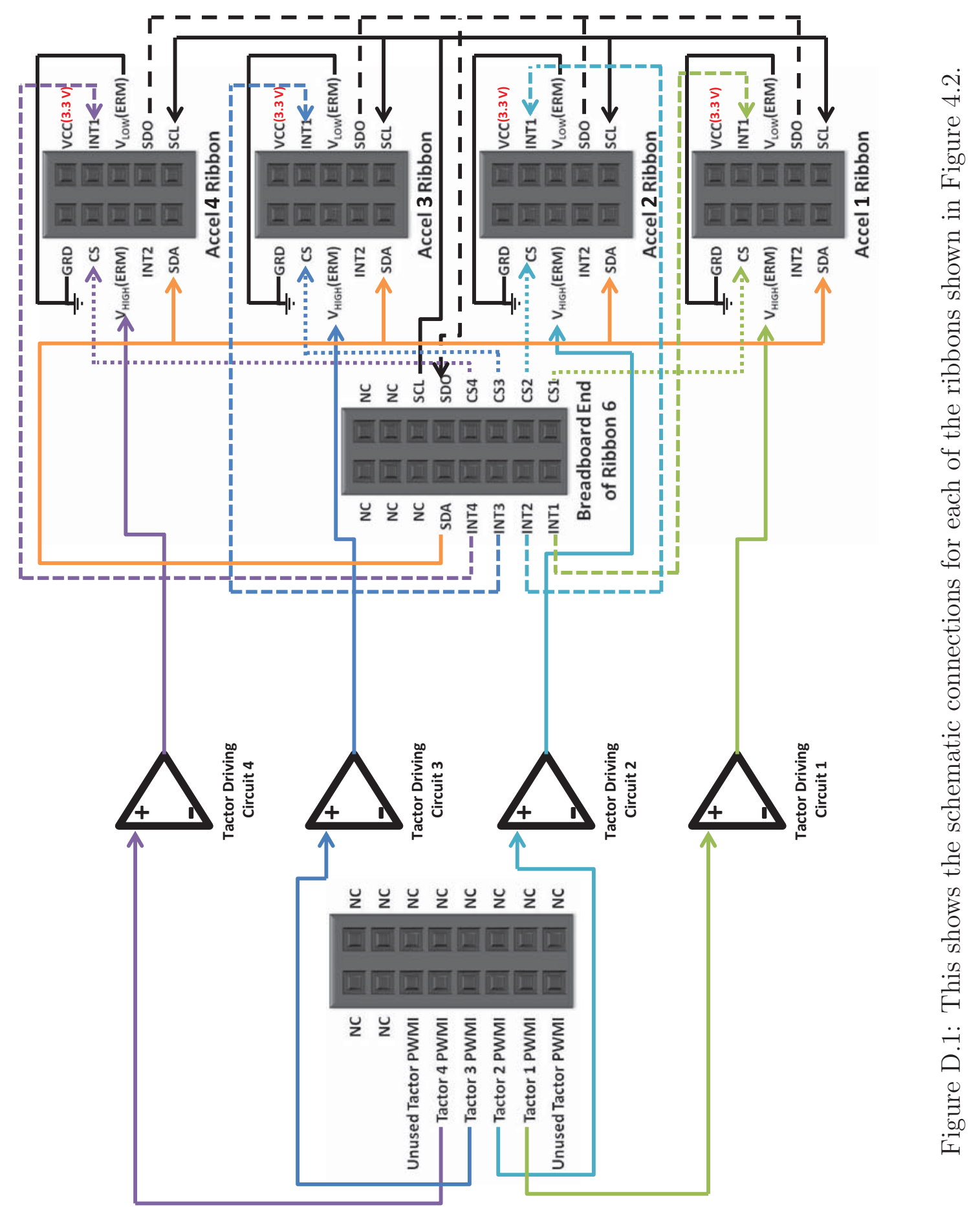




\section{Appendix E}

\section{Arduino Code for Location Optimization}

\section{Testing}

//Add the SPI library so we can communicate with the ADXL345 sensor \#include <SPI.h>

//*****We can have multiple SPI devices connected to the Arduino because when the chip select or slave select (CS) pin

//a device is low $t$ communicates with the master, but when its high, it ignores the master

// The reference website for this code showed the following configuration for connecting to the ADXL345

// Arduino $\Rightarrow$ Accelerometer Board

$/ /$ Pin $10 \Rightarrow C S$

$/ / \operatorname{Pin} 11 \Rightarrow \mathrm{SDA}$

$/ /$ Pin $12 \Rightarrow$ SDO

$/ /$ Pin $13 \Rightarrow$ SCL

$/ / 3 \mathrm{~V} 3 \Rightarrow \mathrm{VCC}$

$/ /$ GND $=>$ GND

// on the Arduino Mega 2560, the SPI pins are broken out as follows

// Pin $53 \Rightarrow$ CS (chip/slave select)

$/ /$ Pin $51 \Rightarrow$ SDA (serial data input to slave)

$/ /$ Pin $50 \Rightarrow$ SDO (serial data output from slave)

$/ /$ Pin $52 \Rightarrow$ SCL (serial clock)

// $3 \mathrm{~V} 3=>\mathrm{VCC}$

$/ /$ GND $=>$ GND

//Assign the Chip Select signal to the various pins that control the accelerometers on different tactors 
$/ /$ int $\mathrm{CS}=53 ; / /$ This is the

int ChipSelect []$=\{42,44,46,48\}$; //tactor 1's motion can be detected by communicating with the accelerometer

//connected to pin 53, tactor 2's motion is communicated through pin 54, Tactor 3 through pin 55, Tactor 4 through pin 56

//I also need to set the INT1 pin for each of the accelerometers

int Interrupt []$=\{43,45,47,49\}$;

//This is a list of some of the registers available on the ADXL345.

//To learn more about these and the rest of the registers on the ADXL345, read the datasheet!

\#define POWER_CTL 0x2D //Power Control Register

\#define BW_RATE 0x2C

\#define DATA_FORMAT 0x31//Measurement rate

\#define DATAX0 0x32//X-Axis Data 0

\#define DATAX1 0x33//X-Axis Data 1

\#define DATAYO 0x34//Y-Axis Data 0

\#define DATAY1 0x35//Y-Axis Data 1

\#define DATAZ0 0x36//Z-Axis Data 0

\#define DATAZ1 0x37 //Z-Axis Data 1

\#define NumPoints (200)//this is the number of points we want to collect to represent the acceleration profile at each body location

$/ / * * * *$ we want the number of points to be 200 , because with 100 , there are too few points/sinewaves to analyse the signal properly, and when filtered in MATLAB we obtain poorer signal properties

//From the datasheet we know that for SPI communication, CPOL=1 and $\mathrm{CPHA}=1$, there fore, according to the arduino page on

//SPI (https://www.arduino.cc/en/Reference/SPI) the SPI mode used is SPI_MODE3, there is no information about the way

//the data is shifted, but its often most significant bit so we'll pickthe MSBFIRST option (as opposed to LSBFIRST)

//\#define mode SPI_MODE3

//This buffer will hold values read from the ADXL345 registers.

$/ /$ char values[10]; //we only want to read 2 values from the accelerometer for each acceleration direction

//These variables will be used to hold the $\mathrm{x}, \mathrm{y}$ and $\mathrm{z}$ axis accelerometer values.

//int $x, y, z$;

int $\mathrm{x} 0$ [NumPoints];

int $\mathrm{x} 1$ [NumPoints];

int y0[NumPoints];

int y1 [NumPoints];

int $z 0$ [NumPoints];

int $\mathrm{z} 1$ [NumPoints]; 


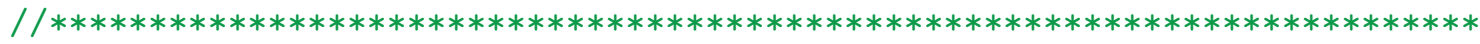

//The following sets the location testing order

byte NumLocations $=5$;

int Location []$=\{3,4,1,5,3\}$;

//For each location being tested (neck, shoulder, arm,...), there is a different order in which tactors are to be activated for each body location (same goes for vibration intensity)

//The following sets the tactor activation order

//THE FOLLOWING IS FOR PARTICIPANTS TO TEST WITH, COMMENT OUT THE TACTOR ORDER FOR THE TESTING LOCATION WHEN TRYING TO GET PARTICPANTS USED TO THE INTENSITIES

//int TactorOrder3[] $=\{1,2,3,4,1,2,3,4,1,2,3,4,1,2,3,4,1,2,3,4,1,2,3,4\}$; //getting used to feedback activation order

int TactorOrder 1[]$=\{4,4,2,3,2,4,2,2,4,3,4,1,3,2,3,1,1,2,3,1,1,1,3,4\}$; //tactor activation order for the neck

int TactorOrder 2[]$=\{2,3,4,1,4,4,4,4,1,3,2,1,1,2,3,3,2,4,1,2,2,3,3,1\}$; //tactor activation order for the head

int TactorOrder 3[]$=\{2,2,4,4,2,3,1,4,2,4,1,2,1,3,3,4,1,1,3,2,3,1,4,3\}$; $/ /$ tactor activation order for the arm/wrist

int TactorOrder 4[]$=\{1,4,1,4,1,4,1,2,4,4,3,3,2,3,1,2,2,3,2,1,2,3,3,4\}$; $/ /$ tactor activation order for the waist

int TactorOrder5[] $=\{4,3,4,3,1,3,4,2,4,2,1,3,2,2,3,4,1,3,4,1,2,1,2,1\}$; //tactor activation order for the ankle

//The following sets the tactor activation intensity order **THESE VALUES NEED TO CHANGE FOR EACH PARTICIPANT

\#define L 40 //Low Intensity -- the intensity is for the PWM signal (out of 255), the TYPICAL start-up voltage for the ERM is $0.8 \mathrm{~V}$, if we choose $\mathrm{L}=40$, the voltage across the tactor would be $40 / 255 * 5 \mathrm{~V}=0.78 \mathrm{~V}$, a safe amount above the MINIMUM start-up voltage (0.35V)

\#define H 87 //High Intensity $\Rightarrow$ max voltage that can be inputed to the MAX4019 is $1.7 \mathrm{~V}$, afterwhich, there is no real difference in voltage intensity, therefore $\mathrm{H}=1.7 \mathrm{~V} / 5 \mathrm{~V} * 255=87$ (this output 1.7 volts, just shy of the max voltage for the ERM)

//THE FOLLOWING IS THE HIGH VOLTAGE FOR THE HEAD, BECAUSE WE DON'T WANT THE HIGH VOLTAGE TO BE ANYWHERE NEAR WHAT IT IS FOR OTHER PARTS OF THE BODY

\#define h 87

\#define 140 //was 36

/*int TactorIntensity1 []$=\{L, L, L, L, L, L, L, L, L, L, L, L, L, L, L, L\} ; / /$ tactor activation intensity for the neck

int TactorIntensity 2[]$=\{\mathrm{L}, \mathrm{L}, \mathrm{L}, \mathrm{L}, \mathrm{L}, \mathrm{L}, \mathrm{L}, \mathrm{L}, \mathrm{L}, \mathrm{L}, \mathrm{L}, \mathrm{L}, \mathrm{L}, \mathrm{L}, \mathrm{L}, \mathrm{L}\}$; //tactor activation intensity for the shoulder

int TactorIntensity 3[]$=\{\mathrm{L}, \mathrm{L}, \mathrm{L}, \mathrm{L}, \mathrm{L}, \mathrm{L}, \mathrm{L}, \mathrm{L}, \mathrm{L}, \mathrm{L}, \mathrm{L}, \mathrm{L}, \mathrm{L}, \mathrm{L}, \mathrm{L}, \mathrm{L}\}$; //tactor activation intensity for the arm/wrist 
int TactorIntensity 4[]$=\{\mathrm{L}, \mathrm{L}, \mathrm{L}, \mathrm{L}, \mathrm{L}, \mathrm{L}, \mathrm{L}, \mathrm{L}, \mathrm{L}, \mathrm{L}, \mathrm{L}, \mathrm{L}, \mathrm{L}, \mathrm{L}, \mathrm{L}, \mathrm{L}\} ; / /$ tactor activation intensity for the waist

int TactorIntensity5[]=\{L, L, L, L, L, L, L, L, L, L, L , L , L , L , L , L $\}$; //tactor activation intensity for the ankle

$* /$

//The following are the actual intensities, we only used the above to test the ability to read the acceleration

//int

TactorIntensity3 [] = $\{\mathrm{L}, \mathrm{L}, \mathrm{L}, \mathrm{L}, \mathrm{H}, \mathrm{H}, \mathrm{H}, \mathrm{H}, \mathrm{L}, \mathrm{L}, \mathrm{L}, \mathrm{L}, \mathrm{H}, \mathrm{H}, \mathrm{H}, \mathrm{H}, \mathrm{L}, \mathrm{L}, \mathrm{L}, \mathrm{L}, \mathrm{H}, \mathrm{H}, \mathrm{H}, \mathrm{H}\}$;

//getting used to feedback activation intensity

int TactorIntensity 1[]$=\{H, L, L, L, H, L, H, L, L, H, H, L, H, H, L, H, L, L, L, H, H, L, H, H\}$;

//tactor activation intensity for the neck

int TactorIntensity 2[]$=\{H, L, H, H, L, H, L, H, L, H, H, H, L, L, H, L, L, L, L, H, L, L, H, H\}$; //tactor activation intensity for the head

int TactorIntensity []$=\{\mathrm{H}, \mathrm{H}, \mathrm{H}, \mathrm{L}, \mathrm{H}, \mathrm{L}, \mathrm{H}, \mathrm{L}, \mathrm{L}, \mathrm{H}, \mathrm{L}, \mathrm{L}, \mathrm{H}, \mathrm{H}, \mathrm{L}, \mathrm{L}, \mathrm{L}, \mathrm{H}, \mathrm{H}, \mathrm{L}, \mathrm{H}, \mathrm{L}, \mathrm{H}, \mathrm{L}\}$; //tactor activation intensity for the arm/wrist

int TactorIntensity 4[]$=\{L, L, H, H, L, H, H, L, H, L, L, L, H, H, H, H, L, H, H, L, L, L, H, L\}$; //tactor activation intensity for the waist

int TactorIntensity5[] $=\{\mathrm{L}, \mathrm{L}, \mathrm{H}, \mathrm{L}, \mathrm{H}, \mathrm{H}, \mathrm{H}, \mathrm{H}, \mathrm{L}, \mathrm{H}, \mathrm{H}, \mathrm{H}, \mathrm{L}, \mathrm{L}, \mathrm{L}, \mathrm{L}, \mathrm{L}, \mathrm{H}, \mathrm{H}, \mathrm{H}, \mathrm{L}, \mathrm{L}, \mathrm{H}, \mathrm{L}\}$; //tactor activation intensity for the ankle

//The following sets the time delay between tactor activations to prevent users from pressing on the button not because they feel the feedback, but because they actually know that once one tactor stops vibrating, another starts to vibrate

//the delays are in milliseconds

//int

ActivationDelay3 []$=\{1500,1500,1000,1500,500,1500,2500,500,1000,2000, \ldots$

$\ldots 1000,2500,2500,2000,1500,2000,2500,2000,500,1000,500,500,2000,1000\}$;

//getting used to tactor activation intensity

int ActivationDelay1 []$=\{2500,2000,1500,1000,1500,2000,1000,500,2000,500, \ldots$ ...2500, 2000, 500, 500, 1500, 1000, 2000, 2500, 2500, 1000, 1500, 1500, 1000, 500\};

//tactor activation intensity for the neck int ActivationDelay2 []$=\{2000,1000,2500,500,1000,500,1500,2000,2000,2000, \ldots$ $\ldots 1000,2500,2000,1000,1500,1500,2500,1000,2500,500,500,1500,500,1500\}$;

//tactor activation intensity for the shoulder int ActivationDelay3 []$=\{1000,2500,2500,500,1500,1500,1000,500,1500,2000, \ldots$ ...2000,1000,2000,2500,1500,2000,1000,1000,500, 2500, 500,1500, 2000, 500\};

//tactor activation intensity for the arm/wrist int ActivationDelay4 []$=\{2000,1000,500,500,2500,1500,2500,2000,2500,1500, \ldots$ ...2000,2000,500,500,2000,1000,1500,1000,1500,1000,500,1500, 2500, 1000\};

$/ /$ tactor activation intensity for the waist int ActivationDelay5 []$=\{1000,1500,2500,2000,500,2500,2500,1500,2000,500, \ldots$ ...2500,1000,1000,1500,500,2000,1500,500, 2000,1000, 2000,1500, 500, 1000\};

//tactor activation intensity for the ankle 
byte ArrayLength=24; //this is the length of the above arrays for tactor and activation intensity

//The following states which Arduino pins control which tactor byte TactorPin []$=\{9,10,11,12\}$;

//define the pins that power the un-used op-amps on the MAX4019 byte UnconnectedOpAmp1=13;

byte UnconnectedOpAmp2=8;

//The following states which pins are used to read the outputs generated by buttons pressed

int Button []$=\{22,24,26,28,30,32,34,36\}$;

$\operatorname{void} \operatorname{setup}()\{$

//Create a serial connection to display the data on the terminal.

Serial.begin(9600);

//The following sets the pins on the arduino as input or output based on the 4-wire SPI connection shown on the datasheet

// (https://www.sparkfun.com/datasheets/Sensors/Accelerometer/ADXL345.pdf) pinMode(51, OUTPUT); //set the SDA pin as output from arduino

pinMode(50, INPUT); //set the SDO pin as input to arduino

pinMode(52, OUTPUT); //set the SCL pin as output

//Set the pins for Chip Select and Interrupt to Ouput

for (byte $i=0 ; i<=3 ; i++)\{$

pinMode (Interrupt [i], OUTPUT);

pinMode(ChipSelect [i], OUTPUT);

pinMode (TactorPin [i], OUTPUT);

\}

//set the pins controlling the unconnected opamps as outputs pinMode (UnconnectedOpAmp1, OUTPUT);

pinMode (UnconnectedOpAmp2, OUTPUT);

//set the pins for the buttons used to indicate if the participant felt the feedback

for (byte $i=0 ; i<=7 ; i++)\{$

pinMode (Button [i], INPUT);

\}

//set the pin for the button stopping the code from moving on until the tactors are attached to the next location to INPUT

pinMode (31, INPUT) ; 


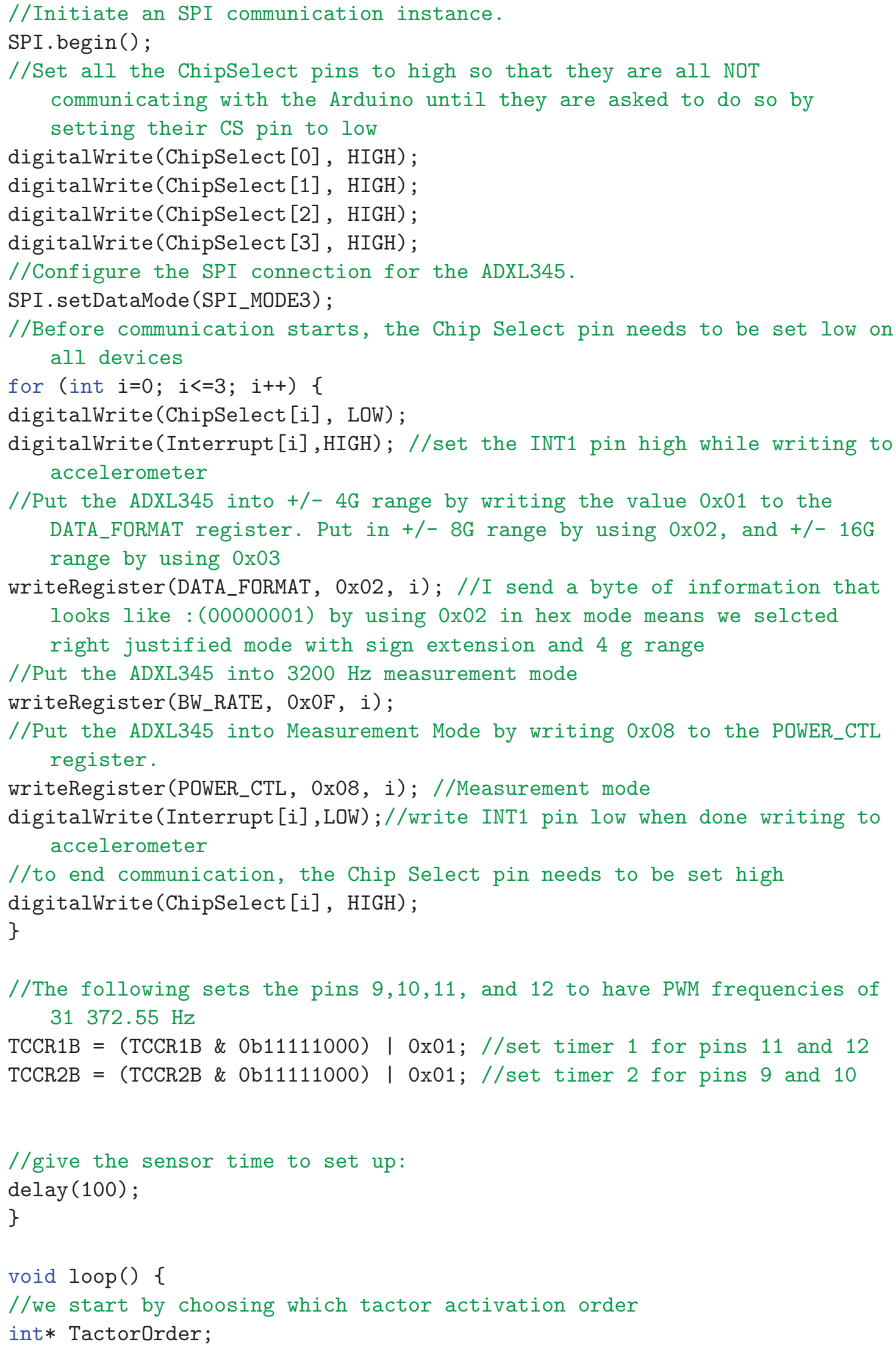




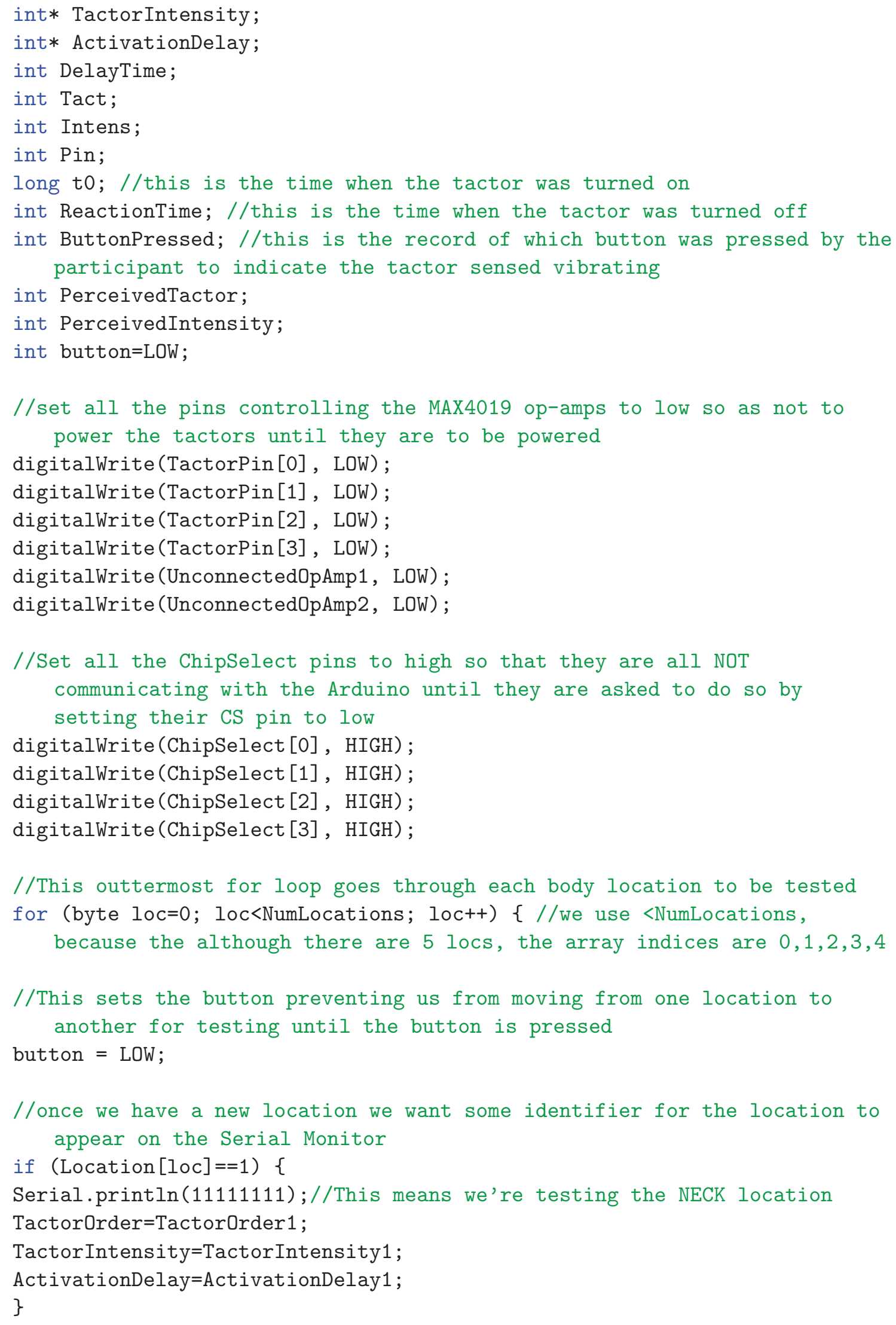




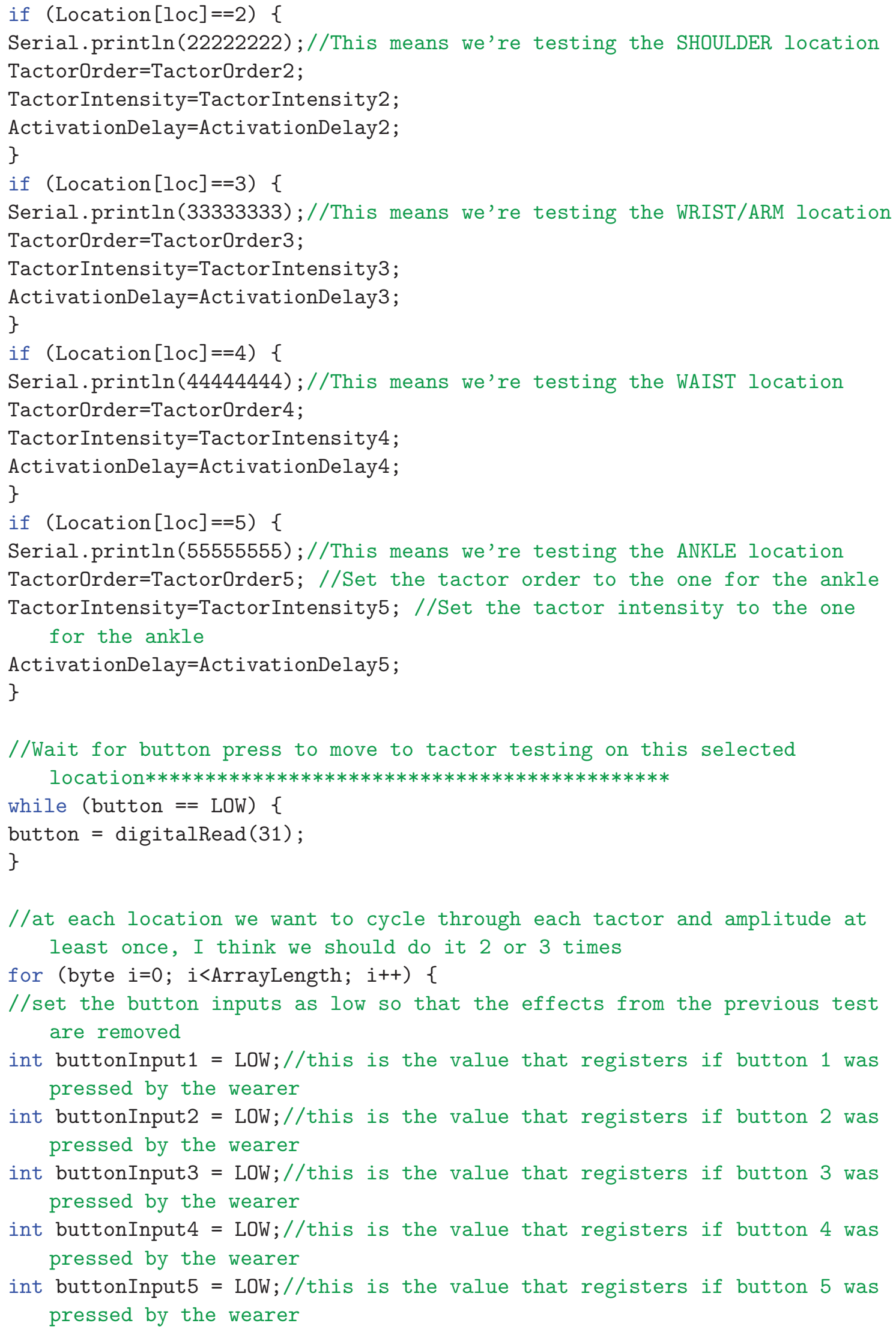


int buttonInput6 $=\mathrm{LOW}$;//his is the value that registers if button 6 was pressed by the wearer

int buttonInput7 $=\mathrm{LOW} ; / /$ this is the value that registers if button 7 was pressed by the wearer

int buttonInput8 = LOW;//this is the value that registers if button 8 was pressed by the wearer

//set the ButtonPressed as zero so that if the maximum time elapses

without the participant pressing a button, we won't accidentally

report that they pressed a button

int ButtonPressed=0;

//we want to start by activating the tactor according to the order above Tact=TactorOrder[i];//these values vary from 1,2,3 or 4

Intens=TactorIntensity[i];//these values are either L (low) or H (high)

Pin=TactorPin[Tact-1];//there are 4 elements in the TactorPin array, so we need to have the index ranging from 0 to 3 , hence Tact-1 is used as the index

DelayTime=ActivationDelay [i];

delay(DelayTime);//random delay in ms length before each tactor activation

analogWrite(Pin,Intens);//turn on the tactor at the set intensity

//get the start time so can find the reaction time $\Rightarrow>$ used millis, not

micros because typically the average reaction time is in the range of 100 miliseconds

t0 $=$ millis () ;

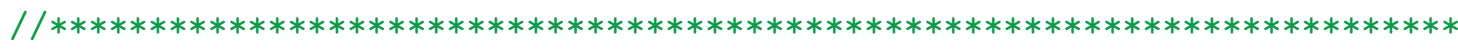

//check if one of the buttons is pressed (maybe make a while loop saying all the pins are low )

long $j=0 ; / /$ this is the counter used to make sure that the code exits after a certain elapsed time

while (buttonInput1 == LOW \&\& buttonInput2 $==$ LOW \&\& buttonInput3 == LOW

$\& \&$ buttonInput $4==$ LOW $\& \&$ buttonInput5 $==$ LOW \&\& buttonInput6 $==$ LOW

\&\& buttonInput7 $==$ LOW \&\& buttonInput8 $==$ LOW \&\& $j<120000) \quad\{$

buttonInput8 = digitalRead (22);

buttonInput7 = digitalRead (24);

buttonInput6 = digitalRead (26);

buttonInput5 = digitalRead (28);

buttonInput4 = digitalRead (30);

buttonInput3 = digitalRead (32);

buttonInput2 = digitalRead (34);

buttonInput 1 = digitalRead (36);

$j=j+1$;

\} 


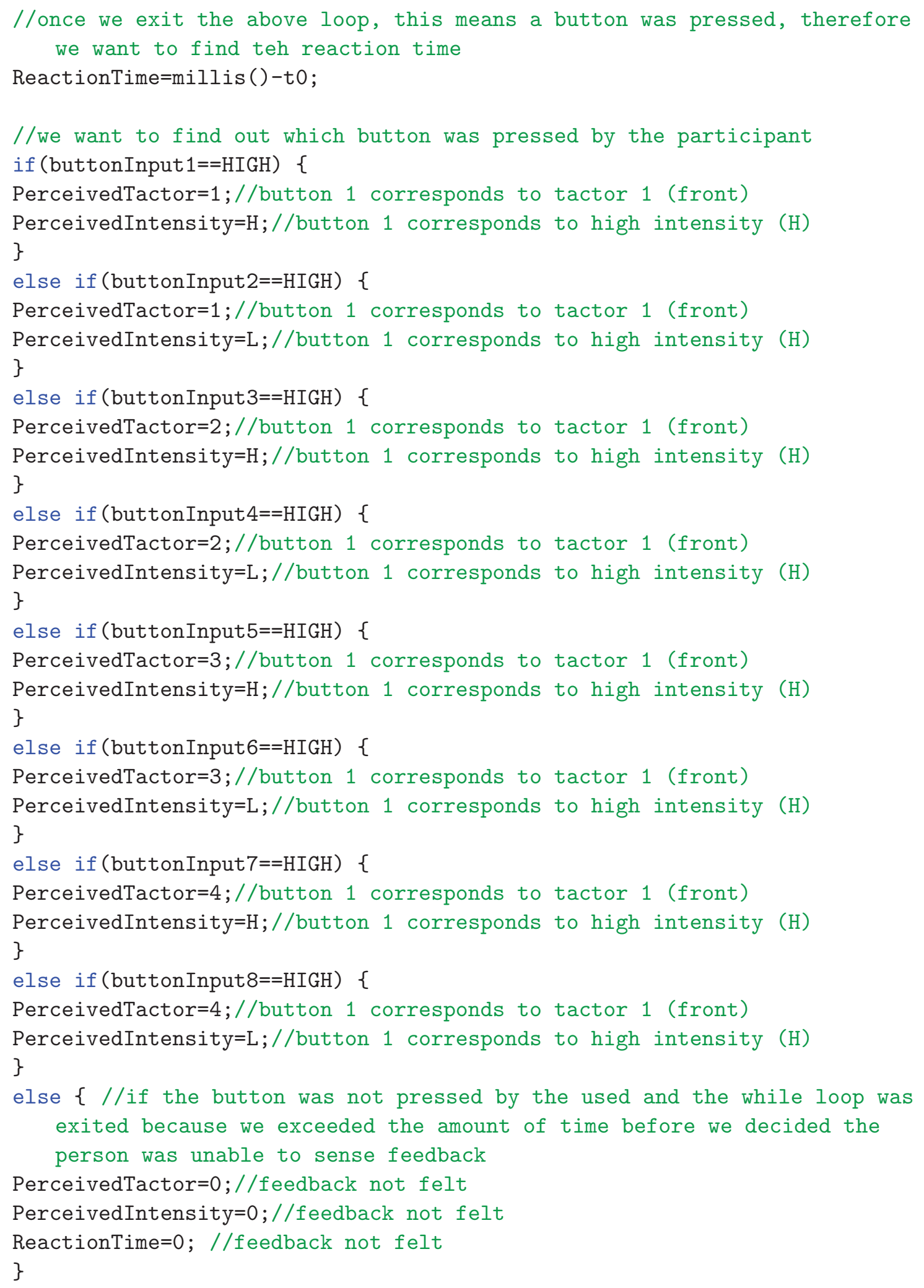


//print off the information regarding the tactor activated and the actual vibration intensity, the participant's identified

$/ /$ tactor activated, the tactor intensity detected, the reaction time, the acceleration data

Serial.println(88888888);//this marks the start of a new tactor activation sequence (the information that comes in the next row is as follows)

Serial.print(Tact, DEC);//actual tactor activated

Serial.print(', ');

Serial.print(Intens, DEC);//actual vibration intensity

Serial.print(', ');

Serial.print(PerceivedTactor, DEC);//perceived tactor activated

Serial.print(',');

Serial.print(PerceivedIntensity, DEC);//perceived tactor vibration intensity

Serial.print (', ');

Serial.print(ReactionTime, DEC);//reaction time

Serial.println();

readRegister(Tact-1);//we want to target the element in chip select that corresponds to the correct accelerometer on the correct tactor based on the tacotr activated (Tact)

digitalWrite(Pin,LOW);//turn off the tactor

delay(10); //the delay between tests should be random to prevent users from \}

\}

\}

//This function will write a value to a register on the ADXL345. //Parameters:

// char registerAddress - The register to write a value to

// char value - The value to be written to the specified register.

void writeRegister(char registerAddress, char value, int i) \{

//Set Chip Select pin low to signal the beginning of an SPI packet. digitalWrite(ChipSelect [i], LOW);

//Set the INT1 pin high when writing to accelerometer digitalWrite (Interrupt [i], HIGH);

//Transfer the register address over SPI.

SPI.transfer (registerAddress);

//Transfer the desired register value over SPI.

SPI.transfer (value);

//set INT1 pin low when done writing to accelerometer digitalWrite(Interrupt [i], LOW);

//Set the Chip Select pin high to signal the end of an SPI packet.

digitalWrite(ChipSelect [i], HIGH); 
//This function will read a certain number of registers starting from a specified address and store their values into the appropriate byte for the corresponding acceleration direction

void readRegister (int i) \{

//I WILL TRY TO GIVE EACH OF THE FOLLOWIGN BYTE A VALUE AS IT SHOULD BE

GIVEN BY THE ADXL AND THEN PRINT IT TO THE SERIAL MONITOR

/*unsigned char $\mathrm{x} 0=0$;

unsigned char $\mathrm{x} 1=0$;

unsigned char $\mathrm{y} 0=0$;

unsigned char $\mathrm{y} 1=0$;

unsigned char $\mathrm{z} 0=0$;

unsigned char $\mathrm{z} 1=0$;

$* /$

//begin communicating with the accelerometer

SPI.beginTransaction (SPISettings (5000000, MSBFIRST, SPI_MODE3));//from

datasheet, ADXL345 max SPI clock frequency is $5 \mathrm{MHz}, \mathrm{CPOL}=1$ and

$\mathrm{CPHA}=1$, therefore, according to the arduino page on SPI

(https://www.arduino.cc/en/Reference/SPI) the SPI mode used is SPI_MODE

for (int $j=0 ; j<$ NumPoints; $j++$ ) \{

//set the chip select pin low to begin transaction with accelerometer

digitalWrite(ChipSelect [i] ,LOW);

//set INT1 pin high to write to the device

digitalWrite(Interrupt [i], HIGH);

//write the address for the $\mathrm{x}$ acceleration's LSbyte, therefore the first

byte received will correspond to $\mathrm{x}$ 's LSbyte and the second to $\mathrm{x}$ 's

MSbyte, and so on so forth (as mentioned in the application sheet and datasheet)

SPI.transfer ( (0x32|0x80)|0x40);

//stop writing to the chip

digitalWrite(Interrupt [i], LOW);

//send a value of zero to read the first byte returned, which should

correspond to the $\mathrm{x} 0$ byte (or least significant byte corresponding to the $\mathrm{x}$ acceleration), and then to every other byte of interest

$\mathrm{x} 0[\mathrm{j}]=$ SPI.transfer $(0 \mathrm{x} 00) ;$

$\mathrm{x} 1[\mathrm{j}]=$ SPI.transfer $(0 \mathrm{x} 00) ;$

$\mathrm{y} 0[j]=$ SPI.transfer $(0 \mathrm{x} 00)$;

$\mathrm{y} 1[j]=$ SPI.transfer $(0 \mathrm{x} 00)$;

$z 0[j]=$ SPI.transfer $(0 \times 00) ;$

$z 1[j]=$ SPI.transfer $(0 \times 00) ;$

//Set the Chip select pin high to end an SPI packet.

digitalWrite(ChipSelect [i], HIGH); 
delayMicroseconds(500); //because the SPI communication is so fast, if we don't insert a delay we barely get enough data to represent 1 period when using 200 points, by entering this delay we get about 9-10 vibration cycles using 200 points at a low vibration voltage, 21 \} points are used to represent each period

//try to convert the two bytes for each direction into one value to be printed; THIS CODE IS COMMENTED OUT BECAUSE IT WASN'T WORKING, THE APPLICATION SHEET FOR

//THE ADXL345 MENTIONS THAT THE ACCELERATION INFORMATION IS ENCODED IN TWOS COMPLEMENT FORMAT, BUT I'M NOT SURE THE ARDUINO PROGRAM IS ABLE TO PARSE IT PROPERLY

//GIVEN THE FAULTY RESULTS OBSERVED WHEN CONSIDERING THE INFORMATION //unsigned int $\mathrm{x}=\mathrm{x} 1<8$ | $\mathrm{x} 0$;

//unsigned int $\mathrm{y}=($ (unsigned int) $\mathrm{y} 1<<8)+$ (unsigned int) $\mathrm{y} 0$;

//unsigned int $z=(($ unsigned int) $z 1<8)+$ (unsigned int) $z 0$;

//end all communication with the ADXL345

SPI.endTransaction();

//try printing acceleration information as binary values so I can edit the data in MATLAB; remember that the data for $\mathrm{x} 1$ is the MSbyte (therefore should be on the left) and the data for $\mathrm{x} 0$ is the LSbyte

$/ /$ (therefore should be on the right) $\Rightarrow$ note however that MATBLAB seems to hold the LSB as the leftmost value in an array

for (int $j=0 ; j<$ NumPoints; $j++$ ) \{

Serial.print ( $\mathrm{x} 0[\mathrm{j}], \mathrm{DEC})$;

Serial.print (', ');

Serial.print (x1[j], DEC);

Serial.print (',');

Serial.print (y0 $[j], \mathrm{DEC})$;

Serial.print(', ');

Serial.print (y1 $[j], \mathrm{DEC})$;

Serial.print(', ');

Serial.print $(z 0[j], D E C)$;

Serial.print(',');

Serial.print (z1[j], DEC);

Serial.println();

\}

\} 


\section{Appendix F}

\section{Linear Regression of Evaluation Criteria to BMI Results}

The following table contains the correlation coefficient values as well as the p values used to demonstrate the significance of the linear relationship between both the evaluation criteria values and their corresponding BMI values obtained by running individual linear regressions on the data for each combination of evaluation criteria and body location. Should the p values be smaller than 0.05, this would identify a significant linear relationship between the evaluation criteria and the BMI. No p value is below 0.05 , therefore it can be said that no linear relationship exists between any of the evaluation criteria and BMI.

Table F.1: Correlation coefficients ( $\mathrm{p}$ values) obtained from the linear regression analysis between each evaluation criterion and BMI

\begin{tabular}{lllll}
\hline Evaluation & \multicolumn{4}{c}{ Body Location } \\
Criteria & Neck & Wrist & Waist & Ankle \\
\hline Reaction Time & $.015(.967)$ & $.136(.708)$ & $.125(.731)$ & $.316(.373)$ \\
\%Detected & NA & $.072(.844)$ & $.392(.262)$ & $.036(.920)$ \\
\% Tactor & $.289(.418)$ & $.261(.466)$ & $.311(.381)$ & $.192(.596)$ \\
\%Intensity & $.485(.155)$ & $.410(.239)$ & $.114(.754)$ & $.158(.663)$ \\
\hline
\end{tabular}




\section{Appendix G}

\section{Linear Regression of Acceleration and Displacement Amplitude to BMI Results}

Table G.1: Parameters related to the linear regression between various acceleration amplitude data and the corresponding participant BMI

\begin{tabular}{ccccccccc}
\hline \multirow{2}{*}{$\begin{array}{c}\text { Body } \\
\text { Location }\end{array}$} & $\begin{array}{c}\text { Accel. } \\
\text { Amp. }\end{array}$ & \multicolumn{3}{c}{ Model Summary } & ANOVA & \multicolumn{3}{c}{ Regression Equation Information } \\
& Direction & $R$ & $R^{2}$ & p value & Value & Sig. & Value & Sig. \\
\hline \multirow{3}{*}{ Neck } & $\mathrm{X}$ & .043 & .002 & .906 & 55.116 & .482 & .369 & .906 \\
& $\mathrm{Y}$ & .002 & .000 & .995 & 12.469 & .580 & -.006 & .995 \\
& $\mathrm{Z}$ & .413 & .170 & .236 & 10.708 & .014 & -.176 & .236 \\
& $\mathrm{X}$ & .233 & .054 & .517 & 110.951 & .213 & -2.247 & .517 \\
Wrist & $\mathrm{Y}$ & .183 & .033 & .613 & 12.102 & .014 & -.082 & .613 \\
& $\mathrm{Z}$ & .606 & .368 & .063 & -3.282 & .333 & .278 & .063 \\
& $\mathrm{X}$ & .427 & .182 & .218 & -42.488 & .559 & 3.760 & .218 \\
Waist & $\mathrm{Y}$ & .455 & .207 & .186 & 36.332 & .044 & -.886 & .186 \\
& $\mathrm{Z}$ & .522 & .272 & .122 & 2.602 & .270 & .153 & .122 \\
& $\mathrm{X}$ & .183 & .034 & .612 & 68.469 & .288 & -1.282 & .612 \\
Ankle & $\mathrm{Y}$ & .106 & .011 & .771 & 12.853 & .032 & .060 & .771 \\
& $\mathrm{Z}$ & .102 & .010 & .779 & 1.997 & .333 & .023 & .779 \\
\hline
\end{tabular}


Table G.2: Parameters related to the linear regression between various displacement amplitude data and the corresponding participant BMI

\begin{tabular}{ccccccccc}
\hline \multirow{2}{*}{$\begin{array}{c}\text { Body } \\
\text { Location }\end{array}$} & Disp. & \multicolumn{2}{c}{ Model Summary } & ANOVA & \multicolumn{3}{c}{ Regression Equation Information } \\
& Amp. & $R$ & $R^{2}$ & $\mathrm{p}$ value & \multicolumn{2}{c}{ Intercept } & \multicolumn{2}{c}{ Slope } \\
& Direction & & & & Value & Sig. & Value & Sig. \\
\hline \multirow{3}{*}{ Neck } & $\mathrm{X}$ & .138 & .019 & .704 & .135 & .947 & .031 & .704 \\
& $\mathrm{Y}$ & .502 & .252 & .139 & 1.144 & .070 & -.036 & .139 \\
& $\mathrm{Z}$ & .720 & .518 & .019 & .779 & .007 & -.026 & .019 \\
& $\mathrm{X}$ & .498 & .248 & .143 & -.893 & .272 & .050 & .143 \\
Wrist & $\mathrm{Y}$ & .510 & .260 & .132 & -.079 & .327 & .005 & .132 \\
& $\mathrm{Z}$ & .416 & .173 & .232 & -.114 & .339 & .006 & .232 \\
& $\mathrm{X}$ & .341 & .116 & .335 & -5.082 & .463 & .273 & .335 \\
Waist & $\mathrm{Y}$ & .061 & .004 & .866 & .052 & .815 & .002 & .866 \\
& $\mathrm{Z}$ & .228 & .052 & .527 & -.132 & .709 & .009 & .527 \\
\multirow{5}{*}{ Ankle } & $\mathrm{X}$ & .076 & .006 & .835 & .092 & .982 & .034 & .835 \\
& $\mathrm{Y}$ & .208 & .043 & .564 & .067 & .045 & -.001 & .564 \\
& $\mathrm{Z}$ & .021 & .000 & .953 & .021 & .552 & .000 & .953 \\
\hline
\end{tabular}




\section{Appendix $\mathbf{H}$}

\section{Arduino Code for Balance Effects Testing}

//THIS CODE WORKS BUT ONLY ALLOWS US TO RUN THROUGH ONE TEST FOR ONE TRIAL AT A TIME, //THE ONLY DIFFERENCE BETWEEN THIS VERSION AND v6 IS THAT THE TILT LIMITS HAVE BEEN CHANGED FROM 3 AND

//5 DEGREES TO 1 AND 3 DEGREES

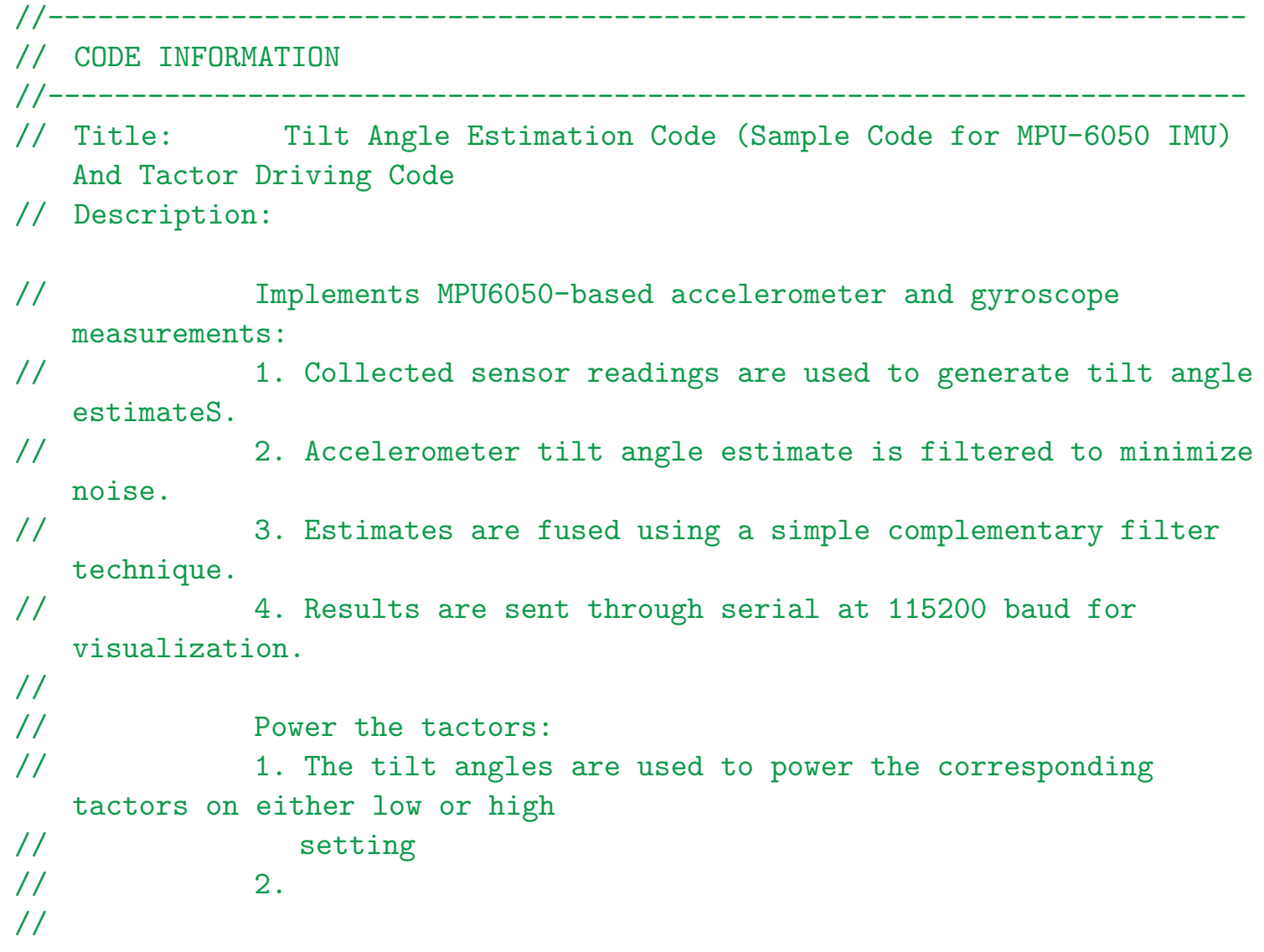


// Please note, there is another way to do the tilt-angle estimation, which you

// can explore if you are curious:

// 1. Simply use the gyroscope measurements as I did here

// 2. Low-pass filter the output to remove integration error

$/ /$

// Author: Omar Masaud @ CU ABL

// Last Edited: 26/09/2016

// Revision History: 26/09/2016 - Initial Release Candidate.

//MEANING OF CODES TO WRITE ON NOTEPAD FILE

// 11110000 -- Trial 1 (eyes open on hard surface) with no feedback

// 11111111 -- Trial 1 (eyes open on hard surface) with feedback on ankle

// 11112222 -- Trial 1 (eyes open on hard surface) with feedback on waist

// 22220000 -- Trial 2 (eyes closed on hard surface) with no feedback

// 22221111 -- Trial 2 (eyes closed on hard surface) with feedback on ankle

// 2222222 -- Trial 2 (eyes closed on hard surface) with feedback on waist

// 33330000 -- Trial 3 (eyes open on foam-covered surface) with no feedback

// 33331111 -- Trial 3 (eyes open on foam-covered surface) with feedback on ankle

// 33332222 -- Trial 3 (eyes open on foam-covered surface) with feedback on waist

// 44440000 -- Trial 4 (eyes closed on foam-covered surface) with no feedback

// 44441111 -- Trial 4 (eyes closed on foam-covered surface) with feedback on ankle

// 44442222 -- Trial 4 (eyes closed on foam-covered surface) with feedback on waist

//For each of the tests, we need to plug in the correct feedback option (either connect the ankle

$/ /$ tactors, the waist tactors or no tactors depending on the type of feedback that corresponds to the

//code that appears on the serial port)

//MEANING OF CODES APPEARING ON SERIAL PORT

//

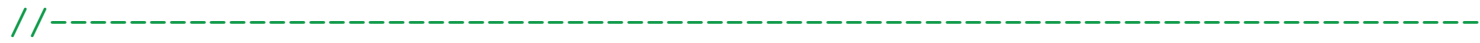

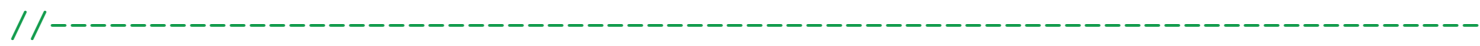

// DEPENDENCIES

\#include "Wire.h" // To communicate with the MPU6050 board using I2C

$/ /-$

//WE NEED TO CHANGE THE FOLLOWING VALUES FOR EACH NEW PARTICIPANT:

//int Trial []$=\{1,2,3,4\}$; 
//int FeedbackCondition []$=\{0,1,2\}$;

$/ /$

//WE NEED TO CHANGE THE FOLLOWING VALUE FOR EVERY TEST:

\#define NumFeedbackCondition 0 //ranges from 0 to 2 for every trial -- the test we are on for the given

//trial (0 if its the first test for this participant, 1 for the second test, 2 for the third test)

//WE NEED TO CHANGE THE FOLLOWING VALUE FOR EVERY TRIAL (EVERY 3 TESTS): \#define NumTrial $0 / /$ ranges from 0 to 3 --this is which trial we are on (0 if its the first trial for

//this participant, 1 for the second trial, 2 for the third trial and 3 for the fourth trial)

\#define TimeDelay $0 / /$ delay between off and on vibrations

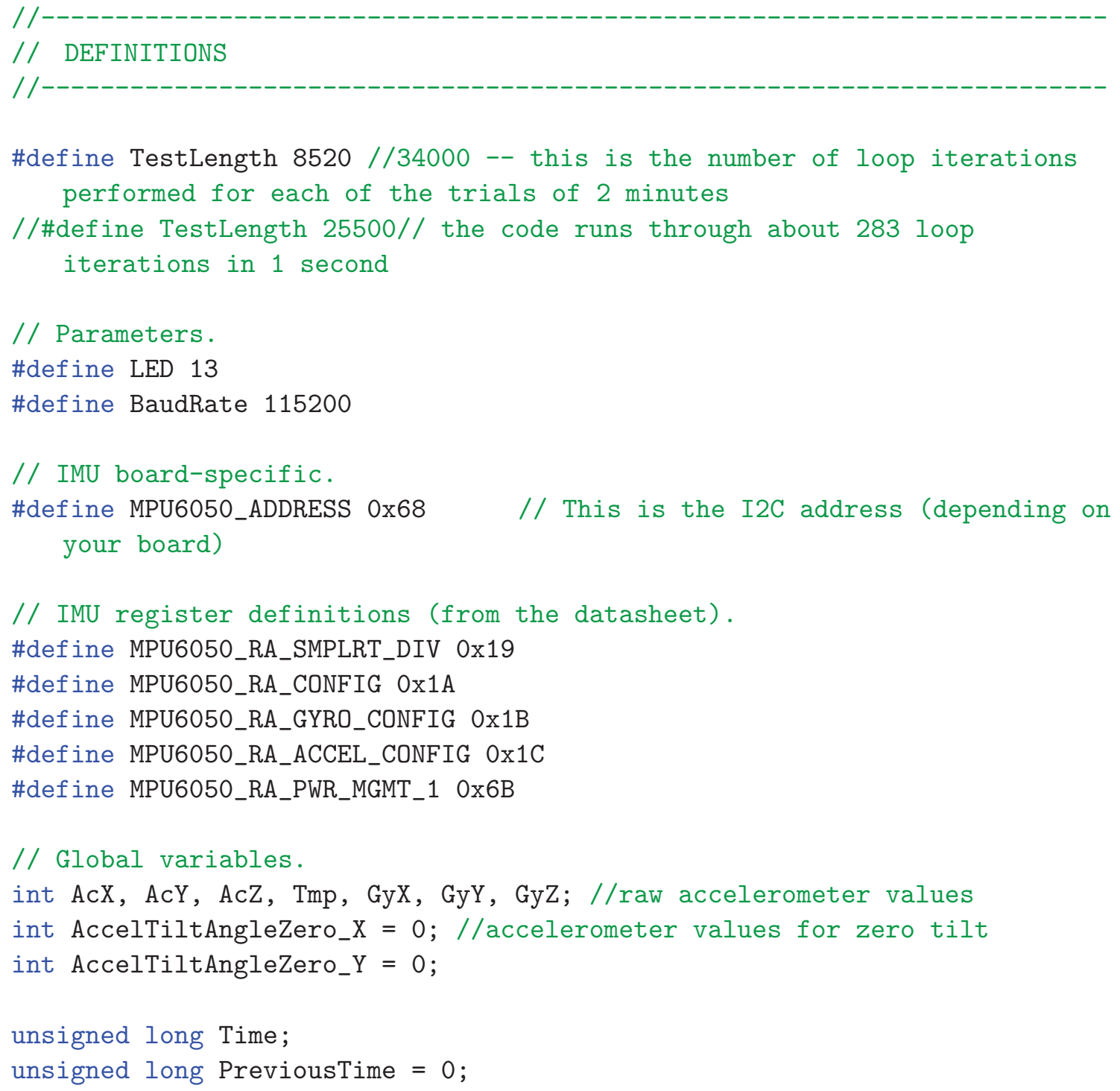




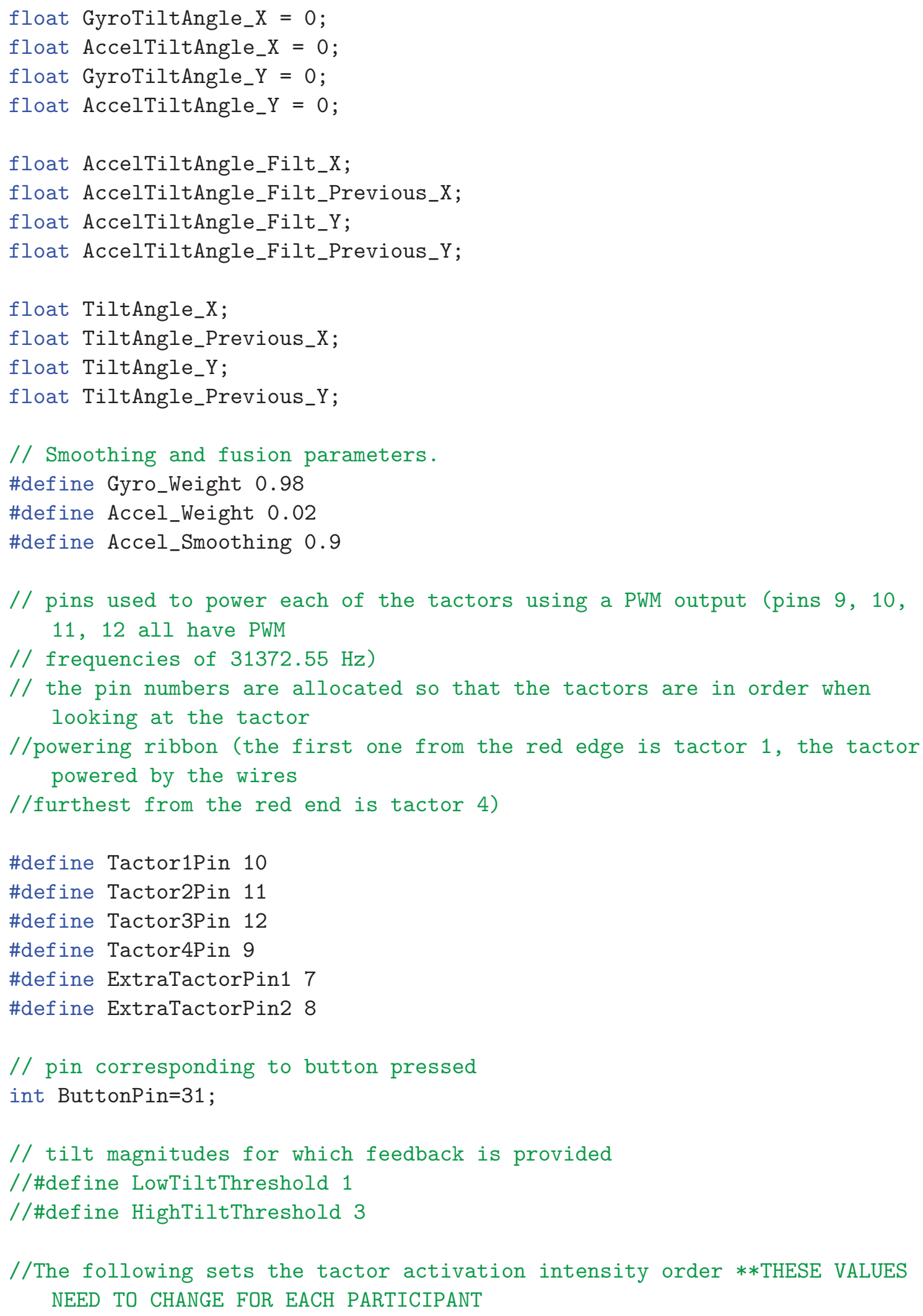


\#define L 46 //Low Intensity -- the intensity is for the PWM signal (out of 255), the TYPICAL start-up

//voltage for the ERM is $0.9 \mathrm{~V}$, if we choose $\mathrm{L}=46$, the voltage across the tactor would be $46 / 255 * 5 \mathrm{~V}=0.90 \mathrm{~V}$,

//a safe amount above the MINIMUM start-up voltage (0.35V)

\#define H 87 //High Intensity => max voltage that can be inputed to the MAX4019 is $1.7 \mathrm{~V}$, afterwhich,

$/ /$ there is no real difference in voltage intensity, therefore $\mathrm{H}=1.7 \mathrm{~V} / 5 \mathrm{~V} * 255=87$ (this output 1.7 volts,

//just shy of the max voltage for the ERM)

//

$/ /$

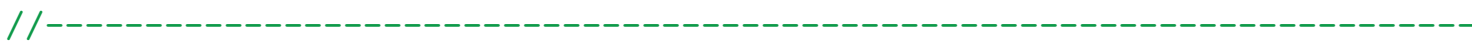

// INITIALIZATION

void $\operatorname{setup}()$

\{

// This function initializes Arduino peripherals.

// Initialize I2C peripheral for communication with MPU6050 IMU.

Wire.begin();

Wire.setClock(100000L);

// LED for debugging.

pinMode (LED, OUTPUT);

digitalWrite (LED, LOW);

// Initialize UART peripheral for communication with PC.

Serial.begin(BaudRate);

// Set up the IMU chip.

Setup_MPU6050();

Calibrate_MPU6050();

// We have communication, so turn the LED high!

digitalWrite (LED, HIGH);

// Set the pins controlling the tactors using PWM as outputs

pinMode (Tactor1Pin, OUTPUT);

pinMode (Tactor2Pin, OUTPUT);

pinMode (Tactor3Pin, OUTPUT);

pinMode (Tactor4Pin, OUTPUT);

pinMode(ExtraTactorPin1, OUTPUT);

pinMode (ExtraTactorPin2, OUTPUT);

// Set the pin that reads whether or not the button was pressed 


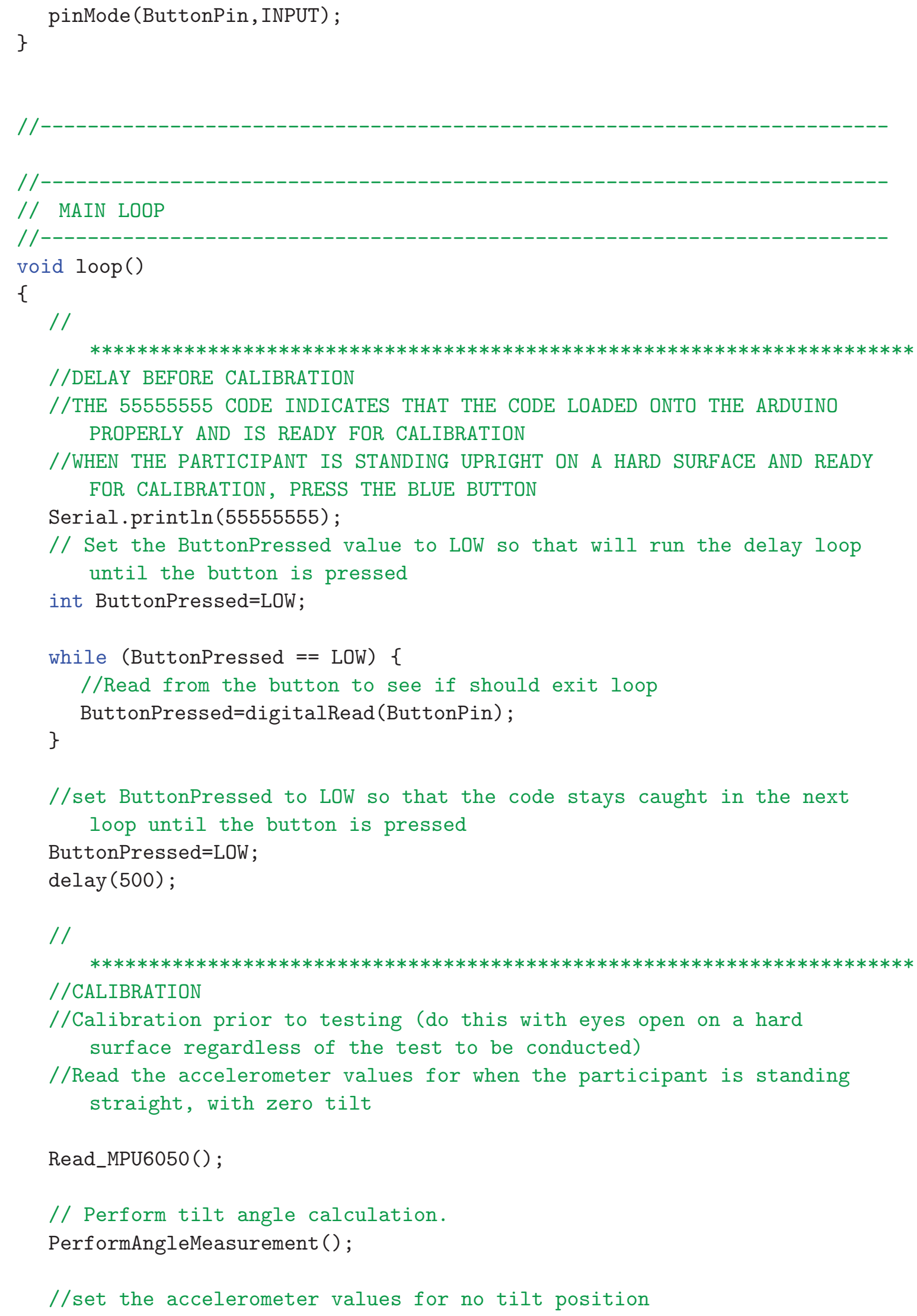


AccelTiltAngleZero_X=AccelTiltangle_X;

AccelTiltAngleZero_Y=AccelTiltAngle_Y;

Serial.println(66666666); // indicates that the calibration is done //

***************************************************************************

//ALLOW TIME FOR PARTICIPANT TO SET-UP

//the 66666666 code indicates that calibration is done; the participant should get into the position

//to be tested (eyes open or closed on foam or a hard surface) and then the blue button should be

//pressed once again before the test begins

//

//there will be no feedback given in this time, but the tilt angles still need to be measured,

//otherwise the large time difference between measurements will cause large errors in tilt measurement

while (ButtonPressed == LOW) \{

// Read and calculate the angles

Read_MPU6050();

PerformAngleMeasurement();

//Read from the button to see if should exit loop

\}

ButtonPressed=digitalRead (ButtonPin);

//

$* * * * * * * * * * * * * * * * * * * * * * * * * * * * * * * * * * * * * * * * * * * * * * * * * * * * * * * * * * * * * * * * * * * * * *$

// TESTING

//tilt is constantly measured -- if the tilt exceeds 3 degrees in the M/L or $A / P$ direction,

// the corresponding tactor is activated with low intensity. If it exceeds 5 degrees in either

// direction, the corresponding tactor is activated with high intensity

//

//only one tactor is activated at a time, in the direction with the greatest tilt magnitude

for (long $i=0 ; i<$ TestLength; $i++$ ) \{

// Collect accel. and gyro. measurements from the IMU chip.

//Serial.print ("1*");

Read_MPU6050();

// Perform tilt angle calculation.

//Serial.print("2*"); 
PerformAngleMeasurement ();

//Serial.println("3*");

// check if angle is outside the 3 or 5 degree thresholds and provide proper feedback

// Relating Tilt Angle to Tactors

// the IMU should be mounted on the participant's back using the 3 D printed holding box

$/ /$ for trunk tilt in the anterior direction, the tilt angle in the $y$ direction will be positive,

// for trunk tilt in the posterior direction, the tilt angle in the $y$ direction will be negative

// for trunk tilt in the M/L direction to the right, the tilt angle in the $\mathrm{x}$ direction will be positive

// for trunk tilt in the M/L direction to the left, the tilt angle in the $\mathrm{x}$ direction will be negative

//

// Tactor 1 : placed on the front of the body

// Tactor 2 : placed on the right of the body

// Tactor 3 : placed on the back of the body

// Tactor 4 : placed on the left of the body

//**** turn off all the tactors before you turn on a new one!!!!

// we want the tactor in the direction of greatest tilt to vibrate (because only one tactor can vibrate at a time)

if (abs (TiltAngle_X) >abs (TiltAngle_Y ) $\{$ //give feedback about the $\mathrm{X}$ axis in the M/L direction (tactors 2 and 4 )

if (TiltAngle_X $>0$ ) \{

$/ /$ if tilt is greater than $0 \rightarrow$ tilting to the right

if (TiltAngle_X $>3$ ) \{

// vibrate Tactor 2 on high and Tactor 4 is off

digitalWrite(Tactor1Pin, LOW);

digitalWrite(Tactor3Pin, LOW);

digitalWrite(Tactor4Pin, LOW);

delay (TimeDelay);

analogWrite(Tactor2Pin, $\mathrm{H}$ );

\} else if (TiltAngle_X $>1$ ) $\{/ /$ and $<5$

// vibrate Tactor 2 on low and Tactor 4 is off

digitalWrite(Tactor1Pin, LOW);

digitalWrite(Tactor3Pin, LOW);

digitalWrite(Tactor4Pin, LOW);

delay (TimeDelay);

analogWrite(Tactor2Pin, L);

\} else $\{/ /$ in a balanced state

digitalWrite(Tactor1Pin, LOW); 


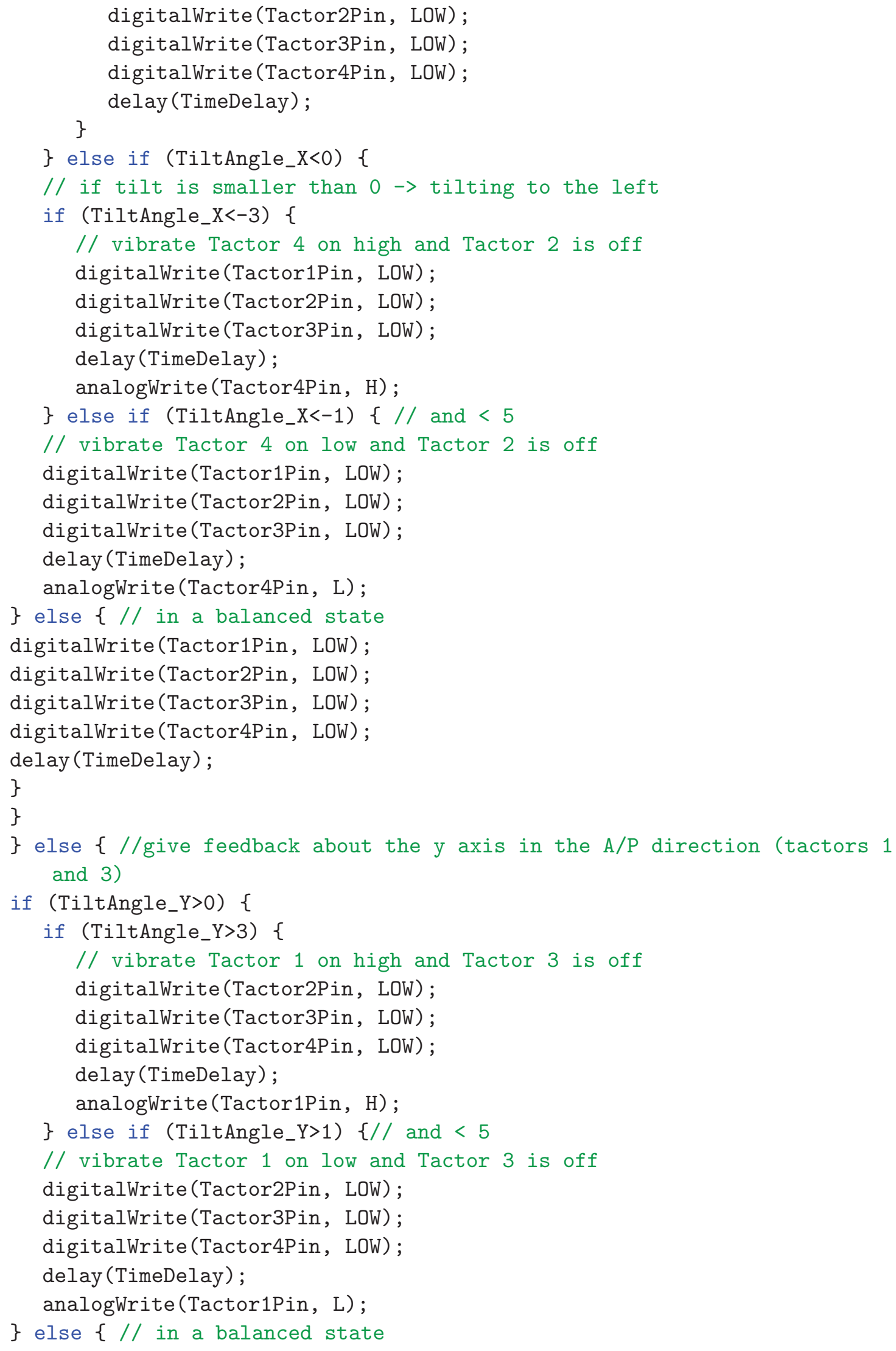




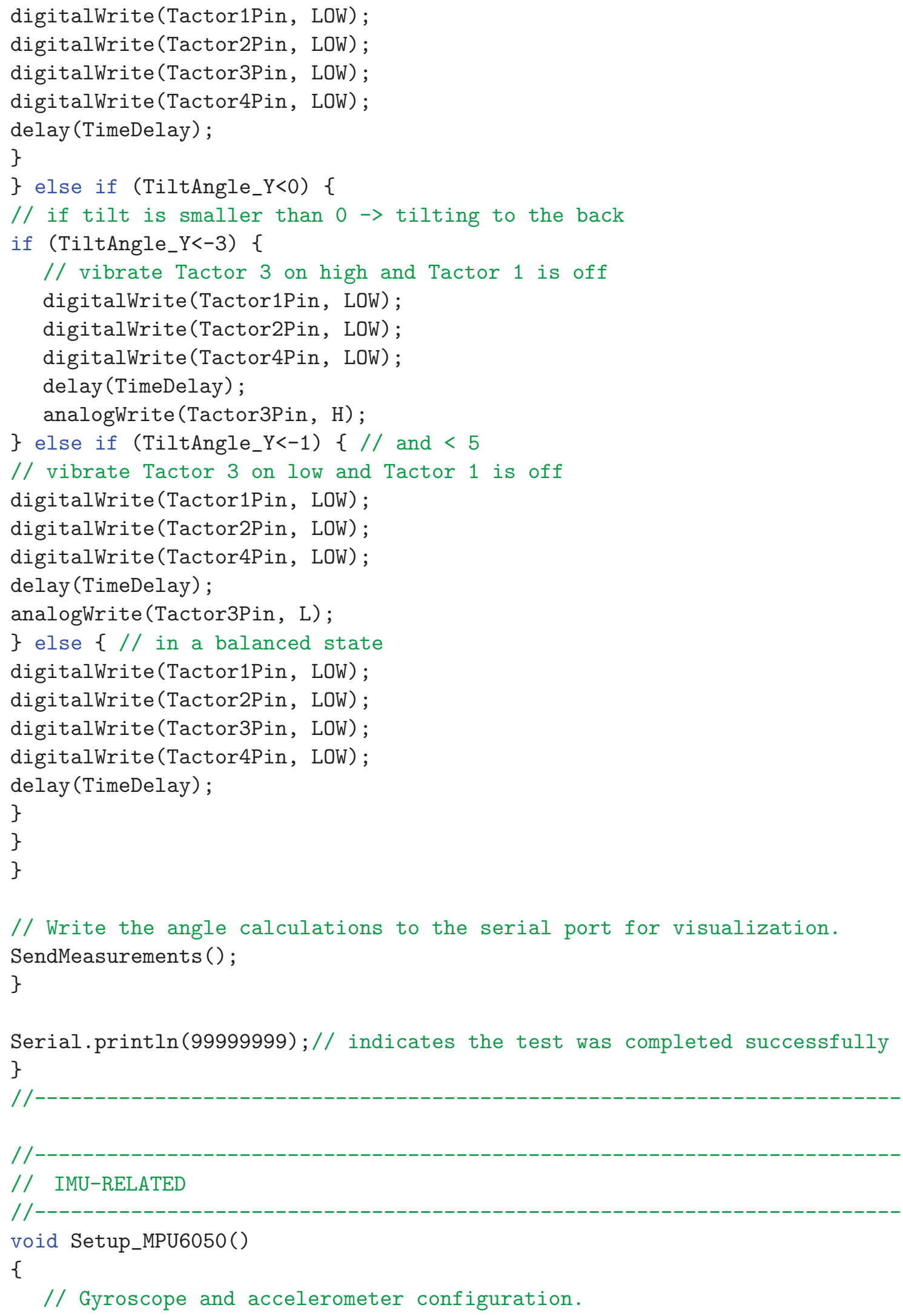




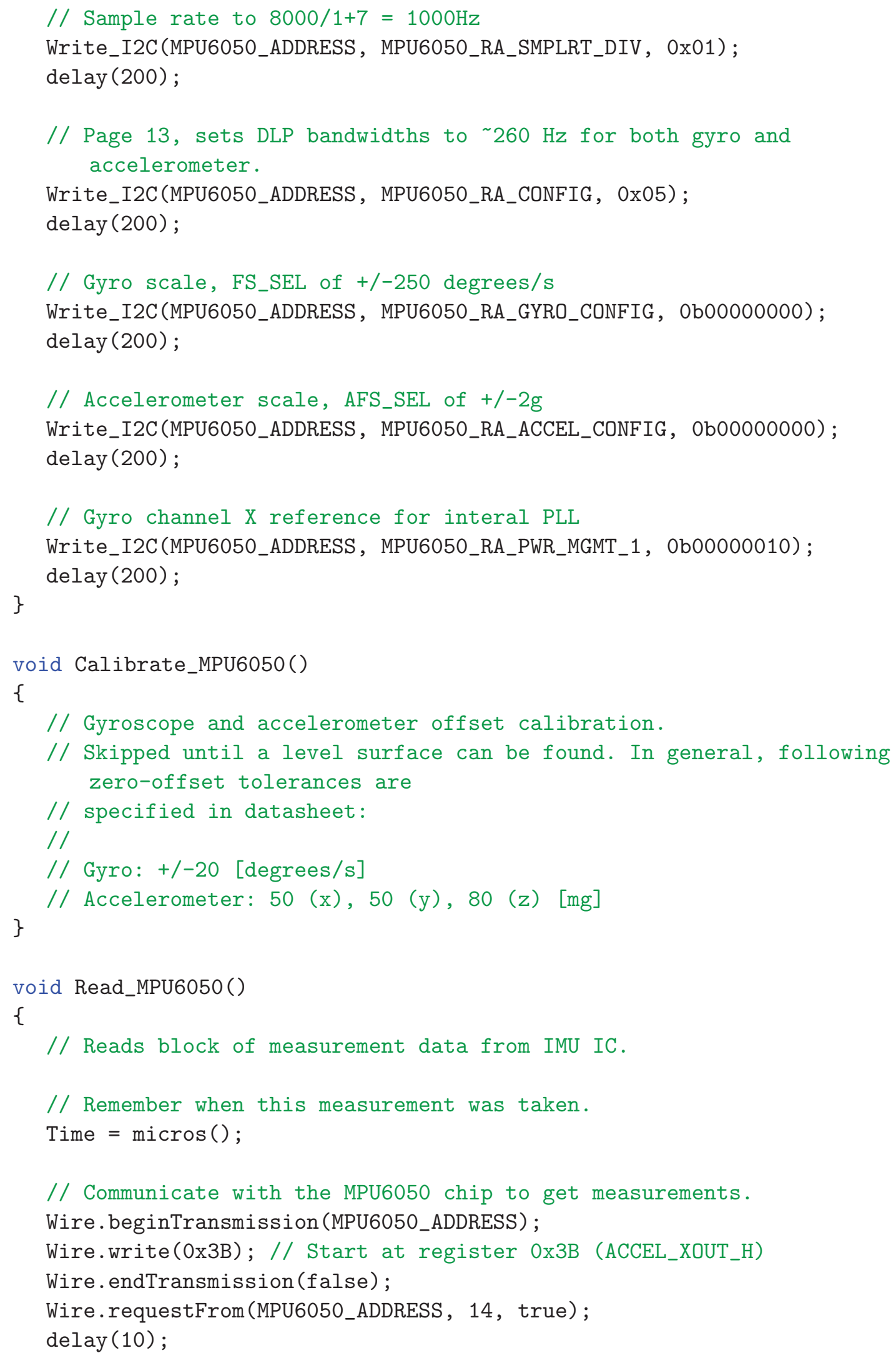




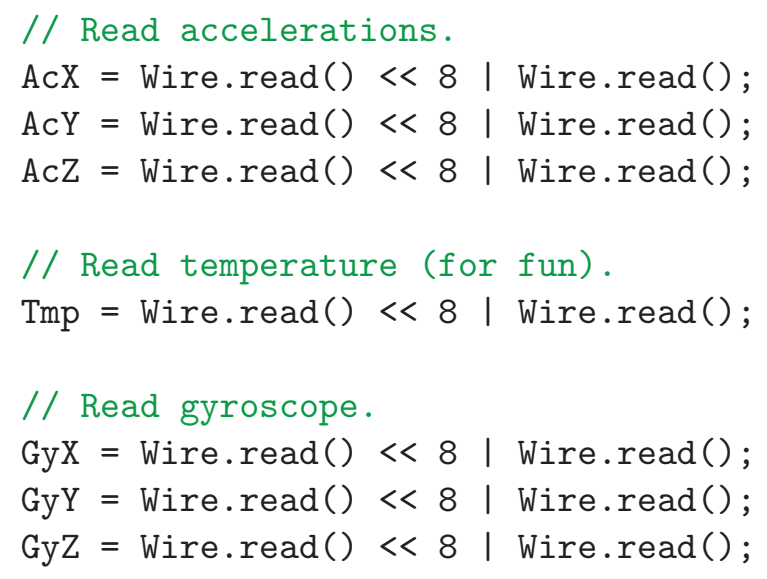


\}

$1 /$

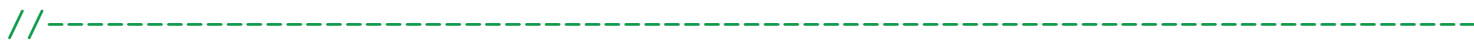

// TILT-RELATED

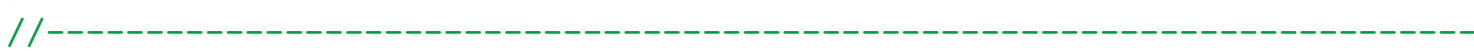

void PerformAngleMeasurement()

\{

// This function uses the raw accelerometer and gyroscope measurements and creates a tilt estimate.

//--------------------------------------------------------------------

// 0 . Measurement Conversion

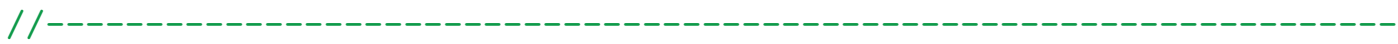

// Here we measurements from bits to useable units

// In case you are curious about how the accel. / gyro. ranges imply a particular sensitivity:

// These are all from the MPU6050 datasheet, btw.

// Gyro range [deg/s] | Sensitivity

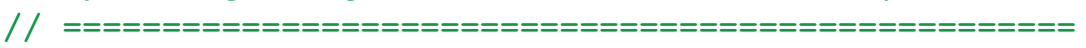

// 250 | 131

// $500 \quad$ | 65.5

// $1000 \quad$ | 32.8

// $2000 \quad$ | 16.4

// Accel range [g] | Sensitivity

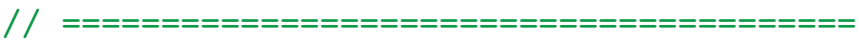

// 2 | 16384

// 4 | 8192

// $8 \quad$ | 4096

// $16 \quad$ | 2048

// Based on the accel. range selected, this is how you convert bits to g (where $\left.1 \mathrm{~g}=9.81 \mathrm{~m} / \mathrm{s}^{\wedge} 2\right)$

float AcX_G = (float $(A c X)) / 16384.0$;

float AcY_G = (float $(\mathrm{AcY})) / 16384.0$;

float AcZ_G = (float $(A c Z)) / 16384.0$;

// Based on gyro. range selected, this is how you convert bits to degrees/s

float GyX_Deg = float $(\mathrm{GyX}) / 131.0$;

float GyY_Deg = float $(\mathrm{GyY}) / 131.0$;

float GyZ_Deg = float $($ Gyz) / 131.0;

$/ /$

// 1. Gyroscope Tilt Angle Estimation 
//------------------------------------------------------------------

// First get the particular angular velocity measurement that we care about (the axis about which

// the tilt will be happening). This angle is also called the roll angle (beta).

// In this case, I use $Z$ axis.

float AngularAcceleration_X = GyX_Deg;

float AngularAcceleration_Y = GyY_Deg;

// Determine the time elapsed since last measurement, delta $t$.

unsigned long ElapsedTime = Time - PreviousTime;

// We record our new time measurement for future.

PreviousTime $=$ Time;

// Use our particular angular velocity measurement to get the tilt angle through integration:

// Basically angular velocity [degrees/s] * delta $t$ [s] $=$ [degrees]

// Note the $+=$ symbol, we are adding incrementally to previous measurement.

float DeltaGyroAngle_X = AngularAcceleration_X $*$ ElapsedTime $* 0.000001$; GyroTiltAngle_X += DeltaGyroAngle_X;

float DeltaGyroAngle_Y $=$ AngularAcceleration_Y $*$ ElapsedTime $* 0.000001$; GyroTiltAngle_Y += DeltaGyroAngle_Y;

/// 2. Accelerometer Tilt Angle Estimation

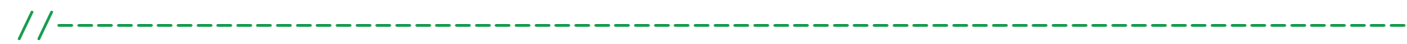

// We measure roll angle (beta) in relation to the $\mathrm{Y}$ axis. Based on the FBD with three acceleration

// vectors, we can calculate the tilt angle using trig. To get a better idea of what is going on,

// have a look at following PDF, specifically Equation 9 on page 13.

//http://www.st.com/content/ccc/resource/technical/document/applicatio

//n_note/8e/28/c0/ea/1f/ed/4e/48/CD00268887.pdf/files/CD00268887.pdf/j

//cr: content/translations/en.CD00268887.pdf

// Also convert from radians to degrees.

AccelTiltAngle_X $=\left(\operatorname{atan} 2\left(A_{-} Y_{-} G, \operatorname{sqrt}\left(A_{c} X_{-} G * A_{-} X_{-} G+A_{-} Z_{-} * A_{c} Z_{-} G\right)\right) *\right.$ 180.0) / PI;

AccelTiltAngle_X=AccelTiltAngle_X-AccelTiltAngleZero_X;

AccelTiltAngle_Y $=-\left(\operatorname{atan} 2\left(A_{-} X_{-} G, \operatorname{sqrt}\left(A_{c Y} Y_{-} * A_{-} Y_{-} G+A_{-} Z_{-} * A_{c} Z_{-} G\right)\right)\right.$

* 180.0) / PI;

AccelTiltAngle_Y=AccelTiltAngle_Y-AccelTiltAngleZero_Y;

// The accelerometer reading is quite noisy as it picks up minute accelerations. You will see

// this on the visualization. To combat this, a simple low-pass filter is used before fusion. 


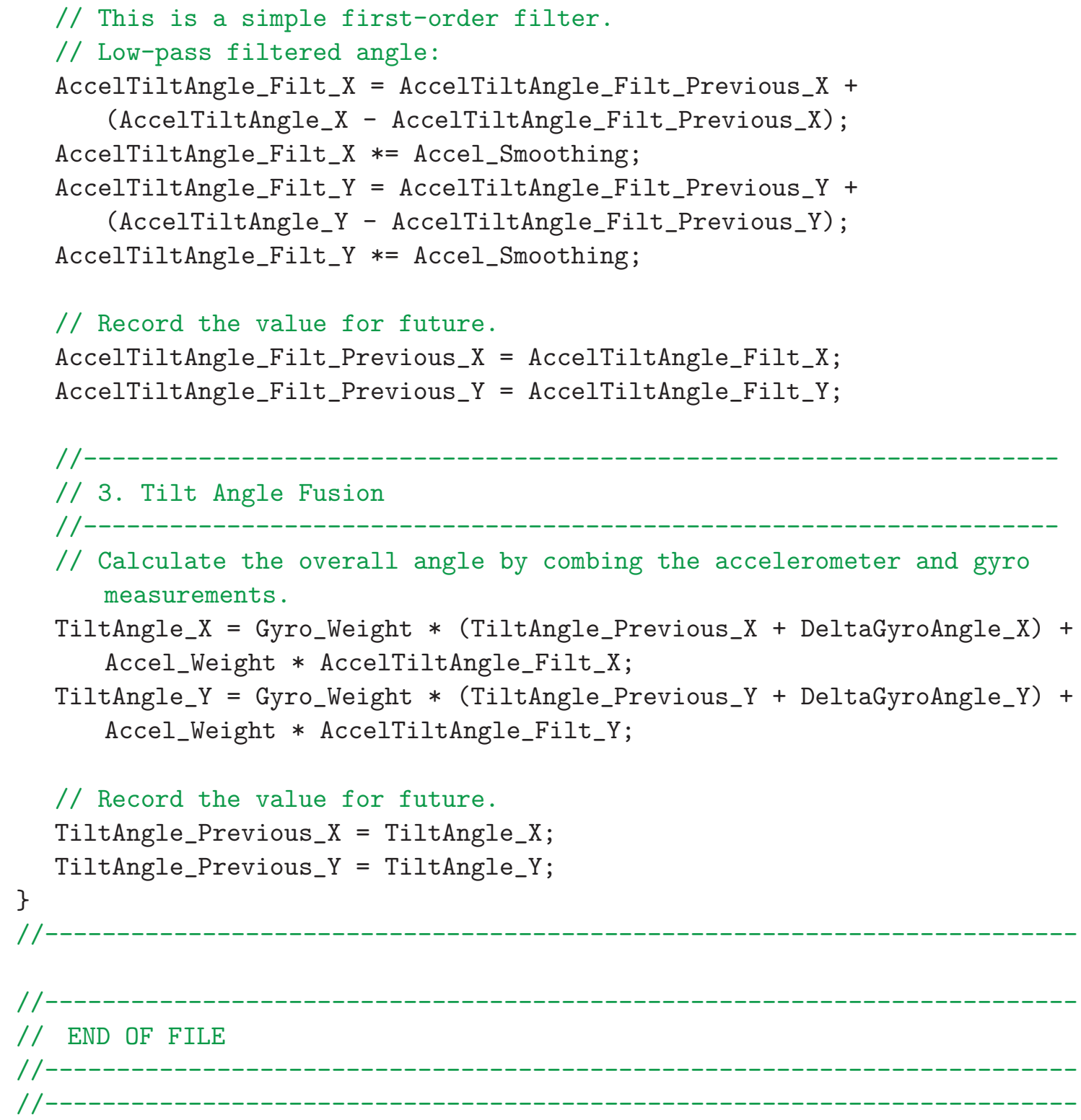


Appendix I

Swaypaths for Individual Participants 

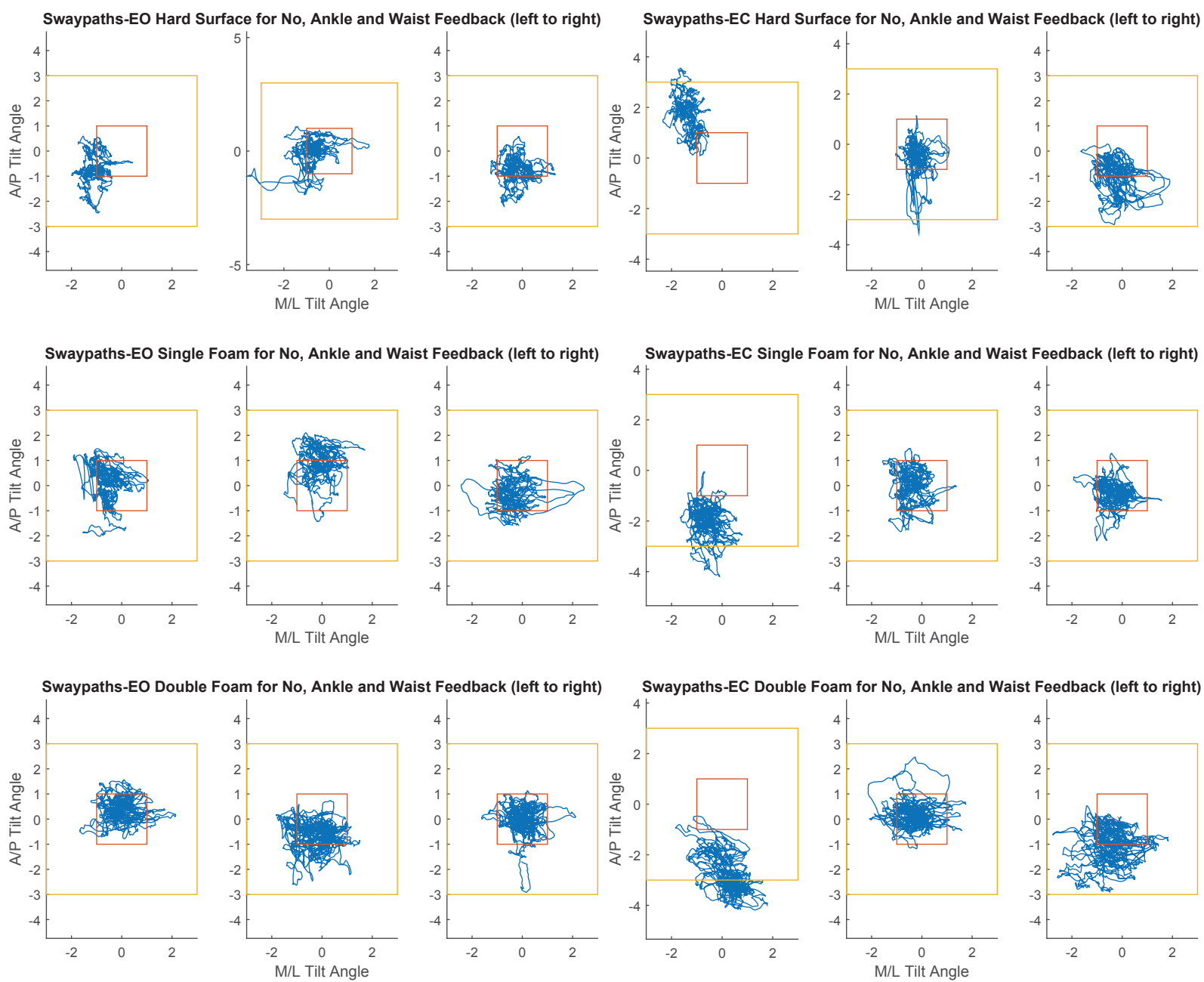

Figure I.1: Participant 1's swaypath for all tests performed. 

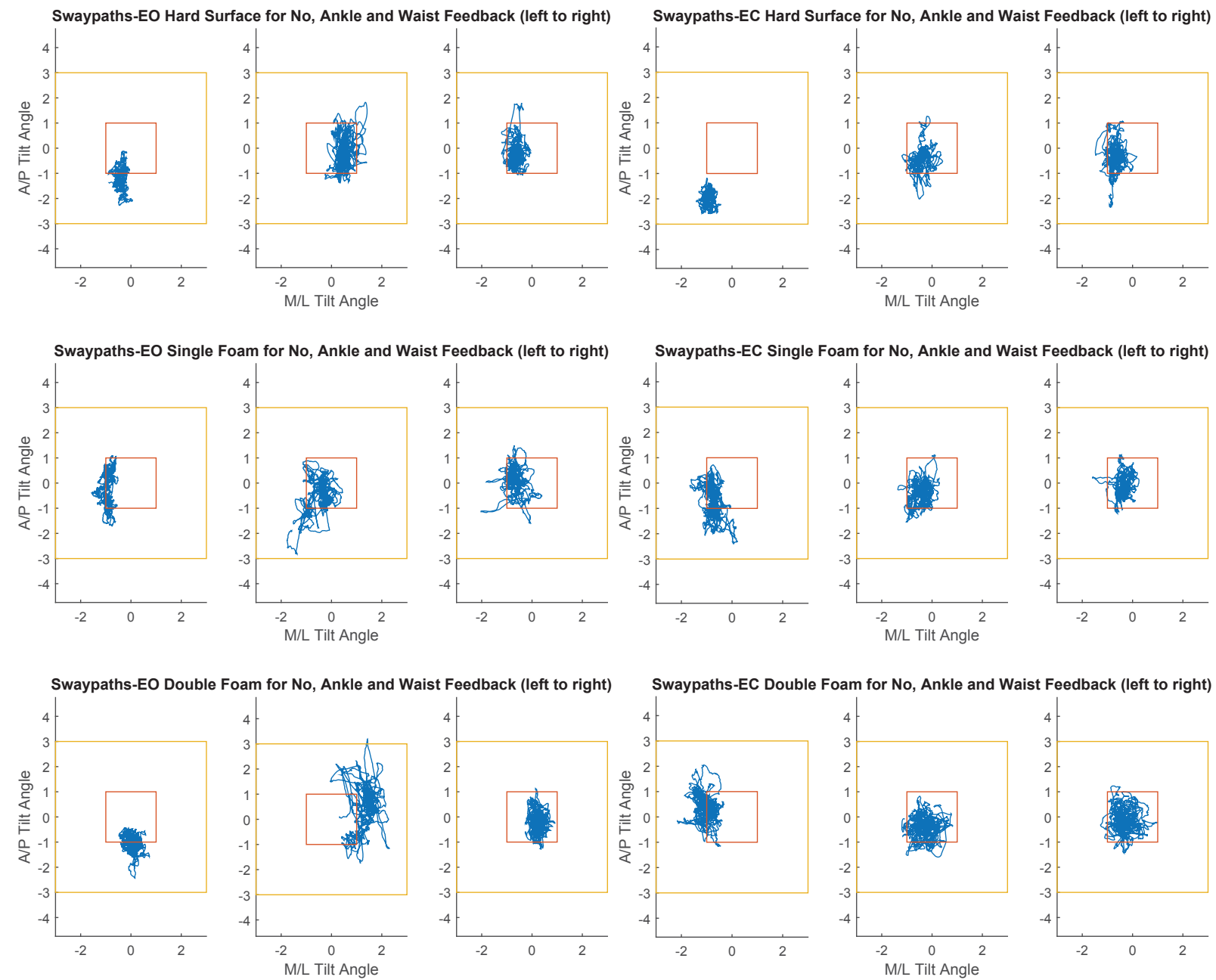

Figure I.2: Participant 2's swaypath for all tests performed. 

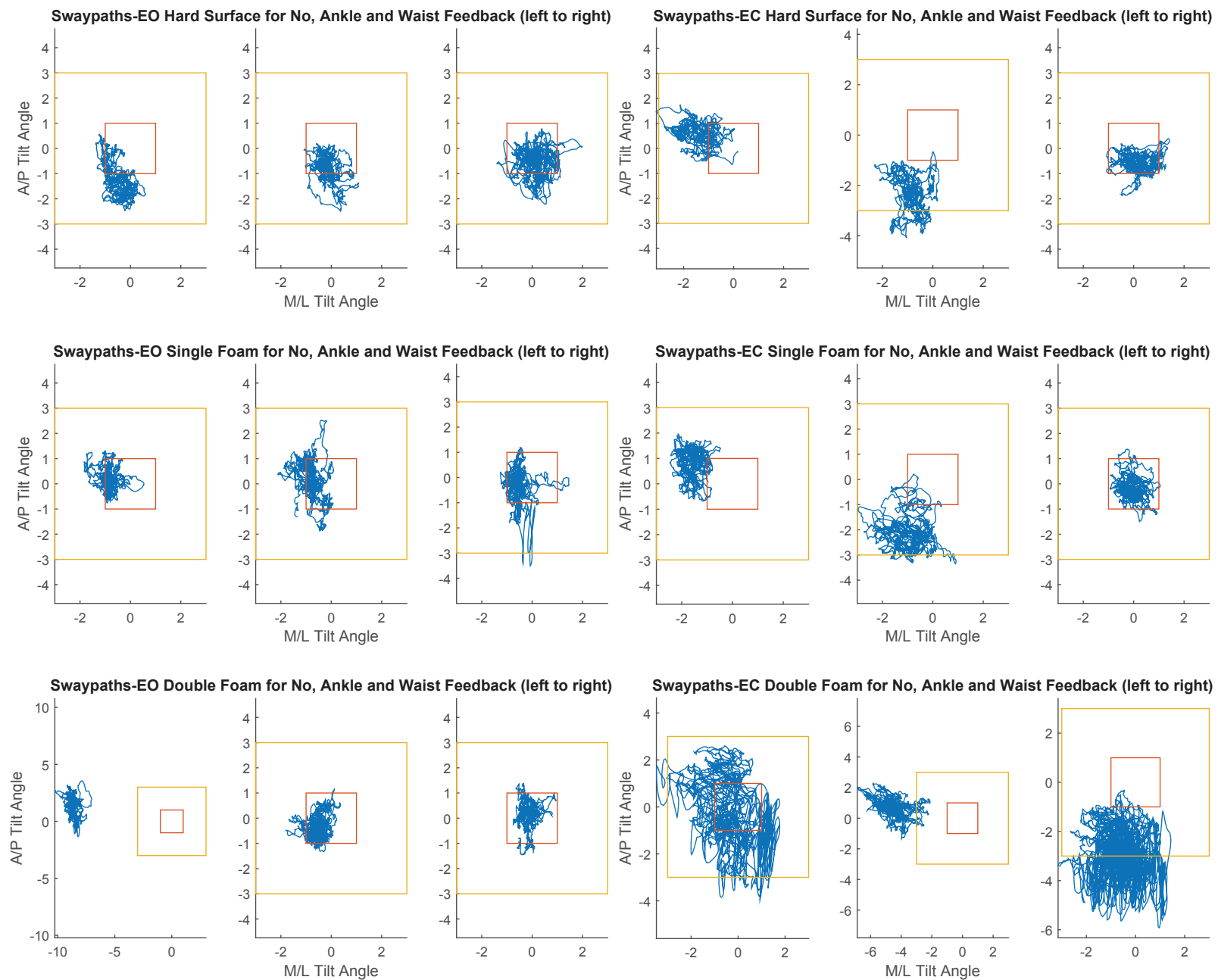

Figure I.3: Participant 3's swaypath for all tests performed. 

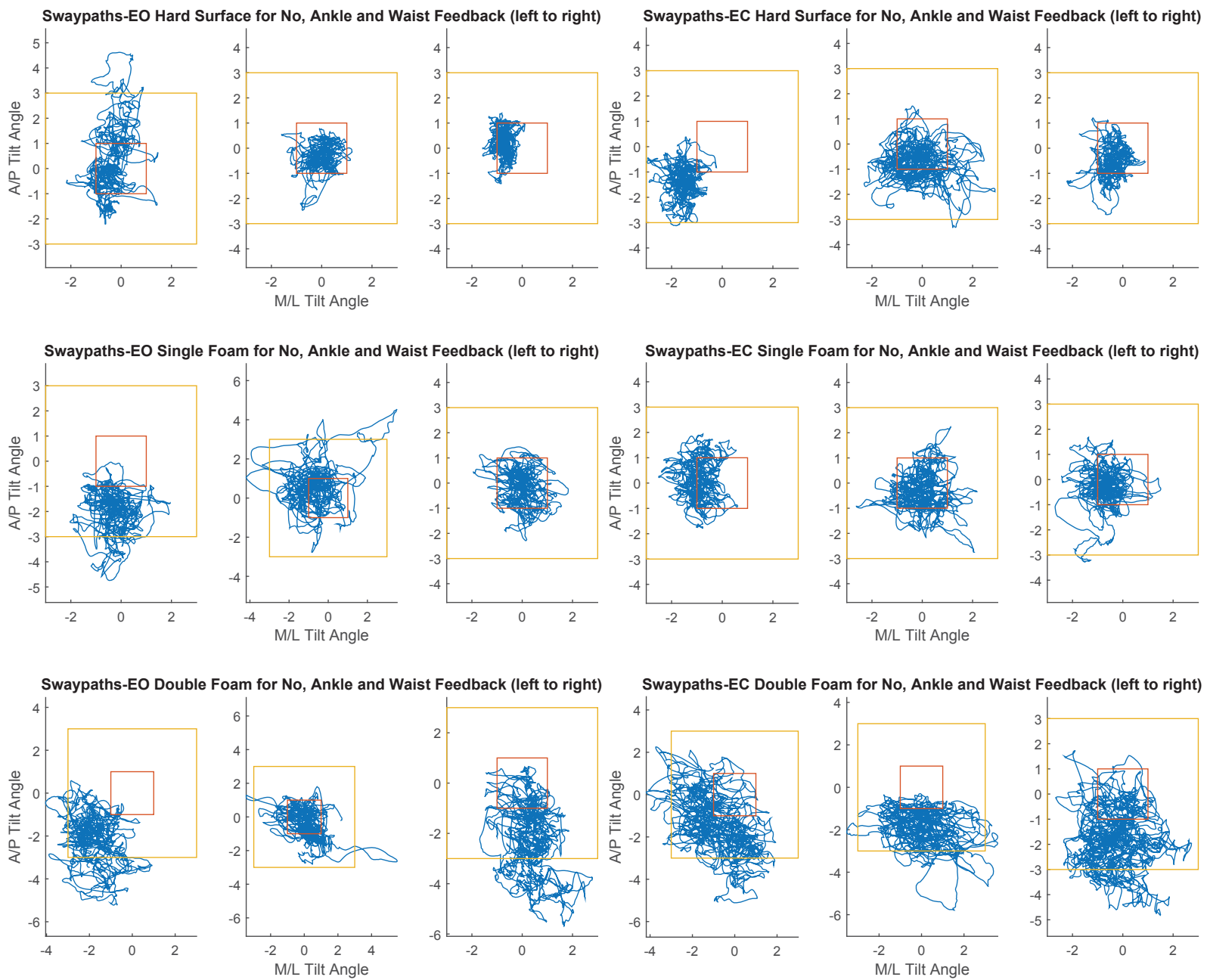

Figure I.4: Participant 4's swaypath for all tests performed. 

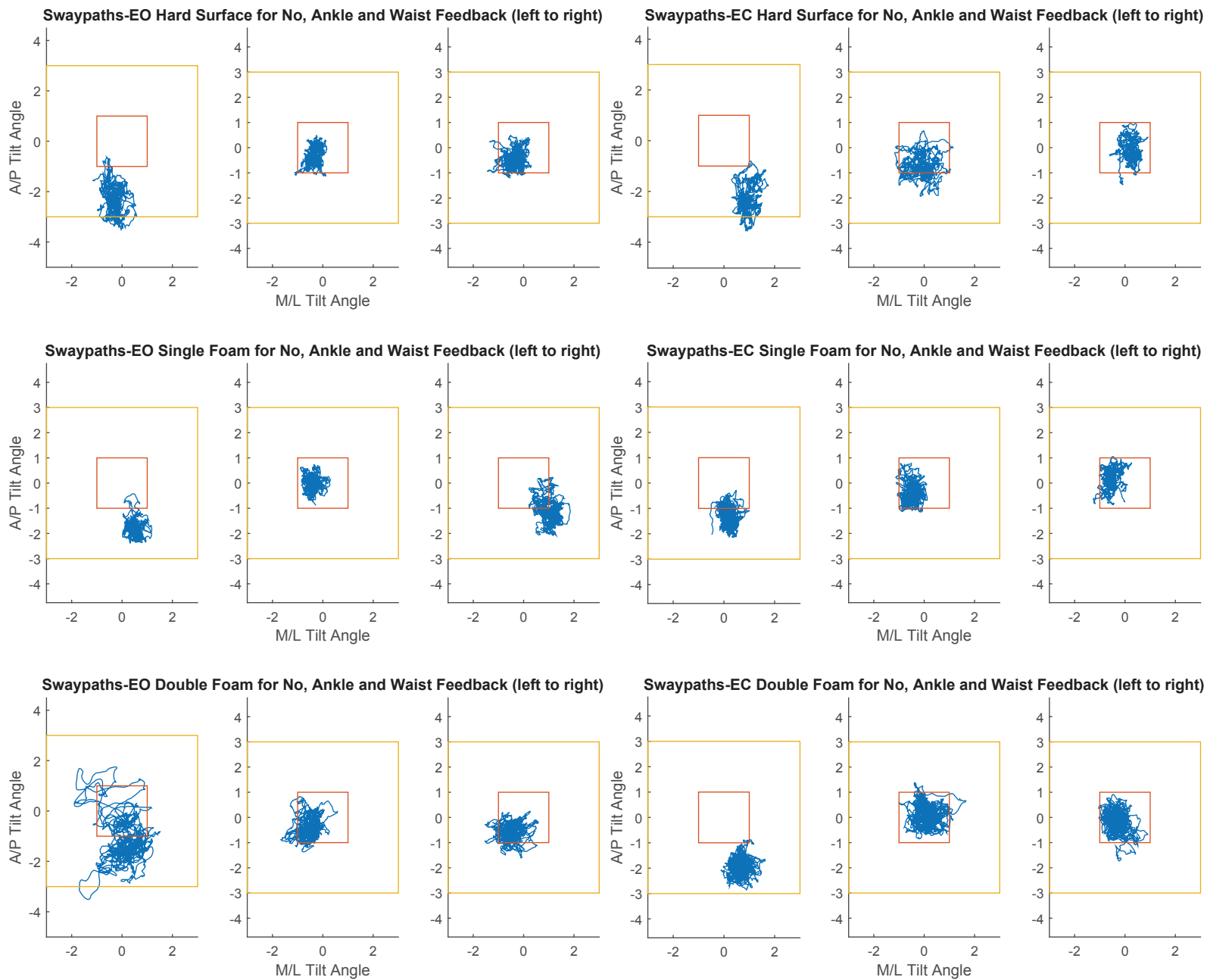

Figure I.5: Participant 5's swaypath for all tests performed. 

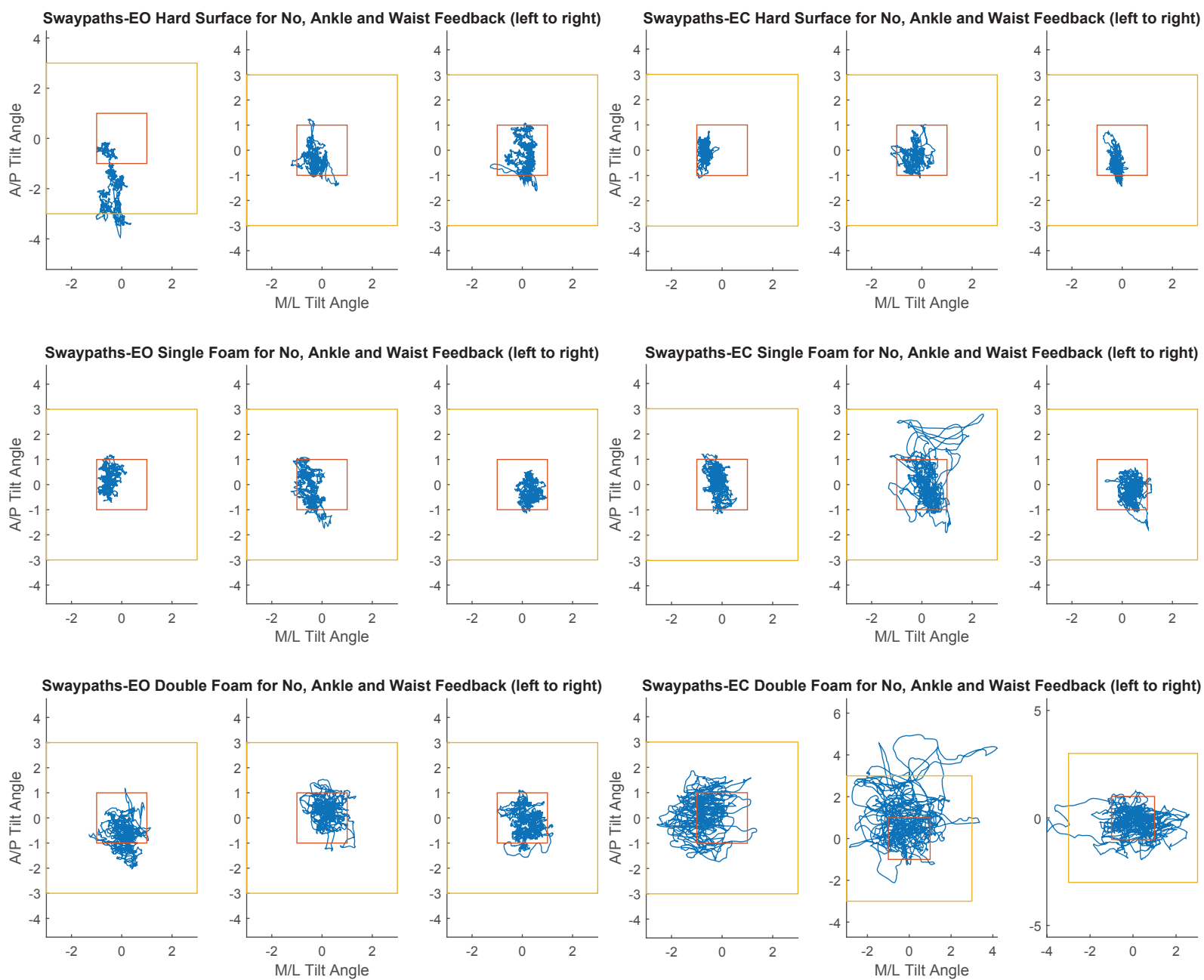

Figure I.6: Participant 6's swaypath for all tests performed. 

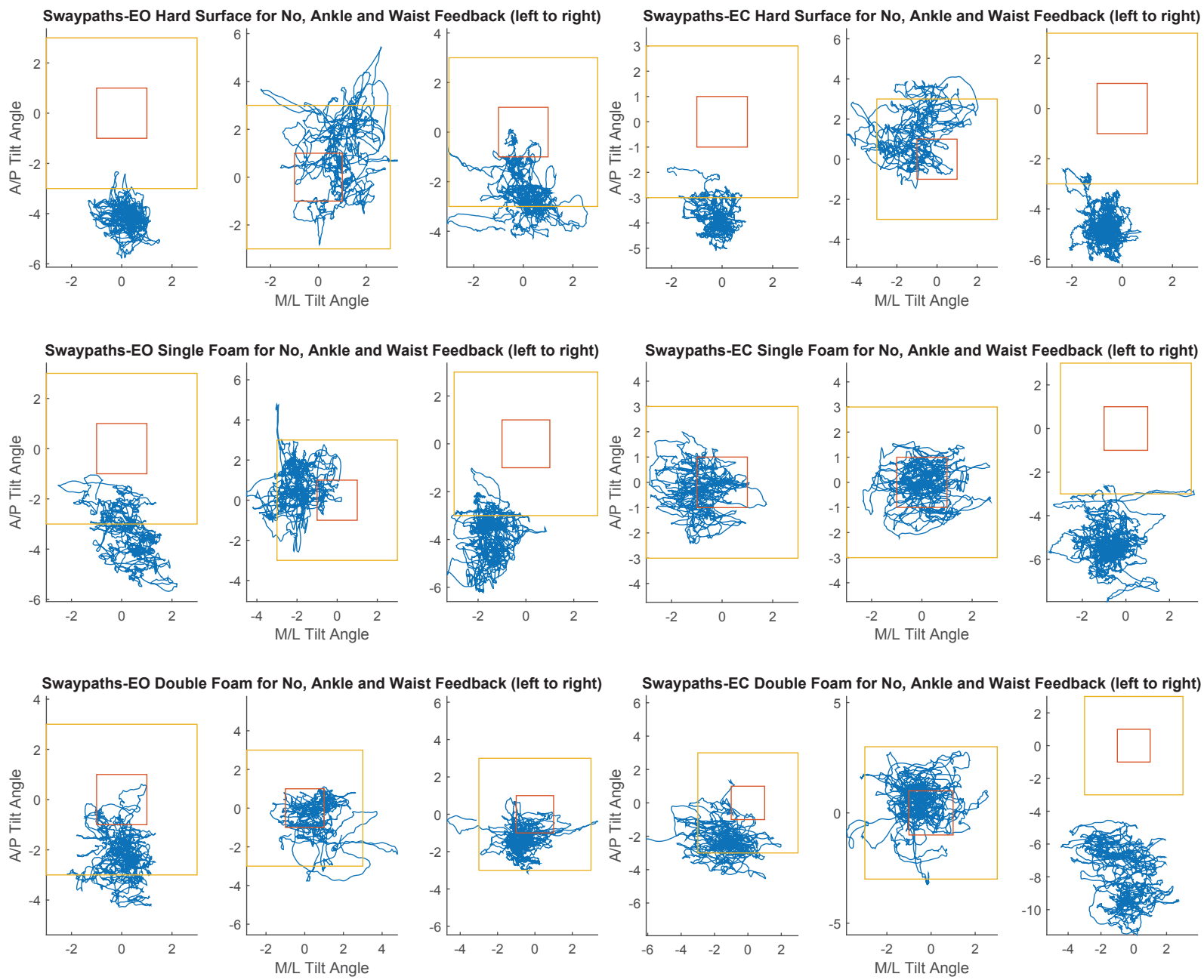

Figure I.7: Participant 7's swaypath for all tests performed. 

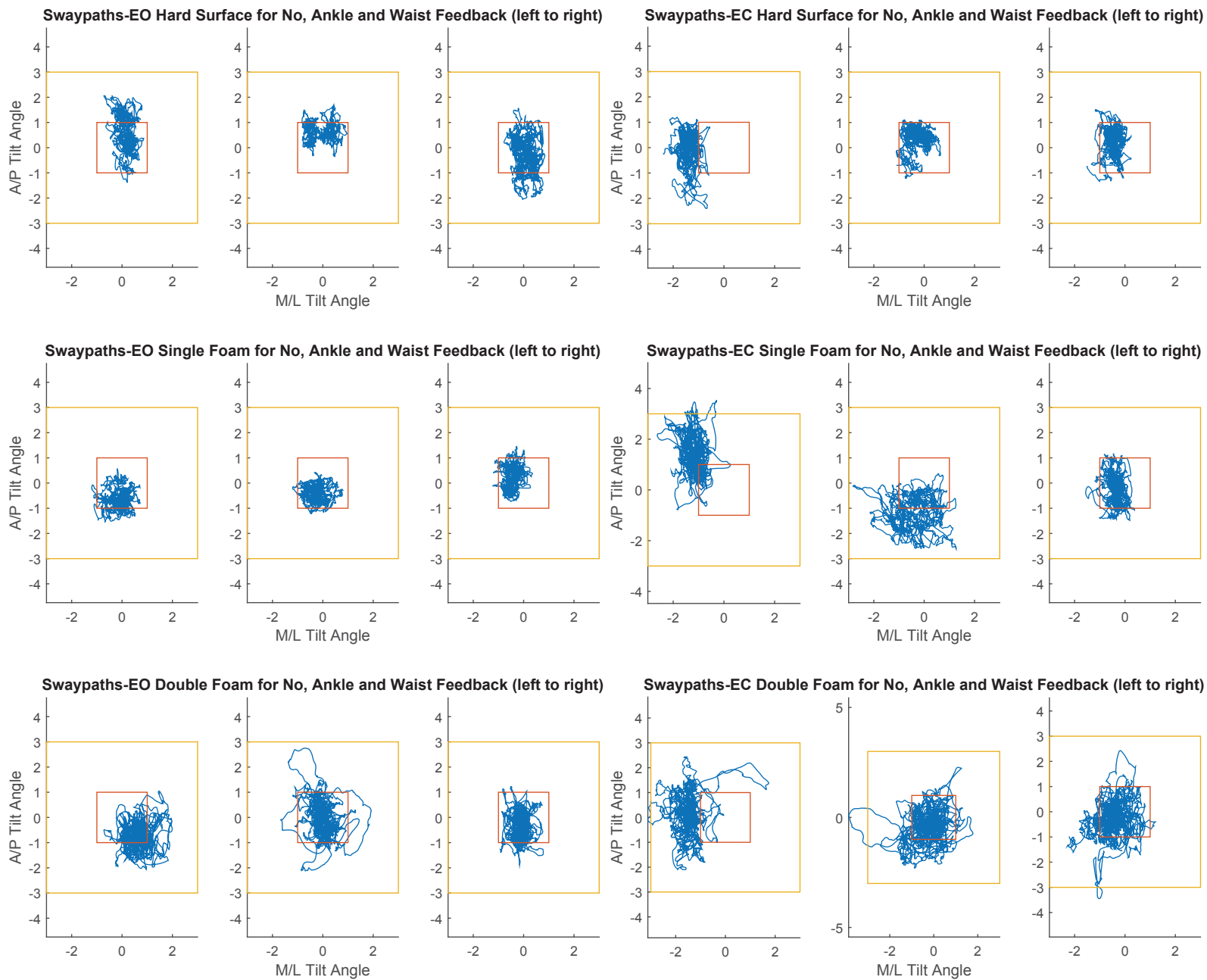

Figure I.8: Participant 8's swaypath for all tests performed. 

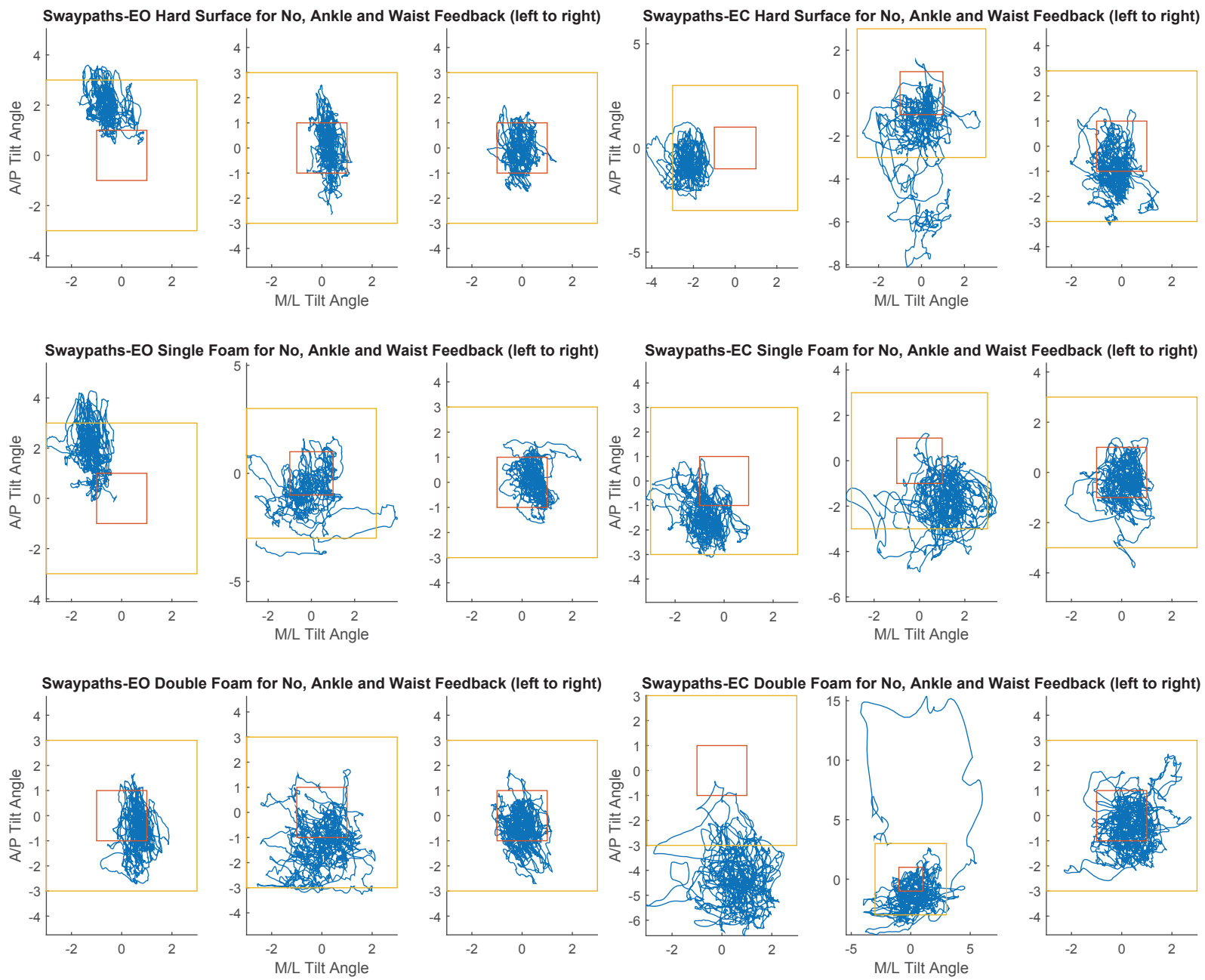

Figure I.9: Participant 9's swaypath for all tests performed. 

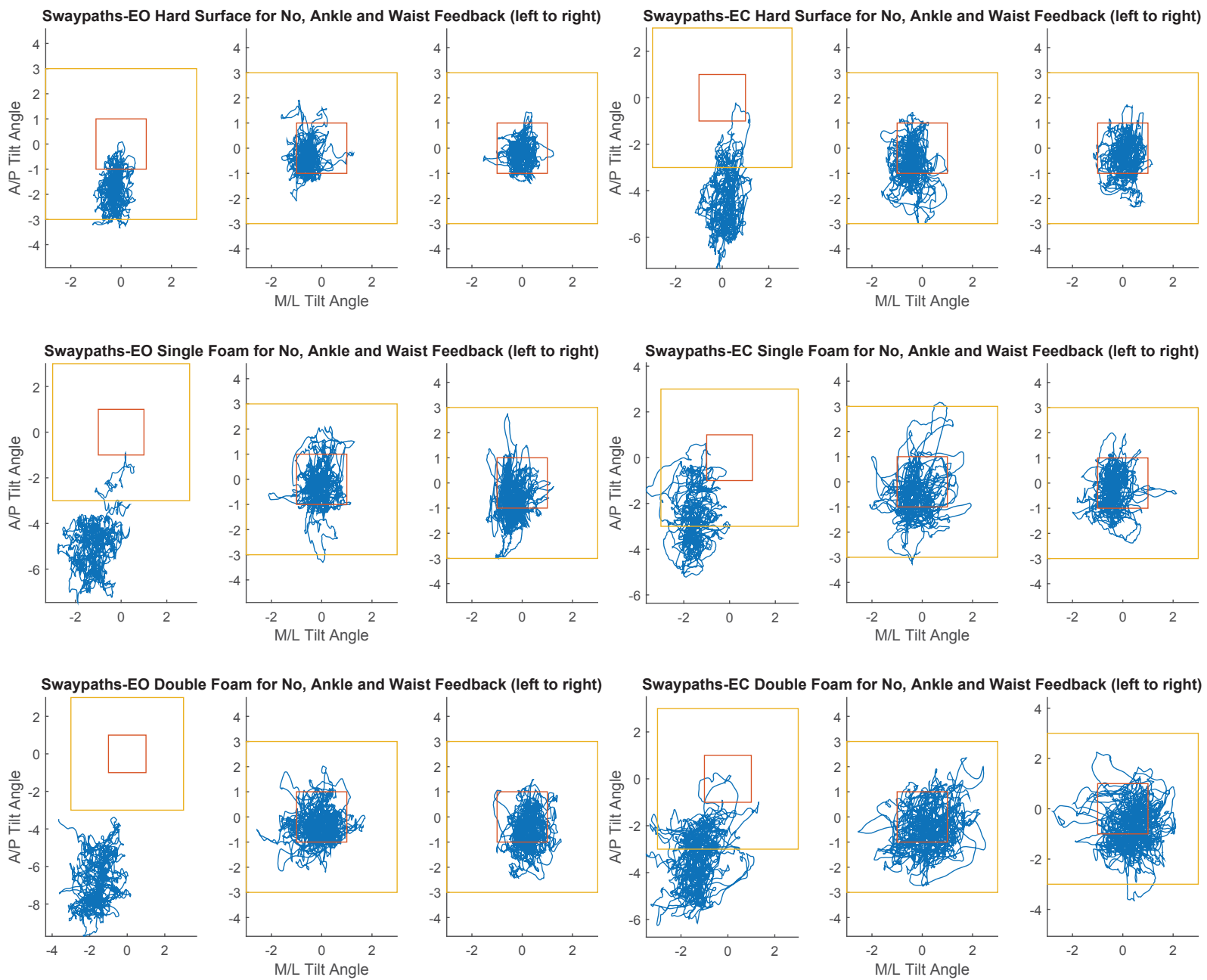

Figure I.10: Participant 10's swaypath for all tests performed. 

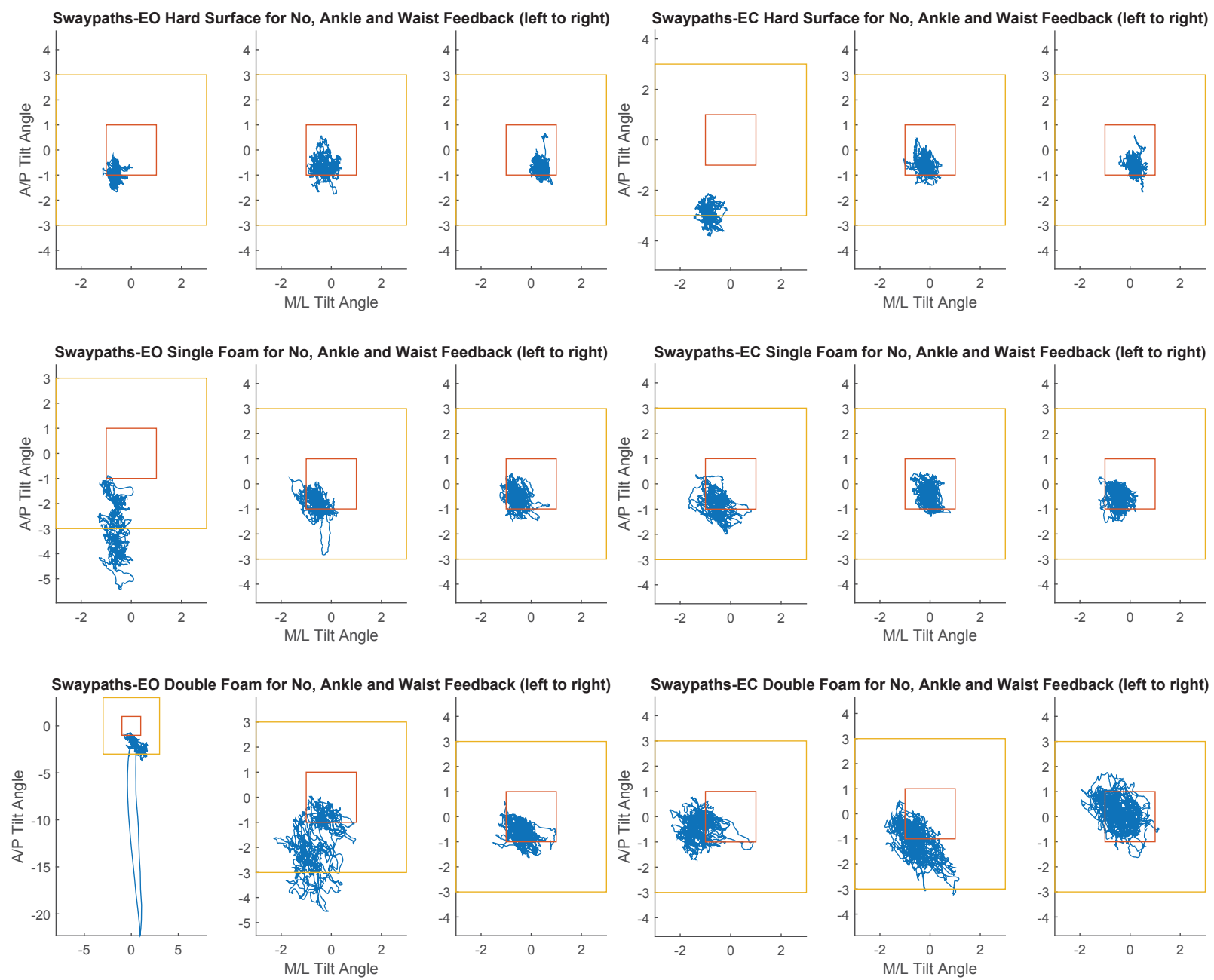

Figure I.11: Participant 11's swaypath for all tests performed. 

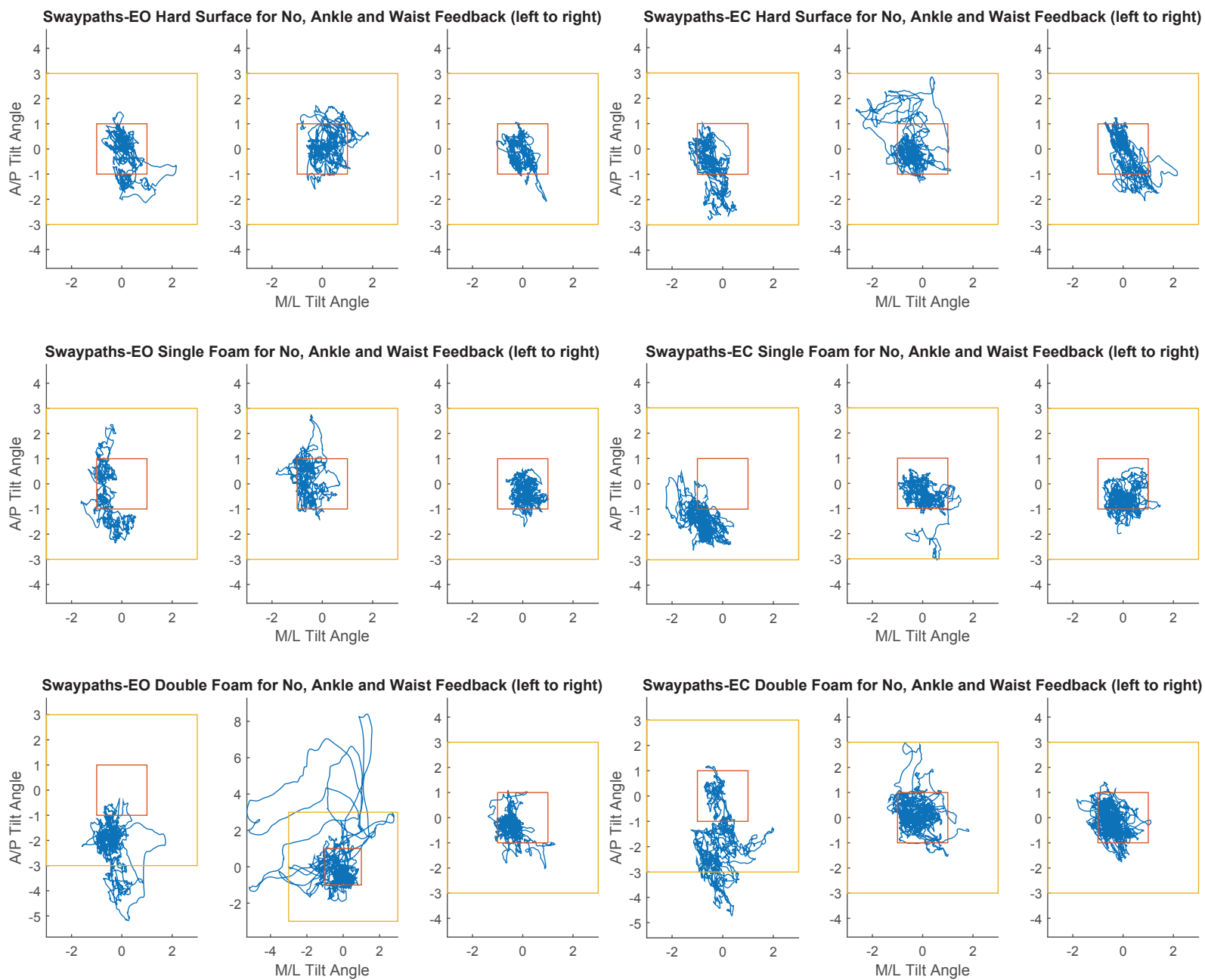

Figure I.12: Participant 12's swaypath for all tests performed. 


\section{Appendix J}

\section{Significance of Difference Between \\ Feedback Type Performance on}

\section{Evaluation Criteria}

The following values were obtained by conducting individual repeated measures ANOVAs with a Greenhouse-Geisser correction for each of the six Trials. The presence of a significant difference between at least two of the three feedback types on an individual measure is represented by a $\mathrm{p}$ value smaller than 0.05 .

Table J.1: Significance of ML tilt differences found using the Repeated Measures ANOVA

\begin{tabular}{lll}
\hline Trial & $\mathbf{F}$ & $\mathbf{p}$ value \\
\hline Trial 1 & .849 & .433 \\
Trial 2 & 8.907 & $\mathbf{. 0 0 8}$ \\
Trial 3 & .418 & .620 \\
Trial 4 & 4.617 & $\mathbf{. 0 2 4}$ \\
Trial 5 & 1.466 & .252 \\
Trial 6 & 1.011 & .344 \\
\hline
\end{tabular}


Table J.2: Significance of AP tilt differences found using the Repeated Measures ANOVA

\begin{tabular}{lll}
\hline Trial & $\mathbf{F}$ & $\mathbf{p}$ value \\
\hline Trial 1 & 21.085 & $\mathbf{. 0 0 0}$ \\
Trial 2 & 2.949 & .081 \\
Trial 3 & 4.999 & $\mathbf{. 0 2 4}$ \\
Trial 4 & .475 & .566 \\
Trial 5 & 4.494 & $\mathbf{. 0 4 5}$ \\
Trial 6 & 1.214 & .308 \\
\hline
\end{tabular}

Table J.3: Significance of reaction time differences found using the Repeated Measures ANOVA

\begin{tabular}{lll}
\hline Trial & $\mathbf{F}$ & $\mathbf{p}$ value \\
\hline Trial 1 & 3.431 & .091 \\
Trial 2 & 5.765 & $\mathbf{. 0 1 6}$ \\
Trial 3 & 3.558 & 0.054 \\
Trial 4 & .487 & .539 \\
Trial 5 & 4.236 & .064 \\
Trial 6 & .041 & .954 \\
\hline
\end{tabular}

Table J.4: Significance of percentage time spent in the deadzone differences found using the Repeated Measures ANOVA

\begin{tabular}{lll}
\hline Trial & $\mathbf{F}$ & $\mathbf{p}$ value \\
\hline Trial 1 & 44.013 & $\mathbf{. 0 0 0}$ \\
Trial 2 & 19.154 & $\mathbf{. 0 0 0}$ \\
Trial 3 & 8.311 & $\mathbf{. 0 0 3}$ \\
Trial 4 & 10.077 & $\mathbf{. 0 0 1}$ \\
Trial 5 & 10.667 & $\mathbf{. 0 0 1}$ \\
Trial 6 & 9.628 & $\mathbf{. 0 0 2}$ \\
\hline
\end{tabular}


Table J.5: Significance of dominant motion frequency differences found using the Repeated Measures ANOVA

\begin{tabular}{lll}
\hline Trial & $\mathbf{F}$ & $\mathbf{p}$ value \\
\hline Trial 1 & .373 & .690 \\
Trial 2 & .529 & .592 \\
Trial 3 & 1.670 & .222 \\
Trial 4 & .065 & .934 \\
Trial 5 & .140 & .819 \\
Trial 6 & .159 & .853 \\
\hline
\end{tabular}




\section{Appendix K}

\section{Means, Standard Deviations and $P$ values for Each of the Tests}

The significance of pairwise comparisons was found through conducting post hoc paired-samples t-tests using the Sidak correction for multiple comparisons. A p value smaller than 0.05 indicates a statistically significant difference between the two feedback types compared.

For the RMS AP tilt, as found through the repeated measures ANOVAs conducted, only Trials 1, 3 and 5 present significant differences between at least two feedback conditions and merit further investigation through post hoc analysis. For completeness, $\mathrm{p}$ values for pairwise comparisons on all trials are provided, but only those relating to Trials 1,3 and 5 are of interest.

Table K.1: RMS AP tilt

\begin{tabular}{ccccccc}
\hline Test & \multicolumn{3}{c}{ Mean(SD) } & & \multicolumn{3}{c}{ P Values } \\
& No Feedback & Ankle & Waist & $\begin{array}{c}\text { No/Ankle } \\
\text { Comparison }\end{array}$ & $\begin{array}{c}\text { No/Waist } \\
\text { Comparison }\end{array}$ & $\begin{array}{c}\text { Ankle/Waist } \\
\text { Comparison }\end{array}$ \\
\hline Trial 1 & $1.69(0.94)$ & $0.74(0.34)$ & $0.78(0.57)$ & $\mathbf{0 . 0 0 3}$ & $\mathbf{0 . 0 0 1}$ & 0.953 \\
\hline Trial 2 & $1.92(1.34)$ & $1.12(0.73)$ & $1.06(1.18)$ & 0.276 & 0.149 & 0.998 \\
\hline Trial 3 & $1.84(1.47)$ & $0.83(0.31)$ & $0.91(0.96)$ & 0.089 & 0.093 & 0.987 \\
\hline Trial 4 & $1.33(0.66)$ & $1.00(0.59)$ & $0.98(1.37)$ & 0.499 & 0.854 & 1.000 \\
\hline Trial 5 & $2.00(1.71)$ & $0.97(0.45)$ & $0.83(0.58)$ & 0.193 & 0.103 & 0.902 \\
\hline Trial 6 & $1.97(1.25)$ & $1.08(0.63)$ & $1.62(2.16)$ & 0.085 & 0.943 & 0.801 \\
\hline
\end{tabular}


For the RMS ML tilt, as found through the repeated measures ANOVAs conducted, only Trials 2 and 4 present significant differences between at least two feedback conditions and merit further investigation through post hoc analysis. For completeness, $\mathrm{p}$ values for pairwise comparisons on all trials are provided, but only those relating to Trials 2 and 4 are of interest. It can be found in Table K.2 that for Trial 5, a p value smaller than $0.05(\mathrm{p}=.029)$ exists for the ankle/wrist comparison. However, this is a spurious significant difference, since the repeated measures ANOVA found no significant differences for Trial 5. Therefore this difference is not considered significant and is indicated as so in the RMS ML tilt plot in Chapter 5.

Table K.2: RMS ML tilt

\begin{tabular}{ccccccc}
\hline Test & \multicolumn{3}{c}{ Mean(SD) } & & \multicolumn{3}{c}{ P Values } \\
& No Feedback & Ankle & Waist & $\begin{array}{c}\text { No/Ankle } \\
\text { Comparison }\end{array}$ & $\begin{array}{c}\text { No/Waist } \\
\text { Comparison }\end{array}$ & $\begin{array}{c}\text { Ankle/Waist } \\
\text { Comparison }\end{array}$ \\
\hline Trial 1 & $0.55(0.23)$ & $0.57(0.27)$ & $0.47(0.16)$ & 0.999 & 0.616 & 0.527 \\
\hline Trial 2 & $1.10(0.54)$ & $0.65(0.34)$ & $0.50(0.18)$ & 0.113 & $\mathbf{0 . 0 0 8}$ & 0.166 \\
\hline Trial 3 & $0.77(0.28)$ & $0.78(0.48)$ & $0.68(0.34)$ & 1.000 & 0.836 & 0.669 \\
\hline Trial 4 & $0.93(0.43)$ & $0.69(0.33)$ & $0.57(0.22)$ & 0.158 & 0.064 & 0.673 \\
\hline Trial 5 & $1.46(2.35)$ & $0.77(0.27)$ & $0.51(0.22)$ & 0.722 & 0.488 & 0.029 \\
\hline Trial 6 & $1.12(0.41)$ & $1.13(1.12)$ & $0.79(0.25)$ & 1.000 & 0.029 & 0.626 \\
\hline
\end{tabular}

For the dominant movement frequency, as found through the repeated measures ANOVAs conducted, no Trial presents significant differences between at least two feedback conditions and merits further investigation through post hoc analysis. For completeness, $\mathrm{p}$ values for pairwise comparisons on all trials are provided, but none are actually of interest.

For the reaction time, as found through the repeated measures ANOVAs conducted, only Trial 2 presents significant differences between at least two feedback conditions and merits further investigation through post hoc analysis. For completeness, p values for pairwise comparisons on all trials are provided, but only those relating to Trial 2 are of interest. The lack of significance in differences after post 
Table K.3: Dominant Movement Frequency

\begin{tabular}{ccccccc}
\hline Test & \multicolumn{3}{c}{ Mean(SD) } & & \multicolumn{3}{c}{ P Values } \\
No Feedback & Ankle & Waist & $\begin{array}{c}\text { No/Ankle } \\
\text { Comparison }\end{array}$ & $\begin{array}{c}\text { No/Waist } \\
\text { Comparison }\end{array}$ & $\begin{array}{c}\text { Ankle/Waist } \\
\text { Comparison }\end{array}$ \\
\hline Trial 1 & $0.20(0.07)$ & $0.19(0.06)$ & $0.21(0.07)$ & 0.994 & 0.927 & 0.777 \\
\hline Trial 2 & $0.22(0.07)$ & $0.23(0.08)$ & $0.20(0.04)$ & 0.925 & 0.969 & 0.658 \\
\hline Trial 3 & $0.21(0.05)$ & $0.20(0.06)$ & $0.26(0.13)$ & 0.957 & 0.528 & 0.480 \\
\hline Trial 4 & $0.24(0.09)$ & $0.23(0.10)$ & $0.23(0.08)$ & 0.985 & 0.985 & 1.000 \\
\hline Trial 5 & $0.21(0.06)$ & $0.23(0.07)$ & $0.21(0.08)$ & 0.966 & 0.999 & 0.971 \\
\hline Trial 6 & $0.28(0.11)$ & $0.31(0.09)$ & $0.30(0.12)$ & 0.932 & 0.972 & 0.999 \\
\hline
\end{tabular}

Table K.4: Reaction Time

\begin{tabular}{ccccccc}
\hline Test & \multicolumn{3}{c}{ Mean(SD) } & & \multicolumn{3}{c}{ P Values } \\
No Feedback & Ankle & Waist & $\begin{array}{c}\text { No/Ankle } \\
\text { Comparison }\end{array}$ & $\begin{array}{l}\text { No/Waist } \\
\text { Comparison }\end{array}$ & $\begin{array}{c}\text { Ankle/Waist } \\
\text { Comparison }\end{array}$ \\
\hline Trial 1 & $3.92(12.44)$ & $1.06(2.45)$ & $0.85(4.77)$ & 0.260 & 0.234 & 0.860 \\
\hline Trial 2 & $11.77(27.57)$ & $1.46(4.49)$ & $1.11(6.82)$ & 0.076 & 0.051 & 0.981 \\
\hline Trial 3 & $4.54(17.05)$ & $1.38(3.32)$ & $1.17(7.15)$ & 0.113 & 0.190 & 0.732 \\
\hline Trial 4 & $3.22(9.27)$ & $1.41(4.63)$ & $1.00(7.07)$ & 0.412 & 0.999 & 0.799 \\
\hline Trial 5 & $3.80(13.56)$ & $1.00(2.75)$ & $0.89(2.92)$ & 0.187 & 0.173 & 0.812 \\
\hline Trial 6 & $3.53(11.61)$ & $1.47(5.92)$ & $1.26(6.30)$ & 0.990 & 0.994 & 1.000 \\
\hline
\end{tabular}

hoc analysis may indicate that the study lacked statistical power even though the reaction time's effect size was included in the estimation of the minimal sample size.

For the percentage time in the deadzone, as found through the repeated measures ANOVAs conducted, all Trials present significant differences between at least two feedback conditions and merit further investigation through post hoc analysis. Through post-hoc analysis, statistically significant differences between at least two means for each Trial were identified. 
Table K.5: Percent Time in the Deadzone

\begin{tabular}{ccccccc}
\hline Test & \multicolumn{3}{c}{ Mean(SD) } & & \multicolumn{3}{c}{ P Values } \\
& No Feedback & Ankle & Waist & $\begin{array}{c}\text { No/Ankle } \\
\text { Comparison }\end{array}$ & $\begin{array}{l}\text { No/Waist } \\
\text { Comparison }\end{array}$ & $\begin{array}{c}\text { Ankle/Waist } \\
\text { Comparison }\end{array}$ \\
\hline Trial 1 & $0.31(0.24)$ & $0.80(0.21)$ & $0.83(0.24)$ & $\mathbf{0 . 0 0 0}$ & $\mathbf{0 . 0 0 0}$ & 0.662 \\
\hline Trial 2 & $0.17(0.32)$ & $0.65(0.33)$ & $0.77(0.28)$ & $\mathbf{0 . 0 0 5}$ & $\mathbf{0 . 0 0 0}$ & 0.466 \\
\hline Trial 3 & $0.38(0.39)$ & $0.67(0.27)$ & $0.76(0.30)$ & 0.057 & $\mathbf{0 . 0 0 6}$ & 0.771 \\
\hline Trial 4 & $0.30(0.31)$ & $0.65(0.33)$ & $0.81(0.28)$ & $\mathbf{0 . 0 1 5}$ & $\mathbf{0 . 0 0 6}$ & 0.528 \\
\hline Trial 5 & $0.30(0.32)$ & $0.63(0.27)$ & $0.78(0.27)$ & 0.056 & $\mathbf{0 . 0 0 1}$ & 0.464 \\
\hline Trial 6 & $0.17(0.19)$ & $0.52(0.34)$ & $0.57(0.35)$ & $\mathbf{0 . 0 3 3}$ & $\mathbf{0 . 0 0 7}$ & 0.878 \\
\hline
\end{tabular}




\section{List of References}

[1] World Health Organization, "Falls." http://www.who.int/violence_injury_ prevention/other_injury/falls/en/. [Online; accessed December 10, 2016].

[2] Canadian Patient Safety Institute, "Falls." http://www. patientsafetyinstitute.ca/en/Topic/Pages/Falls.aspx. [Online; accessed December 1, 2016].

[3] "Seniors' falls in canada- second report."

[4] "The cost of injury in canada," June 2015.

[5] C. Ma, D. Wong, W. Lam, A. Wan, and W. Lee, "Balance improvement effects of biofeedback systems with state-of-the-art wearable sensors: A systematic review," Sensors, vol. 16, April 2016.

[6] K. Sienko, M. Balkwill, L. Oddsson, and C. Wall, "Effects of multi-directional vibrotactile feedback on vestibular-deficient postural performance during continuous multi-directional support surface perturbations," Journal of Vestibular Research: Equilibrium Éamp; Orientation, vol. 18, no. 5-6, 2008.

[7] P.-Y. Lee, K. Gadareh, M. J. Naushahi, M. Gresty, and A. M. Bronstein, "Protective stepping response in Parkinsonian patients and the effect of vibrotactile feedback.," Movement disorders : official journal of the Movement Disorder Society, vol. 28, pp. 482-489, apr 2013.

[8] N. Bugnariu and J. Fung, "Aging and selective sensorimotor strategies in the regulation of upright balance," Journal of Neuroengineering Rehabilitation, vol. 4, pp. 1-7, 2007.

[9] J. Hegeman, F. Honegger, M. Kupper, and J. Allum, "The balance control of bilateral peripheral vestibular loss subjects and its improvement with auditory prosthetic feedback," Journal of Vestibular Research, vol. 15, pp. 109-17, 2005. 
[10] I. I. I. Wall C., M. S. Weinberg, P. B. Schmidt, D. E. Krebs, C. Wall III, M. S. Weinberg, P. B. Schmidt, D. E. Krebs, C. Wall, M. S. Weinberg, P. B. Schmidt, and D. E. Krebs, "Balance prosthesis based on micromechanical sensors using vibrotactile feedback of tilt," IEEE Trans. Biomed. Eng. (USA), vol. 48, pp. 1153 -61, oct 2001.

[11] E. Walker, A. Hernandez, and M. Kattan, "Meta-analysis: Its strengths and limitations," Cleveland Clinic Journal of Medicine, vol. 75, pp. 431-9, June 2008.

[12] M. Ebell and H. Barry, "Reading a meta-analysis." Online.

[13] M. Ebell and H. Barry, "Methods fo continuous outcomes." http://omerad. msu.edu/ebm/Meta-analysis/meth_cont_outcome.html. [Online; accessed November 30, 2016].

[14] Cochrane Collaboration, "Obtainning standard deviations from standard errors, confidence intervals, t values and p values for differences in means." http://handbook. cochrane.org/chapter_7/7_7_3_3_obtaining_standard_ deviations_from_standard_errors.htm, January 2015.

[15] J. R. Davis, M. G. Carpenter, R. Tschanz, S. Meyes, D. Debrunner, J. Burger, and J. H. J. Allum, "Trunk sway reductions in young and older adults using multi-modal biofeedback.," Gait $\&$ posture, vol. 31, pp. 465-72, apr 2010.

[16] L. L. Verhoeff, C. G. C. Horlings, L. J. F. Janssen, S. A. Bridenbaugh, and J. H. J. Allum, "Effects of biofeedback on trunk sway during dual tasking in the healthy young and elderly," Gait \& Posture, vol. 30, no. 1, pp. 76-81, 2009.

[17] C. Wall, E. Kentala, I. I. I. Wall C., and E. Kentala, "Control of sway using vibrotactile feedback of body tilt in patients with moderate and severe postural control deficits," J. Vestib. Res., Equilib. Orientat. (Netherlands), vol. 15, no. 56, pp. $313-25,2005$.

[18] C. r. Wall, D. M. Wrisley, K. D. Statler, C. Wall III, D. M. Wrisley, and K. D. Statler, "Vibrotactile tilt feedback improves dynamic gait index: A fall risk indicator in older adults," GAIT ES POSTURE, vol. 30, pp. 16-21, jul 2009.

[19] M. Janssen, R. Stokroos, J. Aarts, R. van Lummel, and H. Kingma, "Salient and placebo vibrotactile feedback are equally effective in reducing sway in bilateral vestibular loss patients.," Gait \& posture, vol. 31, pp. 213-7, feb 2010.

[20] R. Peterka, "Sensorimotor integration in human postural control," Journal of Neurophysiology, vol. 88, pp. 1097-1118, 2002. 
[21] R. Peterka, C. Wall, and E. Kentala, "Determining the effectiveness of a vibrotactile balance prosthesis," Journal of Vestibular Research: Equilibrium Gamp; Orientation, vol. 16, no. 1-2, 2006.

[22] K. H. K. Sienko, V. V. Vichare, M. D. Balkwill, C. Wall, and C. Wall III, "Assessment of vibrotactile feedback on postural stability during pseudorandom multidirectional platform motion," IEEE Transactions on Biomedical Engineering, vol. 57, pp. 944-952, apr 2010.

[23] A. Goodworth, C. Wall, and R. Peterka, "A balance control model predicts how vestibular loss subjects benefit from a vibrotactile balance prosthesis," 2011 33rd Annual International Conference of the IEEE Engineering in Medicine and Biology Society, 2011.

[24] S. Haggerty, L.-T. Jiang, A. Galecki, and K. H. Sienko, "Effects of biofeedback on secondary-task response time and postural stability in older adults.," Gait \&6 posture, vol. 35, pp. 523-528, apr 2012.

[25] C. Heller, D. Hillis, D. Sadava, G. Orians, and W. Purves, Life: the science of biology. Sinauer Associates, Inc., eigth ed., 2007.

[26] D. Purves, G. Augustine, and D. Fitzpatrick, Neuroscience. Sinauer Associates Inc., 2 ed., 2001.

[27] J. Dargahi and S. Najarian, "Human tactile perception as a standard for artificial tactile sensing- a review," Int J Medical Robotics and Computer Assisted Surgery, vol. 1, pp. 23-35, April 2004.

[28] K. Johnson, "The roles and functions of cutaneous mechanoreceptors," Curr Opin Neurobiol., vol. 11, pp. 455-61, August 2001.

[29] University of Calgary, "Somatosensory system 2." http://www.ucalgary.ca/ pip369/mod7/touch/somato2. [Online; November 14, 2016].

[30] Precision Microdrives, "Vab-02: How do vibration motors work?" http: //www .precisionmicrodrives.com/application-notes-technical-guides/ application-bulletins/vab-02-how-do-vibration-motors-work. [Online; accessed December 10, 2015].

[31] Precision Microdrives, "Ab-004: Understanding erm vibration motor characteristics." http://www.precisionmicrodrives.com/ application-notes-technical-guides/application-bulletins/ ab-004-understanding-erm-characteristics-for-vibration-applications. [Online; accessed December 11, 2015]. 
[32] Precision Microdrives, "Linear resonant actuators- lras." https://www .precisionmicrodrives.com/vibration-motors/ linear-resonant-actuators-lras. [Online; accessed December 11, 2015].

[33] Precision Microdrives, "Driving linear resonance vibration actuators." https://www.precisionmicrodrives.com/application-notes/ ab-003-driving-linear-resonance-vibration-actuators. [Online; accessed December 12, 2015].

[34] Texas Instruments, "Drv2603 haptic drive with auto-resonance detection for linear resonance actuators (lra)." http://www.ti.com/lit/ds/symlink/drv2603. pdf. [Online; accessed November 30, 2016].

[35] J. Melhuish, M. Clark, K. Harding, and R. Williams, "The effects of compression bandage application technique upon measured sub-bandage pressures," Wounds, vol. 17, pp. 243-6, 2005.

[36] PennState Eberly College of Science, "Sensitivity, specificity, positive predictive value, and negative predictive value." https://onlinecourses.science.psu. edu/stat507/node/71. [Online; accessed July 15, 2016].

[37] K. Kaivanto, "Maximization of the sum of sensitivity and specificity as a diagnostic cutpoint criterion," Journal of Clinical Epidemiology, vol. 61, June 2008.

[38] P. Crawford and E. Zimmerman, "Differentiation and diagnosis of tremor," Am Fam Physician, vol. 83, pp. 697-702, March 2011.

[39] E. Rocon and J. Pons, Case study: upper limb tremor suppression through impedance control. Wiley, 2008.

[40] Precision Microdrives, "Dc and brushless motor testing services." https://www.precisionmicrodrives.com/services-capabilities/ dc-brushless-motor-testing-services, 2016. [Online; December 9, 2015].

[41] W. Nanhoe-Mahabier, J. H. Allum, E. P. Pasman, S. Overeem, and B. R. Bloem, "The effects of vibrotactile biofeedback training on trunk sway in Parkinson's disease patients," Parkinsonism and Related Disorders, vol. 18, no. 9, pp. 10171021, 2012.

[42] B.-C. Lee, B. J. Martin, and K. H. Sienko, "The effects of actuator selection on non-volitional postural responses to torso-based vibrotactile stimulation.," Journal of neuroengineering and rehabilitation, vol. 10, p. 21, 2013. 
[43] L. J. F. Janssen, L. L. Verhoeff, C. G. C. Horlings, and J. H. J. Allum, "Directional effects of biofeedback on trunk sway during gait tasks in healthy young subjects," Gait \& $\&$ Posture, vol. 29, no. 4, pp. 575-581, 2009.

[44] A. Tino, M. Carvalho, N. Preto, and K. McConville, "Wireless vibrotactile feedback system for postural response improvement," 2011 33rd Annual International Conference of the IEEE Engineering in Medicine and Biology Society, 2011.

[45] Arduino, "Arduino pin current limitations." http://playground.arduino.cc/ Main/ArduinoPinCurrentLimitations. [Online; accessed on September 7, 2016].

[46] Precision Microdrives, "Product data sheet pico vibe $10 \mathrm{~mm}$ vibration motor - 3mm type, model 310-003." https://www.precisionmicrodrives.com/ product/310-003-10mm-vibration-motor-3mm-type. [Online; accessed Feb 20, 2016].

[47] J. H. Allum, M. G. Carpenter, B. C. Horslen, J. R. Davis, F. Honegger, K.-S. Tang, and P. Kessler, "Improving impaired balance function: Real-time versus carry-over effects of prosthetic feedback," Proceedings of the Annual International Conference of the IEEE Engineering in Medicine and Biology Society, EMBS, pp. 1314-1318, 2011.

[48] E. Kentala, J. Vivas, and C. r. Wall, "Reduction of postural sway by use of a vibrotactile balance prosthesis prototype in subjects with vestibular deficits.," The Annals of otology, rhinology, and laryngology, vol. 112, pp. 404-409, may 2003.

[49] A. U. Alahakone and S. A. Senanayake, "A combination of inertial sensors and vibrotactile feedback for balance improvements in therapeutic applications," 2009 Innovative Technologies in Intelligent Systems and Industrial Applications, CITISIA 2009, pp. 5-10, 2009.

[50] F. Honegger, I. Hillebrandt, N. van den Elzen, Kok-Sing Tang, and J. Allum, "The effect of prosthetic feedback on the strategies and synergies used by vestibular loss subjects to control stance," Journal of NeuroEngineering and Rehabilitation, vol. 10, 2013.

[51] M. Rossi-Izquierdo, A. Ernst, A. Soto-Varela, S. Santos-Pérez, A. FaraldoGarcía, Á. Sesar-Ignacio, and D. Basta, "Vibrotactile neurofeedback balance training in patients with Parkinson's disease: Reducing the number of falls," Gait $\& 3$ Posture, vol. 37, no. 2, pp. 195-200, 2013. 
[52] U.S. Department of Health and Human Services, "Anthropometric reference data for children and adults: United states, 2007-2010." http://www.cdc.gov/nchs/ data/series/sr_11/sr11_252.pdf, October 2012.

[53] R. Simpson and C. Bolton, "An anthropometric survey of 200 r.a.f. and r.n. aircrew and the application of the data to garment size rolls." http://naca. central.cranfield.ac.uk/reports/arc/rm/3612.pdf, 1970.

[54] I. Griffiths, Principles of Biomechanics and Motion Analysis. Lippincott Williams and Wilkins, 2006.

[55] STMicroelectronics, "Tilt measurement using a low-g 3-axis accelerometer." http://www.st.com/content/st_com/en/search.html\#q= Tiltmeasurementusingalow-g3-axisaccelerometer-t=keywords-page=1, June 2014.

[56] M. Pedley, "Tilt sensing using a three-axis accelerometer." https://www .nxp. com/files/sensors/doc/app_note/AN3461.pdf. [Online; accessed December $11,2016]$.

[57] W. Higgins, "A comparison of complementary and kalman filtering," IEEE Trans. Aerospace and Electronic Systems, vol. 11, pp. 321-5, May 1975.

[58] M. Shahdloo, S. Sharifi, and G. Vossoughi, "Precise tilt angle detection using gyro and accelerometer sensor fusion," February 2014.

[59] O. Bastelseiten, "Imu data fusing: Complementary, kalman, and mahony filter." http://www.olliw.eu/2013/imu-data-fusing/\#chapter21, September 2013. [Online; accessed November 11, 2016].

[60] K. H. Sienko, "Perturbation-based detection and prosthetic correction of vestibulopathic gait.," 2007.

[61] C. Wall, L. Oddsson, F. Horak, D. Wrisley, and M. Dozza, "Applications of vibrotactile display of body tilt for rehabilitation," Conference Proceedings. 26th Annual International Conference of the IEEE Engineering in Medicine and Biology Society (IEEE Cat. No.04CH37558), 2004.

[62] F. Faul, E. Erdfelder, A. Lang, and A. Buchner, "G*power 3: A flexible statistical power analysis program for the social, behavioral, and biomedical sciences," Behavior Research Methods, vol. 39, pp. 175-91. 
[63] F. Asseman, A. Bronstein, and M. Gresty, "Effectiveness of a vibro-tactile feedback to cue a stepping response to a balance challenge," 2006 IEEE International Workshop on Haptic Audio Visual Environments and Their Applications (IEEE Cat. No. 06EX1598C), 2006.

[64] B.-C. C. Lee, J. Kim, S. Chen, and K. H. Sienko, "Cell phone based balance trainer," J Neuroeng Rehabil, vol. 9, p. 10, 2012.

[65] K. E. Bechly, W. J. Carender, J. D. Myles, and K. H. Sienko, "Determining the preferred modality for real-time biofeedback during balance training.," Gait 83 posture, vol. 37, pp. 391-396, mar 2013. 